\title{
Multidimensional Coherent Spectroscopy of GaAs Excitons and Quantum Microcavity Polaritons
}

Brian L. Wilmer

Follow this and additional works at: https://researchrepository.wvu.edu/etd

\section{Recommended Citation}

Wilmer, Brian L., "Multidimensional Coherent Spectroscopy of GaAs Excitons and Quantum Microcavity Polaritons" (2016). Graduate Theses, Dissertations, and Problem Reports. 6959.

https://researchrepository.wvu.edu/etd/6959

This Dissertation is protected by copyright and/or related rights. It has been brought to you by the The Research Repository @ WVU with permission from the rights-holder(s). You are free to use this Dissertation in any way that is permitted by the copyright and related rights legislation that applies to your use. For other uses you must obtain permission from the rights-holder(s) directly, unless additional rights are indicated by a Creative Commons license in the record and/ or on the work itself. This Dissertation has been accepted for inclusion in WVU Graduate Theses, Dissertations, and Problem Reports collection by an authorized administrator of The Research Repository @ WVU.

For more information, please contact researchrepository@mail.wvu.edu. 


\title{
Multidimensional Coherent Spectroscopy of
}

\section{GaAs Excitons and Quantum Microcavity Polaritons}

\author{
Brian L. Wilmer
}

\author{
Dissertation submitted to the \\ Eberly College of Arts and Sciences \\ at West Virginia University \\ in partial fulfillment of the requirements \\ for the degree of \\ Doctor of Philosophy \\ in \\ Physics
}

\author{
Alan D. Bristow, Ph.D., Chair \\ Loren D. Anderson, Ph.D. \\ Fabien Goulay, Ph.D. \\ James P. Lewis, Ph.D. \\ Tudor D. Stanescu, Ph.D. \\ Department of Physics and Astronomy \\ Morgantown, West Virginia \\ 2016
}

Keywords: Four-wave Mixing, Multidimensional Coherent Spectroscopy, Two-dimensional Fourier Transform Spectroscopy, Nonlinear Spectroscopy, Excitons, Biexcitons, Microcavity, Exciton-Polaritons, Strain

Copyright 2016 Brian L. Wilmer 


\section{Abstract}

Light-matter interactions associated with excitons and exciton related complexes are explored in bulk GaAs and semiconductor microcavities using multidimensional coherent spectroscopy (MDCS). This approach provides rich spectra determining quantum excitation pathways, structural influences on the excitons, and coherence times.

Polarization, excitation density, and temperature-dependent MDCS is performed on excitons in strained bulk GaAs layers, probing the coherent response for differing amounts of strain. Biaxial tensile strain lifts the degeneracy of heavy-hole and light-hole valence states, leading to an observed splitting of the associated excitons at low temperature. Increasing the strain increases the magnitude of the heavy-/light- hole exciton peak splitting, induces an asymmetry in the offdiagonal interaction coherences, increases the difference in the heavy- and light- hole exciton homogenous linewidths, and increases the inhomogeneous broadening of both exciton species. All results arise from strain-induced variations in the local electronic environment, which is not uniform along the growth direction of the thin layers. For cross-linear polarized excitation, wherein excitonic signals give way to biexcitonic signals, the high-strain sample shows evidence of bound light-, heavy- and mixed- hole biexcitons.

2DCS maps the anticrossing associated with normal mode splitting in a semiconductor microcavity. For a detuning range near zero, it is observed that there are two diagonal features related to the intra-action of exciton-polariton branches and two off-diagonal features related to coherent interaction between the polaritons. At negative detuning, the line shape properties of the diagonal intra-action features are distinguishable and can be associated with cavity-like and exciton-like modes. A biexcitonic companion feature is observed, shifted from the exciton feature by the biexciton binding energy. Closer to zero detuning, all features are enhanced and the diagonal intra-action features become nearly equal in amplitude and linewidth. At positive detuning the exciton-like and cavity-like characteristics return to the diagonal intra-action features. Off-diagonal interaction features exhibit asymmetry in their amplitudes throughout the detuning range. The amplitudes are strongly modulated as the lower polariton branch crosses the bound biexciton energy determined from negatively detuned spectra. 
Dedication

To dedication. 


\section{Acknowledgements}

I would like express gratitude to my adviser, Dr. Alan Bristow. I really could not imagine having worked for anyone else. I also owe much thanks to Dr. Earl Scime, Dr. Leonardo Golubovic, and Dr. Larry Halliburton and the rest of the admissions committee for the opportunity to come to the WVU Physics Department.

I must also thank my comrades who made the time here even more enjoyable, notably Robyn Trappen, James Franek, Dr. Joseph Rowley, Dr. Tess Senty Oliver, Dr. Derek Bas, Dr. Scott Cushing, Dr. Kineshma Mundbodh, Dr. Phil Tabor, and Harley Hart. I was also fortunate to have many great undergraduates to keep me coming in on time during the summers. Of course I must also thank my Family and fiancé Dr. Irais Valencia Jaime for their love and support.

I would like to thank the National Science Foundation (CBET-1233795) and the WV Higher Education Policy Commission (HEPC.dsr.12.29). 


\section{Contents}

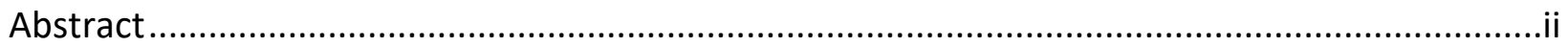

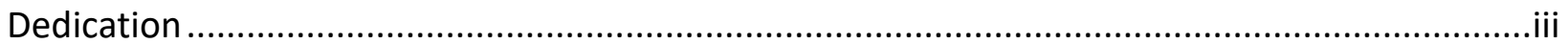

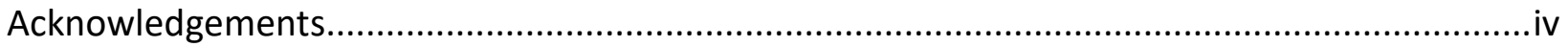

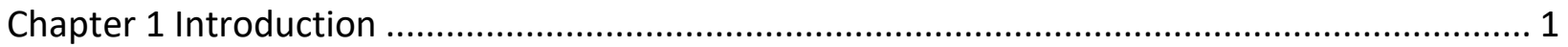

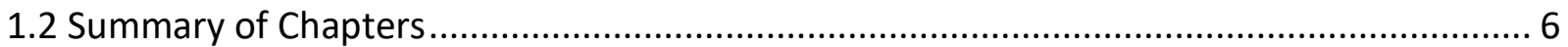

Chapter 2 Theory of FWM and MDCS................................................................................ 7

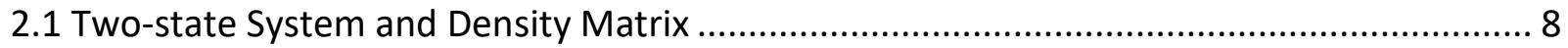

2.2 Extension of the Two-state System and Further Approximations ................................. 10

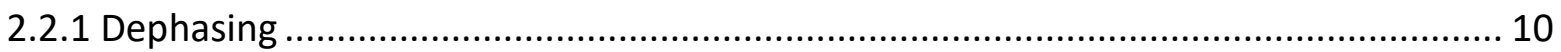

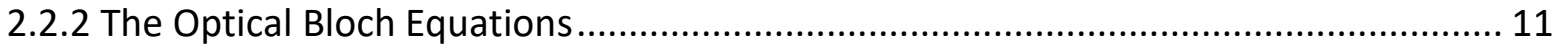

2.2.3 Perturbative Expansion of the Density Matrix ................................................... 12

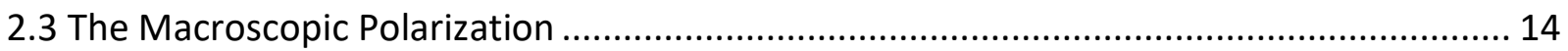

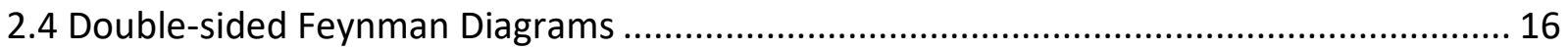

2.5 Two-Dimensional Correlation Spectroscopy ............................................................ 19

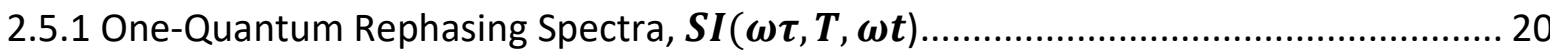

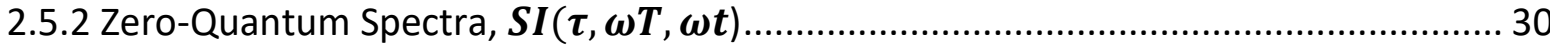

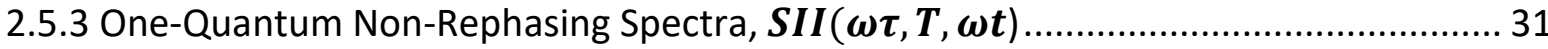

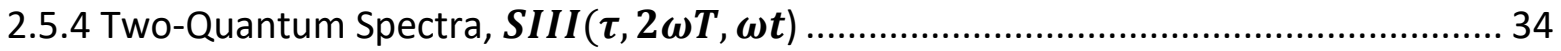

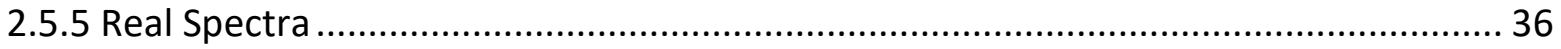

Chapter 3 Experimental and Technical Background Information ......................................... 39

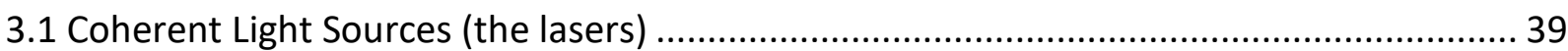

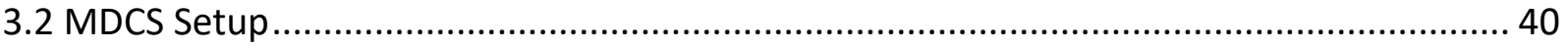

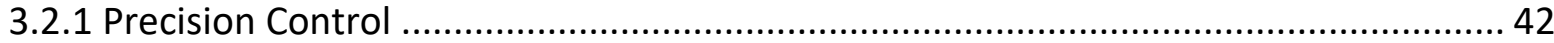




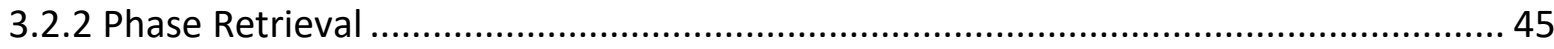

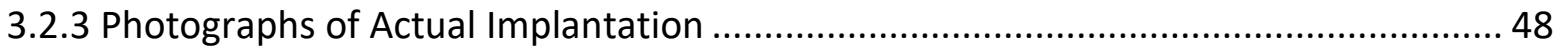

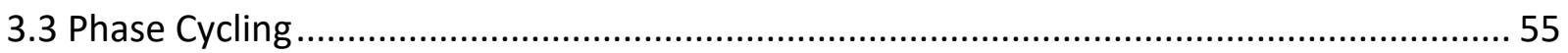

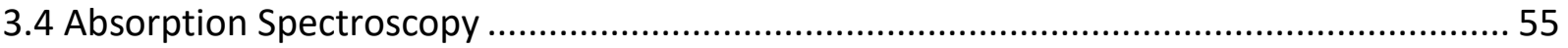

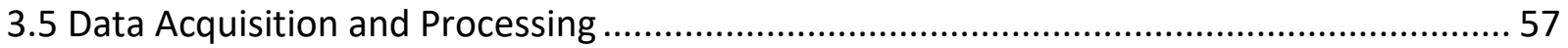

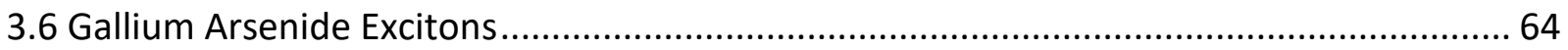

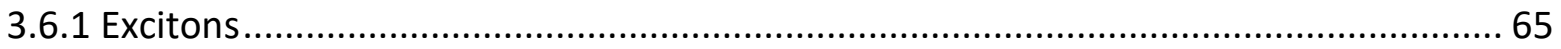

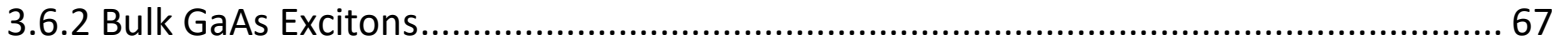

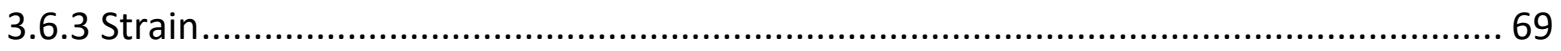

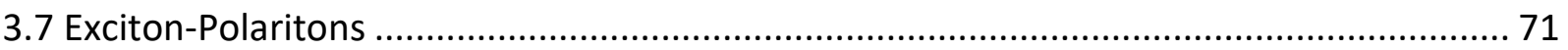

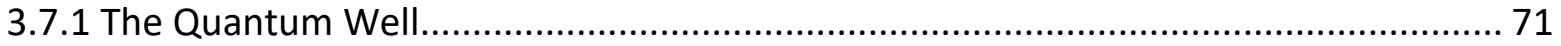

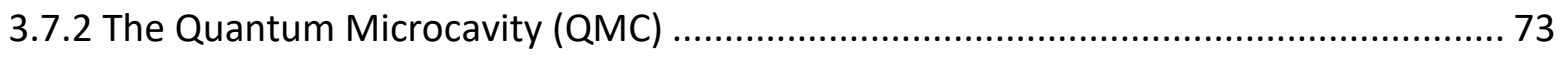

Chapter 4 Bulk Gallium Arsenide Excitons-Results .................................................................... 79

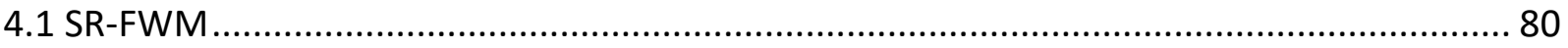

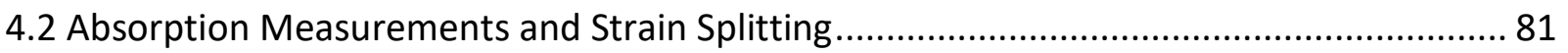

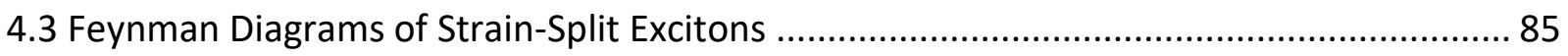

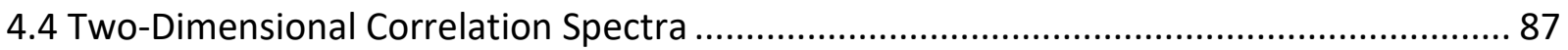

4.5 Power and Temperature Dependences in the High-Strain Sample ................................... 96

4.6 Non-radiative Coherences...................................................................................... 104

4.7 Two-quantum Contributions...................................................................................... 105

4.8 Conclusions from Study of Strained Bulk GaAs Excitons ............................................... 106

Chapter 5 Quantum Microcavity Polaritons-Results .............................................................. 108

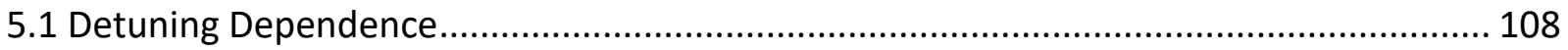

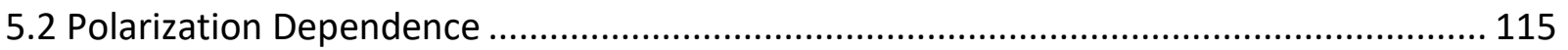


5.3 Two-Quantum Data

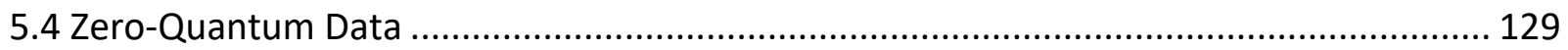

5.5 Conclusions from Study of a Quantum Microcavity ................................................. 130

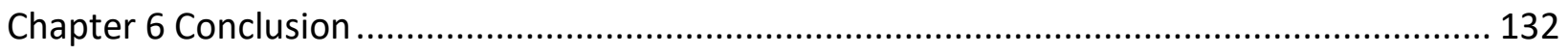

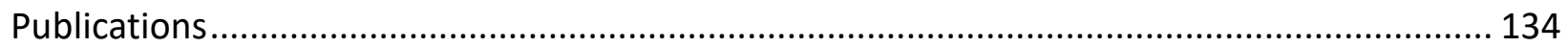

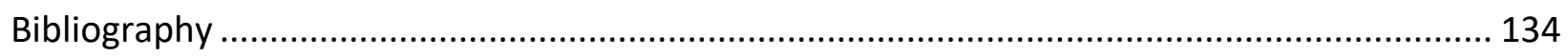

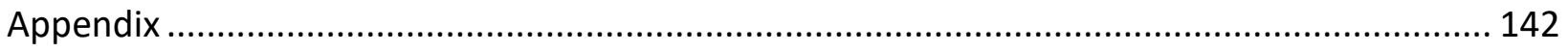

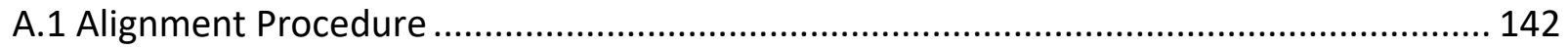

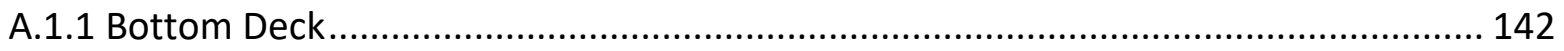

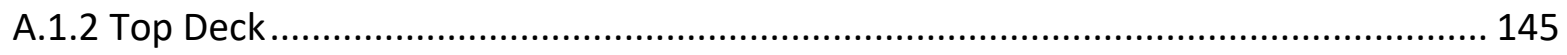

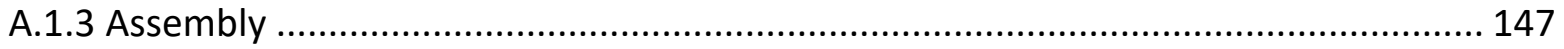

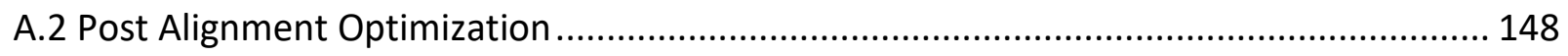

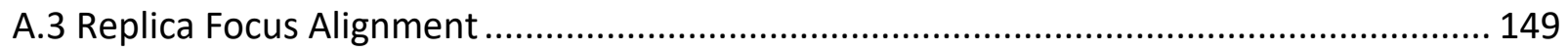

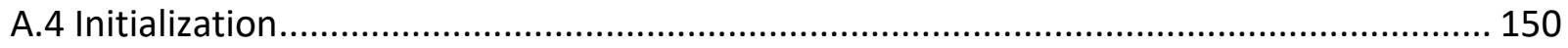

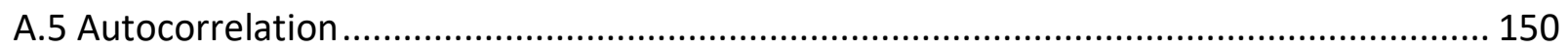

A.6 Calibration of the SPEX 1702, 1 Meter Spectrometer ............................................ 154

A.7 2D Data Acquisition Procedure, with Phase Retrieval .............................................. 156

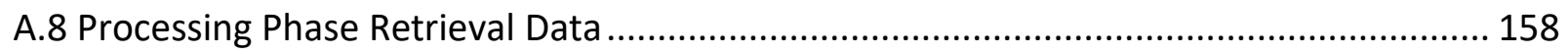

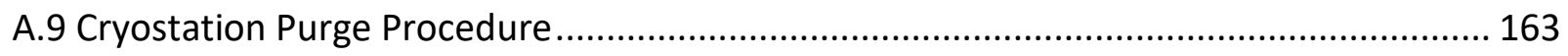

A.10 Prepare Spectra for FFT (Prep2DFiles.m) ......................................................... 164

A.11 FTSA (Fourier Transform Spectral Analysis) Code (FTSA.m) ................................... 167

A.12 Calculate "Camera Phase" (lineout.m) ................................................................ 183

A.13 Linear Absorption and Ideal Resonance Linehsapes code (LinAbsorption2.m) ............ 185

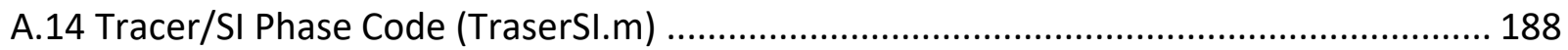


A.15 Peak Fitting Code (Linewidth_coupledfitCleaned.m) ............................................ 192

A.16 Peak Integration Code (integrateregion2.m) ................................................... 198

A.17 Neutral Density Filter Correction Code (ND_Adj.m) ................................................. 208

A.18 Collect Individual Data Files for Large Strain Data Set (aggregator.m) ....................... 211

A.19 Render Various Plots from Strain Data Set (aggplotter.m) ..................................... 213 


\section{Chapter 1 Introduction}

Photonics is the study of light matter interactions and the development of technology that absorbs, modulates or emits light. With the advent of coherent light references, high quality growth techniques, and ever shrinking device sizes, we are in the regime when light, combined with other appropriate materials, is very useful for technological purposes. Solar cells $[1,2]$, new solid-state light references [3-5], sensitive light detectors [6-10], communications networks [11-14]...are some of the technologies that have come to fruition and continue to progress in recent times driven by light-matter interactions. A consistent challenge is to use the short electric or magnetic field pulses to move beyond spectroscopy and coherently control the light-matter interactions. One could then transmit, manipulate, and store information in a welldefined quantum mechanical state just like a traditional computer, except that now one uses quantum states of matter and light. Technology based on these mechanisms would be orders of magnitude superior to their traditional counterparts [15-17]. In this quantum regime, properties such as coherence times will be required and moreover, coherent control could be used to understand and improve chemical reactions, material properties, etc..

MDCS was developed in the 1970's as an extension of nuclear magnetic resonance (NMR) to study coupled resonances. This technique was extended to electronic frequencies which are in the optical frequency range. Studying electronic dynamics means one is studying polarization. Experimentally, one generates polarizations using electric fields typically supplied by lasers and monitors the emitted radiation. The induced polarization is proportional to the applied electric fields. To first order, that induced polarization or oscillation is simply proportional to the product of the electric field and the first order susceptibility which describes the materials response. This linear response describes most of the phenomena we experience: Absorption, luminescence, refraction, reflection, etc. You can also think of this equation parametrically, in that it only requires ONE interaction with the applied field. However, if driven sufficiently hard, nonlinear responses begin to emerge and the polarization can be expanded phenomenologically in a Taylor series. In this work I use MDCS to study the third order response, which is called FWM. In general, studying the nonlinear terms allows for more detailed study of a sample because the response depends on multiple interactions or beams which can be individually varied to probe the 
material. MDCS refers to correlation techniques which have multiple axes of spectral resolution, in particular for this work multiple axes which result from Fourier-transforms. Multiple axes of spectra resolution allow for clearer disambiguation of different types of spectral linewidths which would be convolved in one-dimensional spectra. It also quantifies coupling between states as separable peaks which would also be convolved in one-dimensional spectra. See Chapter 3.6 and references therein.

MDCS is based on and supersedes FWM. Common linear spectroscopies such as absorption and photoluminescence will supply information about which states exist in a system. But since these on require one interaction with an excitation field, they reveal nothing about coherent coupling between states and it is difficult to extract homogeneous and inhomogeneous linewidths as the dominant linewidth is lifetime broadening in these measurements. In semiconductors, many-body interactions are significant and increase with excitation density. So nonlinear spectroscopies are more suited to their study as well as coherent coupling and how it is affected by these interactions. Furthermore, one dimensional spectroscopies, even if nonlinear, make extraction of linewidths and coupling difficult as the data is totally convolved along one frequency domain. The present work was done in two frequency domains, and thus can be called two-dimensional correlation spectroscopy (2DCS), a subset of MDCS. Three frequency domains have also been accomplished [18-20]. In principle, the number of possible domains is only limited by the number of variable time delays between pulses.

At optical frequencies, MDCS has been utilized to study various semiconductor nanostructures [21-27]. This technique retains both time and frequency resolution, is able to unambiguously distinguish a variety of quantum pathways (including those with nonradiative steps) [28], and can separate homogeneous and inhomogeneous broadening [29]. The technique is an extension of two-dimensional spectroscopy developed in NMR [30,31] to study the coherent response of atoms but at much higher, electronic, frequencies first implemented by $\mathrm{Hybl}$ et al $[32,33]$ in 1998. Other energy regimes have been realized as well, such as ultra-violet, infra-red ( 200 meV) studying vibrational modes in molecules [34,35], and various low energy excitations in semiconductors in the terahertz (4-100 meV) such as phonons, electronic interband transitions, and charge transport [36], and excited molecular states and carbon nanotubes in the 
ultra-violet range $(\sim 5,000 \mathrm{meV})[37,38]$. Other specific structures which have been studied include carbon nanotubes [39] and other nanostructures such as quantum dots $[40,41]$ and wells [22,29,42], microcavities [43], Nitrogen vacancies [44], colloidal nanostructures [45,46], atomic vapors [47], and organic photosynthetic light harvesting molecules [48].

At these frequencies in the near infrared ( 1000 meV), implementation of the experiment is much more challenging than the NMR realizations because of the sensitivity of the signal to fluctuations in excitation phase caused by vibrations of the optics on the order of 100's of nanometers. To overcome this barrier, active and passive stabilization has been utilized to achieve path length stability of $<\lambda / 100$ which is on the order of a few nanometers.

To date, two-quantum [49-51], and higher-order [24,52], coherent spectra have shown that many-body interactions dominate the signals, including a contribution from bound biexcitons for excitation with the correct polarization configuration. The experimental setup is described in publications by Bristow et al $[53,54]$.

Gallium Arsenide is a direct bandgap III-IV semiconductor with a zinc-blend crystal structure, with a bandgap in the near infrared $\sim 1.423 \mathrm{eV}$ and a lattice constant of $5.658 \AA$ at room temperature. It has been used to grow a wide range of heterostructures ranging from bulk samples to those with diminishing dimensionality such as quantum wells and quantum dots. Optical spectra of GaAs excitons were first observed in 1961-2 [55-57] and the material has been extensively studied using linear and nonlinear spectroscopy. Excitons are quasi-particles which consist of a bound positive and negative charge. A review of all work relevant to GaAs excitons is beyond the scope of this single thesis. Though in spite of this, all of the physics determining the evolution of excitations of the system are not known and so it continues to be a semiconductor used to study light-matter interactions. Four wave mixing of strained GaAs was first done in 19923 using one-dimensional spectroscopic techniques (pump-probe and time resolved measurements) $[58,59]$ but these measurements suffer from the drawbacks of one-dimensional techniques, as discussed in chapter 2 . The MDCS measurements in this work provide much new insight into the behavior of excitons in bulk and strained GaAs which contribute more generally 
to the body of knowledge regarding how correlated quasi-particles behave in semiconductor crystals.

To understand the implications of strain on quantum applications the entire response, including coherent properties, must be understood. Coherent nonlinear optical techniques, such MDCS, provide a more comprehensive picture of the excitonic response compared to linear optical techniques. MDCS may be exploited to gain further insight into the influence of strain on the coherent optical response of semiconductors. For instance, the ability to independently measure the levels of homogeneous and inhomogeneous broadening [29,60-64] may yield insight into varying local electronic environments tied to partial strain relaxation in thin film systems. MDCS has also provided a wealth of information about many-body interactions in quantum well systems through the ability to isolate a range of quantum pathways from the excitons, their coupling to one another, and biexcitons [19,21,25,26,28,49,50,65-68]. The study of such effects in a bulk semiconductor system, in which the splitting between the exciton resonances is caused exclusively by strain, would provide insight into the influence of strain on $\mathrm{MBI}$ and the coherent response of the excitons. [69]

Strain engineering provides a powerful method to tailor the electronic and optical properties of semiconductors, a feature that has been utilized in a variety of applications in semiconductor opto-electronics. For instance, the controlled use of strain has led to lowthreshold lasers using strained quantum wells or quantum dot systems, [70-73] and fast fieldeffect transistors based on the SiGe/Si system. [74] Manipulation of strain may also lead to the development of polarization-entangled photon pairs through control of the relative size of the exciton and biexciton binding energies in quantum dot systems, [75] and provides a means to dynamically manipulate the spin-orbit interaction for possible spin-sensitive electronic devices [76,77]. For such applications, an accurate characterization of the influence of strain on the local and global optoelectronic properties in the semiconductor heterostructure is essential. Existing studies of strain in semiconductor systems have largely focused on its influence on the energetic locations of the heavy-hole and light-hole exciton resonances, detected using optical techniques such as photoluminescence, reflectivity and Raman scattering [78-84]. 
In this work, MDCS is used to study bulk excitons in two GaAs layers with different thicknesses, such that the two samples exhibit different amounts of strain, resulting from being attached to sapphire disks and cooled. Comparison of rephasing 2DCS spectra with collinear and cross-linear polarization configurations allows for the observation of the line shapes, center positions, homogeneous and inhomogeneous linewidths, and the coupling of excitons and biexcitons. In addition to increasing the separation between exciton species, these experiments show that increasing the amount of strain in the bulk semiconductor modifies the relative strengths of the off-diagonal exciton features, altering the spectrum from a coherent response typically seen in atomic vapors to one seen in quantum wells. A larger degree of inhomogeneous broadening is also observed in the sample with larger strain, attributed to variations in the local electronic environment that are likely tied to strain gradients along the growth direction of the heterostructure. Significant bound biexciton signatures are visible for cross-linear polarized excitation, due to the presence of few-body interactions. Overall, the results suggest that 2DCS and the coherent response of GaAs excitons is highly sensitive to strain.

The normal-mode coupling in a quantum microcavity between the photonic cavity mode $\left(E_{C}\right)$ and the exciton resonance $\left(E_{X}\right)$ enhances both the linear and nonlinear optical interactions [85] and was observed by Weisbuch et al. in 1992 [86]. TFWM confirms that strong exciton-cavity interactions modify the temporal behavior of the coherent response and manybody Coulomb correlations determine the exact dynamics [87], which affects dissipation [88-90] and coherent control [91,92]. Biexciton-polaritons also contribute to the overall emitted signal, even though the biexciton binding energy is only slightly altered by the cavity $[93,94]$. Semiconductor microcavities supporting exciton-polaritons $[95,96]$ are used in optoelectronic applications $[3,97,98]$ and provide a platform for exploring exotic coherent physical phenomena [99-104], including but not limited to Bose-Einstein condensate behavior owing to the lighter effect mass resulting from the photonic polariton component [88,105-109].

Despite extensive previous study, all of the dynamics are not understood. In particular, the complicated interactions between excitons, polaritons, and their upper hierarchies of bound states are not completely understood. Two- and three-quantum MDCS measurements were conducted by [52] at a single detuning as well as coherence and control of excitonic qubits in 
microcavity pillars [110]. However, one-quantum manifold scans, detuning mappings, and systematic polarization dependences have not been performed. This this work as well as $[103,111,112]$ illustrated the rich variety of states and hierarchies of bound and correlated states and their significant contribution to the coherent response. The MDCS work underscored its utility in understanding this system. The work in this dissertation thoroughly studies the onequantum response with high-resolution detuning dependences as well as polarization control. Similar zero- and two-quantum studies are ongoing. See Chapter 3.5 and references therein.

Generally, the work in this dissertation provides insight into the nature of states that exist in real systems and evolve in environments with other excited states as well. Using MDCS to study nonlinear emission from samples will provide the rich spectra necessary for and capable of elucidating the rich many-body and few-body physics dominating the nonlinear response, including coherence times, etc.

\subsection{Summary of Chapters}

Chapter 2 communicates theory of four-wave mixing and multidimensional correlation spectroscopy. I will proceed with developing the formalism further specifically in the context of two-dimensional correlation spectroscopy (2DCS), a subset of MDCS. A series of examples with simulated spectra of model systems exhibiting hallmark properties will demonstrate the power of the technique. The specific scan types and their utility is stated at the end of the chapter. Chapter 3 starts with information regarding the coherent light sources used, the MONSTR which is a tool for controlling the laser fields, information regarding retrieving the global phase of the signal field, photorgraphs of the experimental seteup. Absportion spectroscopy is discussed as well as data acquisition and processing of a typical 2DCS scan. Latter sections report necessary background information for a working understanding of the samples. This information includes the definition of excitons, the effects of strain on them in GaAs, the definition of quantum wells, their placement in a microcavity, and the resulting exciton-polaritons. Chapters 4 and 5 report the results of optical absorption, SR-FWM, and MDCS on strained bulk GaAs and a quantum microcavity, respectively. 


\section{Chapter 2 Theory of FWM and MDCS}

In this work we study radiation emitted by a sample in order to learn about the excited, charged particles which created it. The particles are created and put in motion by applied electric fields from a pulsed laser. The following theory in this chapter relating applied electric fields and the resulting induced polarization of charge can be found in most nonlinear optics textbooks [113-115].

The classical, phenomenological relation relating the induced (macroscopic) polarization density, which is the dipole moment per unit volume, to the applied electric field is a Taylor series:

$$
\boldsymbol{P}(t)=\epsilon_{o} \chi^{(1)} \boldsymbol{E}(\boldsymbol{t})+\epsilon_{o} \chi^{(2)} \boldsymbol{E}^{\mathbf{2}}(\boldsymbol{t})+\epsilon_{o} \chi^{(3)} \boldsymbol{E}^{\mathbf{3}}(\boldsymbol{t})+\epsilon_{o} \chi^{(4)} \boldsymbol{E}^{\mathbf{4}}(\boldsymbol{t})+\cdots
$$

Where $\boldsymbol{E}$ is the applied electric field, $\epsilon_{o}$ is the permittivity of free space, and $\chi^{(n)}$ is the nth order susceptibility characterizing the material's response.

The first term is responsible for all linear phenomena such as absorption, luminescence, (linear) index of refraction, etc. It requires only a single applied electric field, to say excite one transition, which then generates the radiating polarization when it relaxes back to the ground state for example. The second term describes phenomena requiring two interactions with a driving field, such as sum frequency generation where two photons generate a polarization which radiates at the sum of the energies of the input photons. Second-order effects only occur in crystals lacking inversion symmetry and is therefore zero in many materials. The third-order response is responsible for the radiation studied in this work, the so-called four-wave mixing signal. The name results from the four waves which interact-the three exciting beams and their resulting signal. Similarly, the fifth order response is six-wave mixing and so forth. The thirdorder response does not require any special symmetries or lack thereof, is nonzero in all crystals, and frequently a material's first nonzero nonlinearity. It is also responsible for Kerr lensing, which is an intensity dependent index of refraction exploited in ultrafast pulsed lasers, described further in section 3.1 . 
Generally, the problem with exciting the sample with one dose of radiation and monitoring the response is that many different response mechanisms' emission will be spatially overlapped, including through-put of the laser beam. Nonlinear spectroscopy allows for isolating the response from each term in the nonlinear expansion and selective coherent control of the material's response. Nonlinear optics and spectroscopy allow for examining a system's response to interaction with each field with great sensitivity.

As we will be studying quantum mechanical systems, a semi-classical theory is at least necessary and will be developed in the following sections.

\subsection{Two-state System and Density Matrix}

Density matrix formalism will be used to describe the systems in this work $[114,116]$. It is well suited for describing statistical ensembles of systems, such as those encountered experimentally. To understand the basic mechanics of how this formalism works, let us consider a two state system with a single ground $|1\rangle$ and excited state $|2\rangle$, having energies $E_{1}$ and $E_{2}$, respectively. The two allowed transitions for this system are absorption or emission of energy at $E_{12}=E_{1}-E_{2}$. Much intuition can be gained from the two state system, and the formalism can be straight forwardly extended to more complex systems.

The time-dependent density matrix is defined as:

$$
\rho(t) \equiv \sum_{i} p_{i}\left|\psi_{i}(t)\right\rangle\left\langle\psi_{i}(t)\right|
$$

Where $p_{i}$ is the probability of the $i^{\text {th }}$ particle, being in the state $\left|\psi_{i}(t)\right\rangle$, which itself may consist of a superposition of the system's eigenstates $|n\rangle$, with time dependent coefficient $c_{n}(t)$, $\left|\psi_{i}(t)\right\rangle=\sum_{n} c_{n}^{i}(t)|n\rangle$. The complex conjugate of which is $\left\langle\psi_{i}\right|=\sum_{n} c_{n}^{* i}(t)\langle n|$. The system is normalized $\sum_{i} p_{i}=1$. Expectation values can be calculated as $\langle X\rangle=\operatorname{Tr}(X \rho)$.

The density matrix is then

$$
\rho(t)=\sum_{i} p_{i} \sum_{n, m} c_{n}^{i}(t) c_{m}^{* i}(t)|n\rangle\langle m|
$$


Performing the sum over $i$ averages the coefficients $c(t)$, over the statistical ensemble, making density matrix

$$
\rho(t)=\left(\begin{array}{ll}
p_{11}(t) & p_{12}(t) \\
p_{21}(t) & p_{22}(t)
\end{array}\right)
$$

where $\rho_{n, m}(t)=\overline{c_{n}(t) c_{m}^{*}(t)}$, where the bar indicates ensemble averaged values. The diagonal entries $(n=m)$ represent probabilities of being in one of the eigenstates, and are referred to as populations. The off diagonal elements $(n \neq m)$ represent the probabilities of being in coherent superpositions of eigenstates, and are referred to as coherences.

The time evolution in the Schrödinger picture is given by:

$$
\frac{d}{d t} \rho=-\frac{i}{\hbar}[H, \rho]
$$

If the system is not under the influence of any external forces, and no population relaxation, then the Hamiltonian is

$$
H=\left(\begin{array}{cc}
E_{1} & 0 \\
0 & E_{2}
\end{array}\right)
$$

The equations of motion are:

$$
\begin{gathered}
\frac{d}{d t} \rho_{11}=\frac{d}{d t} \rho_{22}=0 \\
\frac{d}{d t} \rho_{12}=-\frac{i}{\hbar}\left(E_{1}-E_{2}\right) \rho_{12} \\
\frac{d}{d t} \rho_{21}=\frac{i}{\hbar}\left(E_{1}-E_{2}\right) \rho_{12}
\end{gathered}
$$

Which can be readily solved,

$$
\begin{gathered}
\rho_{11}(t)=\rho_{11}(0) \\
\rho_{22}(t)=\rho_{22}(0) \\
\rho_{12}(t)=e^{-\frac{i}{\hbar}\left(E_{1}-E_{2}\right) t} \rho_{12}(0) \\
\rho_{21}(t)=e^{\frac{i}{\hbar}\left(E_{1}-E_{2}\right) t} \rho_{21}(0)
\end{gathered}
$$


So without any dissipative or external forces, the populations will be constant in time, and the coherences will oscillate forever, at the frequency equal to the difference of the eigenenergies $E_{2}-E_{1}$.

To demonstrate the utility of this notation, even with this simplified system, we can calculate the polarization of the system, which is the expectation value of the dipole moment operator, implicitly assuming that the diagonal entries are zero because states $|1\rangle$ and $|2\rangle$ are assumed to have definite parity (even):

$$
\mu=\left(\begin{array}{cc}
0 & \mu_{12} \\
\mu_{12}^{*} & 0
\end{array}\right)
$$

We find that

$$
P(t)=\langle\mu\rangle=\operatorname{Tr}(\mu \rho)=\mu_{12} \rho_{21}+\mu_{12}^{*} \rho_{12}
$$

Since polarization is responsible for emitting the radiation field, it can be seen that the coherences are responsible for the induced polarization and to understand the system's behavior is to understand how the density matrix evolves.

\subsection{Extension of the Two-state System and Further Approximations}

We will now extend the formalism to a more useful result by applying perturbation theory, phase matching, and so called rotating wave approximation, and loss mechanisms such as relaxation and dephasing. We will then use an easier method to reach the results directly with double-sided Feynman diagrams, with this more formal groundwork established.

\subsubsection{Dephasing}

One of the most important phenomena nonlinear spectroscopy is sensitive to is coherence, which is destroyed by dephasing. The off diagonal elements will decay (dephase) in time, which are themselves coupled to the relaxation rates of the diagonal elements. This dephasing is a manifestation of all microscopic mechanisms that lead to loss of coherence in the system and are generally are denoted as many body interactions (MBEs) because in a real sample there are many excited charge carriers interacting. Dephasing can be added phenomenologically to the equations of motion [114]: 


$$
\frac{d}{d t} \rho_{n m}=-\frac{i}{\hbar}[H, \rho]_{n m}-\gamma_{n m}\left(\rho_{n m}-\rho_{n m}^{e q}\right)
$$

Where $\gamma_{n m}=\frac{1}{2}\left(\Gamma_{n}+\Gamma_{m}\right)+\gamma_{n m}^{\text {pure }}$ is the dephasing rate of the $\rho_{n m}$ coherence, $\Gamma_{n}$ is the

relaxation rate of the population in state $n$, and $\gamma_{n m}^{\text {pure }}$ is the so-called pure (homogeneous) dephasing rate and is 0 for $m=n . \rho_{n m}^{e q}$ is the equilibrium value and is assumed to be zero for $m \neq n$ because in thermal equilibrium the excited states may have a nonzero value but thermal excitation is assumed to be an incoherent process and thus cannot create coherent superpositions. In writing the decay of the populations we have assumed that relaxations are radiative. Therefore, a photon will be preferentially emitted rather than non-radiatively transition to a lower population as the populations are energetically too close and any such population transfer would have to be done with non-radiative processes such as phonons. It is worth pointing out that dephasing cannot be described in the wavefunction formalism.

\subsubsection{The Optical Bloch Equations}

Adding more detail to the earlier example of a two state system under optical excitation. The light-matter interaction Hamiltonian is again $H^{\prime}=-\mu \cdot E(t)$ so that the total Hamiltonian is:

$$
H=H_{o}+H^{\prime}=\left(\begin{array}{cc}
E_{1} & 0 \\
0 & E_{2}
\end{array}\right)+\left(\begin{array}{cc}
0 & -\mu_{12} E(t) \\
-\mu_{12} E(t) & 0
\end{array}\right)
$$

$\mu_{12}$ is the transition dipole between $|1\rangle$ and $|2\rangle$. The leads to the equations of state (with dephasing added):

$$
\begin{gathered}
\frac{d}{d} \rho_{11}=\frac{i}{\hbar} \mu_{12} E(t)\left(\rho_{12}-\rho_{21}\right)-\gamma_{1}\left(\rho_{11}-\rho_{11}^{e q}\right) \\
\frac{d}{d} \rho_{22}=-\frac{i}{\hbar} \mu_{12} E(t)\left(\rho_{12}-\rho_{21}\right)-\gamma_{2}\left(\rho_{22}-\rho_{22}^{e q}\right) \\
\frac{d}{d} \rho_{12}=-\frac{i}{\hbar} \mu_{12} E(t)\left(\rho_{22}-\rho_{11}\right)-\frac{i}{\hbar} \omega_{12} \rho_{12}-\gamma_{12} \rho_{12} \\
\frac{d}{d} \rho_{21}=\frac{i}{\hbar} \mu_{12} E(t)\left(\rho_{22}-\rho_{11}\right)+\frac{i}{\hbar} \omega_{12} \rho_{21}-\gamma_{21} \rho_{21}
\end{gathered}
$$


Where $\omega_{12}=\left|E_{1}-E_{2}\right| / \hbar$. These are the so called Optical Bloch Equations (OBEs) to describe light-matter interactions in two-state systems under the influence of classic fields. The OBEs can be extended further to create the Semiconductor Bloch Equations (SBEs) which include the continuum of states found in solids, and in particular direct band gap semiconductors. the following sections we will apply time-dependent perturbation theory to approximate the systems third-order response.

\subsubsection{Perturbative Expansion of the Density Matrix}

This is also sometimes referred to as the Mukamelian approach, in reference to Shaul Mukamel who has made considerable theoretical contributions to the field [116].The OBE equations can be solved perturbatively by expanding in power of $\rho$, substituting back into the $\mathrm{OBE}$, and collecting powers. However, this is tedious. Another method, sometimes called the Mukamelian approach, is solve the problem in the interaction picture without dephasing and adds the dephasing terms in again phenomenologically to the solution. This is admittedly a shortcoming of the current state of theory, ever progressing, in this relatively new field applying MDCS to electronic systems. It further underscores the necessity to study model systems to understand how various known and unknown physical phenomena manifest.

In the interaction picture, the wavefunctions evolve according to the Schrödinger picture under the influence of the perturbing Hamiltonian $H^{\prime}$, and the operators evolve according to the Heisenberg picture, carrying the time dependence of the unperturbed Hamiltonian $H_{0}$. In the interaction picture, the density matrix (or any other operator) is transformed as:

$$
\rho_{I}(\boldsymbol{r}, t)=U_{0}^{\dagger}\left(t, t_{0}\right) \rho_{S c h o ̈ d}(\boldsymbol{r}, t) U_{0}\left(t, t_{0}\right)
$$

With the unitary time evolution operator (depending only on the unperturbed Hamiltonian) defined as $U_{0}\left(t, t_{0}\right)=e^{i \frac{H_{0}}{\hbar}\left(t-t_{0}\right)}$. Similarly, for the perturbing Hamiltonian,

$$
H_{I}^{\prime}(\boldsymbol{r}, t)=-U_{0}^{\dagger}\left(t, t_{0}\right) \mu E(\boldsymbol{r}, t) U_{0}\left(t, t_{0}\right)
$$

Where I have now explicitly included the wavevector dependence of the driving field with the spatial variable $\mathbf{r}$ which will inevitably drive an important spatial dependence in the density matrix. Using the Liouville-von Neumann equation of motion: 


$$
\frac{d}{d t} \rho_{I}(\boldsymbol{r}, t)=-\frac{i}{\hbar}\left[H_{I}^{\prime}(\boldsymbol{r}, t), \rho_{I}(\boldsymbol{r}, t)\right]
$$

Expand the density matrix in terms of a small parameter $\beta$ :

$$
\rho_{I}(\boldsymbol{r}, t)=\rho_{I}^{(0)}(\boldsymbol{r}, t)+\beta \rho_{I}^{(1)}(\boldsymbol{r}, t)+\beta^{2} \rho_{I}^{(2)}(\boldsymbol{r}, t)+\beta^{3} \rho_{I}^{(3)}(\boldsymbol{r}, t)+\cdots
$$

And insert it into the equation of motion. Then collect same powers of $\beta$ and integrate to solve the equation of motion. The solution order is:

$$
\begin{aligned}
\rho_{I}\left(\boldsymbol{r}, t^{\prime}\right)=\rho_{I} & \left(\boldsymbol{r}_{\mathbf{0}}, t_{0}\right) \\
& +\sum_{N=1}^{\infty}\left(-\frac{i}{\hbar}\right)^{N} \int_{t_{0}}^{t^{\prime}} d t_{N} \int_{t_{0}}^{t_{N}} d t_{N-1} \cdots \int_{t_{0}}^{t_{2}} d t_{1} \\
& \times\left[H_{I}^{\prime}\left(\boldsymbol{r}, t_{N}\right),\left[H_{I}^{\prime}\left(\boldsymbol{r}, t_{N-1}\right), \cdots\left[H_{I}^{\prime}\left(\boldsymbol{r}, t_{1}\right), \rho_{I}\left(\boldsymbol{r}_{0}, t_{0}\right)\right] \cdots\right]\right]
\end{aligned}
$$

Using the definition of the interaction density matrix to switch back over to the Schrödinger picture:

$$
\begin{aligned}
& \rho\left(\boldsymbol{r}, t^{\prime}\right) \\
& =\rho\left(\boldsymbol{r}_{0}, t_{0}\right) \\
& +\sum_{N=1}^{\infty}\left(-\frac{i}{\hbar}\right)^{N} \int_{t_{0}}^{t^{\prime}} d t_{N} \int_{t_{0}}^{t_{N}} d t_{N-1} \cdots \int_{t_{0}}^{t_{2}} d t_{1} \\
& \times U_{0}\left(t^{\prime}, t_{0}\right)\left[H_{I}^{\prime}\left(\boldsymbol{r}, t_{N}\right),\left[H_{I}^{\prime}\left(\boldsymbol{r}, t_{N-1}\right), \cdots\left[H_{I}^{\prime}\left(\boldsymbol{r}, t_{1}\right), \rho_{I}\left(\boldsymbol{r}_{0}, t_{0}\right)\right] \cdots\right]\right] U_{0}^{\dagger}\left(t^{\prime}, t_{0}\right)
\end{aligned}
$$

Substituting in for the interaction Hamiltonian and looking at just the $\mathrm{N}^{\text {th }}$ order perturbative terms and assuming that $\rho_{I}\left(\boldsymbol{r}_{0}, t_{0}\right)$ is a constant which will not evolve in time, let $t_{o} \rightarrow-\infty$ :

$$
\begin{aligned}
& \rho^{(N)}\left(\boldsymbol{r}, t^{\prime}\right) \\
& =\sum_{N=1}^{\infty}\left(-\frac{i}{\hbar}\right)^{N} \int_{-\infty}^{t^{\prime}} d t_{N} \int_{-\infty}^{t_{N}} d t_{N-1} \cdots \int_{-\infty}^{t_{2}} d t_{1} E\left(\boldsymbol{r}_{N}, t_{N}\right) E\left(\boldsymbol{r}_{N-1}, t_{N-1}\right) \cdots E\left(\boldsymbol{r}_{1}, t_{1}\right) \\
& \times U_{0}\left(t^{\prime}, t_{0}\right)\left[\mu_{I}\left(t_{N}\right),\left[\mu_{I}\left(t_{N-1}\right), \cdots\left[\mu_{I}\left(t_{1}\right), \rho_{I}\left(\boldsymbol{r}_{0},-\infty\right)\right] \cdots\right]\right] U_{0}^{\dagger}\left(t^{\prime}, t_{0}\right)
\end{aligned}
$$

where $\mu_{I}=U_{0}^{\dagger}\left(t^{\prime}, t_{0}\right) \mu U_{0}\left(t^{\prime}, t_{0}\right)$. 


\subsection{The Macroscopic Polarization}

We are now in a position to semi-classically describe the polarization induced by the applied electric fields which will then emit the signal radiation. The $N^{\text {th }}$ order macroscopic polarization density is then the expectation value of the dipole moment:

$$
\begin{gathered}
P^{(N)}\left(\boldsymbol{r}, t^{\prime}\right)=\left\langle\mu \rho^{(N)}\left(\boldsymbol{r}, t^{\prime}\right)\right\rangle=\operatorname{Tr}\left(\mu \rho^{(N)}\left(\boldsymbol{r}, t^{\prime}\right)\right) \\
P^{(N)}\left(\boldsymbol{r}, t^{\prime}\right)=\left(-\frac{i}{\hbar}\right)^{N} \int_{-\infty}^{t \prime} d t_{N} \int_{-\infty}^{t_{N}} d t_{N-1} \cdots \int_{-\infty}^{t_{2}} d t_{1} E\left(\boldsymbol{r}, t_{N}\right) E\left(\boldsymbol{r}, t_{N-1}\right) \cdots E\left(\boldsymbol{r}, t_{1}\right) \\
\times\left\langle\mu_{I}\left(t^{\prime}\right)\left[\mu_{I}\left(t_{N}\right),\left[\mu_{I}\left(t_{N-1}\right), \cdots\left[\mu_{I}\left(t_{1}\right) \rho^{(0)}(-\infty)\right] \cdots\right]\right]\right\rangle
\end{gathered}
$$

Let us now limit ourselves to the third-order $(N=3, F W M)$ contribution and label the electric fields as $E_{A}\left(\boldsymbol{r}, t_{A}-t_{A}{ }^{\prime}\right), E_{B}\left(\boldsymbol{r}, t_{B}-t_{B}{ }^{\prime}\right)$, and $E_{C}\left(\boldsymbol{r}, t_{C}-t_{C}{ }^{\prime}\right)$, where $t_{i}$ is the arrival time of the $i^{\text {th }}$ electric field and $t_{i}^{\prime}$ is the integration variable. The fields are laser pulses, each approximated as an enveloped plane wave, generically as:

$$
\boldsymbol{E}(\boldsymbol{r}, t)=\hat{e}\left[\mathcal{E}^{+}(t) e^{i(\boldsymbol{k} \cdot \boldsymbol{r}-\omega t)}+\mathcal{E}^{-}(t) e^{-i(\boldsymbol{k} \cdot \boldsymbol{r}-\omega t)}\right]
$$

With polarization $\hat{e}$, frequency $\omega$, wavevector $\boldsymbol{k}$, and temporal envelopes $\mathcal{E}^{+}$and $\mathcal{E}^{-}$for the positive (unconjugated) and negative (conjugated) frequency components, respectively. The polarization is then $[33,49,113,116,117]$ :

$$
\begin{aligned}
P^{(3)}\left(\boldsymbol{r}, t^{\prime}\right)= & \int_{0}^{\infty} d t_{C}{ }^{\prime} \int_{0}^{\infty} d t_{B}{ }^{\prime} \int_{0}^{\infty} d t_{A}{ }^{\prime} R^{(3)}\left(t^{\prime}, t_{A}, t_{B}, t_{C}\right) \\
& \times E_{C}\left(\boldsymbol{r}, t_{C}-t_{C}{ }^{\prime}\right) E_{B}\left(\boldsymbol{r}, t_{B}-t_{B}{ }^{\prime}\right) E_{A}\left(\boldsymbol{r}, t_{A}-t_{A}{ }^{\prime}\right)
\end{aligned}
$$

With the third-order nonlinear response function defined as (and with interaction picture "I" suppressed):

$$
\begin{aligned}
R^{(3)}\left(t^{\prime}, t_{C}, t_{B},\right. & \left.t_{A}\right) \\
= & \left(-\frac{i}{\hbar}\right)^{3}\left\langle\mu ( t ^ { \prime } ) \left[\mu_{C}\left(t_{C}-t_{C}^{\prime}\right)\right.\right. \\
\cdot & \left.\hat{e}_{C},\left[\mu_{B}\left(t_{B}-t_{B}{ }^{\prime}\right) \cdot \hat{e}_{B},\left[\mu_{A}\left(t_{A}-t_{A}{ }^{\prime}\right) \cdot \hat{e}_{A} \rho^{(0)}(-\infty)\right]\right]\right\rangle
\end{aligned}
$$


Another important, and convenient, assumption is that our exciting pulses are extremely short, specifically their temporal envelope, compared with the time scales of the phenomena under study. This is indeed true, as our coherence times are on the order of several picoseconds and our pulses are $100 \mathrm{fs}$ (a one to two order of magnitude difference). Therefore, we will assume their amplitudes are functionally Dirac delta functions at the arrival time of each pulse and evaluate the integrals. The polarization can then be written as [49]:

$$
\begin{aligned}
P^{(3)}\left(\boldsymbol{r}, t^{\prime}\right)= & R^{(3)}\left(t^{\prime}, t_{C}, t_{B}, t_{A}\right) \mathcal{E}_{C}^{ \pm} \mathcal{E}_{B}^{ \pm} \mathcal{E}_{A}^{ \pm} e^{i\left( \pm \boldsymbol{k}_{A} \pm \boldsymbol{k}_{B} \pm \boldsymbol{k}_{C}\right) \cdot \boldsymbol{r}} e^{-i\left( \pm \omega_{C} \pm \omega_{B} \pm \omega_{A}\right) t^{\prime}} \\
& \times e^{i\left( \pm \omega_{C} \pm \omega_{B} \pm \omega_{A}\right) t_{C}} e^{i\left( \pm \omega_{B} \pm \omega_{A}\right) t_{B}} e^{ \pm i\left(\omega_{A}\right) t_{A}}
\end{aligned}
$$

This polarization could now be inserted into Maxwell's equation as a reference term to generate the radiating polarization, a four-wave mixing signal. The polarization contains all possible permutations of the three applied fields interacting with the sample. Each permutation will emit a signal in the phase-matched direction $\boldsymbol{k}_{s}= \pm \boldsymbol{k}_{A} \pm \boldsymbol{k}_{B} \pm \boldsymbol{k}_{C}$. However since $\boldsymbol{k}_{s}=$ $-\boldsymbol{k}_{S}$, they are not all unique, and there are four permutations that are: $\boldsymbol{k}_{S, I}=-\boldsymbol{k}_{A}+\boldsymbol{k}_{B}+\boldsymbol{k}_{C}$, $\boldsymbol{k}_{S, I I}=+\boldsymbol{k}_{A}-\boldsymbol{k}_{B}+\boldsymbol{k}_{C}, \quad \boldsymbol{k}_{S, I I I}=+\boldsymbol{k}_{A}+\boldsymbol{k}_{B}-\boldsymbol{k}_{C}$, and $\boldsymbol{k}_{S, I V}=+\boldsymbol{k}_{A}+\boldsymbol{k}_{B}+\boldsymbol{k}_{C}$, where the ordering of the letters communicates the time ordering of the pulses (first, second, third...from left to right). Each FWM signal resulting from the different phase matching conditions results from different processes inside the sample. Also note that this is an advantage over linear spectroscopic techniques which are not sensitive to phase matching. This allows one to study only the FWM signal and different processes contributing to it by looking in specific directions.

The latter phase matching condition is not used in this work for three reasons. In the rotating wave approximation where only slowly varying coherences with respect to the laser's center frequency are considered, it is a fast evolving $(3 \omega)$ coherence. Second, phase matching eliminates it because, the apparatus is currently built to accept light emitted in a single particular spatial direction $\left(-\boldsymbol{k}_{A}\right)$. Third, and more pragmatically, the $\boldsymbol{k}_{\text {sig,IV }}$ direction overlaps with $+\boldsymbol{k}_{A}$ direction and non-absorbed throughput light from the beam generating pulse $A$ results in undesirable signal-to-noise ratios.

Currently, the three surviving different phase matching conditions I, II, and III point in spatially different directions, while maintaining the same time ordering. It is experimentally 
difficult and expensive to acquire data in all of these directions (simultaneously or individually) nor is it necessary. With the right geometry, presently a box, and changing the time ordering, it is possible to have each of these phase matching conditions point in the same spatial direction while still being able to maintain the critical functionality of independently controlling characteristics of each of the three beams (timing, polarization...). This is not possible in twobeam FWM experiments, which have phase matchings such as $2 k_{1}-k_{2}$ where two interactions must occur from a single pulse. Here, there is only one possible time delay to vary and the two contributions from $k_{1}$ will always have the same polarization, for example. Therefore, in the present work the phase matching conditions are $\boldsymbol{k}_{s i g, I}=-\boldsymbol{k}_{A}+\boldsymbol{k}_{B}+\boldsymbol{k}_{C}, \boldsymbol{k}_{s i g, I I}=+\boldsymbol{k}_{B}-\boldsymbol{k}_{A}+$ $\boldsymbol{k}_{C}$, and $\boldsymbol{k}_{\text {sig,III }}=+\boldsymbol{k}_{B}+\boldsymbol{k}_{C}-\boldsymbol{k}_{A}$, which all point in the $-\boldsymbol{k}_{A}$ direction. The experimental realization is detailed in section 3.2 MDCS Setup.

Define the time period between the first and second pulses as $\tau$, the time between the second and third pulse as $T$, and from the third pulse onward as t. Heterodyne measurement (the signal is interfered with a reference- also in Chapter 3 Experimental and Technical Background Information) with phase control allows for measurement of both amplitude and phase information. Thus the complex spectra, detected along their respective phase matching directions, are $[49,113,116]$ :

$$
\begin{gathered}
S_{I}(t, T, \tau)=i R_{I}^{(3)}(t, T, \tau) \mathcal{E}_{C}^{+} \varepsilon_{B}^{+} \varepsilon_{A}^{-} e^{i\left(\omega_{C}+\omega_{B}-\omega_{A}\right) t} e^{i\left(\omega_{B}-\omega_{A}\right) T} e^{-i \omega_{A} \tau} \\
S_{I I}(t, T, \tau)=i R_{I I}^{(3)}(t, T, \tau) \varepsilon_{C}^{+} \varepsilon_{A}^{-} \varepsilon_{B}^{+} e^{i\left(\omega_{C}-\omega_{A}+\omega_{B}\right) t} e^{i\left(-\omega_{A}+\omega_{B}\right) T} e^{+i \omega_{B} \tau} \\
S_{I I I}(t, T, \tau)=i R_{I I I}^{(3)}(t, T, \tau) \varepsilon_{A}^{-} \varepsilon_{C}^{+} \varepsilon_{B}^{+} e^{i\left(-\omega_{A}+\omega_{C}+\omega_{B}\right) t} e^{i\left(+\omega_{C}+\omega_{B}\right) T} e^{+i \omega_{B} \tau}
\end{gathered}
$$

Dephasing can be phenomenologically to the terms constituting each signal. The terms within the response function will determine what rates and time periods must be applied and are particular to each sample, though many similarities do exist across samples.

\subsection{Double-sided Feynman Diagrams}

Double-sided Feynman diagrams are useful graphical diagrams meant to ease calculation of the commutators in the response function, $R^{(3)}(t, T, \tau)$, which generally has many terms and enable intuitive interpretation of 2D spectra without actually explicitly writing the response 
function. Each Feynman diagram represents a term in the response function and necessary assumptions must be made to explicitly write the response function when phenomenologically adding effects to each term. Therefore, while the explicit response function may not accurately reflect the real system's behavior, the Feynman diagram is more "correct" because it is drawn from the general form of the response function with fewer assumptions about the dephasing mechanisms. I will present the rules for the diagrams, and then proceed with a simple example before returning to the FWM signals. In short, the diagrams track the "quantum pathways" through the density matrix as the system is transformed sequentially by each light-matter interaction. Consider a two state system with a ground and excited state, $|0\rangle$ and $|1\rangle$.

1. Time flows from the bottom upwards and two vertical lines represent the ket and bra of the density matrix. A valid quantum pathway must begin and end with a population, i.e. in a diagonal entry of the density matrix, as coherences are not steady states. The system typically starts in the ground state, but may end in a different state. Sometimes the initial and final states are supressed for brevety.

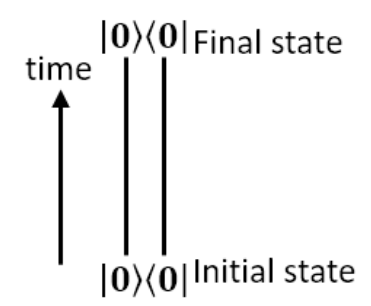

Figure 1 Double-sided Feynman diagram for step 1

2. Any light-matter interaction is represented by an arrow. An arrow pointing inwards will increment the ket or bra and one pointing outwards will decrement it. Arrows pointing in different directions impart different momentum and phase. An arrow to the right represents $e^{i(\boldsymbol{k} \cdot \boldsymbol{r}-\omega t)}$ and to the left $e^{-i(\boldsymbol{k} \cdot \boldsymbol{r}-\omega t)}$. 


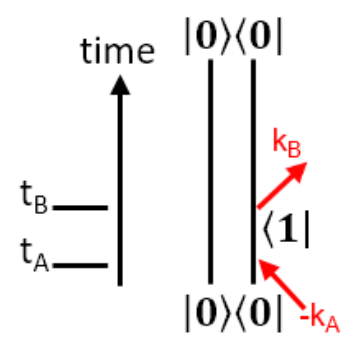

Figure 2 Double-sided Feynman diagram for step 2 showing left and right pointing arrows

3. The light-matter interaction responsible for emission of the signal is different in nature from the driving fields, as it results from the expectation value of the dipole moment operator, $\operatorname{Tr}(\mu \rho)$, and is usually drawn with a different style arrow. A dotted one will be used in this work.

4. The emitted signal will have a frequency and wavevector that is the sum of the input fields' wavevectors and frequencies.

5. Each diagram has a sign associated with it, $(-1)^{m}$ where $m$ is the number of operations from the right. This is because interactions from the right originate from the negative term in the commutator. Since the signal interaction is not part of a commutator, it is not accounted in $m$. Though in this work, the signal will always emerge from the left side.

\section{Example: The linear (coherent) response}

To demonstrate this formalism's utility, consider the linear response of the above mentioned two state system. The response function $\left\langle\mu\left(t_{A}\right) \mu_{A}(0) \rho(-\infty)\right\rangle$ can be constructed as:

1. Nothing happens until $t=0$ when the fist exciting photon arrives and generates a $\rho_{10}$ coherence in the system, with probability proportional to the dipole moment between the two states $\rho_{10}(0) \propto \mu_{10}$ (but squared, of course).

2. This off-diagonal density matrix element will evolve in time as $\rho_{10}\left(t_{A}\right)=$ $\frac{i}{\hbar} \mu_{10} e^{-i \frac{\left(E_{1}-E_{0}\right)}{\hbar} t_{A}} e^{-\gamma t_{A}}$, where the homogeneous dephasing rate $\Gamma$ has been phenomenology added.

3. At time $t_{A}$, the coherence emits a light field, with probability again proportional do the transition matrix element between $|0\rangle$ and $|1\rangle$. The response function is $R^{(1)}(t) \propto$ $\mu_{10}^{2} e^{-i \frac{\left(E_{1}-E_{0}\right)}{\hbar} t} t_{A} e^{-\Gamma t_{A}}$. The Feynman diagram for this is: 


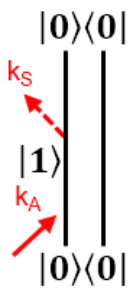

Figure 3 Double-sided Feynman diagram for step 3 showing absorption followed by emission The complex conjugate of this diagram (i.e. the other half of the commutator) is an equally valid pathway, but contains redundant information and is usually not drawn, by convention.

\subsection{Two-Dimensional Correlation Spectroscopy}

Now that we have developed a general form of the detected FWM, we will proceed with developing the formalism further specifically in the context of two-dimensional correlation spectroscopy (2DCS), a subset of MDCS. A series of examples with simulated spectra of model systems exhibiting hallmark properties will demonstrate the power of the technique. A general treatment is outside the scope of this work. See, among others, references $[31,113,117]$ for thorough treatments.

Different pulse time orderings and different scanned time delays result in a rich variation of information that can be extracted from a system. The notation $S_{I}\left(\omega_{\tau}, T, \omega_{t}\right)$ (for example) describes the type of scan/2D spectrum, resulting from phasing matching $k_{I}$, and to communicate which time delays were varied. A frequency as $\omega_{x}$ indicates delay $x(x=t, T$, or $\tau)$ was scanned and Fourier-transformed to the frequency domain whereas a remaining time period indicates it was held constant. 2DCS scans two of the delay times. Though, all delay times available can be scanned- only (very tractable) technical details may need to be overcome. The time delay which is not scanned is normally set to a nonzero constant value, usually $100 \mathrm{fs}$ (one pulse width), to ensure strict time ordering because of possible contributions from other scan types but short enough to minimize contributions from incoherent energy relaxation, which can occur at large values of $T$ [40]. In most spectra, a diagonal plots the equal-absorption-andemission line to illustrate where intra-action features would lie. 


\subsubsection{One-Quantum Rephasing Spectra, $S_{I}\left(\omega_{\tau}, T, \omega_{t}\right)$}

By far the most common type of scan is an $S_{I}\left(\omega_{\tau}, T, \omega_{t}\right)$. It has the phase-matching condition $\boldsymbol{k}_{s}=-\boldsymbol{k}_{A}+\boldsymbol{k}_{B}+\boldsymbol{k}_{C}$ and will also be the focus of much of the current work. It is also a one-quantum (1Q) scan because the all of the observed transitions have energies of about one quantum of the excitation field.

Consider a rephasing experiment qualitatively visualized three ways: an energetic, temporal, and spatial picture as in Figure 4 for a two state system with a ground $|0\rangle$ and excited state $|1\rangle$.

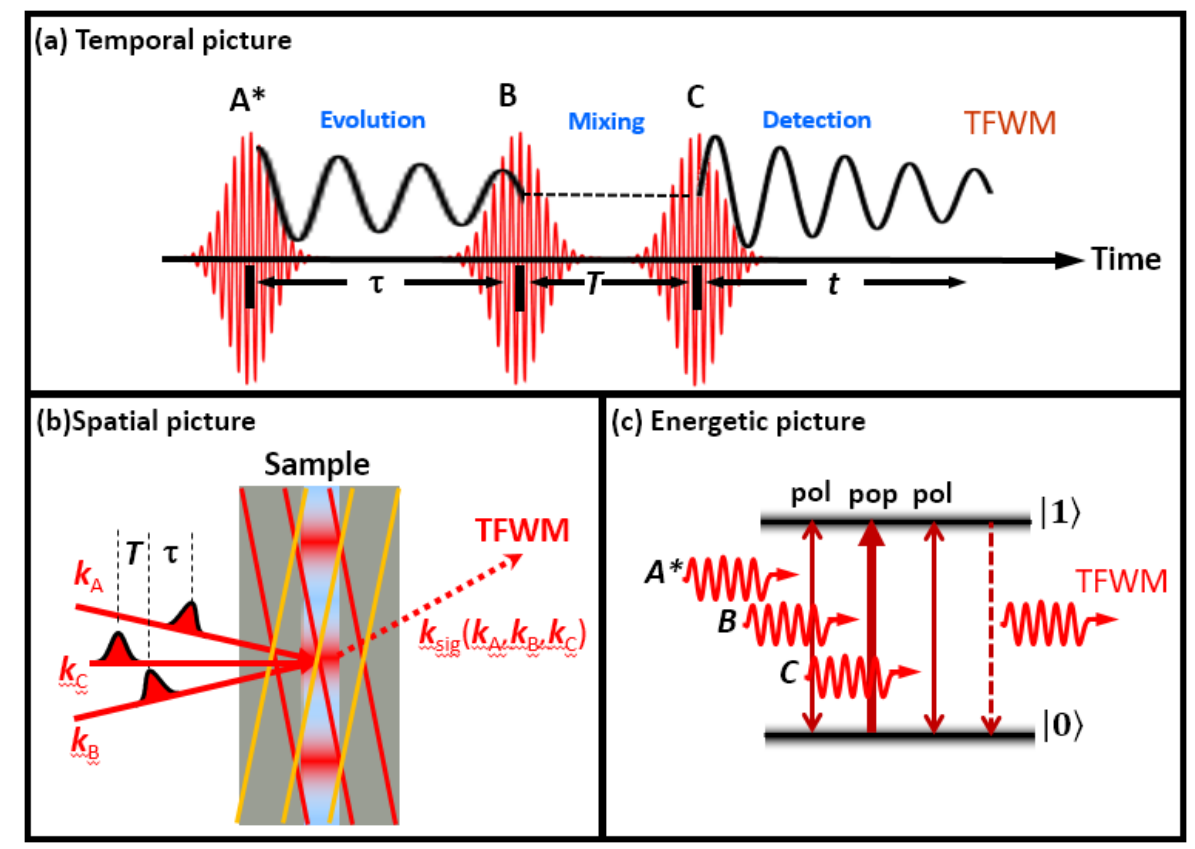

Figure 4 (a) Temporal (b) spatial (b) energetic schematic picture of rephasing experiment As discussed in the previous sections, this signal is assumed to only result from the three pulses (we are only considering the third-order response) and within the rotating-wave and impulse (Dirac pulse envelopes) approximations. We then use the following terminology to discuss the system's evolution. The first pulse, $A^{*}$, arrives and creates a coherent superposition laka polarization aka coherence) between the ground and excited state, as indicated by the oscillating black line in Figure 4 (a) and the first double sided arrow between the two states in Figure 4 (c). In the spatial picture Figure 4 (b) the pulse is a plane wave, whose phase fronts are indicated by 
the solid yellow lines moving across the sample. After time $\tau$, the second pulse, $B$, then creates a population in either the ground or excited state, whose time dependence (relaxation rate) is slow compared to the coherence, as indicated by the flat line in Figure 4 (a). If there were more states energetically nearby small coherences between them could exist and therefore $T$ is sometimes called the population "mixing time." Spatially in Figure 4 (b), this population manifests as an electron, say, population grating generated as a result of pulses $A$ and $B$ interfering at the sample. In the energetic picture Figure 4 (c) the single sided arrow after pulse B represents a population driven to the excited state, though it could equally drive, bleach, the ground-state. After time T the third pulse, $C$, converts the population into a radiating polarization, the transient four-wave mixing (TFWM) signal during period t. Spatially visualized as the signal self-diffracting off of the before mentioned population grating in the phase matched direction. Energetically, after emission the system is returned to the ground state and the sequence is over. This sequence would represent one data point (not a complete 2D spectrum), and it would be repeated each time a time delay is incremented ( $10^{3}$ data points) until the respective time delay is so long that the system fully decoheres before the arrival of the next pulse, resulting in no TFWM signal. Afterwhich a Fourier transform transforms the 1D spectrally resolved-FWM (SR-FWM) spectra as a function of time delay into a 2DCS spectrum.

The transient FWM signal exhibits a photon echo for inhomogeneously broadened systems. This terminology is lifted from and the physics analogous to the spin echo measurements in NMR. Because the phase accrued as a result of the evolution after the first (and conjugated) pulse as a result of inhomogeneity during the first period $\tau$ is halted and undone or "rephased" from the combined effect of the second two unconjugated pulses. Consequently, there is a delay after the final pulse before the signal, the "echo", is emitted. For this reason, it is frequently referred to as a photon-echo or rephasing scan. While the inhomogeneous dephasing can be rephased, homogenous dephasing cannot. In the time domain, the echo will be weaker with increasingly longer $\tau$ time delays. As a result, rephasing scans allow for clear unambiguous separation of homogeneous and inhomogeneous linewidths. This reason alone has made it a common and useful tool.

\section{Example: Three-state, uncoupled system}


Consider an $S_{I}\left(\omega_{\tau}, T, \omega_{t}\right)$ scan of a three-state system with two excited states that share a ground state, however do not couple through the ground state:
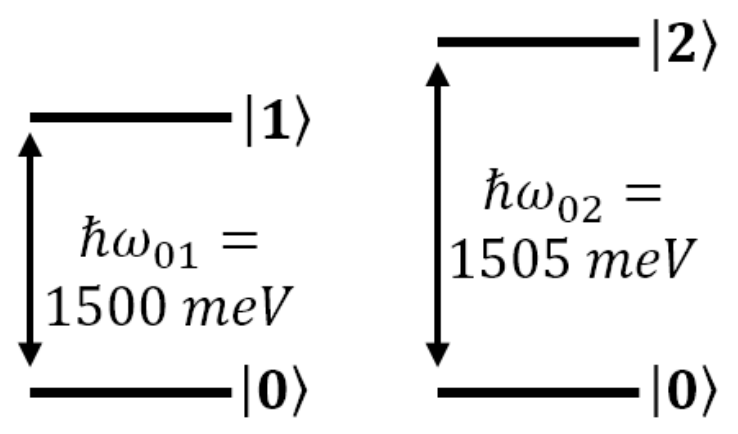

Figure 5 Three-state, uncoupled system with transition energies $\hbar \omega_{01}=1500 \mathrm{meV}$ and $\hbar \omega_{02}=$ $1505 \mathrm{meV}$ between the ground (zeroth) and first and second excited states, respectively.

The transition energies for the first and second exited states are $\hbar \omega_{01}=1500 \mathrm{meV}$ and $\hbar \omega_{02}=$ $1505 \mathrm{meV}$, respectively, where the subscript indicates between which two states the transition occurs. Also let the dipole moments be equal, $\mu_{01}=\mu_{02}$. For a rephasing, FWM experiment, the Feynman diagrams for this system are shown in Figure 6. Note that as there is no coupling between the two excited states, the diagrams for the can be grouped into two independent sets; those that only involve transitions with the $1^{\text {st }}$ excited state and those that only involve transitions with the $2^{\text {nd }}$ excited state. Thus the resulting peaks result from intraction, involving only a single transition.
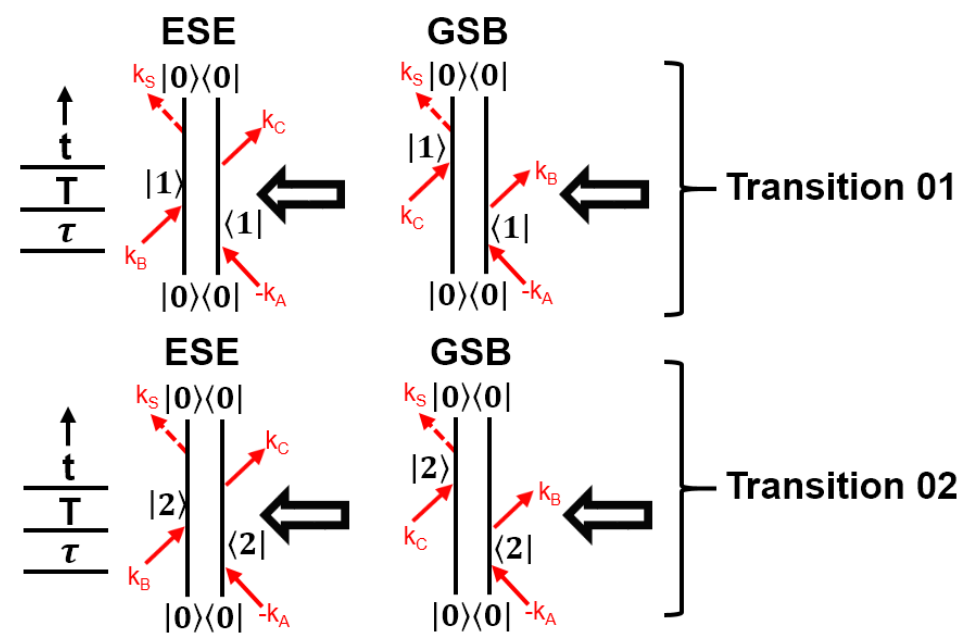

Figure 6 Rephasing Double-sided Feynman diagrams for a two three-state, uncoupled system. Arrows indicate where populations are driven in the excited and ground state for excited state 
emission (ESE) and ground-state bleaching (GSB), respectively. The relevant time periods are illustrated on the left.

The time ordering and phase conjugation of the pulses is the same in all diagrams (pulse $A$, pulse $B$, and lastly pulse $C$ point in their same respective directions) as well as pulse $A$ always acts on the bra and the signal always emits from a ket. All of which is a consequence of the phase matching condition $\left(\boldsymbol{k}_{S}=-\boldsymbol{k}_{\boldsymbol{A}}+\boldsymbol{k}_{\boldsymbol{B}}+\boldsymbol{k}_{\boldsymbol{C}}\right)$ and is thus true for all rephasing diagrams. Furthermore, two types of pathways exist: excited-state emission (ESE) and ground-state bleaching (GSB). ESE pathways generate a population in one of the excited states ( $\rho_{11}$ or $\left.\rho_{22}\right)$ after the second pulse (B), followed by the eventual signal emission from a coherence with that state $\left(\rho_{10}\right.$ or $\left.\rho_{20}\right)$. GSB is similar, however the population is created the ground state $\left(\rho_{00}\right)$. Note that in all of the diagrams, the first pulse generates a coherence with the same state from which it will emit, therefore the energies of these processes are the same which has, among other things, important consequences in the 2DCS spectra. Before displaying the model 2D spectrum, let us write down the response function for the system, constructed with aide of the doublesided Feynman diagrams. Generally (and phenomenologically) in the technique, relaxation mechanisms are added to the systems response during the specific time periods they influence its evolution. This is one of the great advantages of MDCS as it can begin to unravel dynamics which would be otherwise convolved on a 1D spectrum.

Inhomogeneous dephasing is added to the systems response as $e^{-\frac{\sigma_{n m^{2}}^{2}}{2} t_{i}}$ and homogeneous dephasing as $e^{-\gamma_{n m} t_{i}}$, where $t_{i}=\tau, T, t$ and $\sigma_{n m}$ and $\gamma_{n m}$ are the inhomogeneous and homogeneous dephasing rates associated with the $\rho_{n m}$ coherence [62]. Note that inhomogeneous mechanisms lead to Gaussian and homogeneous mechanisms lead to Lorentzian distributions, after Fourier transformation from the time to the frequency domain. Similarly, population relaxation can be included as $e^{-\Gamma_{j} T}$, where $\Gamma_{j}$ is the relaxation rate of the population $\rho_{j j}$. As $\sigma, \gamma \gg \Gamma$, population relaxation is not the dominate mechanism for the "fast" decoherence and strictly speaking only affects populations. Also in a typical rephasing scan, the population mixing time $\mathrm{T}$ is not scanned and set to some finite value to enforce strict time ordering. In section XI will discuss the $S_{I}\left(\tau, \omega_{T}, \omega_{t}\right)$, "capital T" scan. 
For the $\hbar \omega_{01}$ transition, the response function from the ESE and GSB Feynman diagrams is:

$$
2\left(\frac{i}{\hbar}\right)^{3} \mu_{01}^{4} e^{-\frac{1}{2} \sigma_{01}^{2}(\tau-t)^{2}} e^{-\gamma_{01}(\tau+t)} e^{-\Gamma_{1} T}
$$

And similarly for the $\hbar \omega_{02}$ transition. The factor of two accounts for the fact that the ESE and GSB pathways lead to identical response functions, in this case. The signal (recorded in the phase matched direction) is then:

$$
\begin{aligned}
S_{I}(\tau, T, t)=\frac{2}{\hbar^{3}} & |E|^{3} \\
& \times\left[e^{-i \omega_{01}(\tau-t)} \mu_{01}^{4} e^{-\frac{1}{2} \sigma_{01}^{2}(\tau-t)^{2}} e^{-\gamma_{01}(\tau+t)} e^{-\Gamma_{1} T}\right. \\
& \left.+e^{-i \omega_{02}(t-\tau)} \mu_{02}^{4} e^{-\frac{1}{2} \sigma_{02}^{2}(\tau-t)^{2}} e^{-\gamma_{02}(\tau+t)} e^{-\Gamma_{2} T}\right]
\end{aligned}
$$

Furthermore, let $\gamma_{01} \gg \sigma_{01}$ and $\gamma_{02} \ll \sigma_{02}$ such that the two transitions demonstrate two regimes of dephasing, the homogeneous and inhomogeneous limits, respectively. Also let the dipole moments be equal. Then (numerically) Fourier transform to obtain the 2D spectrum:

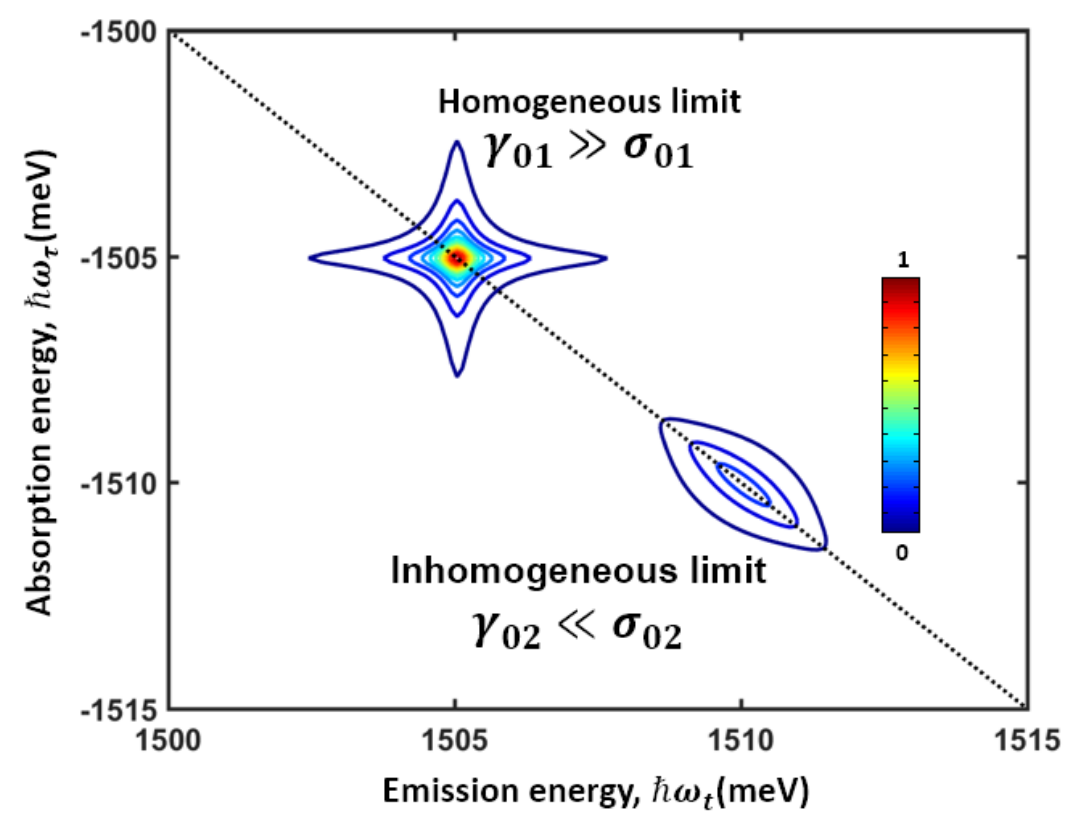

Figure 7 Rephasing amplitude spectrum for a three-state uncoupled system with both homogeneously and inhomogeneously-dominated line shapes. 
The vertical axis corresponds to the absorption photon energy $\hbar \omega_{\tau}$ calculated from the Fourier transform over period $\tau$ and increases downward. It is negative because of the sign convention chosen for the conjugated pulse $\left(-k_{A}\right)$. The horizontal axis is the emission photon energy $\hbar \omega_{t}$ during period $t$ and increases to the right. The diagonal, $\left|-\hbar \omega_{\tau}\right|=\hbar \omega_{t}$, marks where these two energies are equal. Pathways generating populations, diagonal entries in the density matrix, conveniently appear along the drawn diagonal. Similarly, we'll see that pathways involving coherences between transitions, off diagonal elements in the density matrix (besides those with the ground), will appear as off diagonal peaks in the spectrum. The out of plane axis is the intensity and normalized from 0 to 1 , the peak intensity.

For the three-state, uncoupled system there are only peaks along the diagonal, one for each transition. Absorption and emission happen at the same energy. This can be inferred from the Feynman diagrams as the first and last operations on the density matrix involve the same respective transition. The first pulse excites a $\rho_{01}$ coherence which requires an energy of $\hbar \omega_{01}$ and the last interaction, the radiating coherence from $\rho_{10}$ which comprises the signal, must also have an energy of $\hbar \omega_{01}$ (This energy could be written as $\hbar \omega_{10}$, however that would be redundant and not contain any new information). The next clear signature is that of the effect of the different broadening mechanisms on each state. The homogeneously dominated $\hbar \omega_{01}$ transition is star shaped whereas the inhomogeneously dominated $\hbar \omega_{02}$ is elongated along the diagonal in an oval shape. Comparable amounts of homogeneous and inhomogeneous broadening result in intermediate shapes $[29,62]$. This demonstrates a very useful property of 2DCS. Without further quantitative analysis of the spectra, one can determine the transition energies as well as the relative amount of which type of broadening mechanisms are influencing the system. Homogeneous broadening widens the peak in the cross diagonal direction and inhomogeneous broadening tends to widen in the diagonal direction. Therefore, to quantify the linewidths, diagonal and cross slices are taken through the peak centers and fit. Depending on the regime (homogeneous, inhomogeneous, or intermediate), different functions are used. They are summarized in Table 1 and are applied to all types of 2D scans, not just $S_{I}$.

Table 1 2D Amplitude linewidths in varying regimes of broadening $[29,62]$. 


\begin{tabular}{c|c|c} 
& Slice & \multicolumn{1}{c}{$\begin{array}{c}\text { Amplitude Spectrum } \\
\text { Function, FWHM Width }\end{array}$} \\
\hline \multirow{2}{*}{ Inhomogeneous limit } & Cross Diagonal & Lorentzian, $2 \gamma$ \\
\cline { 2 - 3 } & Diagonal & Gaussian, $2 \sqrt{2 \ln 2 \sigma}$ \\
\hline \multirow{2}{*}{ Homogeneous limit } & Cross Diagonal & Lorentzian, $2 \gamma$ \\
\cline { 2 - 3 } & Diagonal & Lorentzian, $2 \gamma$ \\
\hline \multirow{2}{*}{ Intermediate limit } & Cross diagonal & Voigt, $2 \gamma$ (Lorentzian component) \\
\cline { 2 - 3 } & Diagonal & Voigt, $2 \sqrt{2 \ln 2 \sigma}$ (Gaussian component)
\end{tabular}

\section{Example: Four-state, coupled "V" system}

Increasing the complexity, consider now the three-state system from the previous example but with interstate coupling allowed. States $|1\rangle$ and $|2\rangle$ will couple through the ground state $|0\rangle$, as indicated in the energy diagram in Figure 8 by the double-sided arrows for the two transitions making a "V" shape. Also allow for an additional state, $\left|1_{B}\right\rangle$, that is a doubly excited state of $|1\rangle$ only. The nature of $\left|1_{B}\right\rangle$ is that it is a bound state of two particles excited to the $|1\rangle$ state. Selection rules apply and for simplicity we will assume we are injecting the correct momentum for binding to occur. The total energy of $\left|1_{B}\right\rangle$ is $2 \hbar \omega_{01}-\Delta$, where $\Delta$, is the binding energy of the $|1\rangle$ pair. Let $\Delta=2 \mathrm{meV}$.

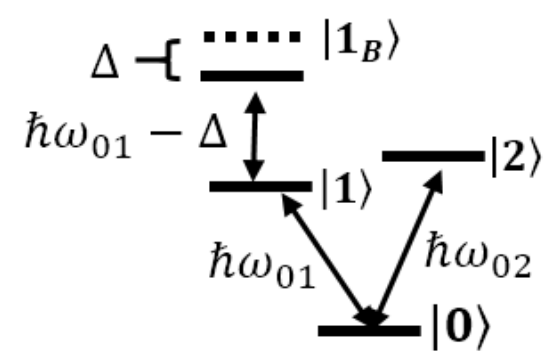

Figure 8 Energy diagram for a four-state system having two coupled singly excited states, and one doubly excited state of $|1\rangle$.

There are nine quantum pathways which can contribute to the rephasing spectrum, as shown in Figure 9. The diagrams in (a), (b), (e), and (f) are the same as those from the previous example. There are two new GSB and two new ESE diagrams as well as an excited state 
absorption (ESA) diagram now. The four new GSB and ESE terms, (c)-(d) and (e)-(h), contain interstate coupling now which will result in off diagonal peaks. For example, in the GSB diagram (c), first a $\rho_{01}$ coherence is generated and lastly the signal emits from a $\rho_{20}$ coherence. Looking at a new ESE diagram, (g), the same sort situation occurs with an initial $\rho_{01}$ and final $\rho_{20}$. However during period T a $\rho_{21}$ coherence is generated. This type of coherence is sometimes called a Raman coherence because it is too low of an energy to be radiative. These sorts of interstate quantum pathways are only possible when coupling occurs between states.

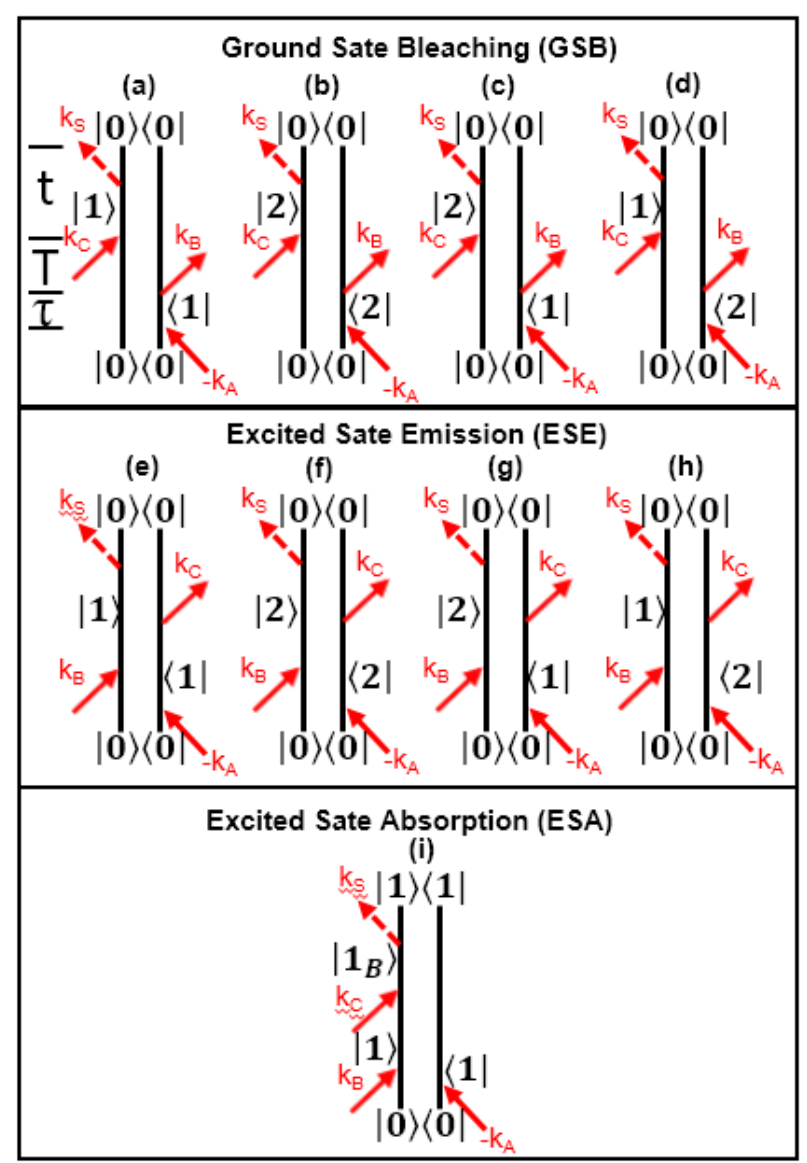

Figure 9 Quantum pathways for the system in Figure 8, (a)-(d) ground state bleaching, (e)-(h) excited state emission, and (i) excited state absorption

The ESA pathway is shown in Figure 9 (i). It accounts for accessing the doubly excited state $\left|1_{B}\right\rangle$, which is done by creating a $\rho_{1_{B} 1}$ coherence after pulse $C$ which radiates leaving the system in a populated state $\rho_{11}$, rather than $\rho_{00}$ as with the GSB and ESE pathways. ESA is therefore a two-quantum contribution to an otherwise one-quantum scan. 
Explicitly writing the response function:

$$
\begin{aligned}
& S_{I}(\tau, T, t) \\
& =\frac{1}{\hbar^{3}}|E|^{3} \times \\
& \times\left[\begin{array}{c}
2 \mu_{01}{ }^{4} e^{-i \omega_{01}(\tau-t)} e^{-\frac{1}{2} \sigma_{01}^{2}(\tau-t)^{2}} e^{-\gamma_{01}(\tau+t)} e^{-\Gamma_{1} T}+ \\
2 \mu_{02}{ }^{4} e^{-i \omega_{02}(\tau-t)} e^{-\frac{1}{2} \sigma_{02}^{2}(\tau-t)^{2}} e^{-\gamma_{02}(\tau+t)} e^{-\Gamma_{2} T}+ \\
\mu_{01}{ }^{2} \mu_{02}{ }^{2} e^{-i\left(\omega_{02} \tau-\omega_{01} t\right)} e^{-\frac{1}{2}\left(\sigma_{02} \tau-\sigma_{01} t\right)^{2}} e^{-\left(\gamma_{02} \tau+\gamma_{01} t\right)} e^{-\Gamma_{2} T}+ \\
\mu_{01}{ }^{2} \mu_{02}{ }^{2} e^{-i\left(\omega_{01} \tau-\omega_{02} t\right)} e^{-\frac{1}{2}\left(\sigma_{01} \tau-\sigma_{02} t\right)^{2}} e^{-\left(\gamma_{01} \tau+\gamma_{02} t\right)} e^{-\Gamma_{1} T}+ \\
\mu_{01}{ }^{2} \mu_{02}{ }^{2} e^{-i\left(\omega_{02} \tau-\omega_{01} t\right)} e^{-\left(\gamma_{02} \tau+\gamma_{01} t\right)} e^{-\frac{1}{2}\left(\sigma_{02} \tau-\sigma_{01} t\right)^{2}} e^{i \omega_{12} T-\frac{1}{2} \sigma_{12}^{2} T^{2}-\gamma_{12} T}+ \\
\mu_{01}{ }^{2} \mu_{02}{ }^{2} e^{-i\left(\omega_{01} \tau-\omega_{02} t\right)} e^{-\left(\gamma_{01} \tau+\gamma_{02} t\right)} e^{-\frac{1}{2}\left(\sigma_{01} \tau-\sigma_{02} t\right)^{2}} e^{i \omega_{12} T-\frac{1}{2} \sigma_{12}^{2} T^{2}-\gamma_{12} T}+ \\
\mu_{01}{ }^{2} \mu_{1_{B} 1}{ }^{2} e^{-i \omega_{01} \tau} e^{i\left(\omega_{01}-\frac{\Delta}{\hbar}\right) t} e^{-\frac{1}{2}\left(\sigma_{01} \tau-\sigma_{1}{ }^{1} t\right)^{2}} e^{-\left(\gamma_{01} \tau+\gamma_{1 B^{1}} t\right)} e^{-\Gamma_{1} T}
\end{array}\right]
\end{aligned}
$$

The first two terms result from Feynman diagrams (a), (b), (e), and (f) as in the previous example. The third and fourth terms result from the GSB (c) and (d) diagrams and the fifth and sixth terms result from the ESE diagrams (g) and (h). Notice that for these two ESE terms the equation tracks the system's more complicated response during period $\mathrm{T}$, where a coherence is driven rather than a population. This coherence evolves in time at rate of $\omega_{12} \ll \omega_{01}, \omega_{02} . I$ assume there is some (albeit slow) homogeneous and inhomogeneous dephasing occurring, characterized by $\gamma_{12}$ and $\sigma_{12}$, respectively. Though realistically, $\mathrm{T}$ is too short in a rephasing scan $\left(\ll \frac{1}{\omega_{12}}, \frac{1}{\gamma_{12}}, \frac{1}{\sigma_{12}}\right)$ for these to have an effect on most systems $[118,119]$. The seventh term results from the ESA diagram (i). For purposes of plotting the model spectrum I will assume that the $\rho_{1_{B} 1}$ coherence experiences the same amounts of homogeneous and inhomogeneous dephasing as the $\rho_{01}$ coherence and that the dipole moment $\mu_{1_{B} 1}$ is $25 \%$ less than $\mu_{01}$ to reflect the realistic situation that this peak is weaker than the other diagonal peaks in many measurements. The resulting 2D spectrum is shown in Figure 10. 


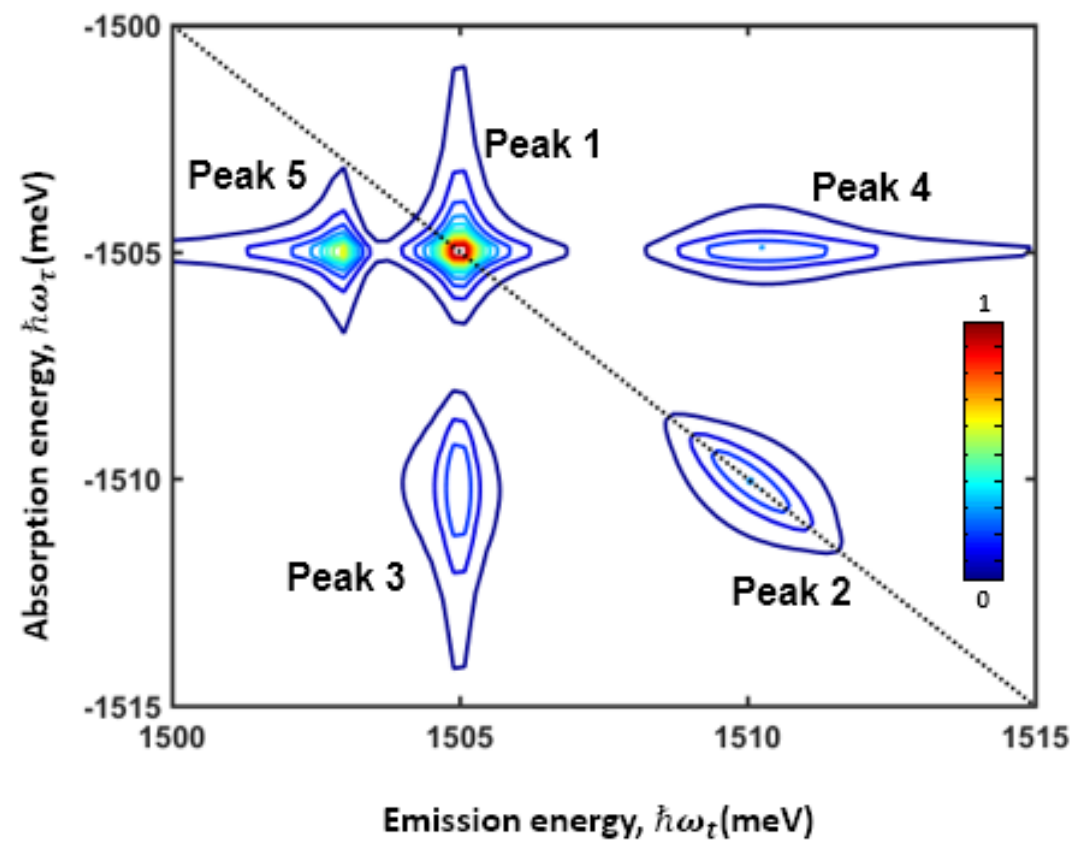

Figure 10 Rephasing amplitude spectrum for a four-state system with coupling.

Five peaks are now present. The peaks on the diagonal, peaks 1 and 2, are the same as in the previous example, resulting from interactions involving only one transition. The off diagonal peaks, 3 and 4, result from intraction amongst the transitions and are clear, unambiguous evidence of coherent coupling. Peak 3 is described by Feynman diagrams Figure 9 (d) and (h) because it lies at the absorption energy of $|2\rangle$ and emission energy of $|1\rangle$, which corresponds to the first interaction (pulse A) generating a $\rho_{02}$ coherence and the last interaction emitting from a $\rho_{10}$ coherence in the Feynman diagrams. Similarly, peak 4 is described by Feynman diagrams Figure 9(c) and (g). Also note that the off diagonal terms are symmetric in shape and intensity in this simple model, as the density matrix is Hermitian $\left(\rho_{m n}=\rho_{n m}^{*}\right)$.

Peak 5 results from the ESA Feynman diagram Figure 9 (i). Bound states have the distinctive property that they are offset from the diagonal along the emission axis by their binding energy. The reason is straight forward: The first and third interactions supply the two quanta necessary to form the bound two-particle state and then the last, radiating interaction decays with an energy of one of its constituent particles, less the binding energy. Since the first and last time periods are Fourier transformed, this manifests as absorption at the single particle energy and an offset in emission energy by the binding energy. This bound state, a two-quantum state, 
does not decay to the ground state because such a transition is forbidden by dipole selection rules. Though physically, dark transitions cannot be ruled out and may occur with a (vanishingly) small probability, of course. As a result of this assumption, all higher quantum states are mapped onto one-quantum transitions. Further exploiting selection rules allows one to explore variations in the system's evolution as a result of controlling which transitions occur. For example, a bound two-particle state generally results from the pairing of two particles with opposite spin. By only injecting particles with one spin state, one could "turn off" the two-particle peak or conversely, encourage it by injecting alternate spin states. This is easily accomplished experimentally by varying the exciting beams' polarizations.

\subsubsection{Zero-Quantum Spectra, $S_{I}\left(\tau, \omega_{T}, \omega_{t}\right)$}

To investigate the low-energy coherences which may exist between single-quantum states, for example $\rho_{12}$ from the previous example, $\mathrm{T}$ and $t$ are scanned and Fourier transformed while holding $\tau$ constant, which is rephasing and has the same phase matching condition, $\boldsymbol{k}_{S}=$ $-\boldsymbol{k}_{A}+\boldsymbol{k}_{B}+\boldsymbol{k}_{C}$, and same Feynman diagrams as $S_{I}\left(\omega_{\tau}, T, \omega_{t}\right)$. These are sometimes called zero quantum (0Q) scans because of the small energy coherences investigated or "capital T" scans because $T$ is scanned rather than $\tau$.

\section{Example: Three-state, coupled " $V$ " system, $S_{I}\left(\tau, \omega_{T}, \omega_{t}\right)$}

Figure 11 shows an example of a capital T scan spectrum acquired on a real system very similar to the three state system with coupling from the earlier model [22]. The vertical axis is the energy associated with $\hbar \omega_{T}$ and denoted as the "Raman Coherence Energy" and the horizontal axis is the same emission $\hbar \omega_{t}$ energy as before. 


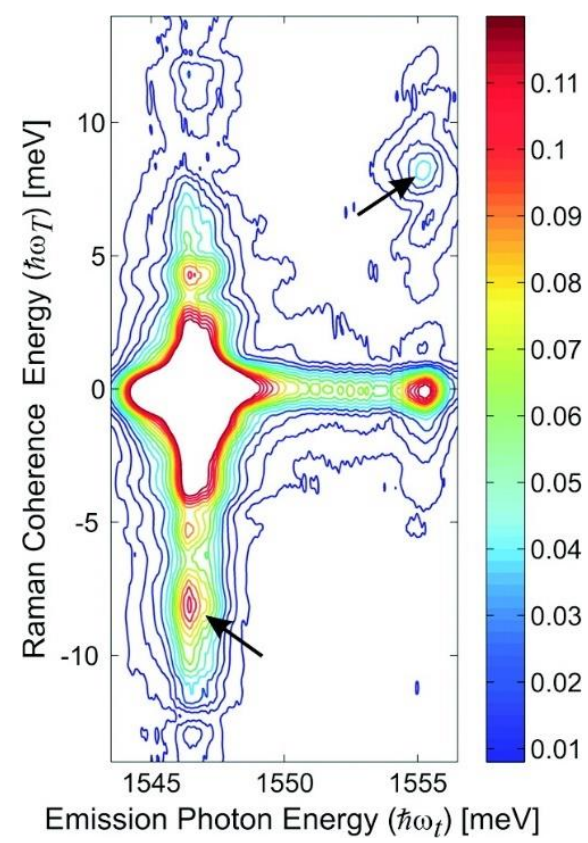

Figure 11 Amplitude $S_{I}\left(\tau, \omega_{T}, \omega_{t}\right)$ spectrum of a three-state system. The color intensity scale is set to saturate for the zero energy Raman peaks in order to emphasize the Raman peaks which, indicated by the arrow. Reproduced from reference [22].

The central peaks at $\hbar \omega_{T}=0$ result from all of the GSB and ESE pathways that do not involve a Raman-like coherence (such as $\rho_{12}$ from the previous example) and would dominate the spectra such that the Raman peaks wouldn't be visible because there are many more diagrams contributing to the central peaks and the evolution during $T$ is generally small (thus weak). Therefore, the spectra are plotted with an intensity color scale that saturates at a fraction of the central peaks' intensities, allowing weaker peaks to be seen. The off-diagonal Raman peaks appear at \pm the difference energy between the states, which are separated by about $8 \mathrm{meV}$. The $+8 \mathrm{meV}$ corresponds to the Feynman diagram Figure 9 (g) and the $-8 \mathrm{meV}$ to diagram (h). Vertical slices provide linewidths to estimate the broadening due to the Raman coherences. Excitation density dependent measurements of the Raman linewidths can determine whether the fluctuations in energy of the two states are correlated or anti-correlated, meaning whether the energies shift in the same or opposite directions in time as a result of coherence $[22,120]$.

\subsubsection{One-Quantum Non-Rephasing Spectra, $S_{I I}\left(\omega_{\tau}, T, \omega_{t}\right)$}

Observing a systems response without rephasing the inhomogeneous dephasing is accomplished with a non-rephasing scan. The time ordering is changed, and the phase matching 
becomes $\boldsymbol{k}_{S}=\boldsymbol{k}_{B}-\boldsymbol{k}_{A}+\boldsymbol{k}_{C}$. The $\tau$ and $t$ delays are scanned and Fourier transformed while holding $\mathrm{T}$ constant. As a result of the phase-conjugated pulse arriving at the sample second, the inhomogeneous phase evolution accrued after the first (and unconjugated) pulse is not unwound by the latter two pulses, merely halted by the second (and conjugated) pulse and set again in motion in the same phase direction by the last (and unconjugated) pulse. As a result, the intensity of the peaks tends to be weaker than that of an $S_{I}$ scan.

Example: Three-state, coupled "V" system, $S_{U}\left(\omega_{\tau}, T, \omega_{+}\right)$

Figure 12 shows an example of an $S_{I I}$ scan and its corresponding $S_{I}$ scan [47]. The peak intensities are comparable because this particular system is an atomic gas with little inhomogeneous broadening. Also note that the vertical axis is positive in the $S_{I I}$ rather than negative as for the $S_{I}$ case and so the diagonal is also flipped. 


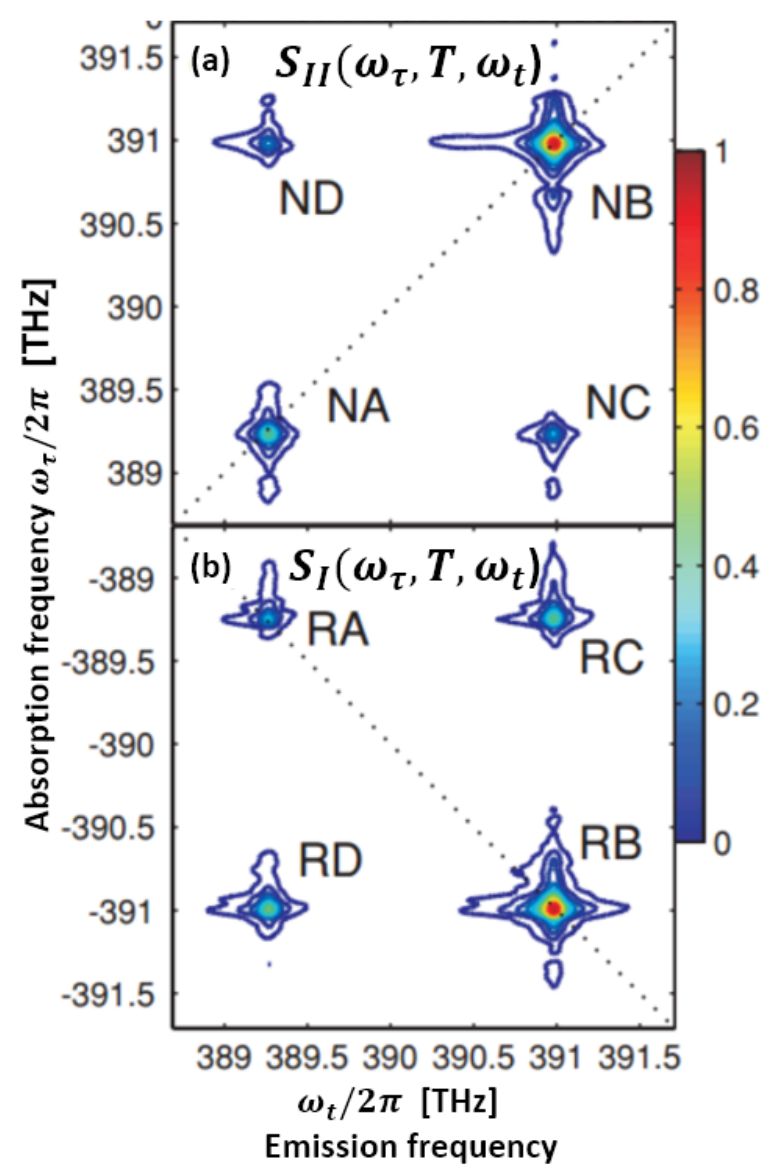

Figure 12 (a) Non-rephasing and (b) rephasing amplitude spectra for a three-state coupled system. The four peaks, $A-D$, are prefaced with an " $N$ " or " $\mathrm{R}$ " to indicate which type of scan generated it. Reproduced from reference [47].

The Feynman diagrams for nonrephasing peaks are shown in Figure 13. Note that the time ordering in them reflects that the phase conjugated pulse is second. Also there are three diagrams contributing to the diagonal peaks and one to the off diagonal peaks, whereas an $S_{I}$ scan has two diagrams for each peak. All things being equal, this would mean the nonrephasing diagonal peaks would be more intense and the off-diagonal peaks less intense than their rephasing counterparts. 

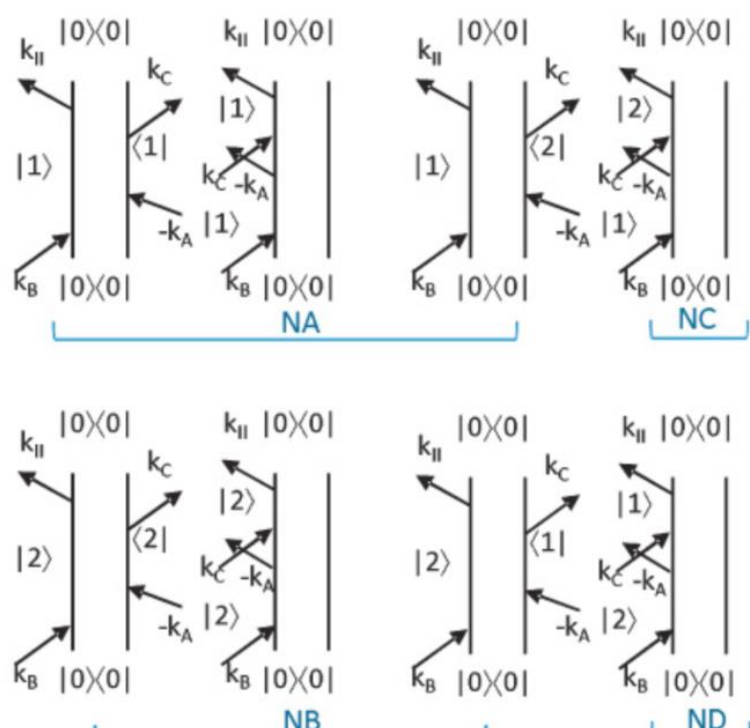

Figure 13 Feynman diagrams for a non-rephasing scan of a three-state coupled "V" system. Reproduced from reference [47].

\subsubsection{Two-Quantum Spectra, $S_{I I I}\left(\tau, 2 \omega_{T}, \omega_{t}\right)$}

With three pulses, it is possible to conduct a non-rephasing, two-quantum (2Q) scan which specifically targets doubly excited states. The phase matching condition is $\boldsymbol{k}_{S}=\boldsymbol{k}_{B}+\boldsymbol{k}_{C}-$ $\boldsymbol{k}_{A}$, where the conjugated pulse is last. Time period $\tau$ is held constant while, $T$ and $t$ are scanned and Fourier transformed. The period $T$ results in a frequency axis of $2 \omega_{T}$ because the first two pulses necessarily drive a two-quantum coherence which evolves during $T$. Two-quantum coherences also dephase more quickly $(2 \omega)$ than single, because they have twice the frequency. This also results in generally weaker spectra than single-quantum scans.

\section{Example: Quantum well, $S_{I U}\left(\tau, 2 \omega_{T}, \omega_{t}\right)$}

Consider as an example a $2 \mathrm{Q}$ amplitude spectrum from a GaAs quantum well sample as in Figure 14 (i) $[67,121]$. The vertical axis is the $2 Q$ frequency resulting from scanning period $T$ and positive. The horizontal axis is the 10 emission frequency, expressed as a detuning from the laser's center frequency $\omega_{0}=368 \mathrm{THz}$. The salient details of the system are that there is a ground state $|g\rangle$ shared by two $1 Q$ states labeled $|H\rangle$ and $|L\rangle$ (they represent two flavors of excitons which exist in GaAs well-a heavy- and light- hole exciton). The frequencies of these transitions are $372 \mathrm{THz}$ and $373.85 \mathrm{THz}$, respectively. The $1 \mathrm{Q}$ states are free to interact as they 
may to form $2 Q$ states. This process is encouraged by cross-linearly polarized excitation which generates up and down spin excitons while minimizing excitation induced dephasing (EID).

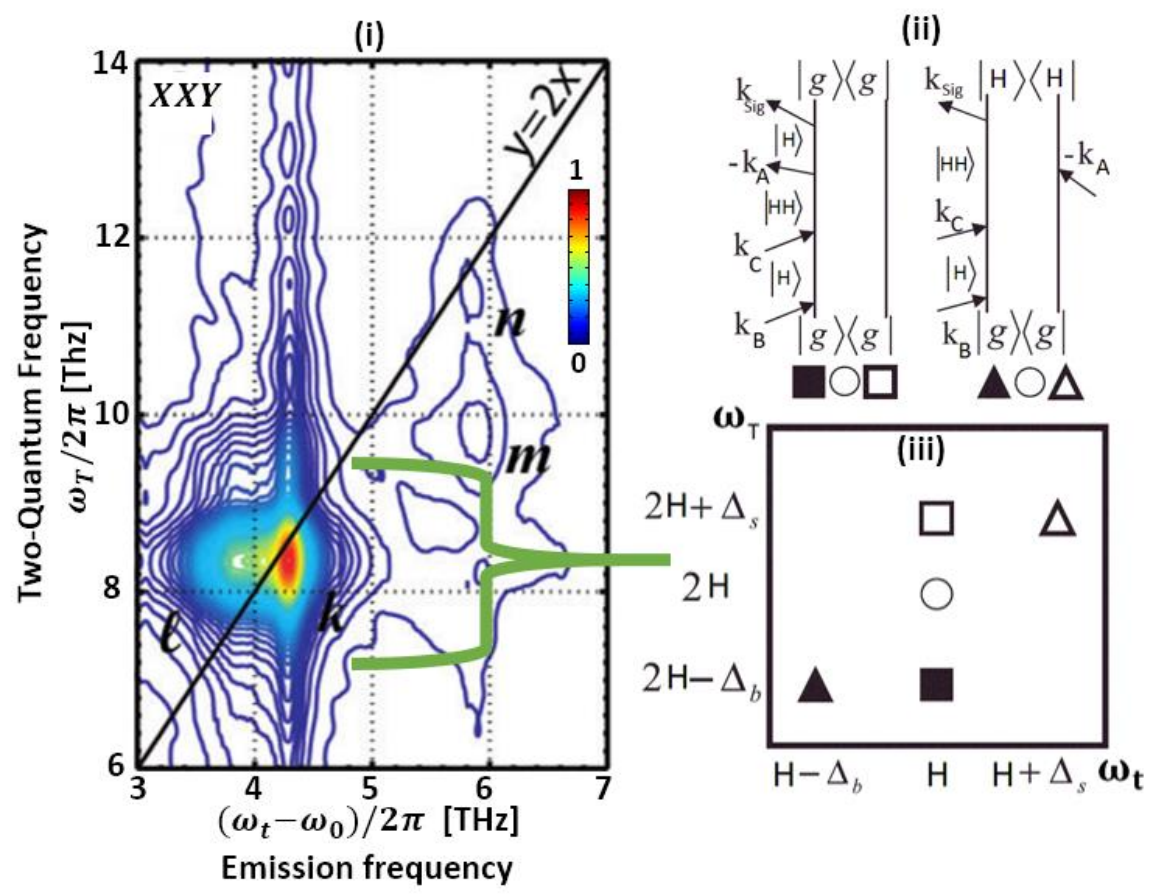

Figure 14 (i) Two-quantum spectrum of GaAs 10 period multi-quantum well sample under crosslinear excitation and generally possible two-quantum (ii) Feynman diagrams applied to a heavy hole states along with (iii) their positions in a $2 Q$ spectrum. Reproduced from references $[67,121]$.

The, in principle, allowed Feynman diagrams as applied to a heavy-hole $2 \mathrm{Q}$ state $|\mathrm{HH}\rangle$ are shown in Figure 14 (ii). The first two interactions, pulses $B$ and C, immediately generate the $2 Q$ coherence. The time delay between them, $\tau$, is held constant at one pulse width to ensure strict time ordering and little evolution. The final pulse, pulse $C$, can convert the groundstate-2Q coherence into a radiating exciton-ground state coherence or excite another excitonic coherence from which the signal radiates from a 2Q-exciton coherence. Depending on the nature of the interaction between the $1 \mathrm{Q}$ states, there are six possible Feynman diagrams possible corresponding to the four peaks in Figure 14 (iii) labeled by squares, circles, and triangles [67]. The axes are the same as the presented spectrum. Solid shapes represent bound states with binding energy $\Delta_{B}$ and hollow ones represent scattering states with scattering energy $\Delta_{S}$. Circles represent correlated but not bound states. 
As the square peaks result from the Feynman diagram with a final radiating excitonground state coherence, which always emit at the same energy because they do not experience the offsets from scattering or binding, these peaks are spread along the vertical, absorption axis but not the horizontal emission axis. Conversely, the triangle peaks are spread along both axes because the final radiating coherence has an energy modified by any binding or scattering. Thus with knowledge of the $1 Q$ energies, one can identify several variations of how two particles may interact as they appear as distinct peaks in 2D spectra.

Completing the example, the peaks can be identified. Peak $(\mathcal{R})$ is a bound heavy hole pair which followed the solid square pathway, peak $(\ell)$ is also a bound heavy hole pair which followed the solid triangle pathway, peak $(m)$ is a bound heavy and light hole pair that also followed the solid square pathway, peak $(\boldsymbol{n})$ is a bound light hole pair also from the solid square pathway. Binding energies can be extracted from the redshifts of the peaks, as well as linewidths from peak slices, and relative oscillator strengths (while also keeping in mind differing absorption cross sections for the states also modify the intensity).

\subsubsection{Real Spectra}

So far we have only discussed the amplitude spectra. However, as the spectra are complex and the experimental implementation preserves phase information, yet more information can be ascertained from the various possible scans by looking at the real or imaginary component. As the real and imaginary components are related by Kramers - Kronig relations, looking at both supplies redundant information and it has become customary to use the real part. Phase sensitive measurements are able to distinguish between microscopic interactions affecting the systems evolution, whereas amplitude only measurements cannot $[21,42,47,58,60,66,122,123]$. In the notation of the coupled three state system example earlier, consider the coherence $\rho_{01}$ with phenomenologically added excitation-induced dephasing $\gamma_{E I D} N \rho_{11}$ and excitation-induced frequency shift (EIS) $\omega_{E I S} N \rho_{11}$, where $N$ is the number density of the oscillators populating the |1) state (generated by optical excitation) [21]:

$$
\frac{d}{d t} \rho_{01}=-\left(\gamma_{01}+\gamma_{E I D} N \rho_{11}\right) \rho_{01}+i\left(\omega_{01}+\omega_{E I S} N \rho_{11}\right) \rho_{01}+\frac{i}{\hbar} \mu_{01} E\left(\rho_{11}-\rho_{00}\right)
$$


Simulated spectra are shown in Figure 15.
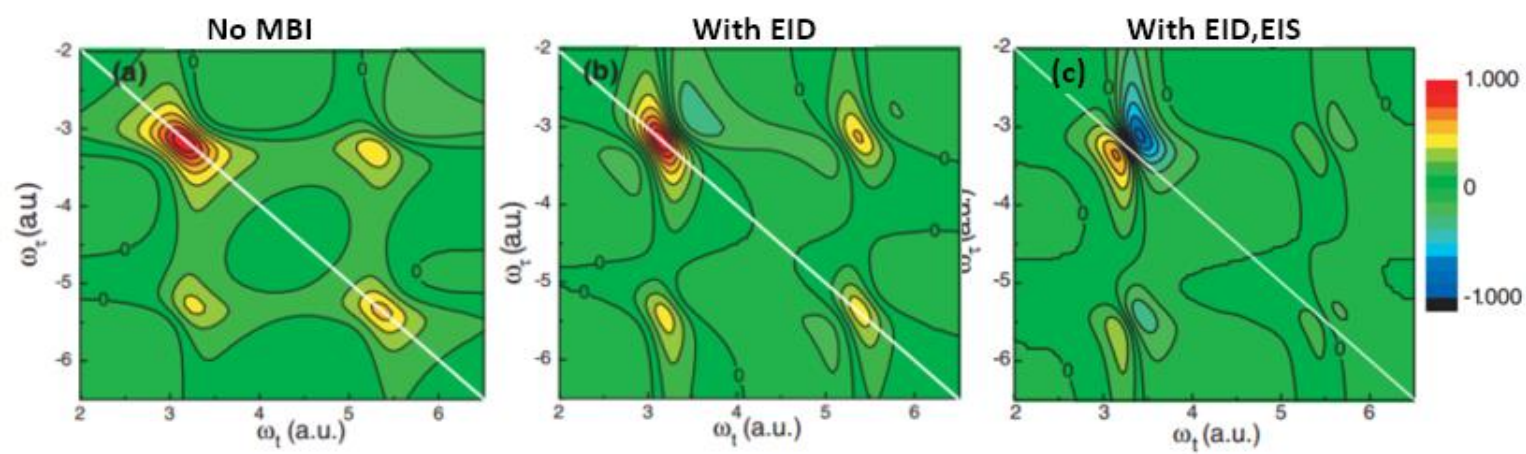

Figure 15 Simulated real rephasing spectra for a coupled "V" system (a) with no many-body interactions, (b) with excitation-induced dephasing, and (c) with excitation induced dephasing and shift. Reproduced from reference [21].

Without many body interactions ( $\mathrm{MBI})$, the model shows purely absorbative type line shapes, Figure 15(a). The introduction of excitation-induced dephasing alters the phase profile, adding negative "twists" at the edges of the peaks, while overall still maintaining absorbative shapes, Figure 15 (b). Adding the excitation-induced shift significantly alters the line shape further from absorbative (peaked at the center of the distribution) to purely dispersive (with negative and positive peaks either side of the center of the distribution), Figure 15 (c). Only with this last addition, does the modeled real spectrum reasonable match that of an actual system, shown in Figure 16 (b) [21]. In additional to different dephasing rates of the two states, different absorbances, as shown in Figure 16(a), under symmetric excitation spectral intensity will also result in asymmetry of the $2 \mathrm{D}$ spectra. Thus capturing the phase of a spectrum enables a sensitive tool to observe and differentiate effects of microscopic interactions which ultimately determine the system's evolution. 


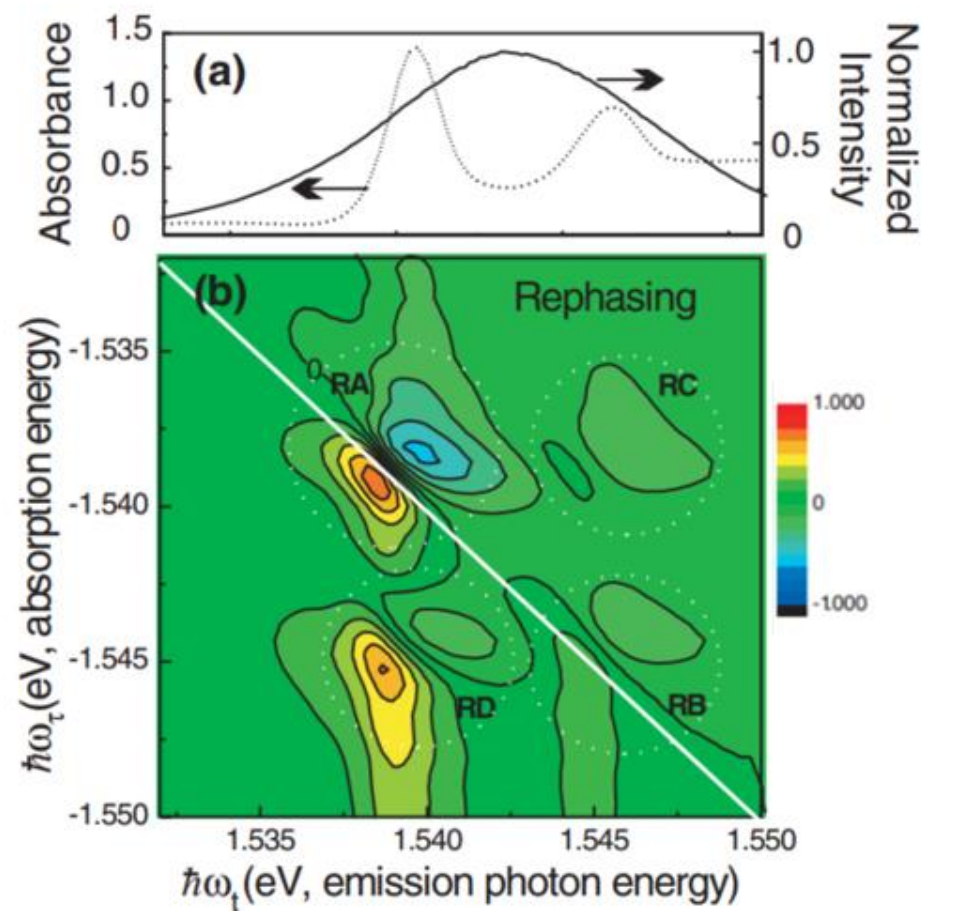

Figure 16 (a) linear absorption and exciting laser pulse spectrum and (b) real rephasing spectrum for an actual coupled "V" system. Reproduced from reference [21].

In summary, 2DCS scans are a powerful tool for determining which transitions are present, coupling, bound, unbound, or scattering as well as their relative amplitudes and deconstructing linewidths into amounts of homogeneous and inhomogeneous broadening. Much of this information can be determined from observing the qualitative features of the spectra-one of the great advantages of the technique 


\section{Chapter 3 Experimental and Technical Background Information}

\subsection{Coherent Light Sources (the lasers)}

The exciting radiation field is supplied by a mode-locked solid-state Ti:sapphire laser that produces $\sim 100-f s$ pulses at a repetition rate of $76 \mathrm{MHz}$, the Coherent MIRA Optima 900-F. It is pumped by a 7 Watt, continuous wave, $532 \mathrm{~nm}$ solid state laser, the Coherent Verde G7. Modelocking is achieved via a perturbation to the cavity which grows, aided by Kerr lensing, to become the soliton pulse. Vibration of a butterfly-shaped glass block perturbs the optical path length inside the laser cavity. This perturbation generates strong power fluctuations. Because the bandwidth is changing as a result of the vibration, momentarily the number of modes in the cavity is fluctuating. When the bandwidth increases, the power spikes, transferring more energy from the gain medium to the electric field modes. Similarly, when the bandwidth drops so too does the energy, and thus the intensity of the beam. A method to discriminate between high and low intensity light and isolate and encourage the high intensity oscillations to achieve a steady state pulsed state is accomplished with Kerr lensing and continuous wave (CW) suppression. Kerr lensing relies on the intensity-dependent index of refraction of the gain medium, sapphire doped with titanium in this case. The Kerr effect is a third-order effect where a materiel's index of refractions depends on intensity of light propagating through it (higher for more intensity light). More formally, the refractive index inside the gain medium can be written as $n=n_{o}+n_{2} I$, where $I=2 n_{o} \epsilon_{o} c|E|^{2}$ is the time average of the light intensity and $n_{2}=\frac{3}{4 n_{o}^{2}} \chi^{(3)}$ the nonlinear index of refraction. Thus this is third order effect and the index actually depends on the square of the applied electric field (i.e. its intensity). [114] This effect is part a wider class of electrooptic class where the material properties (typically the index of refraction) change relatively slowly compared to the high frequency applied fields. The more intense light (which is more pulsed) is focused more than the less intense light (more continuous wave, CW). The CW component can then be physically blocked using an iris or slit placed directly in the beam path. A diagram of this arrangement is shows in Figure 17 where the wider red CW beam is focused less than the higher intensity green pulsed beam and blocked by the hard aperture. [124] 


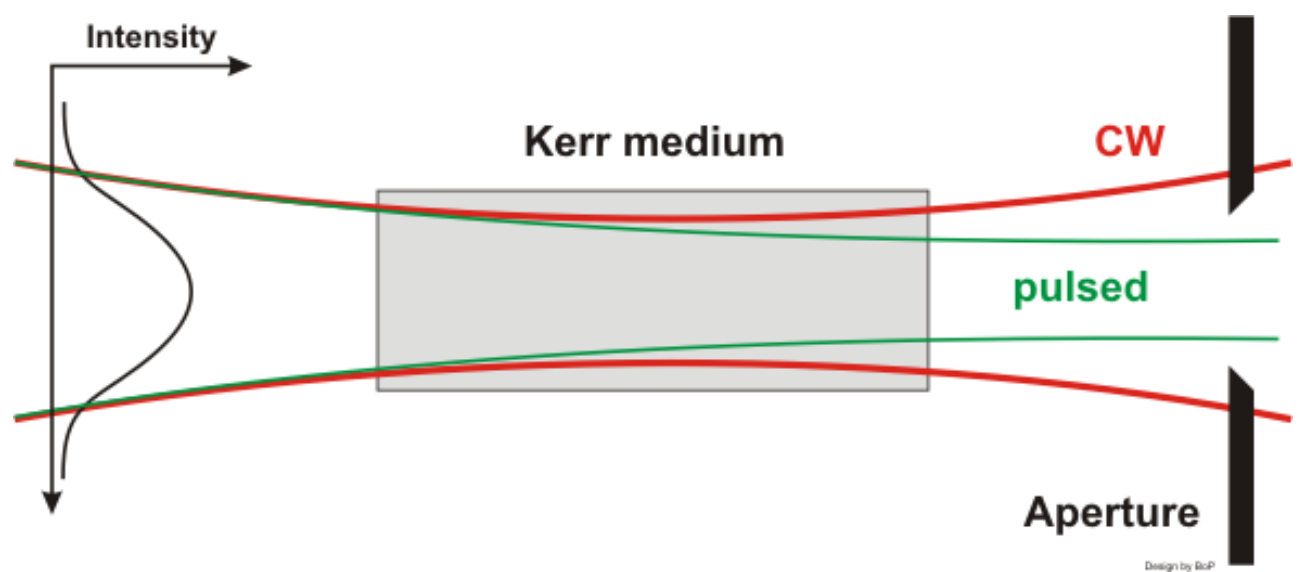

Figure 17 Cartoon diagram of Kerr lens modelocking scheme. Reproduced from reference [124].

The tuning range, the wavelength range over which the laser can operate, is $~ 700-980$ $\mathrm{nm}$, with the current optics and pump laser. Tuning is achieved via birefringent filter (BRF) which acts as a variable wavelength half-wave plate. The principle is that since the cavity is optimized with horizontal polarization owing to the input horizontal polarization from the pump beam, the wavelength which is rotated by the BRF into horizontal polarization is the center wavelength (in the case of ML operation) that survives in the cavity. For a more thorough description of Kerr lensing and electro-optic processes see references $[114,115,125]$ and specifically in the context of lasers see references $[126,127]$.

A CVI Melles Griot 25-LHP-171-249 Helium Neon gas laser is used for metrology. It is pumped electrically and provides $632.8 \mathrm{~nm} \mathrm{CW}$ linearly polarized light at a power of $7 \mathrm{~mW}$. A different wavelength laser is necessary in order to spectrally filter it from pathways which will excite the sample. Other, shorter, wavelengths have been used to increase the phase resolution of the detectors (however it was not demonstrated if this had any scientific benefit) [27].

\subsection{MDCS Setup}

A Multidimensional Optical Nonlinear SpectRometer (MONSTR) is used to create and phase control four identical ultrafast pulses, $A^{*}, B$, and $C$, arranged on the corners of a box, as shown in Figure 18 for a typical rephasing scan. The * indicates phase conjugation with respect to the other pules. The pulses can be independently adjusted to control the delay times $\tau$ and $T$ 
(see inset of Figure 18 for time sequence), as well as the polarization state and power of each beam. The entire setup is depicted in Figure 20 - Figure 28 and can be referred to for the remainder of this section. Figure 24 shows the MONSTR output beams, waveplates, and polarizers to independently control the polarization state of each beam, allowing for exploitation of selection rules.

Excitation irradiances are limited by a variable neutral optical density filter (before they are split into identical pulses by the MONSTR) to ensure the response is in the $\chi^{(3)}$ nonlinear optical regime. Neutral density filters are essential for attenuating pulsed lasers, as non-uniform spectral attenuation would destroy the pulse. The pulses impinge the sample at near-normal incidence ( $\sim 4$ degrees), allowing for spatial selection of the emitted transient TFWM signal emitted in the background-free, phase matched direction. $\boldsymbol{k}_{S}=-\boldsymbol{k}_{\boldsymbol{A}}+\boldsymbol{k}_{\boldsymbol{B}}+\boldsymbol{k}_{\boldsymbol{C}}$, where the ordering of the subscripts indicates the time ordering of the pulses and as stated above, can be varied. The sample resides in an optical cryostation at the focus of a 1:1 telescope and crossing point of the four beams, see Figure 22 and Figure 25. A tracer $(\mathrm{Tr})$ beam pointing in the phase matched direction is used for alignment and blocked for the FWM and 2DCS measurements (such that only three (A, B, C) excite the sample).

As discussed in Chapter 2 Theory of FWM and MDCSin detail, the pulses generate a radiating polarization. This polarization emits a transient FWM that is rotated to vertical polarization (parallel to the spectrometer entrance slit and grating rulings) for maximum detection efficiency in a 1-meter SPEX 1702 spectrometer, see Figure 26. The spectrometer's diffraction grating has 800 plane-ruled grooves $/ \mathrm{mm}$, giving spectral resolution of $.035 \mathrm{meV}$, see Figure 26. The resulting spectrum is recorded by a thermoelectrically cooled, charge coupled device (CCD) camera along with a phase-stabilized local oscillator (LO), also known as the Reference (Ref) pulse. The camera is an Andor iDus 420 with an active detection region measuring 1024 x 255 pixels, in and out of the dispersed spectral plane, respectively. The out of plane dimension is vertically binned and integrated to determine spectral amplitude whereas the in plane dimension is used to collect different wavelengths. The detector is sensitive to radiation in the VIS/NIR range, having a maximum quantum efficiency above $90 \%$ over a wavelength region of $700-900 \mathrm{~nm}$. With this diffraction grating and detector, the spectral field of view is $20 \mathrm{~nm}$ (36 
meV). A time delay of several picoseconds, accomplished with a linear stage mounted retroreflector, between the LO and signal produces spectral interference fringes that allow the complex spectrum to be extracted. The LO is routed around the sample to avoid unwanted preexcitation or distortions of the pulse itself before combining with the FWM signal at a beamsplitter, see Figure 18, Figure 22 (c), and Figure 25. Spectral interferograms are collected as a function of delay time $\tau$, which is stepped in precise phase-controlled increments to generate a series of measurements of delay time versus the emission photon energy.

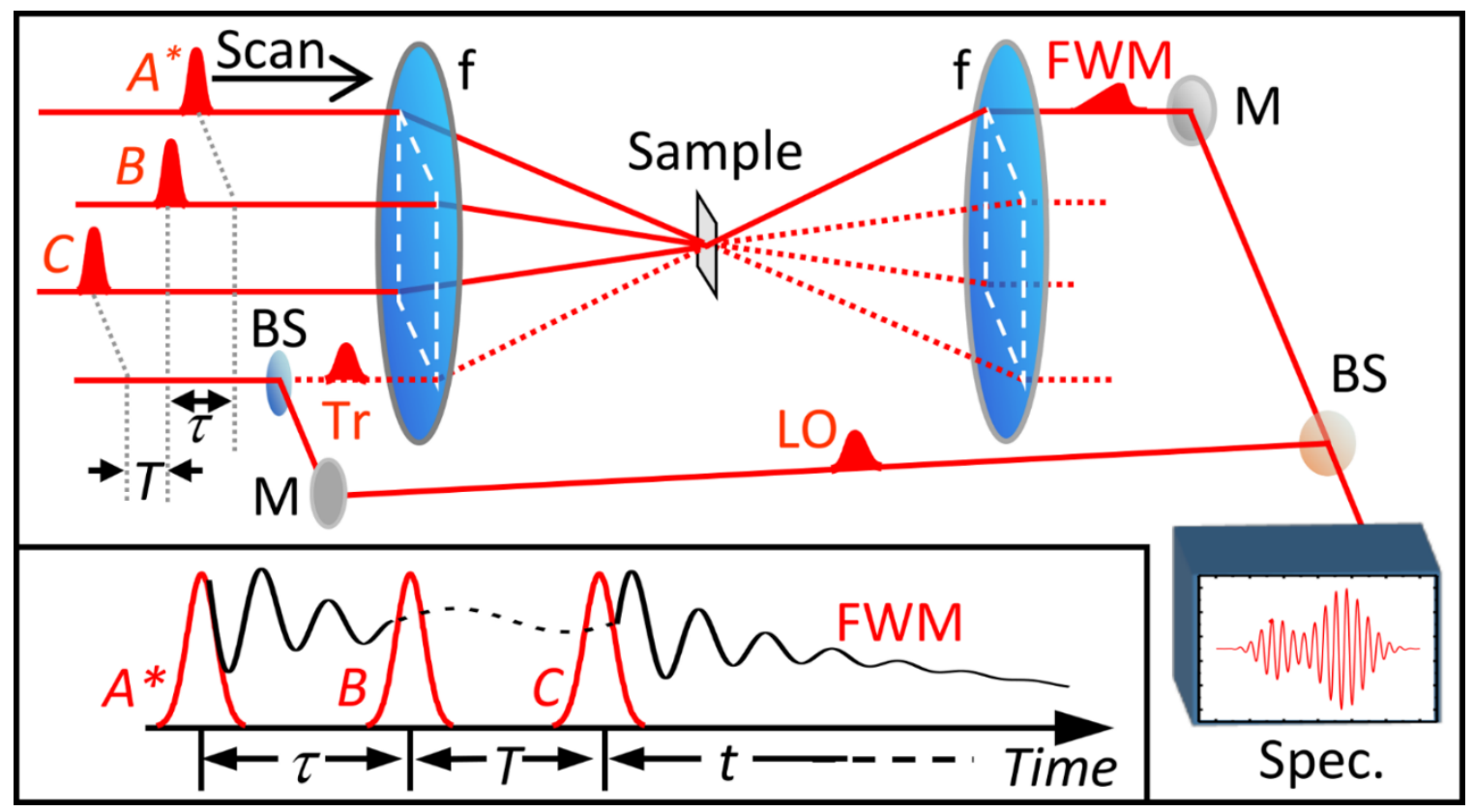

Figure 18 Experimental setup for the two-dimensional Fourier-transform spectroscopy with the sample at the focus. $(\mathrm{f}=$ lens, $\mathrm{M}=$ mirror, $\mathrm{BS}=$ beam splitter, $\mathrm{FWM}=$ four-wave-mixing signal, $\tau=$ period between pulses $A^{*}$ and $B, T=$ period between pulses $B$ and $C$, and $t=$ period after pulse $C$ triggers the emission, $\mathrm{Tr}=$ tracer beam, traces the phase-matched direction, $\mathrm{LO}=$ local oscillator/reference for spectral interferometry, and Spec is the imaging spectrometer with CCD). The inset shows the rephasing excitation pulse sequence.

\subsubsection{Precision Control}

In order for the apparatus to work, precision control of the time delays (both stepping and maintaining) must be possible. There are numerous references of mechanical noise which can vibrate and warp the setup changing optical path lengths to cause the relative time delays to fluctuate. Common examples are air currents and building vibrations caused by human presence such as walking, talking, opening doors, traffic, heating ventilation and air conditioning. 
Furthermore, mechanical precision of the stages limits their spatial resolution which introduces errors during all phases of movement; starting, moving, stopping, and holding. Active and passive stabilization is employed to achieve nanometer stability.

Passive stabilization uses methods which do not specifically monitor and respond to an error signal to actively minimize it, but rather relay on inherent design properties and configurations that are naturally more stable. The optical table itself "floats" on legs supporting the table with pneumatic pistons to minimize vibrations and this is common to many optical setups. A homemade Plexiglas enclosure encapsulates the table during experiments to minimize air currents inside. The optomechanics themselves have increased inertial mass (more massive) and rigidly attached to the table or MONSTR to dampen vibrations and ideally try to make surviving fluctuations common-mode such that relative path length changes minimize. The MONSTR itself is milled from two large blocks of cast aluminum, see Figure 20 and Figure 21. Short, homemade optical mounts are constructed for within the MONSTR. They attach with larger surface area contact than commercial ones. Externally, one or more optics are mounted on large aluminum blocks. When possible, two or more beams will use the same optical element, which effectively guarantees any vibration is common-mode and relative fluctuations are minimized. The Aerotek linear stages responsible for varying timing are themselves high precision and capable of moving and holding a position to within $\sim \pm 7 \mathrm{~nm}$ when their analog and digital PID feedback loops are properly tuned and the stages moved slowly $(\sim 1 \mu m / s)$ and no significant mechanical noise is perturbing them. However, these measures alone are not enough to lower the noise to a few nanometers of fluctuation.

Active stabilization is the final and most challenging layer of noise control. To achieve this, a way to measure the amount of error ( $\mathrm{nm}$ to microns) and correct that error is necessary. A logical choice is using interferometry, which is sensitive to the length scales involved. Thus Michelson interferometers, each with one end mirror mounted to a piezoelectric motor achieve the required $\lambda /(100-400)$ optical path length stability. Piezoelectric motors are a natural choice because they are driven by voltage applied to a crystal (rather than traditional gears) which then changes shape depending on the voltage. Consequently, they can respond quickly 
and with little to no error when changing directions (i.e. no "slop"). There are three interferometers, as depicted in Figure 19, nested inside the MONSTR and one external to relock the reference to beam $C$ after the reference is split off from the primary beam exciting the MONSTR, see Figure 25. Homemade, single-channel silicon photo diode detectors monitor the interference, see Figure 24. Homemade, high-speed analog loop filters filter the measured error signal from the interferometers. High frequency resonances exist in the PZT motor, and thus set the upper frequency limit ( $3 \mathrm{kHz}$ ) for noise that can be cancelled. Analog proportional, integral, and derivative, circuits determine the correct voltage to be applied to the motor to best minimize observed noise. High-voltage drivers then amplify the driving signal and pass it onto the motors. The loop filters and PZT drivers are shown in Figure 28, along with other control electronics such as a dither signal generator used for error signal and PZT motor calibration.

Interferometry is also a reliable method to measure the actual distance a stage travels. By monitoring the interference as the stage moves, the error distance can be calculated and added or subtracted to subsequent steps. Also important to note is that the interferometers are achieved using a co-propagating helium-neon laser beam, and thus the observed interference accurately reflects changes happening to the Ti:sapph laser pulses which will excite the sample. Figure 23 shows the dichroic mirror (DCM) that allows the Ti:sapph and HeNe laser to be spatially overlapped before input into the MONSTR. The HeNe laser is subsequently removed from the output by another DCM which also serves as the second end-mirror for all of the three MONSTR Michelson interferometers. A long pass filter eliminates any HeNe light which manages to pass through the non-perfect DCM. An external interferometer relocks Ref to C outside of the MONSTR and uses the Ti:sapph laser pulses themselves for this stabilization, see Figure 25. 


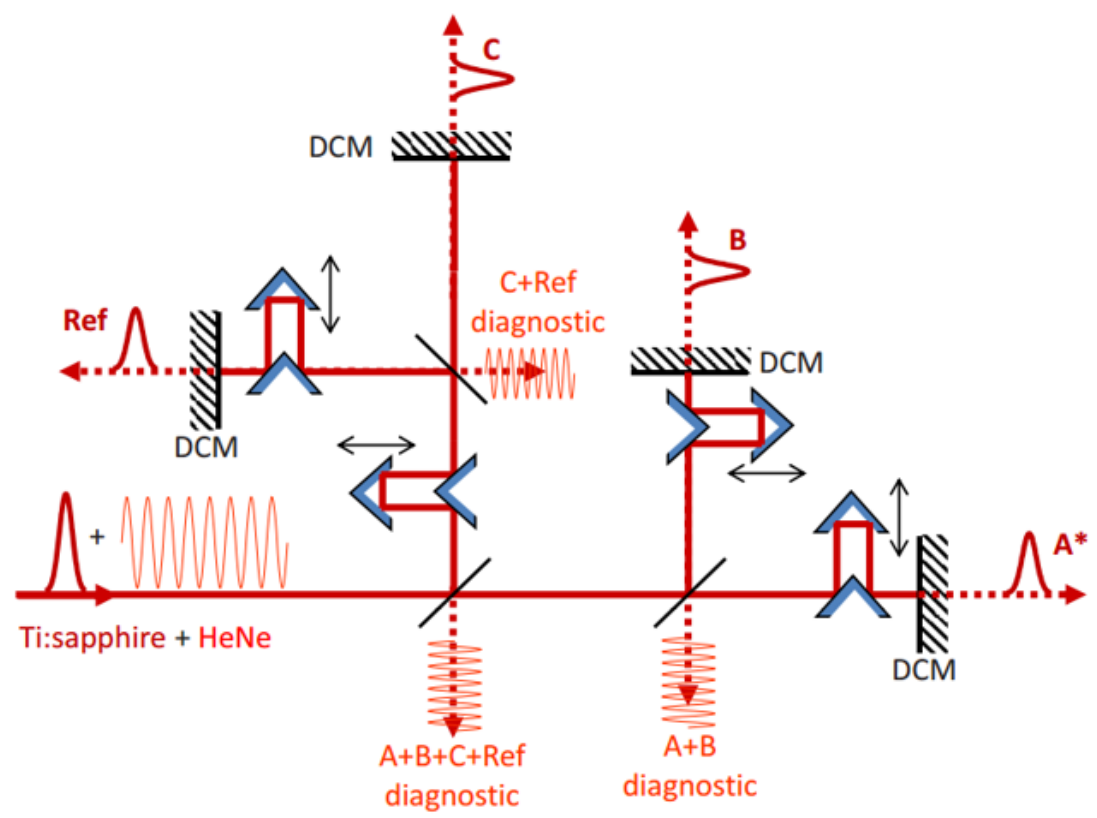

Figure 19 Schematic representation of the nested Michelson interferometer layout. $A^{*}, B, C$, and Ref are the Ti:sapphire laser pulses used to perform the 2DFT experiment. The HeNe laser beam is reflected from the DCM to provide diagnostic information for the interferometers. The proposed layout is folded such that the DCM is a common optical element. Reproduced from reference [53].

\subsubsection{Phase Retrieval}

An advantage of our MDCS scheme is that because the signal is detected using spectral interferograms resulting from a heterodyned signal, the technique is phase-sensitive and able to extract the real part of the emitted spectrum rather than just the amplitude. This real part of the spectrum contains more information about the microscopic physics that determine the systems evolution.

To accomplish this, an algorithm to determine the initial signal phase and track its evolution was developed by Bristow et al [54] for the MONSTR setup and will be summarized here. The signal phase is tracked with respect to the reference pulse. Therefore, determining the initial phase with respect to the reference as well as the initial phase of the exciting beams must be done before each scan and in such a way as to leave the system ready to scan at the end of the procedure. This means that all interferometers are locked. Any auxiliary measurements which 
my interrupt or physically disturb the path lengths (by more than a few hundred nanometers) would not accurately reflect the in situ phases during an experiment.

A replica focus of the sample focus has been created using a clear window, 50x microscope objective, and imaging CCD Mightex camera, see Figure 25. It serves the dual purpose of alignment as well as extracting the phase information from the exciting laser beams.

The replica focus provides temporal and spatial information about pulses A, B, C and Ref. Spatially, all four beams should be focused at the same spot and should maintain a square box as the microscope objective is panned through the focus. This information allows for fine beam alignment. Temporally, interference is only visible, and maximized, when the pulses are overlapped. Therefore, maximizing the interference is a method to find zero relative time delay (autocorrelation) between two pulses. The orientation of the interference fringes also supplies more information about the spatial beam arrangement. The autocorrelation procedure is detailed in appendix A.5 Autocorrelation.

The particular embodiment for this setup of the phase acquisition and processing procedure is covered in appendices A.7 2D Data Acquisition Procedure, with Phase Retrieval and A.8 Processing Phase Retrieval Data, respectively. Pre-scan interference patterns utilizing interference at the replica focus and spectral interferograms at the spectrometer capture the initial phases of the exciting beams and reference, denoted $\phi_{C A M}$ and $\phi_{T R}$, respectively. "CAM" communicates the phase is determined from interference patterns captured on the replica focus camera and "TR" communicates it is determined from interferograms between the tracer and the reference recorded at the spectrometer. These are related to the spectral interferogram of the signal and reference simply as [54]:

$$
S_{I, S I}(\omega)=\hat{\chi}^{(3)}\left(\omega ; \omega_{A} \omega_{B} \omega_{C}\right)|\tilde{E}(\omega)|^{4} e^{i\left(\phi_{S R}-\phi_{C A M}-\phi_{T R}\right)}
$$

The final required term is $\phi_{S R}$, the signal phase, must be hand-picked by optimizing the measured phase (the offset should be small, and the phase flat except for near resonances). It is consequently the greatest systematic reference of error aside from phase fluctuations that occur after the pre-scan recordings (and can in principle occur during the scan). 


\subsubsection{Photographs of Actual Implantation}

To further communicate the complexity of the experimental implementation as well as illustrate how the various tasks and requirements are actually accomplished, diagramed photographs are presented of its current completed construction.

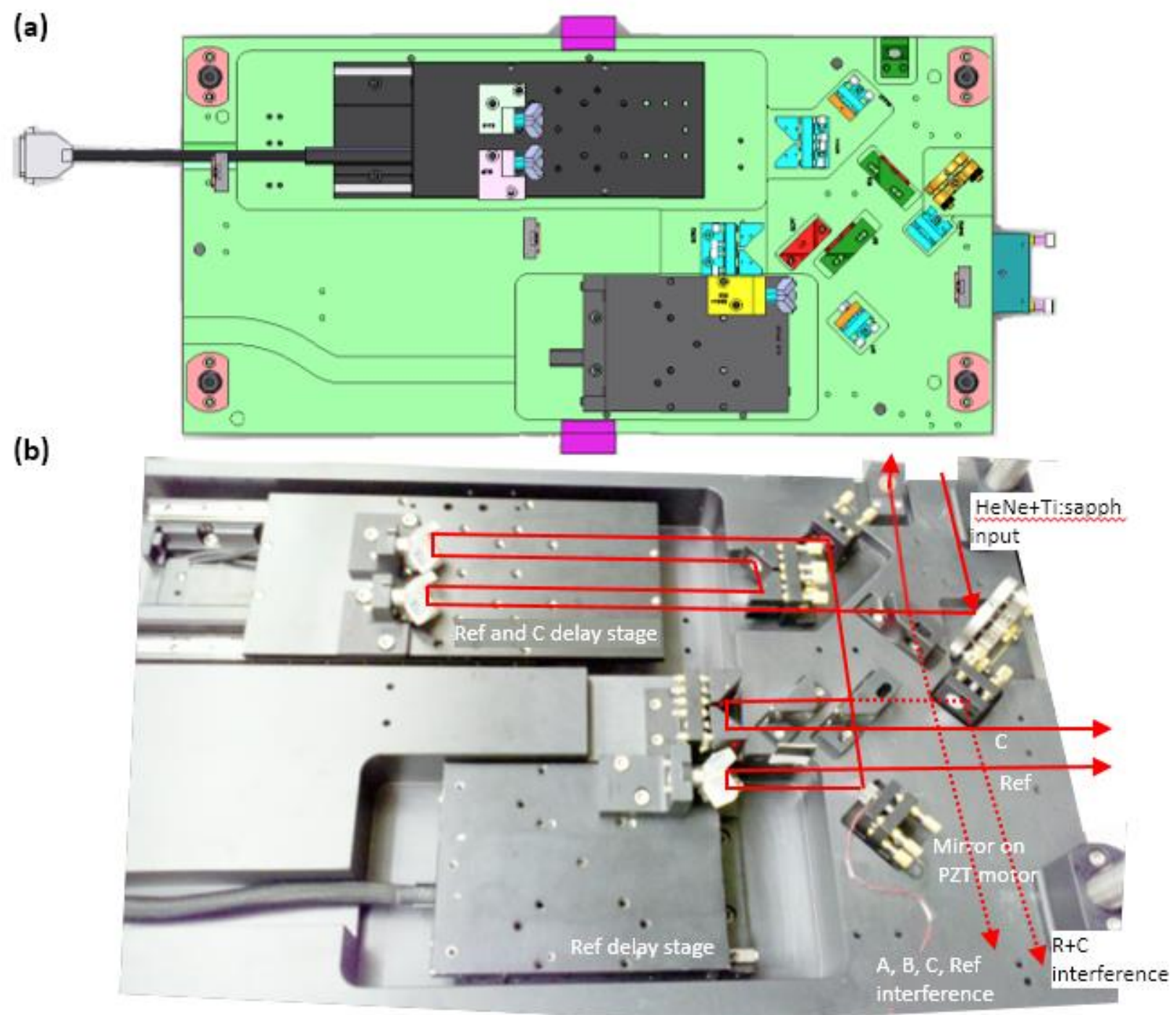

Figure 20 (a) Solid Works design file for the bottom MONSTR deck and (b) a photograph and diagram of the laser paths. 


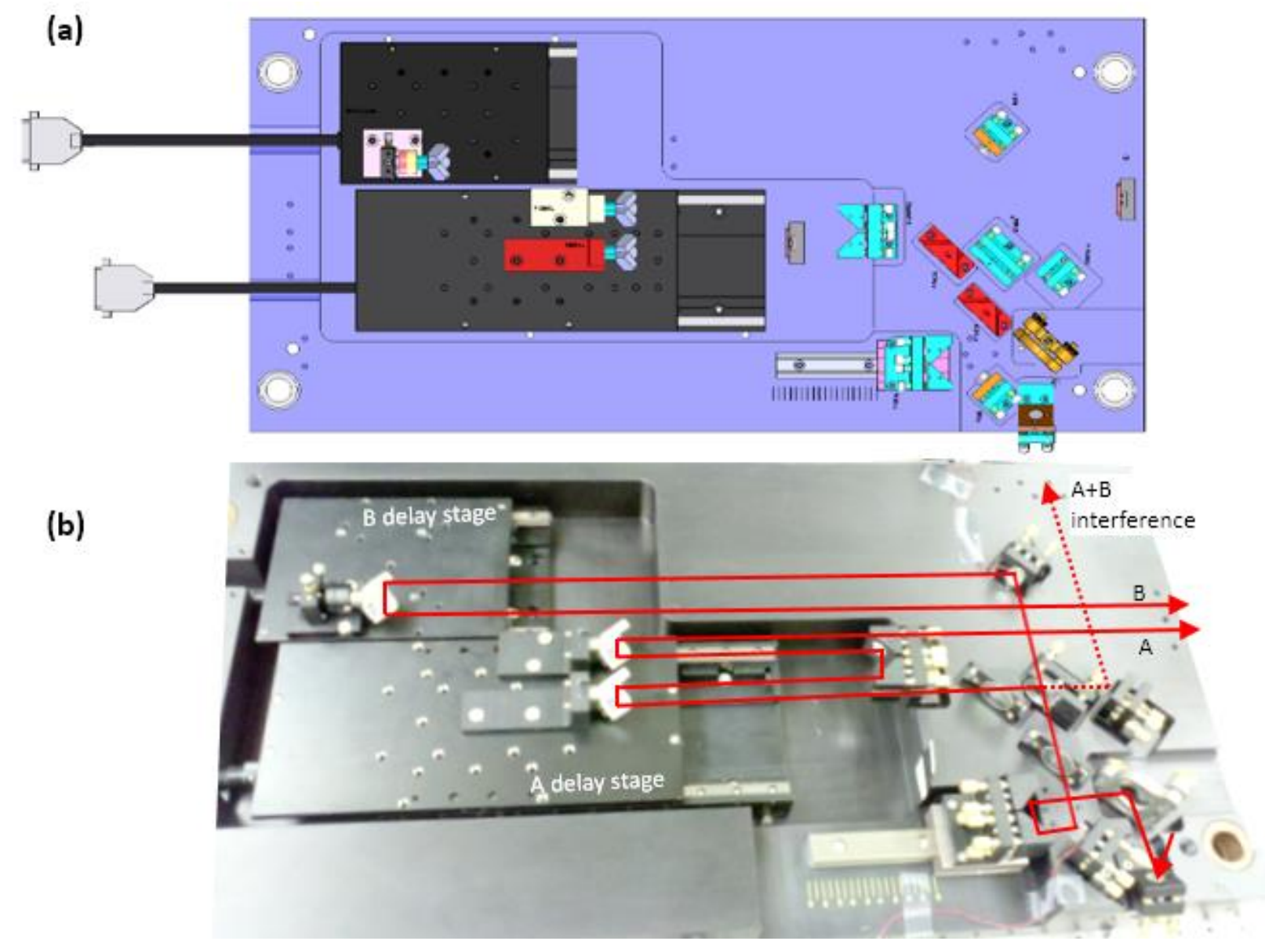

Figure 21 (a) Solid Works design file for the bottom MONSTR deck and (b) a photograph and diagram of the laser paths. 


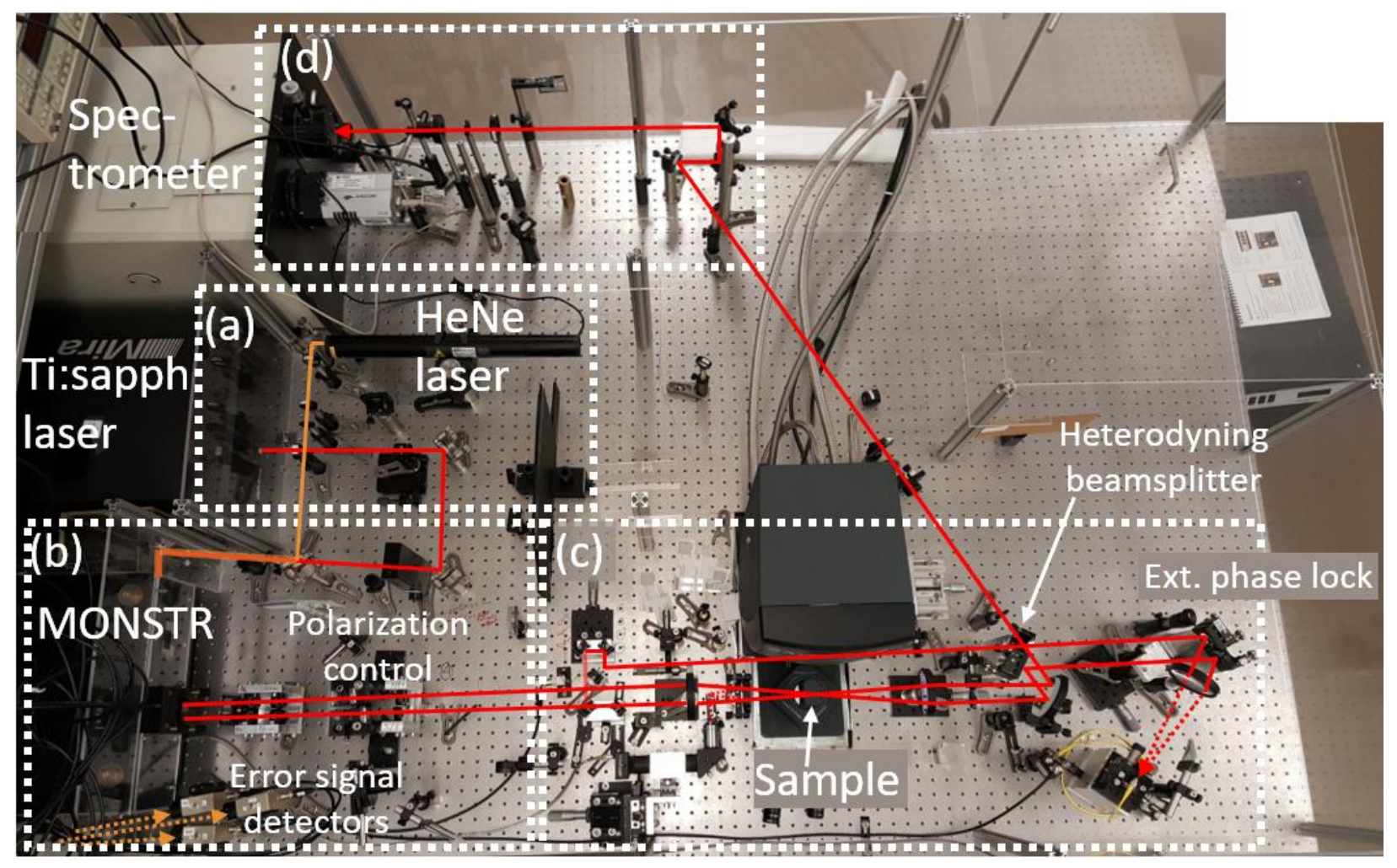

Figure 22 Photograph of MDCS optics, showing (a) the Ti:sapph and HeNe laser references, (b) the MONSTR, error signal detectors, and polarization control (c) the cryostation and sample focus, replica focus, heterodyning, and external interferometer to phase lock the reference to $C$. HeNe beams are orange and Ti:sapph beams in red. 


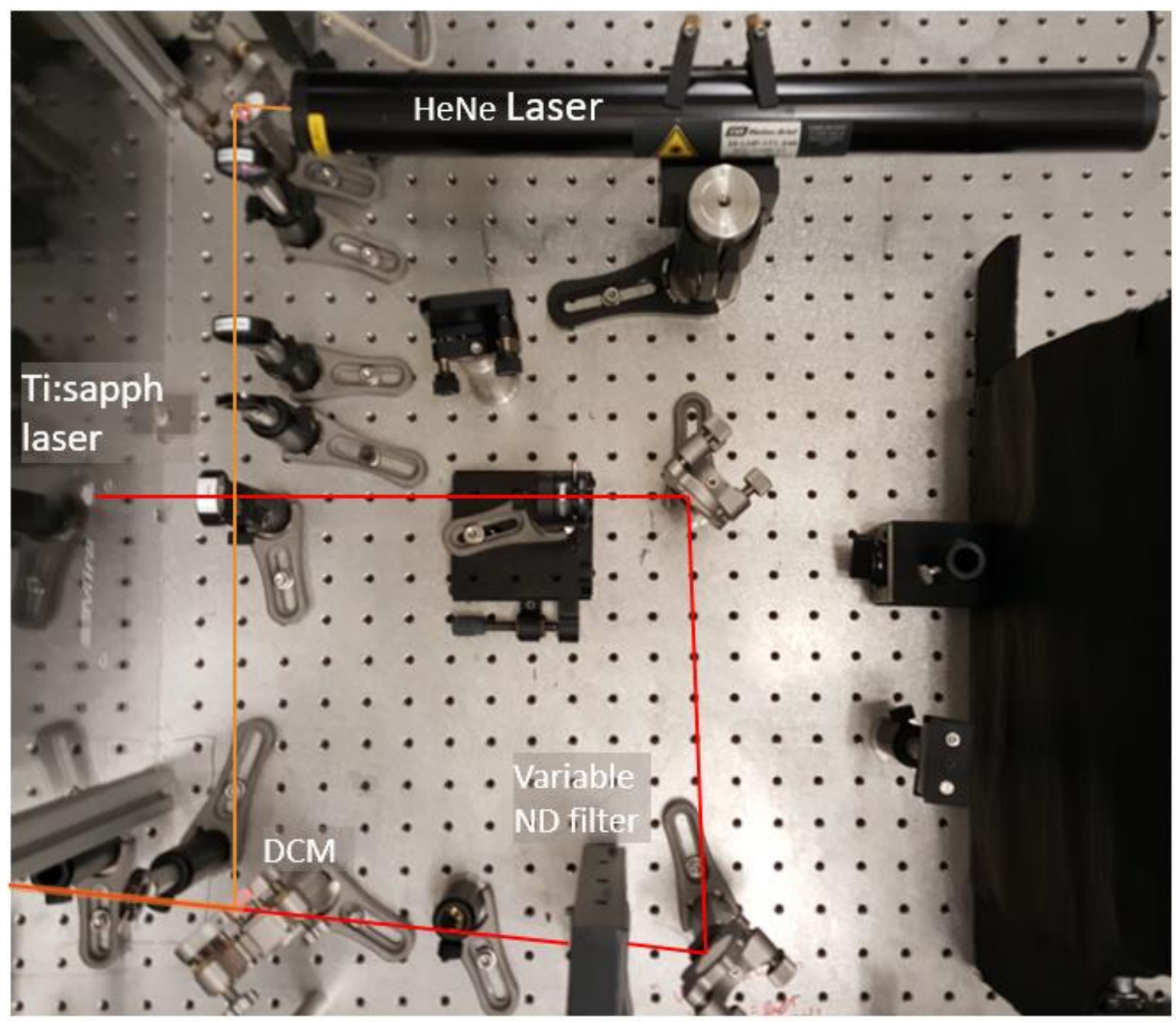

Figure 23 MDCS input lasers, a closer view of Figure 22 (a) showing the laser references, variable neutral density (ND) filter, and a dichroic mirror (DCM) combining the two beams collinearly. 


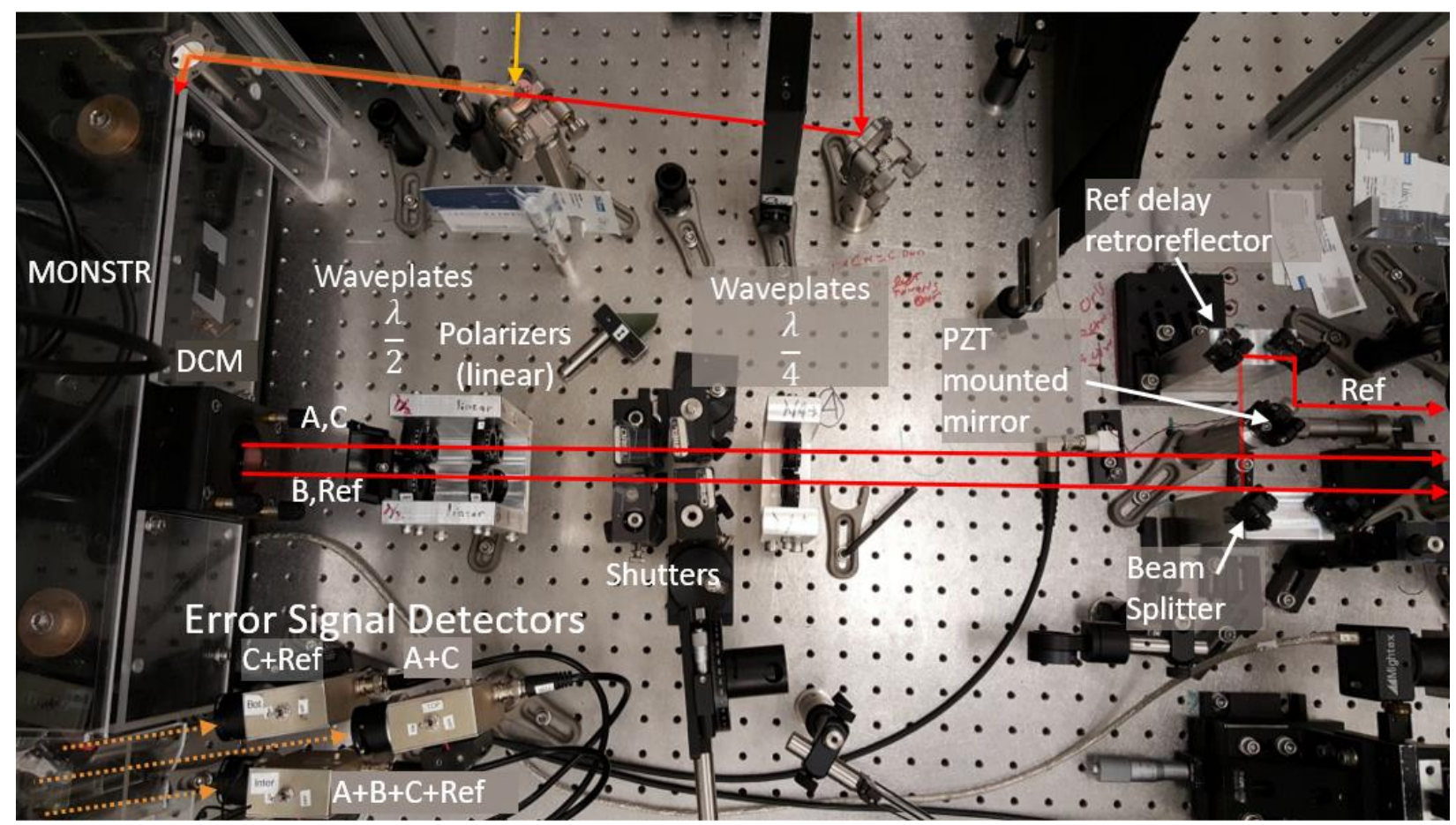

Figure 24 MONSTR output, polarization control, and error signal detections, closer view of Figure 22(b).

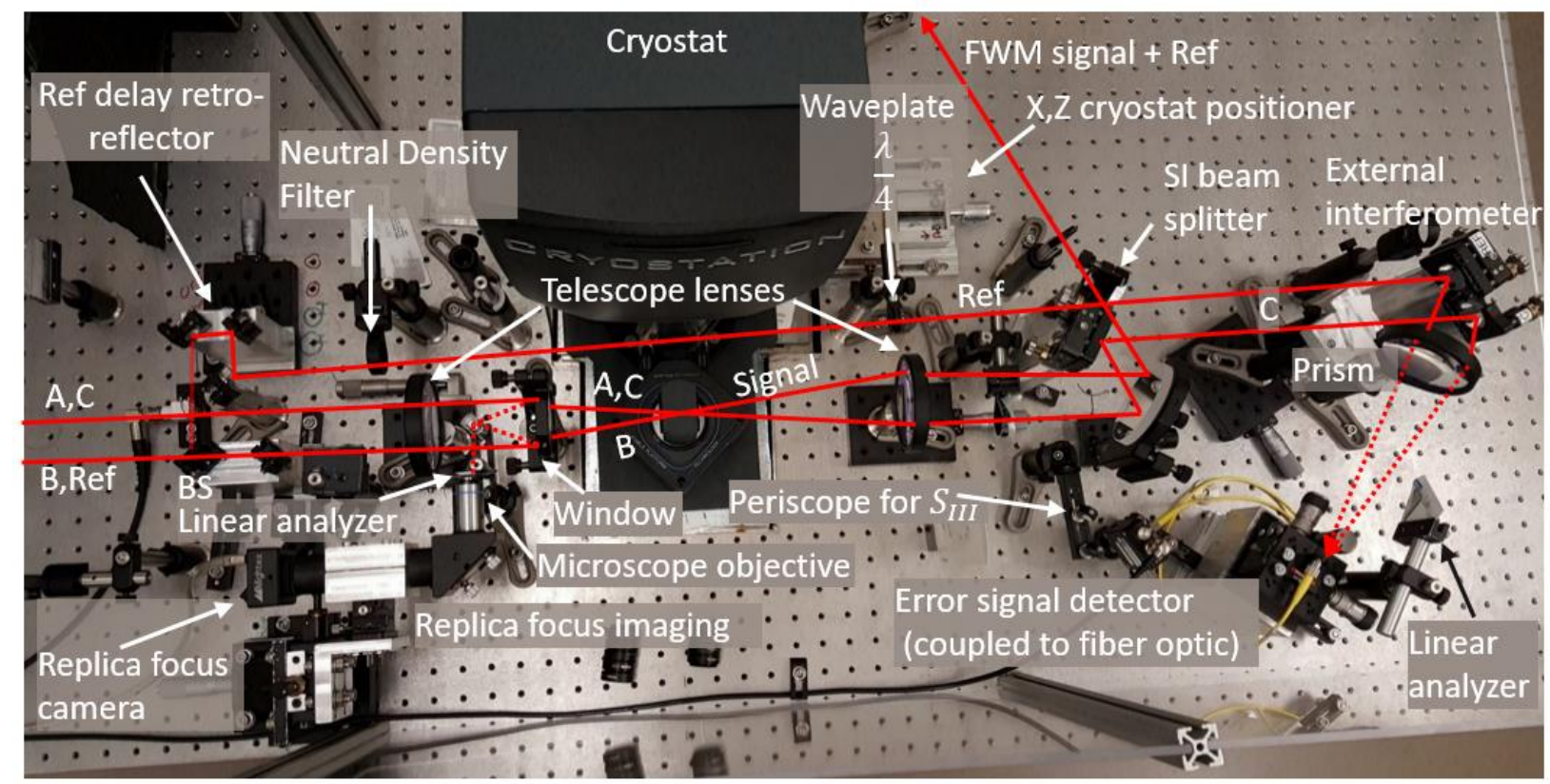

Figure 25 Replica focus, sample focus, external interferometer, and signal + reference combination, a closer view from Figure 22(c) 


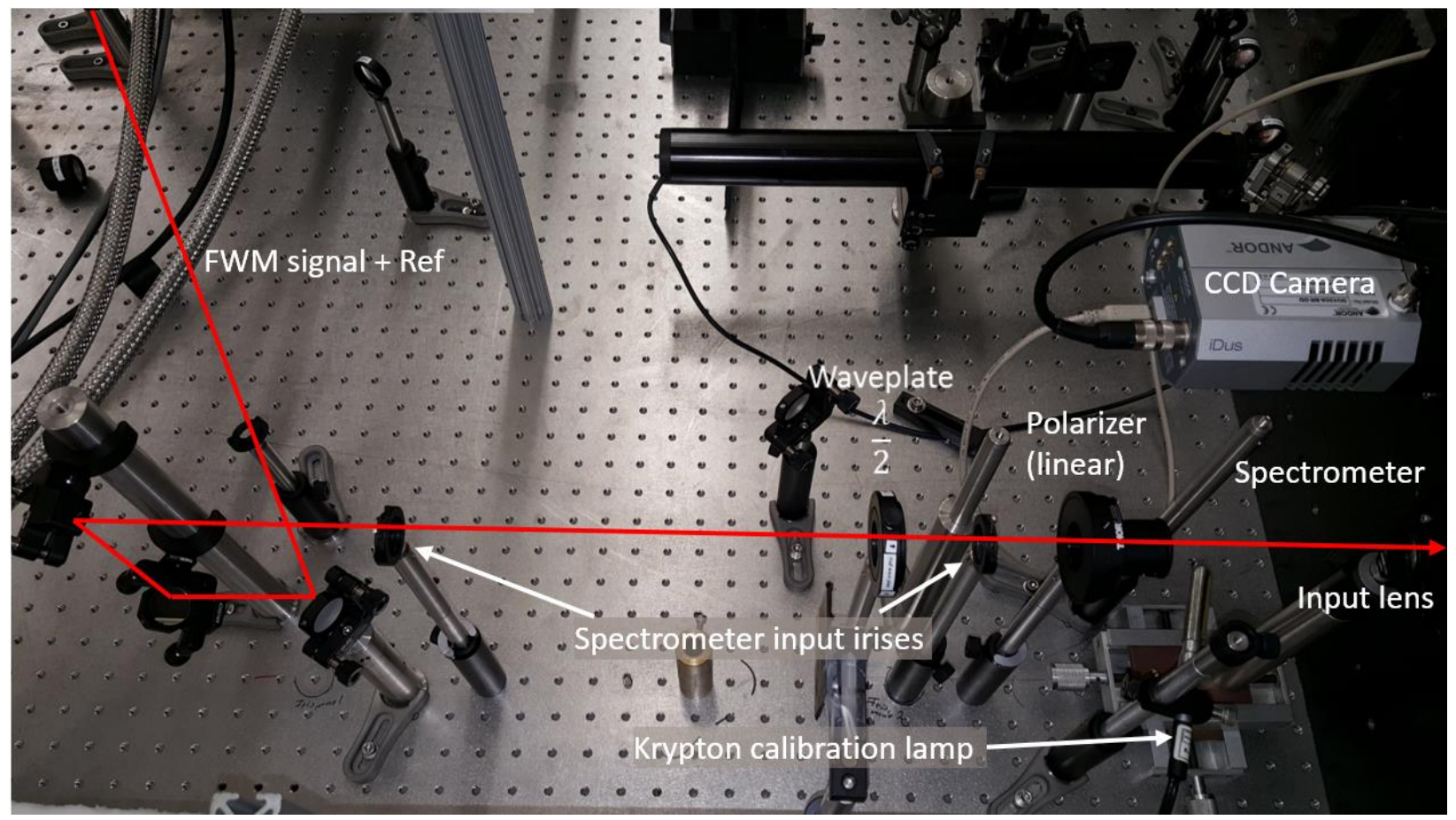

Figure 26 Detections optics including polarization control as well as calibration lamp (not in use), a closer view Figure 22 (d).

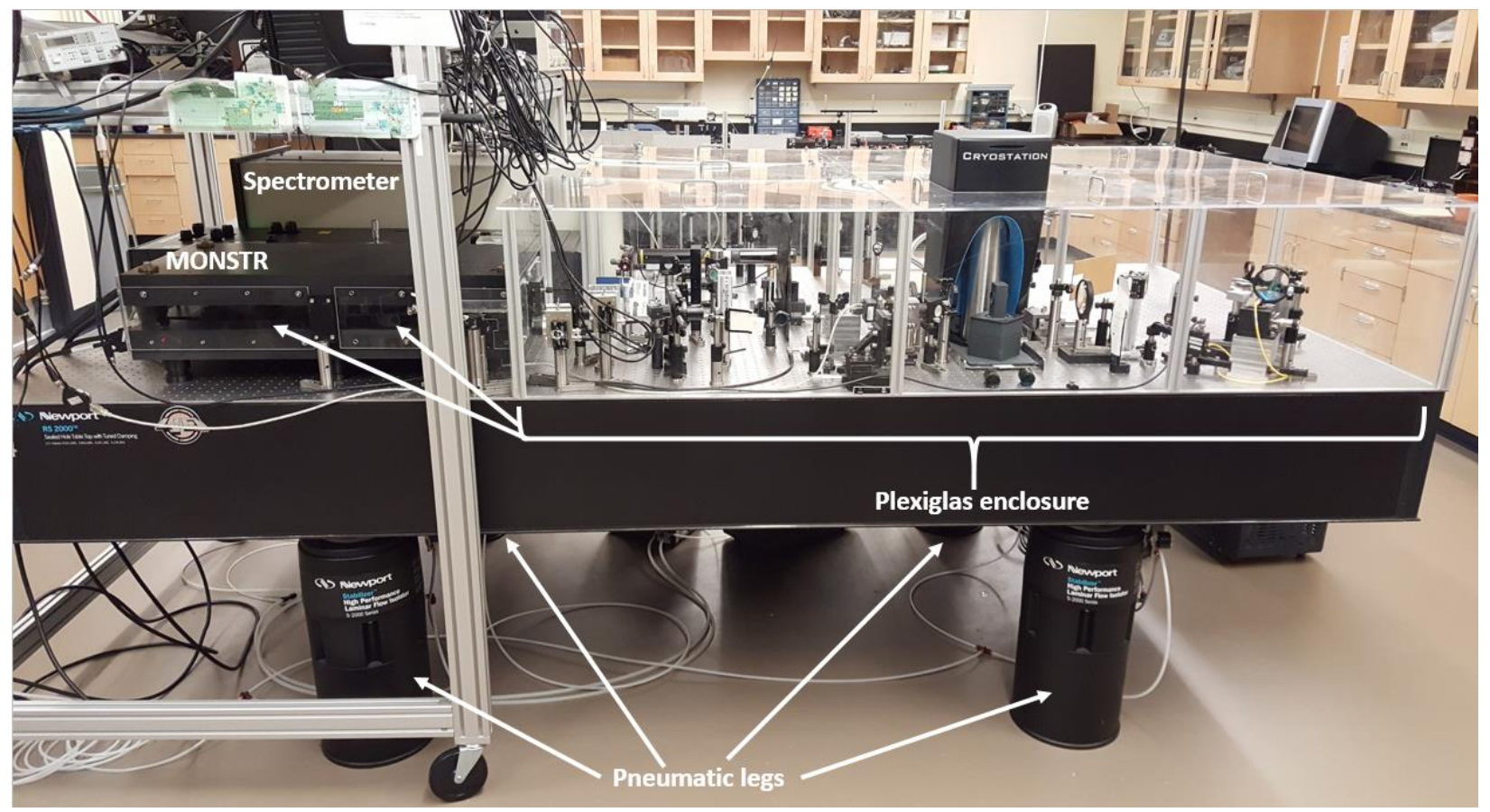

Figure 27 Side view of the setup showing the Plexiglas enclosure and pneumatic table legs for phase stabilization. 


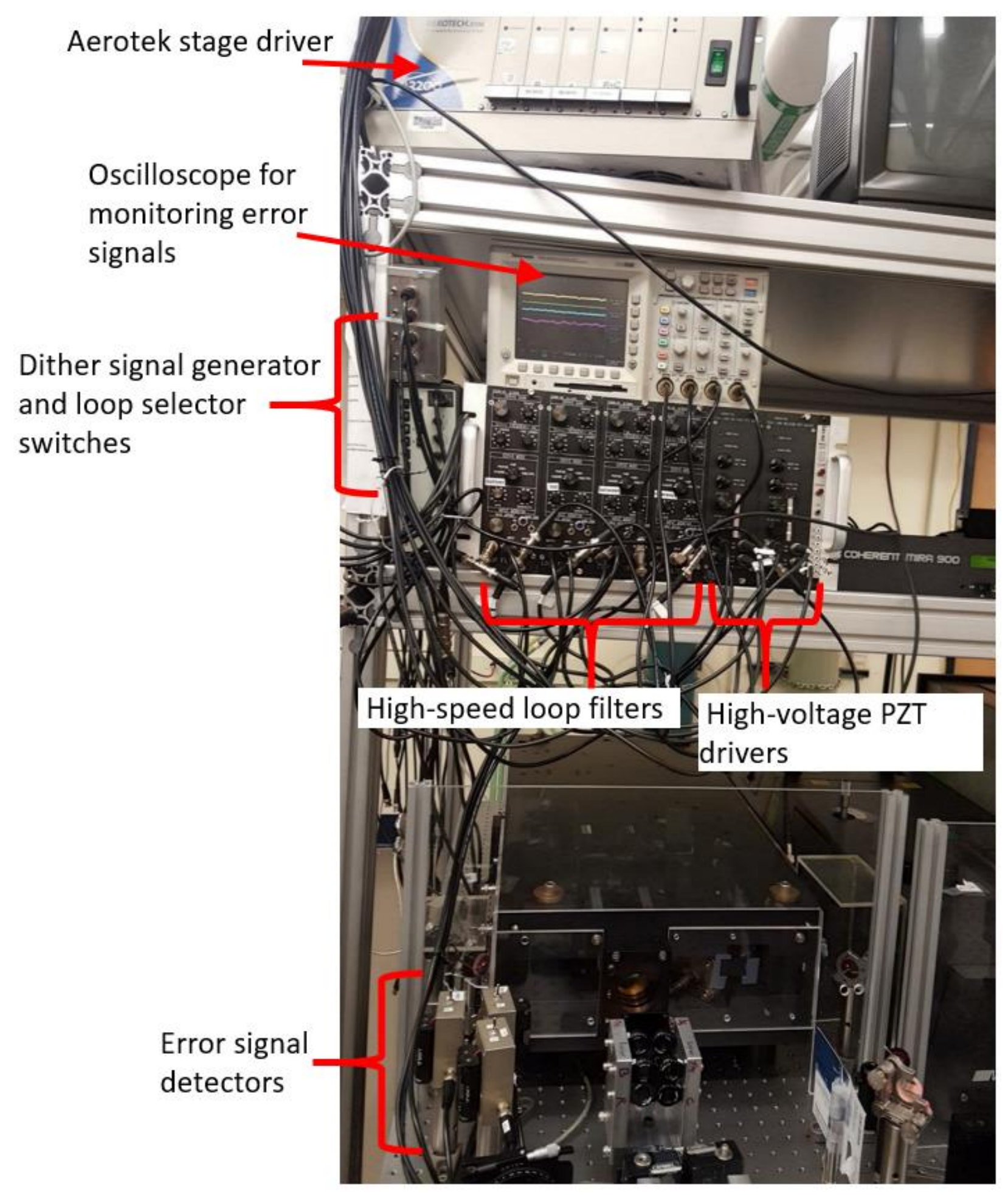

Figure 28 Error signal monitor and control electronics, desktop computer not shown. 


\subsection{Phase Cycling}

A type of noise cancellation exists for coherent, phase sensitive spectroscopies that does not for other types called phase cycling. Because the signal is sensitive to the phase of the pump pulses, however the noise is incoherent and thus not phase sensitive, one can discriminate between the two by varying the pump phase by a fixed amount and observing the change (or lack thereof) in the detected data. The phase is typically cycled by an odd-integer multiple of $\pi$ such that phase shift swings the signal from some positive value to exactly that same value but negative. The two datasets can then be subtracted and halved to remove the noise. This operation is performed for every data point, and in the current work this occurs at each time step. The phase is cycled rather than constantly incremented in one direction so as to not accrue a phase shift (and inadvertently introduce another varied time period) in the signal additional to that caused by the time delay being purposefully varied. Figure X (a) shows the results of a MDCS, rephasing scan done without and (b) with phase cycling.

\subsection{Absorption Spectroscopy}

Absorption spectroscopy is a linear technique which provides significant amount of useful information while requiring considerably less work compared to nonlinear spectroscopies. Absorbance, related to the material properties by Beer's law, is defined as:

$$
A=\log \frac{I_{i}}{I_{t}}=\alpha d=\varepsilon c d
$$

where $I_{i}$ and $I_{t}$ are the incident and transmitted light intensities, respectively. Though only absorbance data is present in this work, the absorbance $A$ can be further decomposed into the product of the absorption coefficient $\alpha$ and sample thickness $d$. Beer's law allows $\alpha$ to be further decomposed into the product of the molar attenuation coefficient $\varepsilon$ and the material's concentration $c$.

A complication exists when the samples are attached to a substrate (as is the present case), because the intensities recorded are not the intensities required to directly calculate the absorbance, namely the incident and transmitted intensity of just the sample. However, with 
knowledge of the index of refraction of each material and assuming negligible scatter in both materials, the intensity of light entering the sample, and leaving the sample can be extracted. Figure 29 shows a diagram of the sample and substrate, as well as the intensities important for extracting the absorbance. The index of refraction of the sapphire $\left(\mathrm{Al}_{2} \mathrm{O}_{3}\right)$ substrate $\mathrm{n}_{1}$, GaAs sample $n_{2}$, and air $n_{3}$, are $1.762,3.35[128,129]$, and 1.0002776 at $800 \mathrm{~nm}$, and at low temperature for the crystals, respectively. $I_{0}$ (unknown) is the intensity of the light incident at the substrate-sample interface. $I_{1}$ is the measured intensity of light leaving the sapphire substrate. $I_{2}$ is the measured intensity of light leaving the entire structure. $\mathrm{I}_{\mathrm{i}}$ (unknown) is the desired intensity of light entering the sample. It (unknown) is the desired intensity of light leaving the sample incident on the sample-air interface.

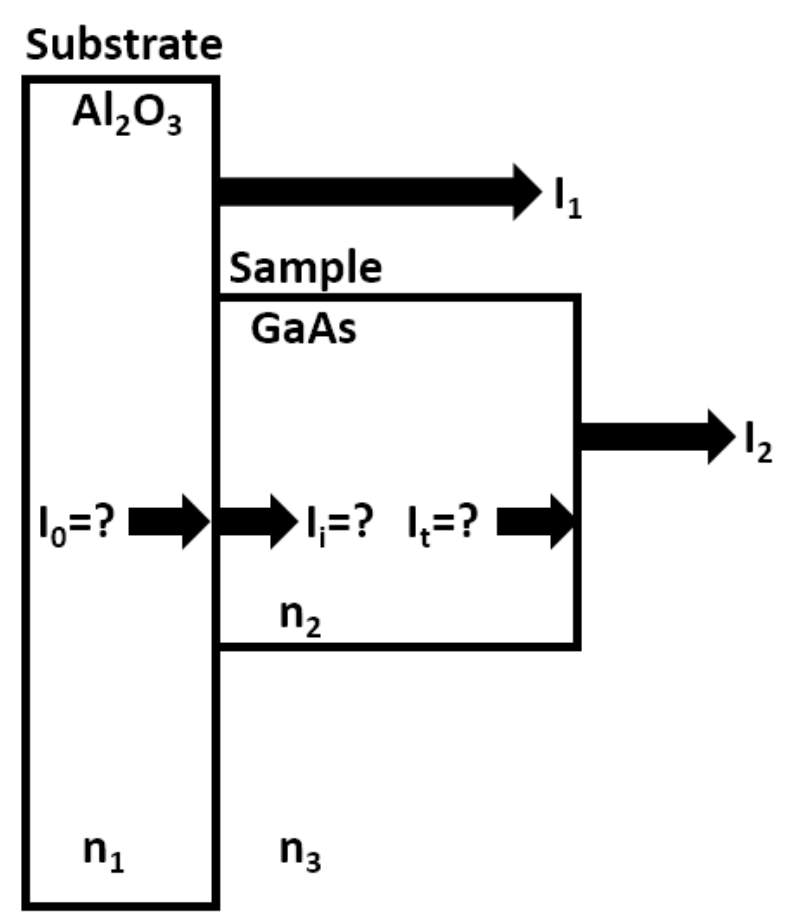

Figure 29 Diagram of sample mounted to substrate and relevant intensities

At a given interface between two materials having indices of refractions $n_{1}$ and $n_{2}$, the reflection and transmission coefficients can be calculated as:

$$
R_{12}=\left(\frac{n_{1}-n_{2}}{n_{1}+n_{2}}\right)^{2}
$$




$$
T_{12}=4 \frac{n_{1} n_{2}}{\left(n_{1}+n_{2}\right)^{2}}
$$

They are normalized, $R+T=1$, assuming normal incidence, and no scatter which would enter as another term. These coefficients communicate how much light is reflected or transmitted from knowledge of the indices of refraction.

We can now solve the above problem:

$$
\begin{gathered}
I_{0}=\frac{I_{1}}{T_{13}} \\
I_{i}=T_{12} I_{0} \\
I_{t}=\frac{I_{2}}{T_{23}}
\end{gathered}
$$

The absorbance can now be computed trivially by taking the negative logarithm of the ratio of the intensities.

$$
A=\log \left(\frac{T_{12} I_{0}}{I_{2} / T_{23}}\right)
$$

Absorbance data in this work is used for determination of the conduction band edge, which is characterized by an increasing absorbance as photon energy increases. It also allows for determination of what transitions are present (within the bandwidth of the laser pulse), their linewidths, and how strong those transitions are absorbing photons. Experimentally, this information also serves as a guide when determining excitation conditions for the nonlinear measurements.

\subsection{Data Acquisition and Processing}

A majority of the equipment is computer controlled and MDCS scans, once setup, are executed by computer programs. As this is not a commercially available system, a considerable amount of LabVIEW and Matlab coding was necessary to make the experiment possible. LabVIEW code, due to its unique graphical programming style, will not be presented here. At the end of a scan, thousands of spectra must be processed to generate a single two-dimensional spectrum. The procedure for processing data is presented in appendix A.7 2D Data Acquisition Procedure, 
with Phase Retrieval and A.8 Processing Phase Retrieval Data and the Matlab code in appendices A.10 A.14 Tracer/SI Phase Code.

During data acquisition, the FTSA code is executed to monitor the scan's progress. Several plots are immediately available to evaluate the quality and status of the scan. The following data is acquired on strained bulk GaAs, presented in detail in Chapter 4 Bulk Gallium Arsenide Excitons

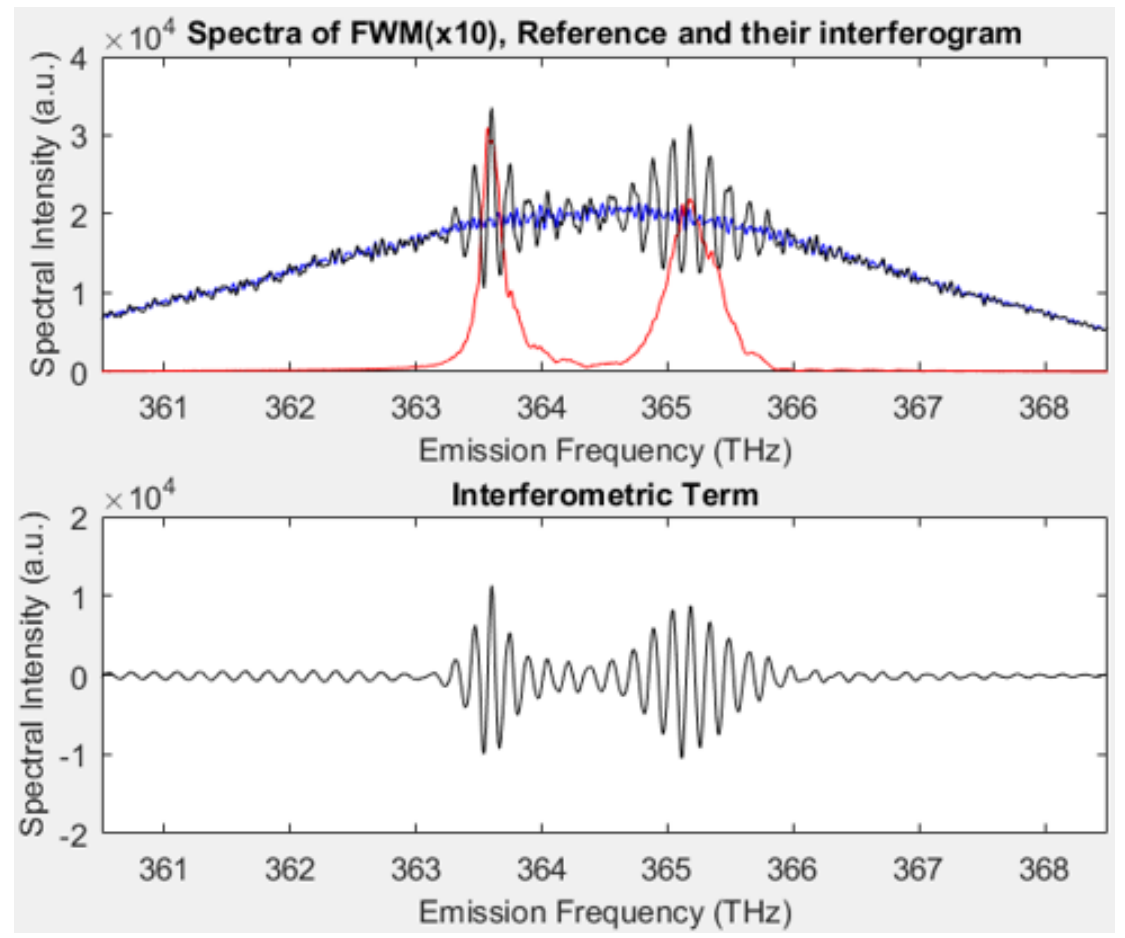

Figure 30 (upper panel) spectral interferogram (black), reference spectrum (blue) and the FWM signal (red) and (lower panel) the extracted interferometric term from the measured spectral interferogram intensity

Figure 30 in the upper panel overlays the reference pulse spectrum, spectral interferogram, and FWM recording before the scan and the extracted interference term. The reference pulse is attenuated such that it is 3-5 times the intensity of the FWM signal and mixes with the signal with a time delay of 5-10 ps (with the reference arriving first). Experience has proven this to result in the largest amplitude of the spectral interference fringes. The frequency of the fringes ( $a$ beat frequency) is determined by this time delay. A zero time delay is not ideal because of the spectral fringes would be low enough frequency to be obscured by the $\propto \frac{1}{f}$ rise 
encountered near zero frequency in Fourier transforms. The lower panel shows the extracted interferometric term, which should be flat except for spectral regions were the FWM signal is present. This current data shows some noise, which is the ripple in the wings. Slight changes to the beamsplitter combining the reference signal can sometimes solve this problem.

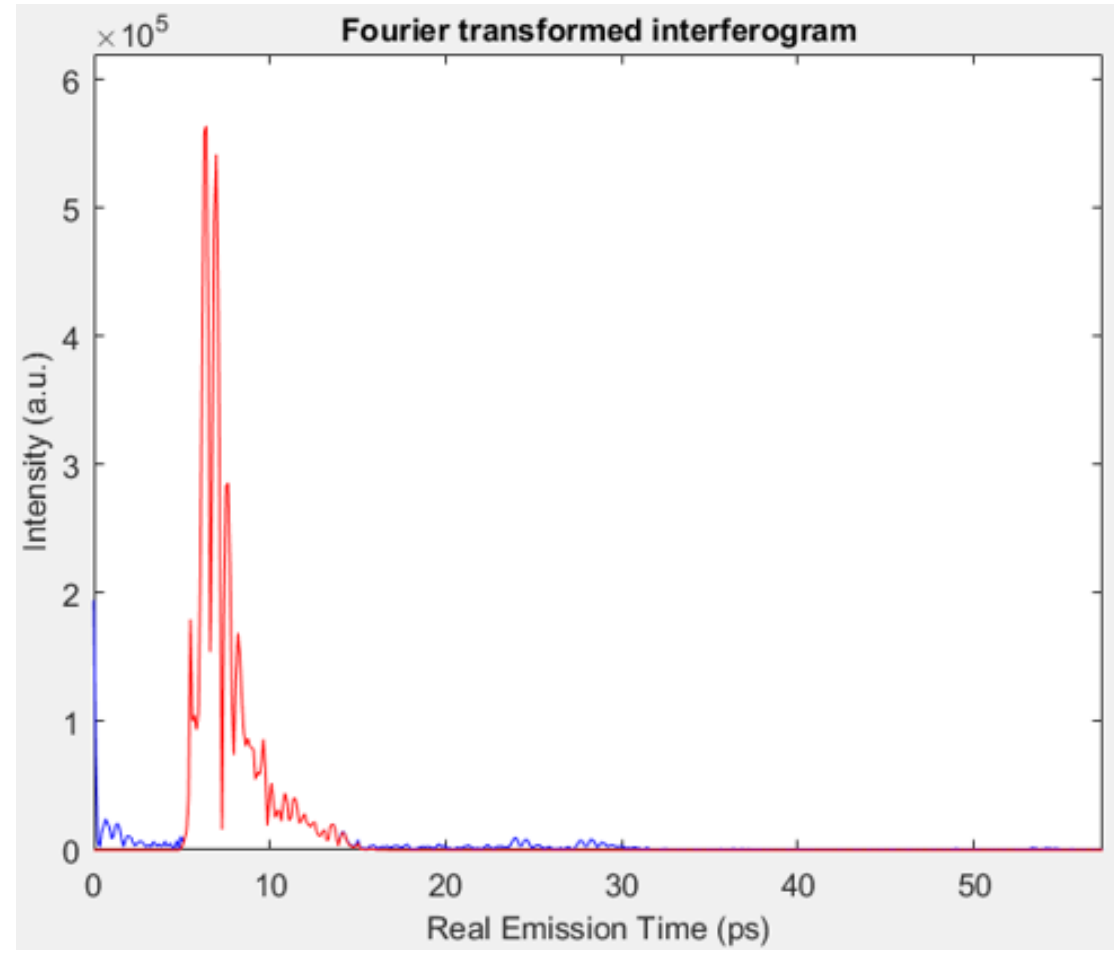

Figure 31 The time domain representation of the FWM + Reference interferogram. The raw data is red and the truncations in blue.

Figure 31 shows the total intensity of the interference between the signal and reference rise and decay in time. This is useful for windowing the data in the time domain. Two cuts, in blue truncate the data before and after the decay of the signal. This reduces noise and streaking in the final $2 \mathrm{D}$ spectrum. 

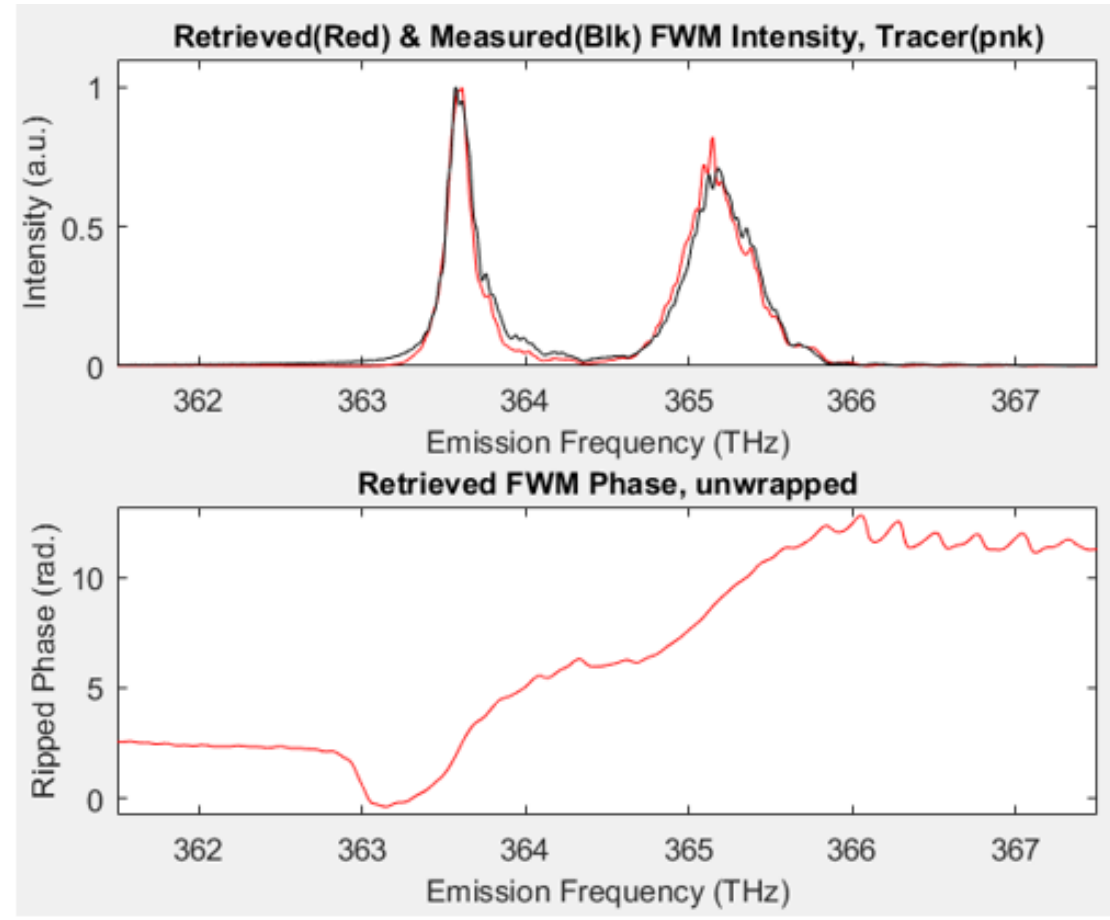

Figure 32 (upper panel) The measured FWM signal spectrum compared to that retrieved from the interferogram and (lower panel) the unwrapped signal phase as a function of frequency

Figure 32 shows the overlay of the pre-scan measured FWM and that extracted from the interferometric term in Figure 31. Depending on the scenario, differences may be good or bad. If the signal to noise ratio is low, the retrieved may overcome some of that noise and provide a better representation than the FWM trace recorded before scanning. However, if low signal strength is not the case, this represents also bad alignment or reference attenuation. 


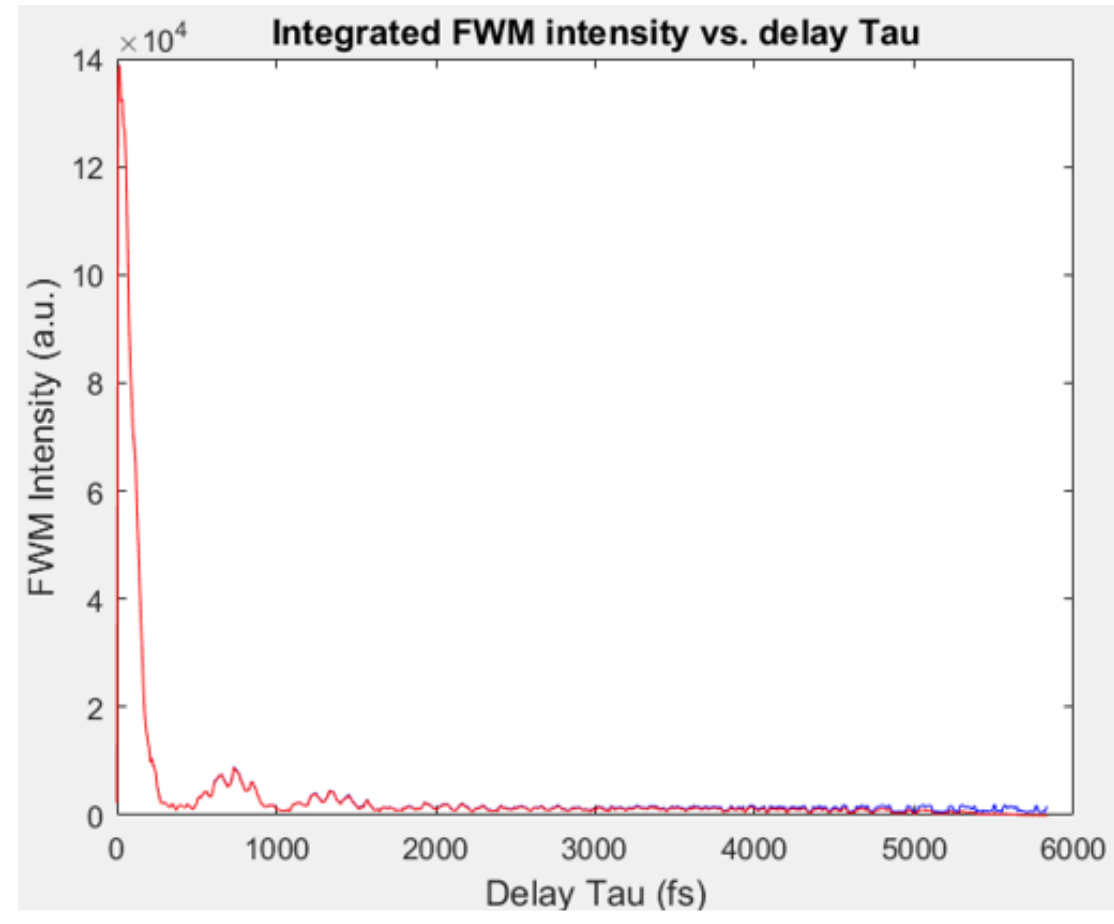

Figure 33 The integrated FWM as a function of the scanned time delay (tau in this case)

One of the most important outputs from FTSA is the FWM intensity as a function of the scanned time delay, see Figure 33. In the 2D experiments conducted in this thesis, only one time delay is scanned and Fourier transformed. The second spectral axis is provided by an optical Fourier transform by the spectrometer. Therefore, from this plot it is possible to learn when a scan is completed. When the signal stops beating (in the case of multiple resonances) and decaying as a function of increasing delay, the scan is finished. From this plot the coherence time can also be estimated as the time it takes for the signal to decay to $1 /$ e of the initial value. 


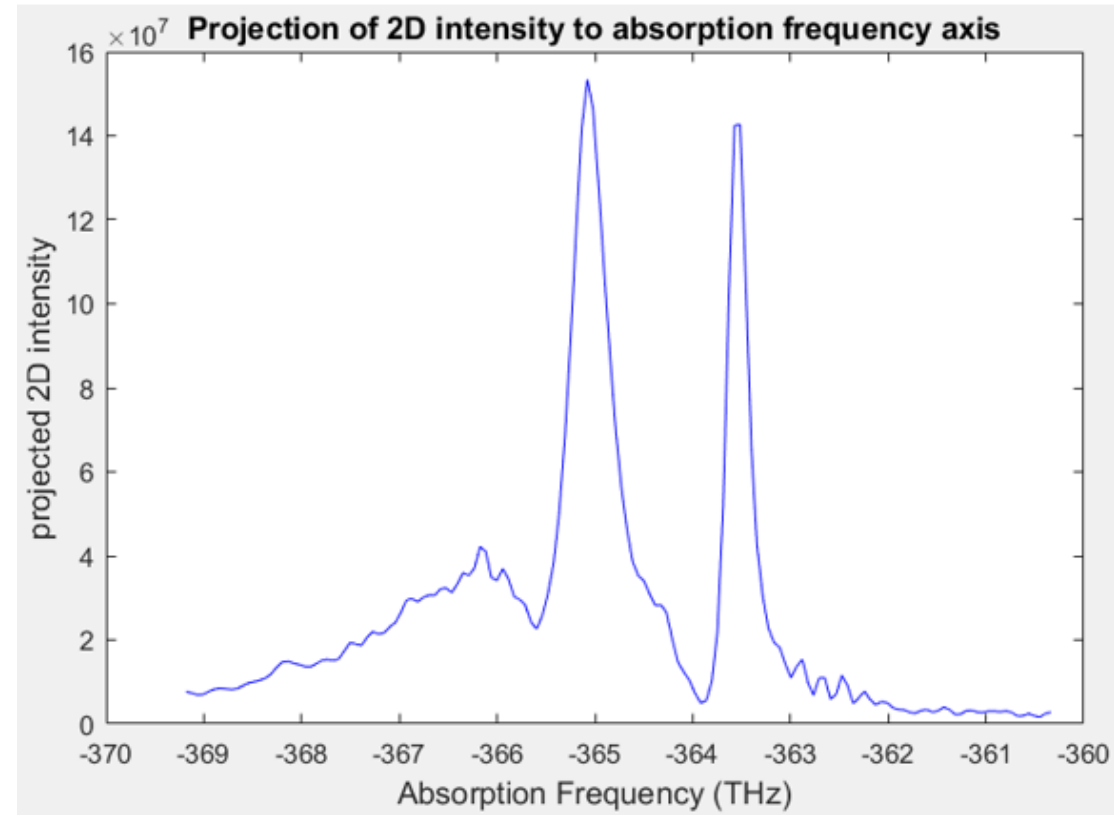

Figure 34 The projection of the 2D spectrum onto the $1 \mathrm{D}$ absorption axis

A projection of the data onto the absorption axis, see Figure 34, provides some of the information supplied in linear absorption data and serves as another connection to more traditional one-dimensional spectroscopies. 


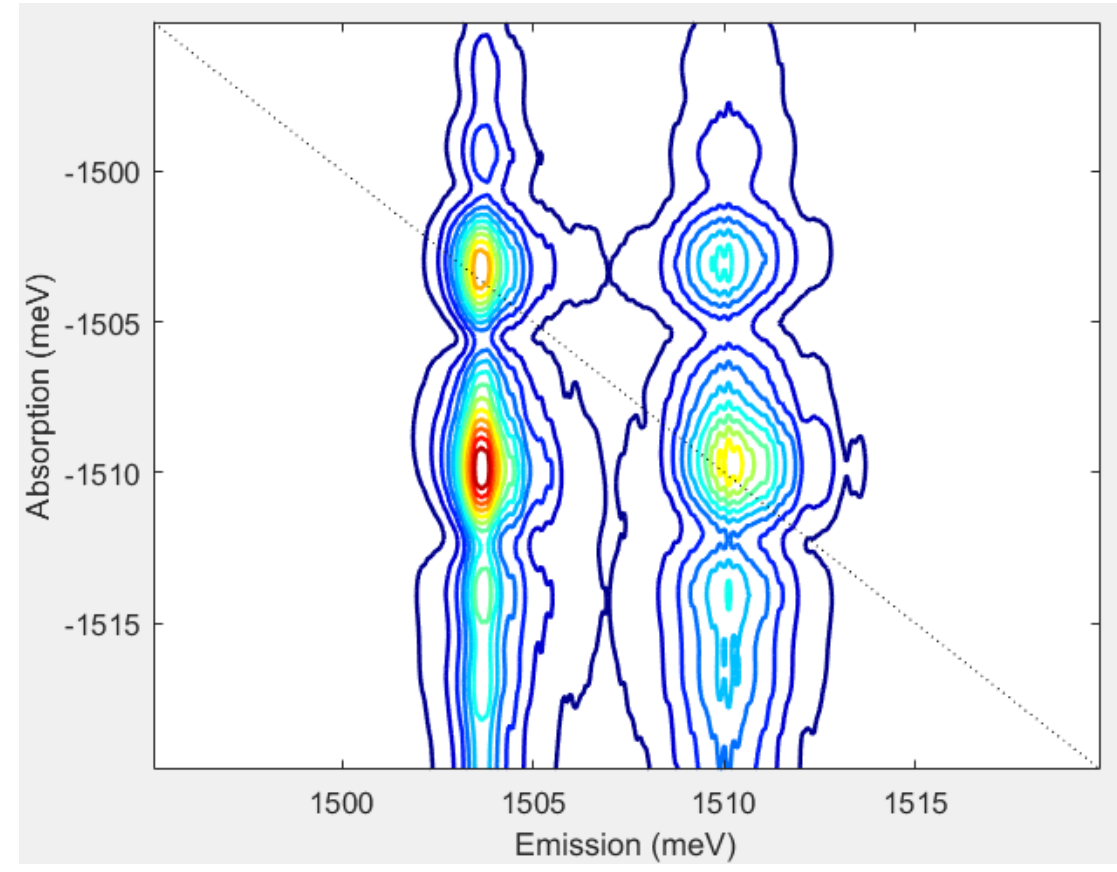

Figure $352 \mathrm{D}$ amplitude spectrum from incomplete scan. notice the overall widening of the peaks, particularly along the absorption axis

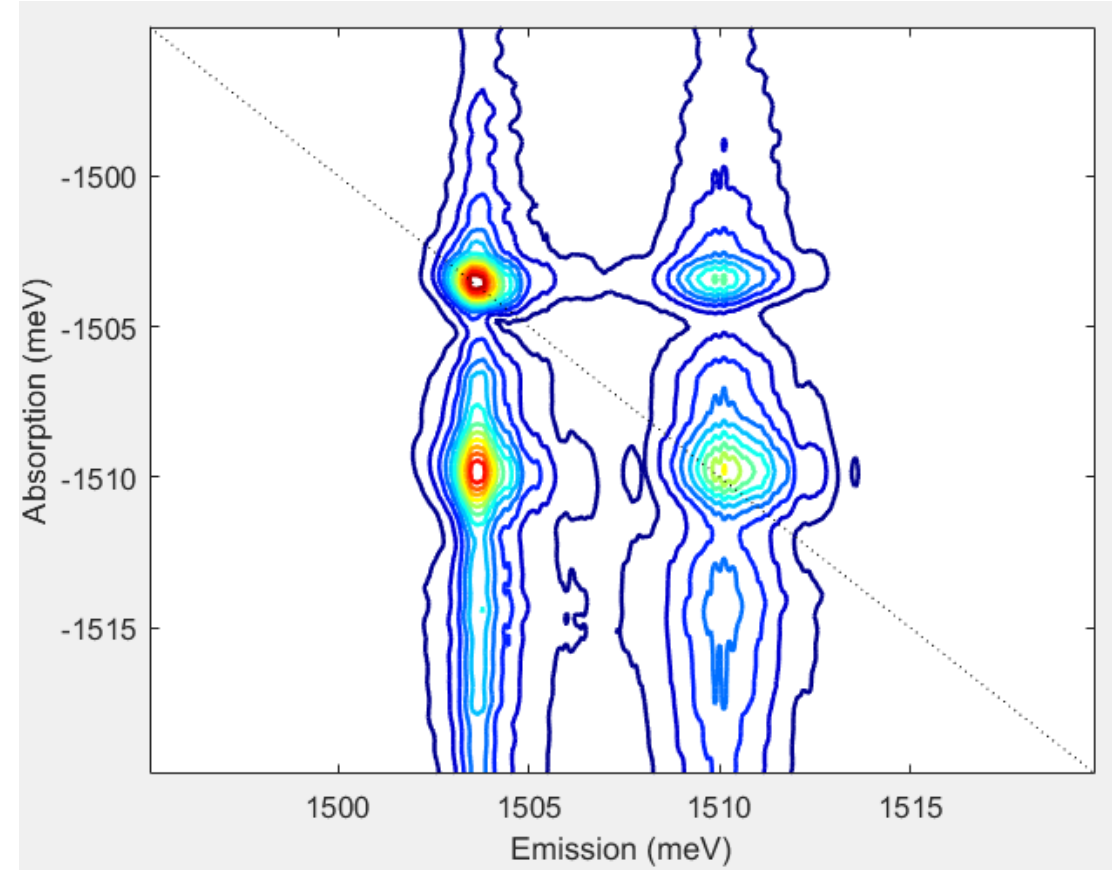

Figure 36 The 2D amplitude spectrum from a completed scan

Finally, the two dimensional spectrum is displayed. If the scan is still in progress, this includes the current number of "steps.". Figure 35 and Figure 36 show 2D amplitude spectra 
resulting from an unfinished and finished scan, respectively. Notice how the peaks are widened and take on a different shape compared to the end result. This data is the most sensitive to changes in the interferograms and when the contour lines stop changes as the scan processes this indicates without a doubt that the scan is finished.

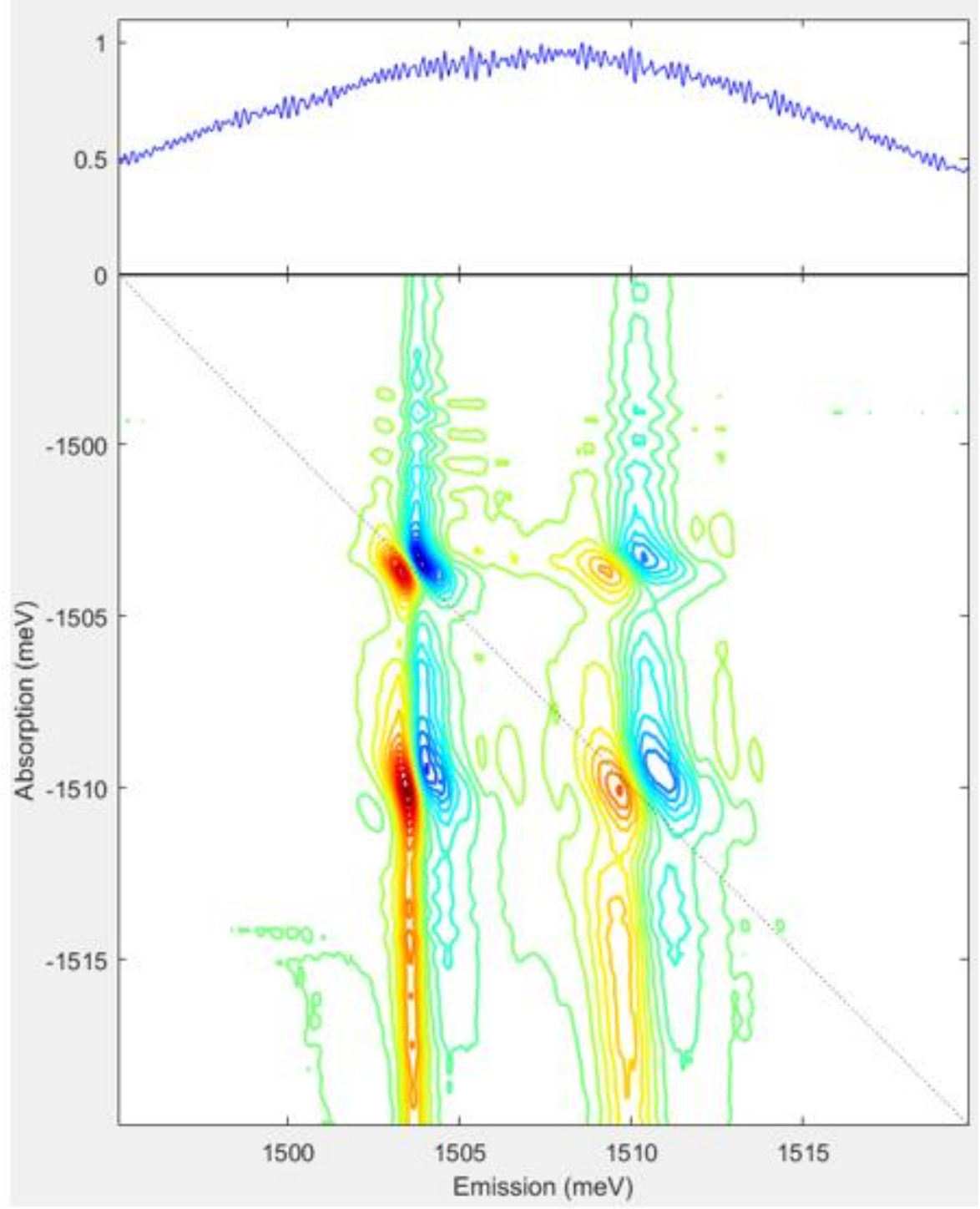

Figure 37 (upper panel) the reference pulse spectrum (lower panel) the real part of the $2 \mathrm{D}$ complex spectrum, after processing the phase

\subsection{Gallium Arsenide Excitons}

Gallium Arsenide is a direct bandgap III-IV semiconductor with a zinc-blend crystal structure, with a bandgap in the near infrared $\sim 1.423 \mathrm{eV}$ and a lattice constant of $5.658 \AA \AA$ at room 
temperature. It has been used to grow a wide range of heterostructures ranging from bulk samples to those with diminishing dimensionality such as quantum wells and quantum dots.

\subsubsection{Excitons}

Inside GaAs, and many other systems, when an electron transitions to an excited state it leaves behind a vacancy in the electronic density of states of the valence band. This vacancy can be treated as a positively charged particle and called a hole. The excited electron will exist for a lifetime before recombination with the hole, emitting a photon and returning back to the valence band. Before recombining, however, the electron and hole, with sufficient wavefunction overlap, can bind to form another quasiparticle called an exciton. It is the first excited state of the system. It is hydrogen-like because it consists of one positive and one negative charge that form a metastable alignment due to the mutual electrostatic potential with a minimum at a finite interparticle spacing. One can define Rydberg constant $R_{X}$ which is modified by the reduced masses of the electronic particles and the local dielectric constant and $n$ energy levels [130]

$$
\begin{gathered}
E_{X, n}=\frac{-R_{X}}{n^{2}}, n \geq 1 \\
R_{X}=\frac{\hbar^{2}}{2 m_{r} a_{X}^{2}}
\end{gathered}
$$

with Bohr radius

$$
a_{X}=\frac{\epsilon \hbar^{2}}{m_{r} e^{2}}
$$

In Bulk GaAs, $R_{X} \approx 4.1 \mathrm{meV}$ (the binding energy of the $\mathrm{n}=1$ state (ground) state), with $a_{X}=11.6 \mathrm{~nm}$, relative permittivity $\epsilon=12.9$, and reduced mass $\mu=\frac{m_{e} m_{h}}{m_{e}+m_{h}}=.059 m_{0}$. The free electron mass is $m_{0}$, and the effective masses of the electron and hole are $m_{e}=.0662 m_{0}$ and $m_{h}=.51 m_{0}[131,132]$. Figure 38 shows energy levels $(n=1,2, \ldots)$, the electron-hole continuum $E_{g}$ (band gap energy) at the gamma point, and parabolic dispersion away from $k=$ $0 \mathrm{~m}^{-1}$. The lowest is energy level is $E_{X}(k=0)$, giving the binding energy of the exciton and has $\mathrm{n}=1$, similar to the $1 \mathrm{~s}$ hydrogenic wavefunction. Additional bands are shown for $n=2$, 2 s-like 
wavefunction and $n=3$, 3s-like wavefunctions. $N=\infty$ also corresponds to the unbound e-h continuum.

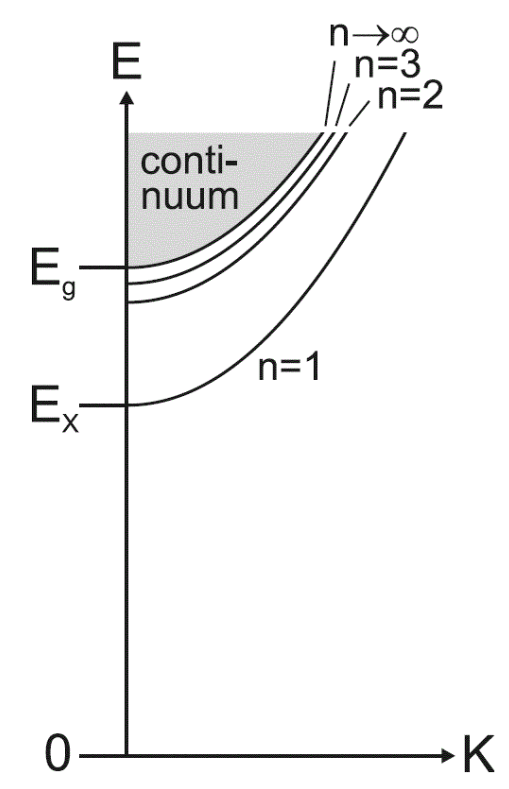

Figure 38 Rydberg energy levels of excitons, reproduced from reference [130].

Since these are bound exciton states, excitonic energies actually lie slightly below the conduction band, in the band gap, offset by an amount equal to their binding energy. Resonant excitation of excitons is possible, as well as relaxation from unbound electron and hole pairs . Thus it is possible to preferentially create excitons over their unbound counterparts with resonant excitation and a sufficiently narrow excitation bandwidth.

In the same way that excitons are atomic-like correlations of electron and hole pairs, excitons can also bind like neutral atoms to form molecular like wavefunctions. For example, a biexciton is a 4 particle correlation ( 2 holes and 2 electrons). Biexcitons have their own binding energy and ladder of excited states. Higher order correlations are indeed possible depending on the local electronic environment, leading to triexciton, excitonic droplets, excitonic Bose condensates and excitonic liquids $[24,88,105,133-137]$. Within this scheme there is a hierarchy of few- and many-body interactions and quantum statistics that can be explored. Generally, it continues until the binding energy is too weak to hold the molecule together against other forces such as interactions with other charged particles or phonons. 
From an application standpoint, excitons can be used as absorbers and emitters in a wide range of photonic devices for example since the exciton is almost a textbook dipole.

\subsubsection{Bulk GaAs Excitons}

GaAs has two degenerate $L=1$ ( $p$-type) valence bands at the valence band edge, where $L$ is the orbital angular momentum quantum number. In terms of the total angular momentum $J$ and its projections along the $z$-axis $J_{z}$, the bands belong to the $J=3 / 2$ states with $J_{z}=$ $\pm 3 / 2$ and $\pm 1 / 2$. These projections describe the heavy hole $(h)$ (with $J_{z}= \pm 3 / 2$ ) and light hole $(l)\left(U_{z}= \pm 1 / 2\right)$ bands, respectively, because the two states have different effective masses owing to the different momenta. A third valence band exists, called the split off band resulting from spin-orbit coupling, and has $J=1 / 2$ and $J_{z}= \pm 1 / 2$. However, it is far (340 meV) from the band edge and not excited in our measurements. Though its presence necessitates the use of $J$ and $J_{Z}$ as quantum numbers because the split-off term in the Hamiltonian doesn't commute with $L$ and $S$. The conduction band has $L=0$ (s-type) and $J=1 / 2$ and $J_{z}= \pm 1 / 2$. A diagram of these bands is shown in the Figure 39 [138], that figure is in the exciton picture.

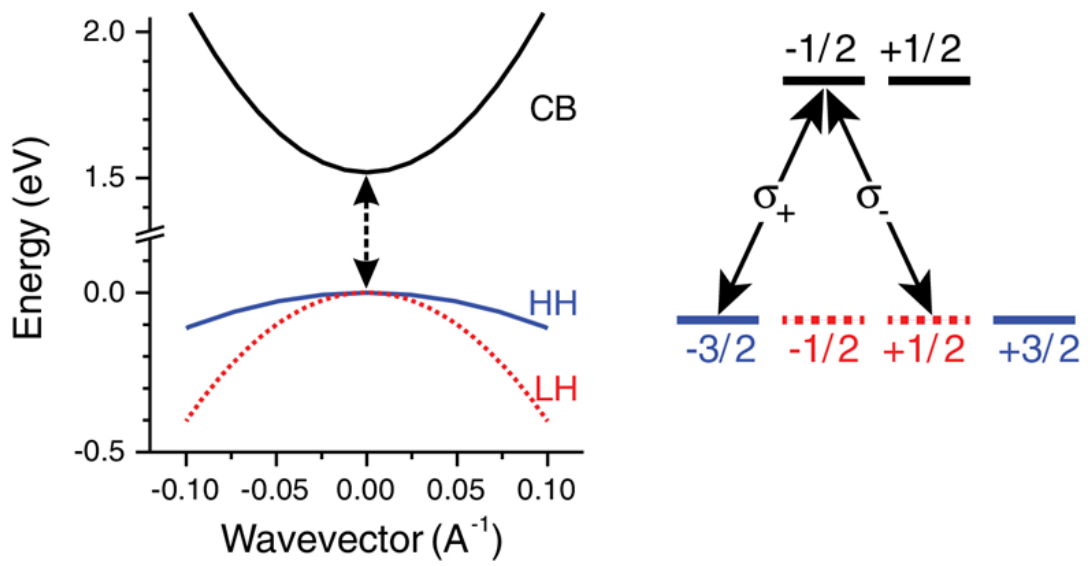

Figure 39 (a) The band structure of bulk GaAs (b) the electronic energy levels along with the $z$ component of their total angular momentum $\left(J_{Z}\right)$. The conductions, heavy-hole valence, and light-hole valence bands are labeled $C B, H H$, and $L H$, respectively. Reproduced from reference [138].

Polarization dependence of the dipole selection rules are shown as well on the right. To transition from a $-3 / 2$ state in the valence band to a $-1 / 2$ state in the conduction band, right hand 
circular polarization is needed to impart the correct $\hbar$ amount of momentum and vice versa for states decreasing in momentum. Thus to study the effect of polarization, it is ideal to have polarization control of the separate beams used in multi-beam measurements.

GaAs has been shown to support a hierarchy of exciton-correlated states, from the single exciton up to three bound excitons forming triexcitons [24,121]. The MDCS setup in the present work is capable of resolving multi-excitonic effects, which are sensitive to polarization owing to the spin coupling of the excitons forming the bound states. Selection rules for excitons and biexcitons are shown in Figure 40(a) $[49,139]$ where the basis has been transformed from the single-particle electron and hole picture to the two- particle exciton picture. The ground state $|g\rangle$ represents no excitation.

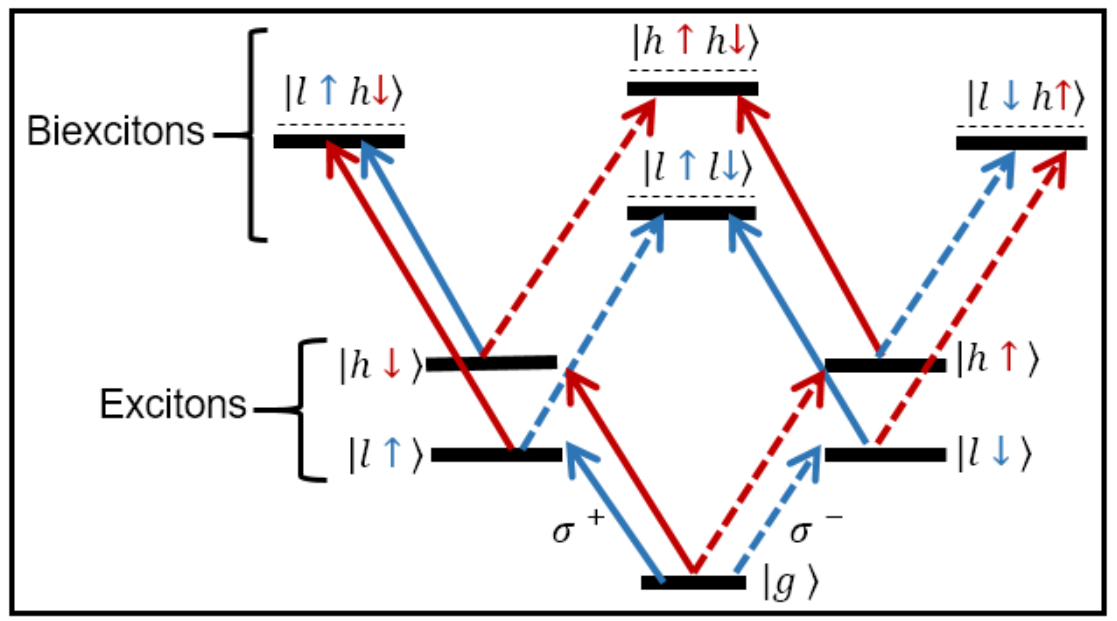

Figure 40 Energy level diagram and polarization selection rules for an excitonic system with degeneracy broken heavy- and light- hole states. Red and blue arrows indicate heavy- and lighthole transitions, respectively. Solid and dashed arrows indicate transitions from absorbing right $(+\hbar)$ and left $(-\hbar)$ polarization, respectively. Reproduced from reference [139].

As biexcitons have a binding energy ranging from a fraction of an meV to at most a couple of meV, depending on the type of sample structure, the nonlinear MDCS experiments are able to clearly resolve biexcitonic contributions because of detection in a background free direction owing to the sensitivity of the nonlinear response to phase matching to detect weak signals as well as the unique spectral positions bound higher excitonic molecules take in two-dimensional 
spectra, which (at a very basic level) allow differences between absorption and emission to be visualized.

\subsubsection{Strain}

Under normal circumstances the two valence states of bulk GaAs are degenerate, however the degeneracy can be broken. In lower dimensionality structures, the energies are mass-dependent as in a quantum well, and degeneracy is broken. These are frequently grown with breaking that degeneracy as the expressed purpose. An easier method is to utilize strain.

The exciton peak positions may be understood taking into account the hydrostatic and uniaxial stress components, the latter of which lifts the degeneracy in the valence bands. The uniaxial strain breaks the degeneracy of the valence bands and the hydrostatic portion shifts the whole bandgap. These effects are shown in Figure 41 [130]. The hydrostatic part lowers the bandgap if it is compressive and widens it if tensile. Whether the uniaxial out of plane strain is compressive or tensile will determine the ordering of the valence bands. Compressive uniaxial strain moves the light hole band to lower energy (widening the transition) while tensile does the same but to the heavy hole band. The valence bands experience warping and anticrossings where they would overlap away from $k=0$, see Figure 41(c). 
(a)

(b)

(c)

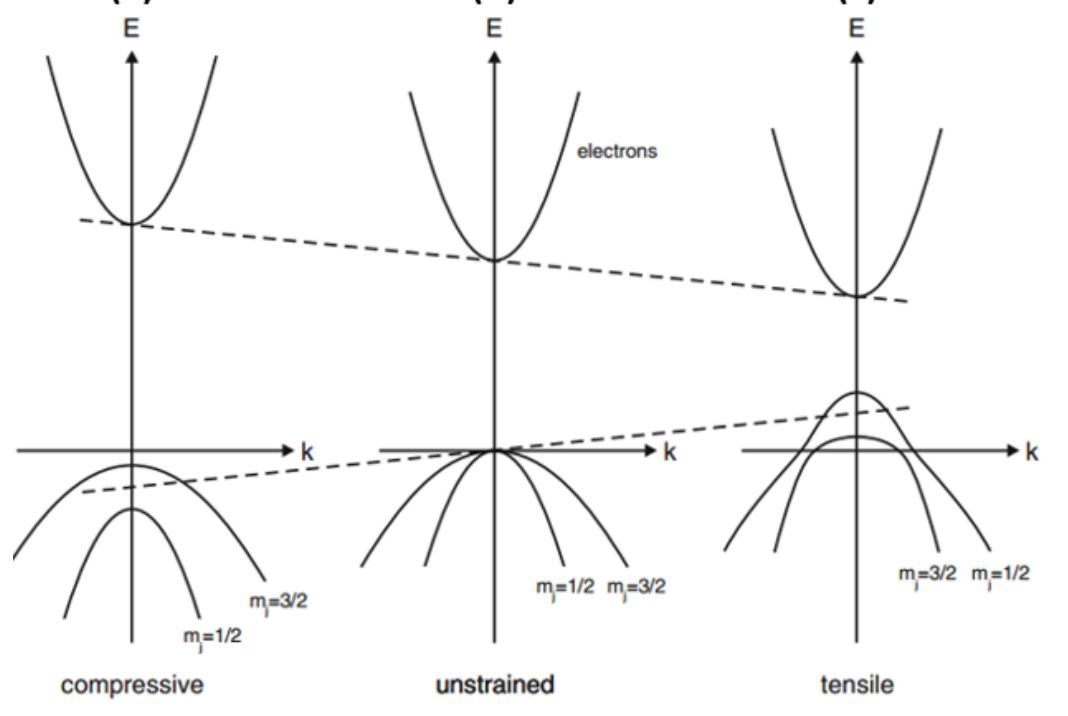

Figure 41 The effects of (a) compressive (b) zero and (b) tensile uniaxial strain on the system also under hydrostatic strain. $\mathrm{K}$ is the crystal momentum. Reproduced from [130].

To understand the implications of strain on quantum applications the entire response, including coherent properties, must be understood. Coherent nonlinear optical techniques, such MDCS, provide a more comprehensive picture of the excitonic response compared to linear optical techniques. MDCS may be exploited to gain further insight into the influence of strain on the coherent optical response of semiconductors. For instance, the ability to independently measure the levels of homogeneous and inhomogeneous broadening [29,60-64] may yield insight into varying local electronic environments tied to partial strain relaxation in thin film systems. MDCS has also provided a wealth of information about many-body interactions in quantum well systems through the ability to isolate a range of quantum pathways from the excitons, their coupling to one another, and biexcitons [19,21,25,26,28,49,50,65-68]. The study of such effects in a bulk semiconductor system, in which the splitting between the exciton resonances is caused exclusively by strain, would provide insight into the influence of strain on $\mathrm{MBI}$ and the coherent response of the excitons [69].

In this work, MDCS is used to study bulk excitons in two GaAs layers with different thicknesses, such that the two samples exhibit different amounts of strain, resulting from being attached to sapphire disks and cooled. Comparison of rephasing MDCS spectra with collinear 
and cross-linear polarization configurations allows for the observation of the line shapes, center positions, homogeneous and inhomogeneous linewidths, and the coupling of $l, h$ excitons and biexcitons. In addition to increasing the separation between the $h$ and $l$ excitons, these experiments show that increasing the amount of strain in the bulk semiconductor modifies the relative strengths of the off-diagonal exciton features, altering the spectrum from a coherent response typically seen in atomic vapors to one seen in quantum wells. A larger degree of inhomogeneous broadening is also observed in the sample with larger strain, attributed to variations in the local electronic environment that are likely tied to strain gradients along the growth direction of the heterostructure. Significant bound biexciton signatures are visible for cross-linear polarized excitation, due to the presence of few-body interactions. Overall, the results suggest that MDCS and the coherent response of GaAs excitons is highly sensitive to strain.

\subsection{Exciton-Polaritons}

\subsubsection{The Quantum Well}

The reference of excitons in this particular sample is $\ln .{ }_{04} \mathrm{Ga} .{ }_{96} \mathrm{As}$, which has a bulk lowtemperature bandgap of $\sim 1457 \mathrm{meV}$ and non-degenerate heavy and light hole states because the incorporation of indium into the crystal introduces strain which breaks the degeneracy, similar to that of the strained bulk GaAs discussed earlier [140-143]. It is grown thin enough, via molecular beam epitaxy, to exhibit quantum mechanical effects because it is spatially confined in one dimension on the order of the Bohr radius of the excitons $(8 \mathrm{~nm})$. This arrangement is the experimental realization of a textbook quantum well. Strictly speaking it is a finite quantum well. However, the GaAs barriers are sufficiently high enough and thick enough that infinite quantum well formalism is a good, and intuitive, approximation. Figure 42 (a) shows the infinite quantum well and (b) shows a simple diagram of the real well structure, where cited energies are bulk values for the band gaps. The steady-state solutions to the time independent Schrödinger wave equation $H \psi=E \psi$ subject to the infinite well barrier potentials are standing waves $\psi_{n}(z, t)$ with eigenenergies $E_{n}$ : 


$$
\begin{gathered}
\psi_{n}(z, t)=A \sin \left(k_{n} z\right) e^{-i \frac{E_{n}}{\hbar} t} \quad n=1,2, \ldots \\
E_{n}=\frac{\hbar^{2} k_{n}^{2}}{2 m}=\frac{\hbar^{2} \pi^{2} n^{2}}{2 m L^{2}}, \quad k_{n}=\frac{n \pi}{L}
\end{gathered}
$$

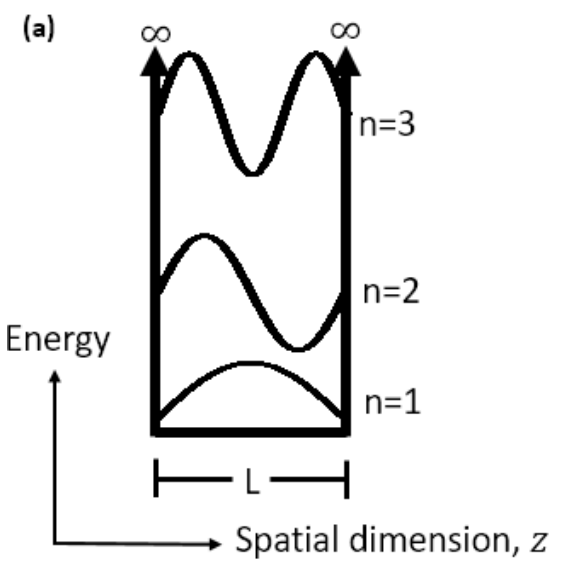

(b)

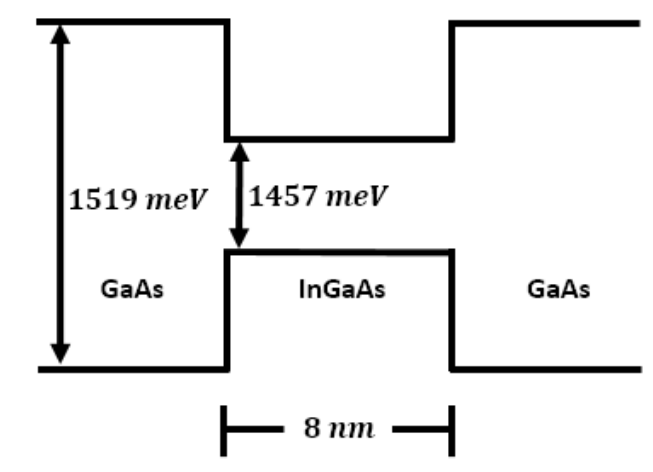

Figure 42 An (infinite) quantum well, of thickness $L$ with infinitely high barriers

Because the eigenenergies depend on mass, the degeneracy between the heavy and light hole valence bands (and thus their excitons) is broken and additionally because of the spatial confinement all energies move upward. Low temperature absorption data for this sample is shown in Figure 43 [143-146].

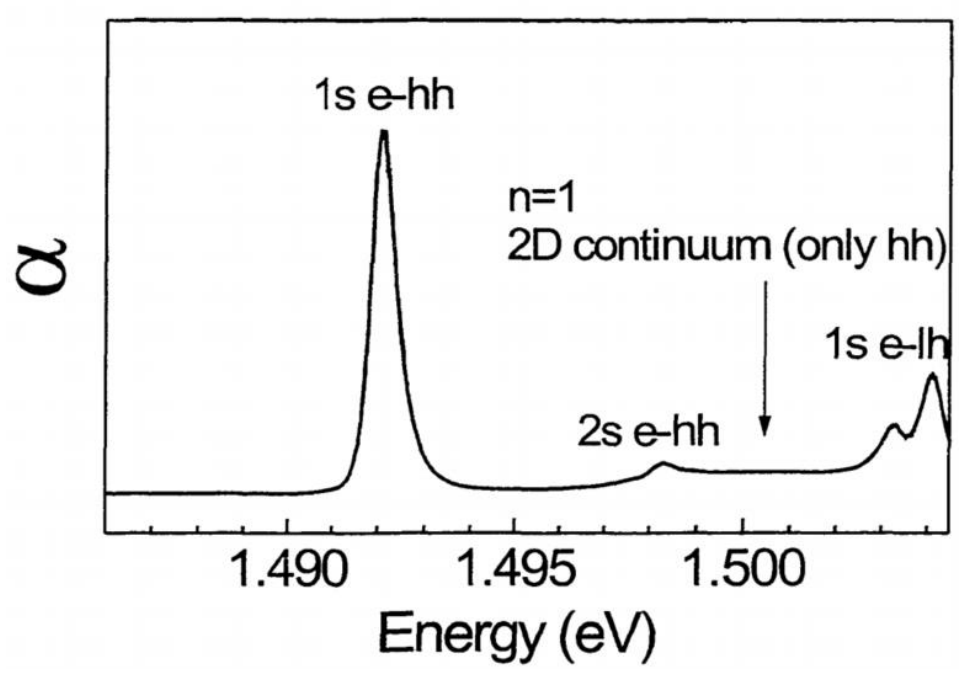

Figure 43 Absorption coefficient of $\ln .04 \mathrm{Ga} .96$ As quantum well at low temperature $(4 \mathrm{~K})$. The $1 \mathrm{~s}$ electron(e)-heavy hole (hh) and the $2 \mathrm{~s}$ e-hh transitions are seen, as well as $1 \mathrm{~s}$ e-light hole (Ih). Only the 1s hh exciton resides in the well. Reproduced from [143]. Note that this is the sample used in this work, labeled NMC73. 
The heavy hole, 1 s exciton in this $8 \mathrm{~nm}$ InGaAs well sits at $1492.3 \mathrm{meV}[43,143,147]$ and is the only exciton which resides in the well. A strong light hole peak is visible at $~ 1504.2 \mathrm{meV}$. Two peaks on the low and high energy side (cut off) of the 1s light hole peak are due to dark excitons and possibly a $2 \mathrm{~s}$ light hole exciton [148-152]. As a result of being in the well, the exciton experiences increased binding energy and oscillator strength, the latter of which is evidenced by the relatively strong peak strength. The former is evidenced by a redshift of the emission energy compared to bulk (not shown). In addition to these effects, spatial confinement simply confines the excitons to a narrow region inside the cavity, which is essential for maximizing the cavityexciton coupling.

\subsubsection{The Quantum Microcavity (QMC)}

Under normal circumstances (without photonic enhancement) a quantum well's excitons couple weakly to the vacuum field-they absorb and emit photons to the continuum but not couple strongly enough to photons in order to form a bound pair, i.e. an exciton-polariton, because the two do not coexist long enough and the probability of interacting is not high enough. A resonant, planar Fabry- Perot type cavity overcomes these problems by concentrating the electric field intensity (the cavity mode) and increasing the time the photons coexist spatially with the excitons as the photons are confined in the cavity for several round-trips [153]. Purcell predicted, and observed in NMR measurements, that coupling between the normal mode of a cavity and a dipole could modify spontaneous emission rates, known as "Purcell enhancement" [154]. Later, an isolated atom interacting with a resonant cavity without an applied external field was shown to also exhibit splitting and modification of spontaneous emission rates. The lack of an applied field lead to the term "vacuum-field Rabi splitting" to describe the coupling $[155,156]$ which continues to be used even in the presence of applied fields, sometimes modified to just "Rabi splitting". It was since proposed that the effect is equally applicable to solid-state semiconductors [157] and NMC between quantum well excitons and a microcavity's modes were observed by Weisbuch et al. in 1992 [86]. The cavity itself is called a microcavity because the heterostructure's size is generally on the order of microns and it becomes a quantum microcavity with the incorporation of a quantum well or wells. 
The quantum well is sandwiched between two GaAs spacers in turn sandwiched between two high-reflecting mirrors, as in Figure 44, and the whole heterostructure is grown via molecular beam epitaxy on a GaAs substrate [95]. The quantum well is drawn to reflect realistic imperfections during growth that result in width variations. Therefore, excitons in different spatial regions will have slightly different eigenenergies - a significant reference of inhomogeneous dephasing in QM wells ultimately passed onto the polaritons. The mirrors are distributed Bragg reflectors (DBRs), each consisting of alternating layers of high and low index of refraction material $\frac{\lambda}{n 4}$ thick, where $\lambda$ is the resident cavity mode wavelength and $n$ is the index of refraction of the respective layer. Each interface between the bilayers generates a Fresnel reflection (and the larger the contrast in index the larger the reflection coefficient) which interferes constructively with reflections from subsequent layers generating high reflectivities and quality factors on the order of $\sim 10^{3}[153,158]$. These bilayers are GaAs and AIAs, with indices of refraction 3.66 [159] and 2.99 [160] at $830 \mathrm{~nm}$, respectively. There are 14.5 and 12 bilayers on the bottom and top respectively such that the emission emerges predominantly in one direction. A fractional layer on the bottom is because it terminates on a GaAs substrate (which takes the place of the GaAs half of the bilayer). 


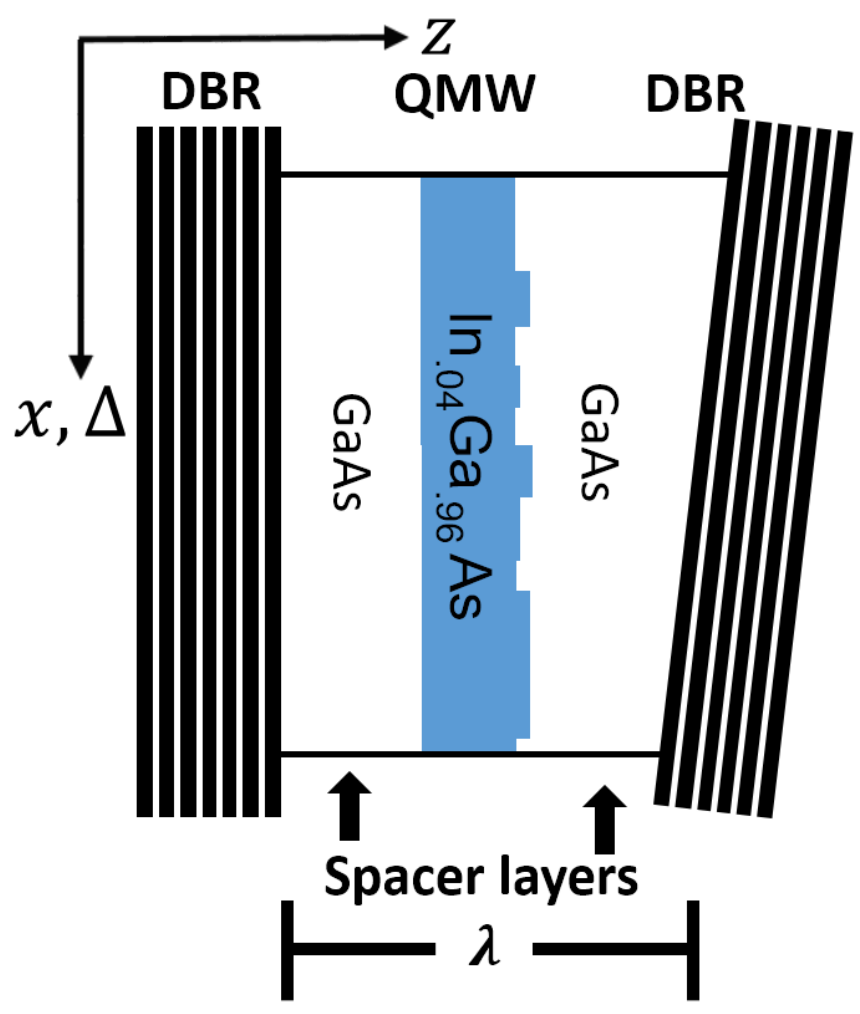

Figure 44 Quantum microcavity structure consisting of an InGaAs quantum well (QMW) placed at the antinode of a wedged microcavity resonator consisting of two DBRs and GaAs spacers (not to scale).

The cavity is also wedged to allow the cavity mode to be tuned (or detuned, rather) with respect to the nearly constant exciton energy of the quantum well by merely moving the sample in the laser focus along the $\mathrm{x}$ axis. The detuning is $\Delta=E_{C}-E_{X}$, where $E_{C}$ is the resonant cavity mode energy and $E_{X}$ the exciton energy. It could also be expressed in wavelength or frequency, or, sometimes more practically in a laboratory setting, as sample position since different points on the sample have different cavity energies due to the wedge shape.

At room temperature, one can observe the behavior of the microcavity alone as thermal fluctuations prevent exciton formation in the well. Figure 45 shows the room temperature reflectivity of the present sample as a function of wavelength. The DBRs generate nearly $100 \%$ reflectivity in what is called the stop band, for $100 \mathrm{~nm}$ from $\sim 870-970 \mathrm{~nm}$ at this particular point on the sample. The cavity mode generates a dip in the middle of the stop band near $905 \mathrm{~nm}$ where light of that wavelength is allowed to enter (and eventually leave) the cavity. This dip is 
the ideal spectral position for the well, or any absorber whose transitions one wishes to couple to the cavity.

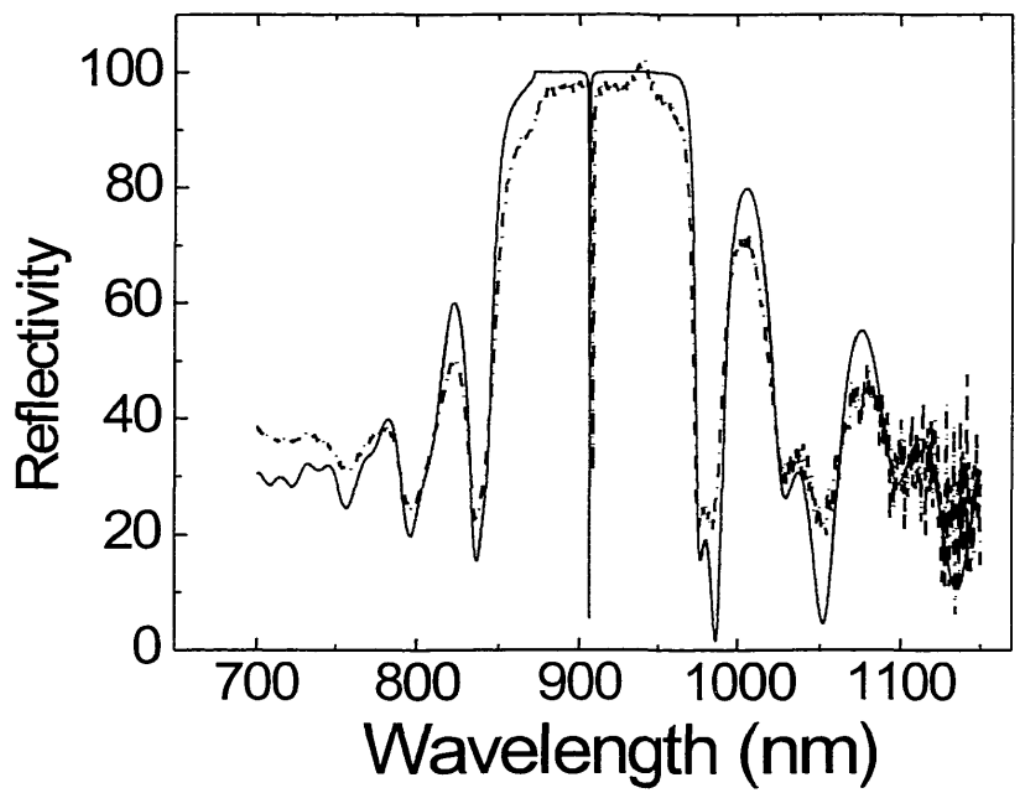

Figure 45 Reflectivity of microcavity NMC73 at room temperature showing the stop band from $\sim 870$ to $\sim 970 \mathrm{~nm}$ and dip at $\sim 905$ caused by the cavity mode. The solid (dashed) line is calculated (measured) reflectivity. Reproduced from [143].

Looking at the reflectivity at low temperature ( $7 \mathrm{~K})$ and varying the detuning close the quantum well energy, one observes two dips where there was previously one as in Figure 46 moving spectrally. These are the upper and lower polariton branches, with upper referring to the higher energy (shorter wavelength) peak. From top to bottom, as the detuning is moved positively, the branches move to shorter wavelengths while the splitting decreases to a minimum value and then begin to separate again. This is the well-known anti-crossing behavior of a strongly coupled cavity-oscillator system. 


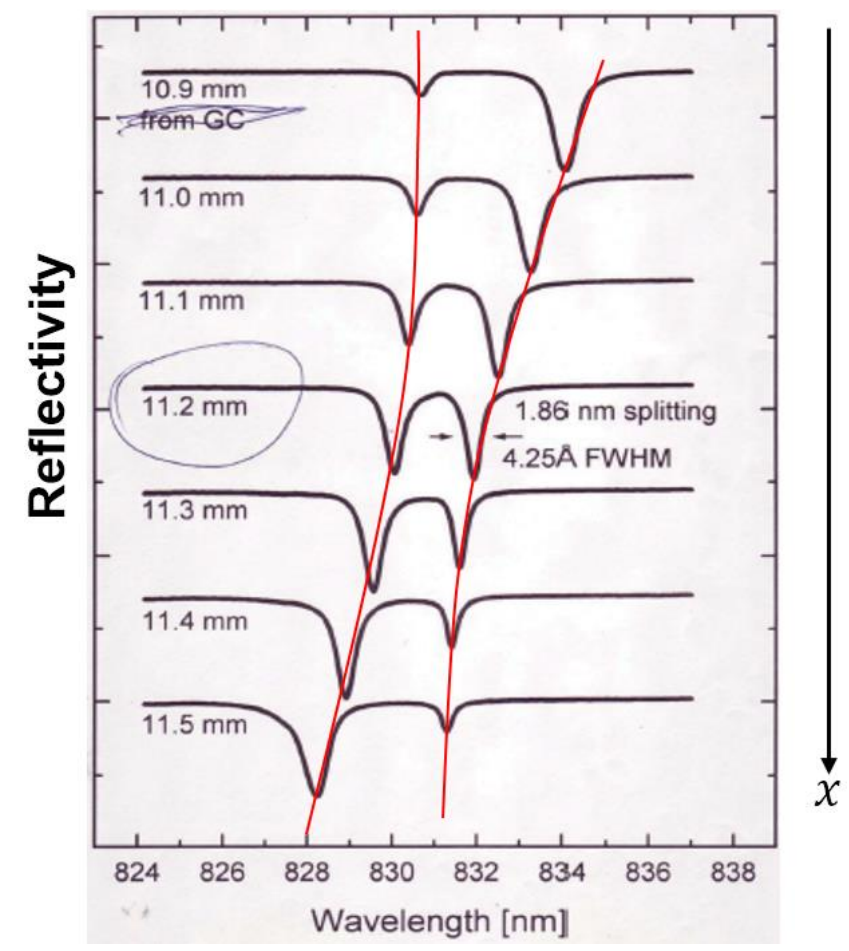

Figure 46 Quantum microcavity reflectivity detuning dependence of the reflectivity showing two dips moving spectrally that are the upper (UP) and lower (LP) polariton branches. Modified from reference [161].

The degree of coherent coupling has been defined as a threshold in atomic systems. Saturation atomic $N_{0}$ and photons numbers $n_{0}$ necessary to strongly couple are $[153,162]$ :

$$
\begin{gathered}
N_{0} \propto \frac{\gamma}{\tau g^{2}} \propto \frac{V}{Q} \\
\text { and } \\
n_{0} \propto \frac{\Gamma \gamma}{g^{2}} \propto V
\end{gathered}
$$

Where $\gamma$ and $\Gamma$ are the atomic dephasing and population rates respectively, $\tau$ the cavity lifetime, $V$ the mode volume, and $Q$ the cavity's quality factor. The oscillator-field coupling strength $g$ is related to mode volume by $[153,163]$ :

$$
g=\sqrt{\frac{\mu^{2} \omega_{c a v}}{2 \hbar \epsilon_{o} V}}
$$


Where $\mu$ is the oscillator's dipole transition moment. A measure of the degree of the final coupling is the minimum separation between the polariton branches as the detuning is varied, called the Rabi splitting and related to the coupling strength as $E_{R}=2 \hbar g[162,164]$. With a quantum well it is modified, in its simplest form, as $E_{R}=2 h \sqrt{\frac{f_{O S C .} \omega_{\text {cav }} N_{Q W}}{L_{\text {cav }}}}$, where $f_{\text {OSC. }}$ is the exciton oscillator strength, $N_{Q W}$ the number of quantum wells, and $L_{c a v}$ the cavity length [164168]. In all cases, stronger coupling generates stronger Rabi splitting which is brought about by small cavities, high quality factors, and strong dipole moments.

A final and important property of polaritons is that they have properties of both the constituent cavity photons and the quantum well excitons that depends on the detuning. The Hopfield coefficients [169-171], $\left|X_{k}\right|^{2}$ and $\left|C_{k}\right|^{2}$, communicate what percentage excitonic or photonic, respectively, the polariton is. The coefficients for the lower polariton branch (and similarly for the upper branch) are:

$$
\begin{gathered}
X_{k, L P}=\frac{1}{\sqrt{1+\left(\frac{E_{R}}{E_{L P(k)}-E_{C}}\right)^{2}}} \\
C_{k, L P}=-\frac{1}{\sqrt{1+\left(\frac{E_{L P(k)}-E_{C}}{E_{R}}\right)^{2}}}
\end{gathered}
$$

and normalized such that $\left|X_{k}\right|^{2}+\left|C_{k}\right|^{2}=1$, where $E_{L P}$ is the lower polariton branch energy and with wavevector $k$. The general behavior is simple: for example, starting at negative detuning $\left(E_{C}<E_{X}\right)$ and moving positive values, the lower branch goes from cavity like to excitonic and vice versa for the upper branch. At zero detuning both branches are equal parts excitonic and photonic, which makes intuitive sense the cavity is resonant with the excitons allowing the strongest coupling. This behavior will be demonstrated further in Chapter 5. 


\section{Chapter 4 Bulk Gallium Arsenide Excitons-Results}

In this chapter we will explore bulk GaAs samples under nominal strain. Specifically, 2DCS measurements are performed on two cooled bulk layers of different thicknesses at varying amounts (two) of strain, carrier density, polarization, and temperature.

Samples of intrinsic GaAs are grown by molecular-beam epitaxy to have thicknesses of 300 $\mathrm{nm}$ (grown by Margaret Dobrowolska, Xinyu Liu, and Jacek K. Furdyna at the University of Notre Dame) and $800 \mathrm{~nm}$ (grown by Richard P. Mirin at NIST Boulder) along with etch stop layers to protect the sample from chemicals used for removing material from the sample later. The samples are glued to sapphire disks using mounting wax and the GaAs substrates are removed with chemical etching for optical transmission measurements. All measurements were performed below $10 \mathrm{~K}$ in a cold finger cryostat, where thermal contact between the sapphire substrate and the sample mount allows the heat to be sunk from the sample.

Optical absorption, SR-FWM, and MDCS measurements were performed with resonant excitation, which is in the range of $1506-1512 \mathrm{meV}$ for the present study. The pulses are attenuated using neutral, optically dense, absorptive filters to an average power of $0.2 \mathrm{~mW}$ per beam. The phase-matching condition selects the rephasing spectrum with the pulse ordering $A^{*}$, $B, C$, where the star denotes the phase-conjugated pulse.

All excitation and emission pulses are set with either collinear (XXXX) or cross-linear (XYYX) polarization configurations. The first three terms (XYY for example) indicate the exciting beams' $(A, B$, and $C$ ) polarization states and the fourth term indicates the emission state, chosen via analyzers at the spectrometer input. [66] The laser pulses' tuning are spectrally centered over the midpoint between the $h$ and $l$ excitons observed in the linear spectra in order to provide roughly equal spectral flux for each state. The sample is moved into the focus of the three excitation beams by optimizing the strength of the FWM signal at zero delay time. Irises are centered and closed around the phase matched direction to minimize noise due to scatter of the pump pulses into the spectrometer. 


\subsection{SR-FWM}

Figure 47 shows an example of the emission spectrum of the third-order response of excitons (spectrally resolved FWM) in the $800 \mathrm{~nm}$ thick bulk GaAs sample, taken under collinear $(\mathrm{XXXX})$ polarization and low excitation power and temperature, .2 $\mathrm{mW}$ per beam and $10 \mathrm{~K}$, respectively [139].

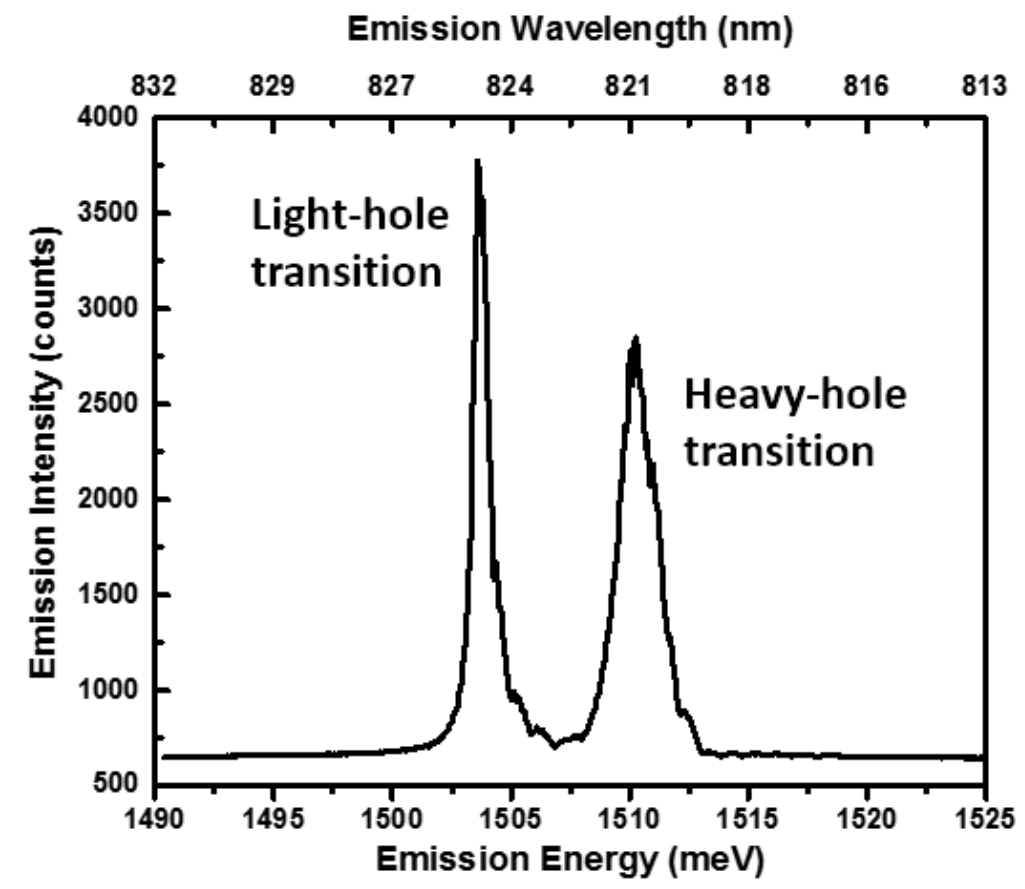

Figure 47 Spectrally resolved FWM of excitons in $800 \mathrm{~nm}$ thick bulk GaAs

There are two peaks, rather than one which would be occur when looking at an unstrained bulk GaAs sample because of the degeneracy of the heavy- and light- hole valence bands, see Figure 41(b) and (c). However, strain has broken that degeneracy in this system. Though from this spectrum alone, it is not possible to determine much information about what type of strain the system is experiencing mostly because it is not possible to identify which peak is the heavy or light hole (the labels have been placed after the fact). To do that, we will perform a simple calculation of the lattice constrictions as the sample is cooled as well as look at absorption spectra of two samples of different thicknesses. 


\subsection{Absorption Measurements and Strain Splitting}

Figure 48(a) shows the linear absorption of the 300-nm (blue) and 800-nm (red) GaAs layers. The spectra show sharp resonances due to the strain-split excitons and electron-hole continuum at higher energies. The thinner and thicker sample exhibit a splitting between the exciton resonances of $\sim 6$ and $\sim 3$ meV, respectively. 


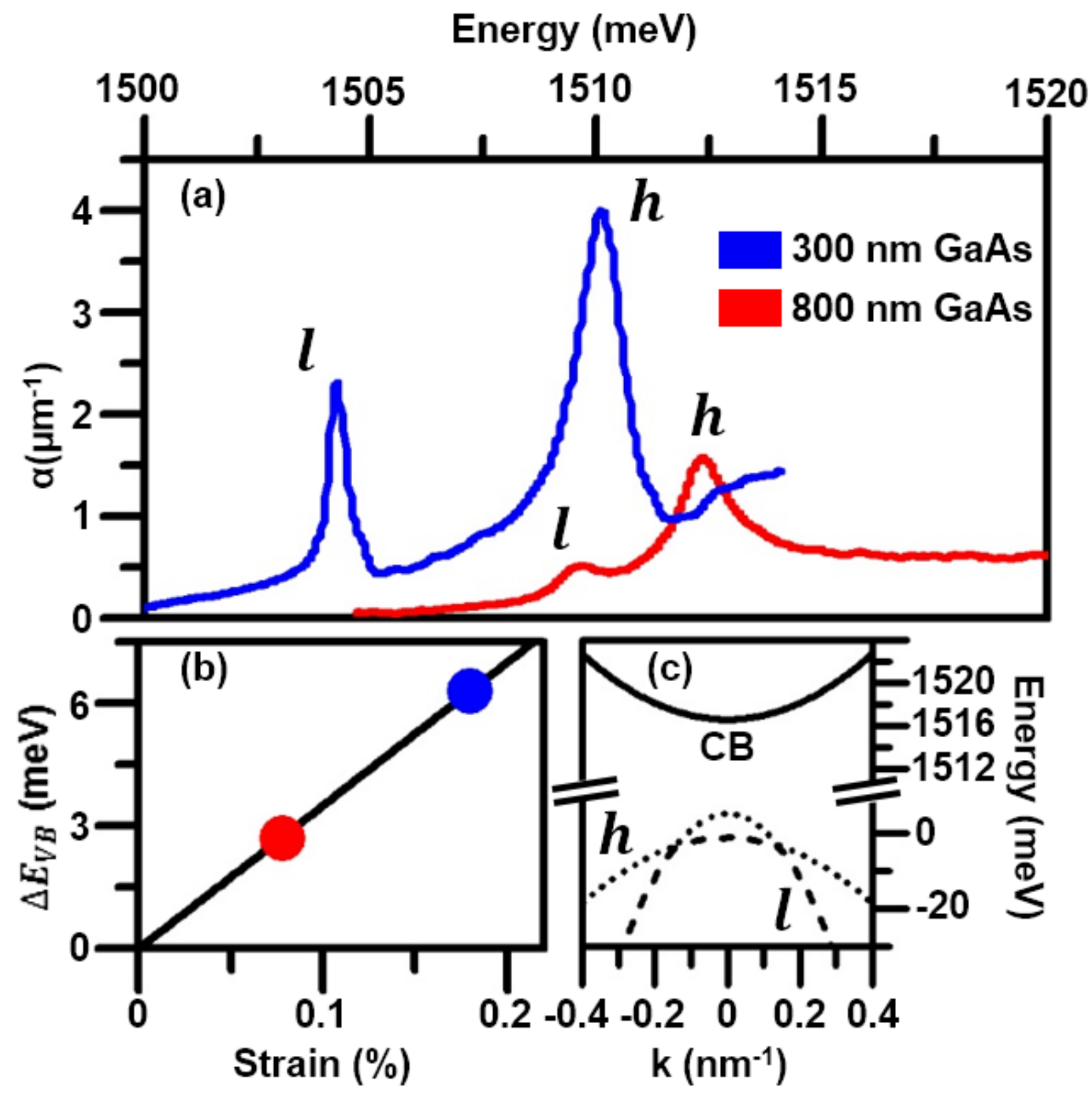

Figure 48 (a) Linear optical absorption spectra for a 300-nm (high strain) and 800-nm (low strain) GaAs layers revealing strain-split heavy- $(h)$ and light-hole $(l)$ excitons. (b) Effective strain $(\Delta \mathrm{e})$ versus the exciton splitting $(\Delta \mathrm{E})$ for both samples. (c) Single-particle dispersions of the conduction, $h$, and $l$ valence bands. Modified from reference [139]. 
Cooling leads to uniform contraction of the sapphire. The different coefficients of thermal expansion for GaAs and sapphire results in two-dimensional in-plane tensile strain of the thin GaAs layers. [172] At room temperature, bare bulk GaAs has a thermal expansion coefficient of $5.8 \times 10^{-6} / \mathrm{K}$, using this number the lattice constant will contract $.167 \%$ from $5.658 \AA$ at room temperature to 5.649 Angstroms. The gap also shifts as the sample is cooled according to the empirical Varshini equation, $E_{g}=1519 \mathrm{meV}+.5405 \frac{T^{2}}{T+204}$ to about $1519.253 \mathrm{meV}$ at $10 \mathrm{~K}$, though this shift is not due to strain, rather just the normal contractions seen when phonons, which on average increase the lattice constant, are frozen out by cooling. [173] The sapphire substrate contracts less than the GaAs, having a coefficient of thermal expansion on the order of $7-8 \times 10^{-6} / \mathrm{K}$ at high temperature and decreasing to $\sim 10^{-12} / \mathrm{K}$ at cryogenic temperatures. The room temperature lattice constant of bare sapphire is $4.758 \AA$ and $4.756 \AA$ at low temperature $[174,175]$. However, the large variance in the reported values for the coefficient of expansion for sapphire over the temperature range it was cooled made theoretical calculation of its exact contracted lattice constant unreliable and the low temperature $(4.756 \AA)$ literature value is used. Though it is clear that the sapphire contracts less ( .04 \%). When the two materials are rigidly attached and cooled, this difference ( $.127 \%)$ leads to biaxial tensile strain in the plane of the sample which relaxes over the growth direction (the substrate is of course strained too). This estimate of the lattice mismatch as a function of temperature does not take into account how constriction would be affected by rigidly, mechanically attaching one material to another, as well as how different material thicknesses would affect the system. As we will demonstrate, these effects are significant. But the important result of this calculation is that we know the GaAs is under tensile in-plane strain, cause by the outward force of the substrate.

This in-plane strain necessarily causes slight compressive strain along the growth direction, in an attempt to maintain the GaAs unit cell volume. Strain is able to relax along the growth direction away from the point of attachment to the sapphire. The two samples possess different effective strain values, as well as differing variations in the local electronic environment due to differing amounts of strain relaxation along the growth direction. 
It is known that strain, among other variables, alters the band structure. Hydrostatic and biaxial tensile strain lift the degeneracy and puts the LH band energetically higher than the $\mathrm{HH}$ band. Perturbative strain linearly shifts the bands by an amount given by $E_{\text {shift }}=\Xi(\Delta a / a)$, where $\Xi$ is the deformation potential, $a$ is the lattice constant, and $\Delta a$ is the change in lattice constant due to stress. [176] Uniform biaxial in-plane strain lifts the degeneracy of the $h$ and $l$ valence bands, given by $\Delta E=2|b| \Delta e,[82,83]$ where $b$ is the shear deformation potential, and $\Delta e=e_{x x}-e_{z z}$ is the effective strain difference between the in-plane and out-of-plane directions. Using the literature value $b=-1750 \mathrm{meV}$, [177] effective strain differences of $0.18 \pm$ $0.01 \%$ and $0.08 \pm 0.01 \%$ are determined for the thinner and thicker GaAs layers respectively; see Figure 48 (b). In the figure, the error bars are smaller than the size of the data markers.

Conduction $\mathrm{cb}\left(E_{c b}\right), h\left(E_{h}\right)$ and $l\left(E_{L l}\right)$ bands are determined to have quadratic dispersions, [178] which are modified by linear shift terms at the $\Gamma$-point and band mixing away from $k=0$. Final equations within an effective 1-D model are simplified by assuming $e_{x x}=e_{y y}$, giving:

$$
\begin{gathered}
E_{c b}=\frac{\hbar^{2}}{2 m_{c}} k^{2}+E_{g}+a_{c}\left(2 e_{x x}+e_{z z}\right) \\
E_{l}=-\frac{\hbar^{2}}{2 m_{e}} \gamma_{1} k^{2}+a_{v}\left(2 e_{x x}+e_{z z}\right)+\left|\frac{\hbar^{2}}{2 m_{L}} \gamma_{2} k^{2}-b\left(e_{x x}-e_{z z}\right)\right|
\end{gathered}
$$

And

$$
E_{h}=-\frac{\hbar^{2}}{2 m_{e}} \gamma_{1} k^{2}+a_{v}\left(2 e_{x x}+e_{z z}\right)-\left|\frac{\hbar^{2}}{2 m_{H}} \gamma_{2} k^{2}-b\left(e_{x x}-e_{z z}\right)\right|
$$

Literature values for the effective masses of the $c b, l$, and $h$ bands are $m_{C}=0.066 m_{e}, m_{L}=$ $0.094 m_{e}$ and $m_{H}=0.34 m_{e}$ respectively, [179] where $m_{e}$ is the elementary electron mass. The unstrained low-temperature band gap is $E_{g}=1518.9 \mathrm{meV}$, [173] valence band deformation potential is $a_{v}=1600 \mathrm{meV}$, conduction band deformation potential is $a_{c}=-7000 \mathrm{meV}$ and the Luttinger parameters are $\gamma_{1}=6.98$, and $\gamma_{2}=2.06$. [72] The band gap is determined from the onset of the electron- $l$ continuum in Figure 48 (a) to be $\sim 1512.8 \mathrm{meV}$. These values are used in conjunction with the observed exciton splitting, fitting parameters $e_{x x}$ and $e_{z z}$ to yield strains of $0.07 \%$ and $-0.11 \%$ in the $x y$ plane and $z$ directions respectively for the high-strain sample. The 
resulting band edges are $1516.6 \mathrm{meV}, 4.7 \mathrm{meV}$, and $-1.5 \mathrm{meV}$ for the $c b, l$ and $h$ bands respectively. Equations (4.1)-(4.3) are plotted Figure 48(c) taking into account the literature and observed parameters. Away from the band $\Gamma$ point, anti-crossings appear where the $h$ and $l$ bands are in close proximity. Although high wave vector regions of the dispersions are out of the accessible range of wave vectors used in the experiment. Within the light cone, near the point, $E_{l}>E_{h}$, such that the lower energy spectral feature is the $l$ exciton. Similar analysis can be performed for the low-strain sample.

\subsection{Feynman Diagrams of Strain-Split Excitons}

The third-order response of the excitonic system can be described by double-sided Feynman diagrams that traverse the available levels for the quantum system $[49,116,180]$. Figure 49(a) shows the level diagram arising from a Hilbert space transform of the single-particle electronic states to the two-particle exciton states in bulk GaAs, including the spin degeneracy and strain splitting. Optical transitions are shown in the circular basis, where $\sigma^{-}$and $\sigma^{+}$ correspond to left (red) and right (blue) circular polarization. Additionally, four biexcitons denoted $|h h\rangle,|l l\rangle,|h l\rangle$ and $|l h\rangle$. The latter two are mixed biexcitons consisting of a bound $h$ and $l$ pair. In the diagrams, the bound biexcitons are drawn as solid lines below the dashed lines to illustrate the sub-meV bulk biexciton binding energy. [181] The dotted line is the energy required to create the two bare excitons constituting the bound biexciton and the resulting bound state sits below that by the binding energy, which can be calculated as the difference between the dotted and solid lines. Note that the spin quantum number has been omitted for ease of discussion of linearly polarized excitation. Since linear polarization can be expressed as a superposition of circular polarization, the pathways traversed by excitation in the linear basis is a sum of Feynman diagrams in the circular basis. 

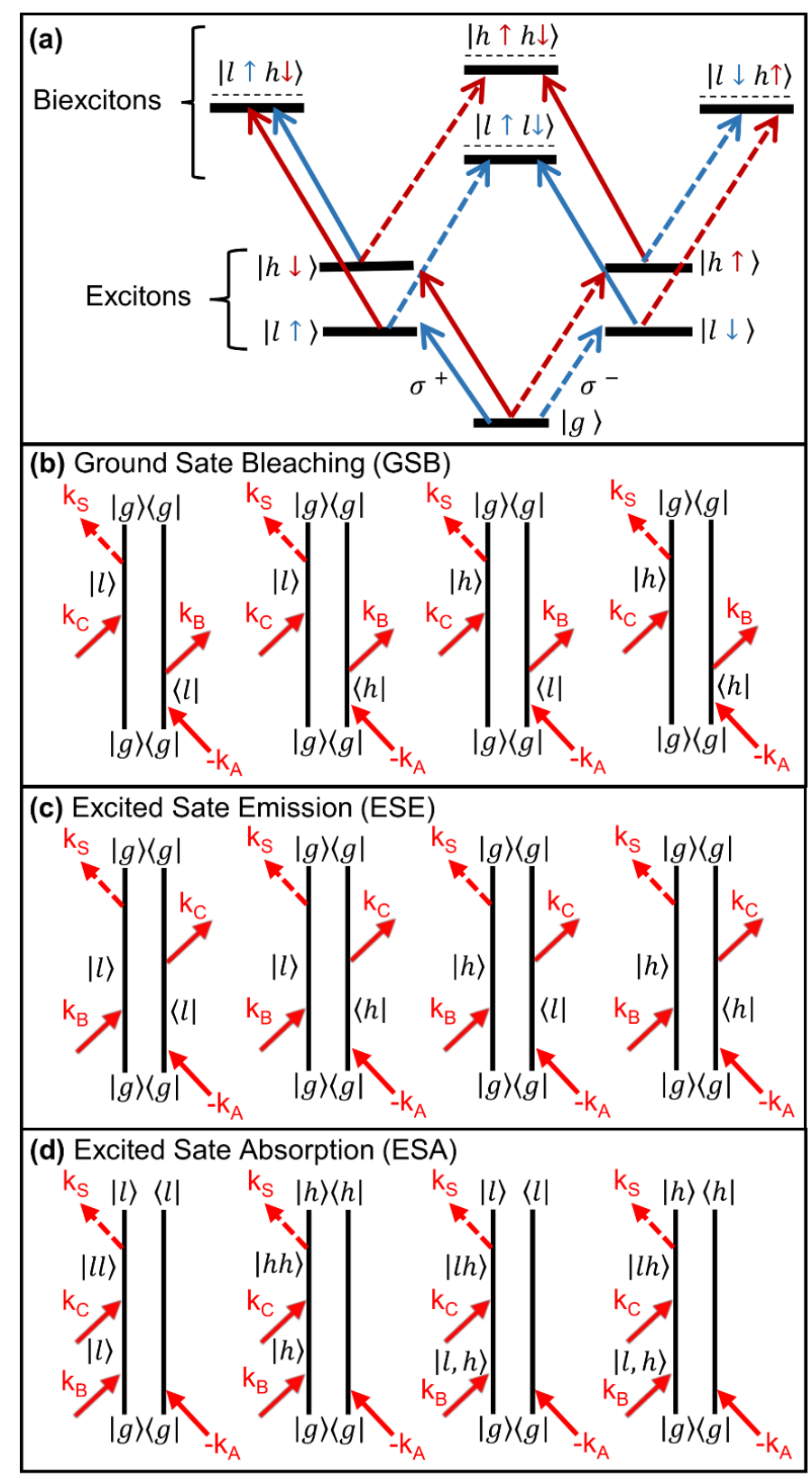

Figure 49 (a) Exciton level diagram for strain-split bulk excitons and biexcitons in GaAs. Doublesided Feynman diagrams illustrate the quantum pathways of (b) ground state bleaching, (c) excited state emission and (d) and excited state absorption. Reproduced from reference [139]. 
Quantum pathways traversing the level diagram obey time-ordering and the phasematching condition corresponding to rephasing spectra [49]. Signals must occur from excitation by all three pulses; hence, the language of polarization and population can be employed as a result of successive interactions with odd and even numbers of laser pulses, respectively. Figure 49(b) shows ground-state bleaching (GSB) pathways, where the second pulse creates a population in the ground state. Figure 49(c) shows excited-state emission (ESE) pathways, where the second pulse drives a population/polarization in the first excited state (excitonic) manifold. In MDFTS, spectra both the GSB and ESE pathways result in diagonal intra-action features for both exciton species and two off-diagonal interaction features that are a result of coupling between the two exciton species. Figure 49(d) shows excited-state absorption (ESA) pathways, where the signal arises from a final populating of the excited state and includes a polarization between the excitonic and second-excited (biexcitonic) manifolds. In MDFTS spectra, ESA contributions can be used to identify the bound biexcitons, because the biexciton binding energy results in a shift in the emission energy relative to the absorption energy. This results in an offset along the emission axis to lower energy and moves the peak away from the diagonal. To date MDFTS has revealed biexciton features adjacent to the heavy-hole exciton in GaAs quantum wells, $[65,66]$ interfacial quantum dot distributions, [182] and upper branch exciton-polaritons in semiconductor microcavities (discussed in Chapters 3 \& 5). [43]

\subsection{Two-Dimensional Correlation Spectra}

Figure 50 shows the MDFTS rephasing spectra for the low- $(0.08 \%)$ and high-strain $(0.18 \%)$ samples: (a) amplitude and (e) real parts of the XXXX polarized spectra are shown for the lowstrain sample; (b) and (f) show the same for XYYX polarization. Similarly, high-strain spectra are show in panels (c), (d), (g) and (h). All spectra are normalized to their strongest feature. First, it is worth discussing the spectral features that are common to both samples. Each spectrum is dominated by four star-like features as described above, namely two diagonal $(l$ and $h)$ and two off-diagonal $(l-h)$ and $(h-l)$ coherences. In addition, XXXX polarized spectra for both samples show two vertical streaks (denoted $C_{l}$, and $C_{h}$ ), which are coherences resulting from $\mathrm{MBI}$ between the electron-hole continuum and the $l$ and $h$ resonances. $[69,183]$ Exciting with XYYX polarization is known to suppress $\mathrm{MBI}$ due to excitation induced dephasing, $[69,184,185]$ such 
that in MDFTS spectra the signals are reduced by a factor of approximately five, the $C_{l}$ and $C_{h}$ continuum features are strongly reduced, the off-diagonal features become similar or weaker than the diagonal features, $[65,66]$ and biexciton features become more prominent. $[66,182]$ The latter effect is more pronounced for the high strain sample. For both samples, dispersive line shapes are observed in the real part of the XXXX polarized spectra as a result of the MBI. $[21,65]$ For XYYX polarization, with suppressed $\mathrm{MBI}$, the spectral features become peaked, more absorbative, about their center position. For the case of the diagonal features in the real MDCS spectra, this means they are centered on the diagonal with positive sign, while off-diagonal features are centered at positions corresponding to absorption into one exciton species and emission from the other with negative sign. 


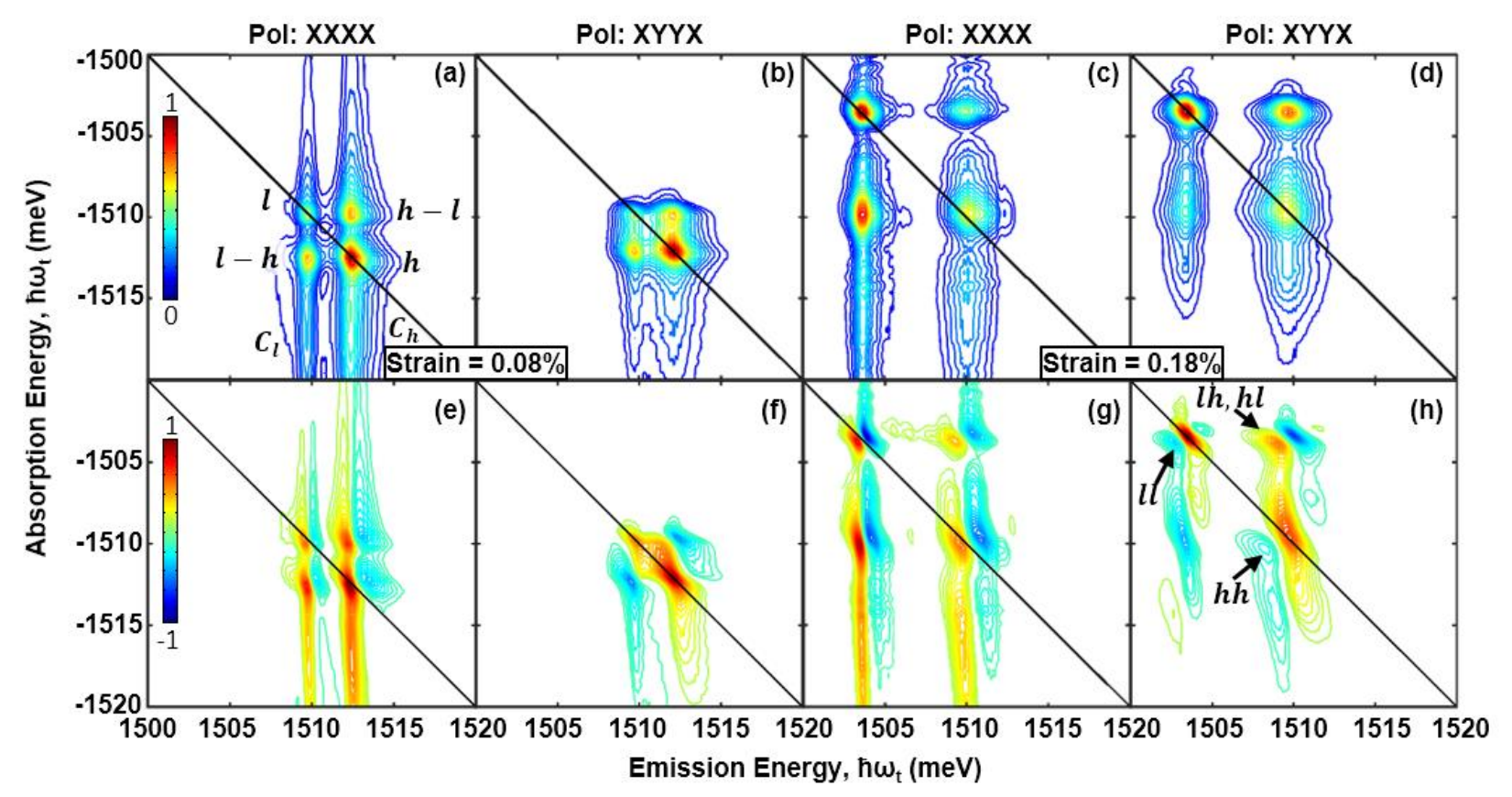

Figure 50 Rephasing 2DCS spectra for XXXX and XYYX polarization of the low-strain (0.08\%) and high-strain (0.18\%) GaAs samples. Panels (a)-(d) show the normalized amplitude and panels (e)(h) show the corresponding real part of the spectra. Diagonal (intra-action) coherences $l$ and $h$ are the light- and heavy- hole excitons respectively, off-diagonal (interaction) coherences $l-h$ and $h-l$ reveal coupling between exciton species. Features $C_{l}$ and $C_{h}$ are electron-hole continuum coherences with their respective excitons. Features $l l, h h$, and $l h, h l$ correspond to light-, heavy-, and mixed- biexciton coherences in the high-strain sample. Modified from reference [139]. 
Increasing the strain from $0.08 \%$ to $0.18 \%$ has several effects on the coherent properties observed in the 2DCS rephasing spectra. As seen in the linear spectra, the splitting between the two exciton resonances increases with strain. The spectral coordinates $\left\{E_{a b s}, E_{e m i}\right\}$ of the four main features $l, h, l-h$, and $h-l$ are listed in Table 1 for both low- and high-strain samples. The increased splitting has the effect of making the exciton species more energetically distinct as is evidenced by an alteration in the spectral weights of the four main features of Figure $50(a)$ and (c). It can be seen that in the low-strain sample the $h$ has larger oscillator strength than $l$, and that $l-h$ and $h-l$ are equal in magnitude, with strengths that are approximately the average of $l$ and $h$. This result is reminiscent of the coherent response of three-level atomic vapors, [47] indicating a symmetric coupling between the two exciton species. [49] On the other hand, in the high-strain sample the lower energy exciton $l$ is stronger than $h$ and the relative strengths of the off-diagonal features become asymmetric, with $l-h$ increasing by approximately the same amount that $h-l$ decreases. This asymmetry is a signature of the excitation-induced dephasing and excitation-induced shift. [21,183] This is reminiscent of the coherent response in lower dimensional GaAs systems and not incoherent energy relaxation, since $T=100 \mathrm{fs}$. The comparison of the two results suggest a greater impact of $\mathrm{MBI}$ in the high-strain sample. More study is required to elucidate the role of $\mathrm{MBI}$ in the two samples. [69]

Another prominent effect of the increased strain is the presence of new features in $X Y Y X$ rephasing spectra, which tend to be more sensitive to bound biexciton contributions that arise from ESA pathways; see Figure 49(d). The real-part of XYYX spectrum in Figure 50 (h) exhibits a strong negative $h h$ feature. Spectral coordinates $\left\{E_{a b s,} E_{e m i}\right\}$ are listed in Table 2, revealing the shift in the emission energy that corresponds to the biexciton binding energy. This contribution arises from the ESA pathway shown second to left in Figure 49 and has been observed in other excitonic systems as discussed above. In addition, new features are observed. The feature at $l l$ is associated with a light-hole biexciton and the left most double-sided Feynman diagram in Figure 49(d). The feature at $l h, h l$ is associated with a mixed biexciton and the right most double-sided Feynman diagram of the same series. Energetically, the mixed biexciton pathway involves pulse $A^{*}$ creating a polarization between $|g\rangle$ and $|h\rangle$, pulse $B$ creating a polarization in either $|h\rangle$ or $|l\rangle$, pulse $C$ creating a polarization with $|l h\rangle$ or $|h l\rangle$ and emission releasing a photon with energy $E_{h}-$ 
$\Delta E_{l h, h l}$ to return the system to a population in $|h\rangle$. From the lateral shifts of the three biexcitonic features determined from the spectral coordinates listed in Table 2, it is estimated that the binding energies are $\sim 1 \mathrm{meV}$ and $\sim 2 \mathrm{meV}$ for the $\mathrm{LH}$ and $\mathrm{HH}$ biexcitons, which compare well to literature values. [179] Averaging the two pure biexciton binding energies gives a value close to observed mixed biexciton binding energy, $\Delta E_{l h, h l}=1.2 \pm 0.1 \mathrm{meV}$. The presence of the mixed biexciton enhances the $h-l$ feature in the normalized amplitude spectra for this polarization (see Figure 50(d)), as compared to the EID and EIS enhancement of the $l-h$ feature for XXXX polarization (see Figure 50(c)). To the best of the authors' knowledge, mixed biexcitons have been theoretically explored in one-quantum rephasing [49] and two-quantum 2DCS and experimentally observed in two-quantum spectra, $[50,51]$ but have not been directly observed in one-quantum rephasing spectra. Biexciton contributions are not observed in the low-strain sample, likely because of the relatively small $h / l$ splitting which is comparable to the biexciton binding energy. 
Table 2 Spectral coordinates for the exciton and biexciton features in units of meV and with error of 0.1 meV. Exciton and biexciton features are extracted from XXXX and XYYX spectra respectively. The biexcitons were not discernable in the low strain sample and those entires are accordingly blank. Modified from [139].

\begin{tabular}{|c|c|c|c|c|}
\hline \multirow{2}{*}{$\begin{array}{l}\text { Spectral } \\
\text { Feature }\end{array}$} & \multicolumn{2}{|c|}{$\frac{\text { Low Strain }}{(0.08 \%)}$} & \multicolumn{2}{|c|}{$\frac{\text { High Strain }}{(0.18 \%)}$} \\
\hline & $\mathrm{E}_{\mathrm{abs}}$ & $\mathrm{E}_{\mathrm{emi}}$ & $\mathrm{E}_{\mathrm{abs}}$ & $\mathrm{E}_{\mathrm{emi}}$ \\
\hline$l$ & -1509.7 & 1509.7 & -1503.5 & 1503.5 \\
\hline$h$ & -1512.4 & 1512.4 & -1510 & 1510 \\
\hline$l-h$ & -1512.4 & 1509.7 & 1510 & 1503.5 \\
\hline$h-l$ & -1509.7 & 1512.4 & -1503.5 & 1510 \\
\hline$h h$ & - & - & -1510.2 & 1508.2 \\
\hline$l l$ & - & - & -1503.8 & 1502.8 \\
\hline$l h, h l$ & - & - & -1503.8 & 1509 \\
\hline
\end{tabular}


Star-like line shapes indicate that the exciton coherences have nominal inhomogeneous broadening, [62] as might be expected for bulk excitons where the excitonic Bohr radius is more than an order of magnitude smaller than the GaAs layer thickness. This contrasts with 2DCS spectra for excitons in low-dimensional semiconductors, where local variations in quantum confinement within the excitation spot lead to significant inhomogeneous broadening and spectral features that are elongated along the diagonal direction. Nonetheless, the degree of inhomogeneous broadening is acquired using the analysis method of Siemens et al. [62] which simultaneously fits the diagonal and cross-diagonal slices of each spectral feature, relating the line shapes to the homogeneous $(\gamma)$ and inhomogeneous $(\sigma)$ linewidths.

Table 3 shows the extracted linewidths from the $l$ and $h$ diagonal features for the four amplitude spectra shown in Figure 50(a) to (d). The error associated with each value from averaging is approximately $10 \%$ or $0.07 \mathrm{meV}$. Nonetheless, comparisons can be made for the two samples for the same polarization. All spectra show $\gamma \geq \sigma$ for both excitons, within the experimental uncertainty, indicating that the features are indeed all primarily homogeneously broadened. Moreover, the homogeneous linewidth of $l$ is smaller than that for $h$ when excited by XXXX polarization, where excitonic linewidths are less complicated. This result is consistent with visual inspection of both linear and 2DCS measurements. For both polarizations, the homogeneous linewidths of $l$ and $h$ are more comparable in the low-strain sample than the highstrain sample, consistent with the change in coupling discussed above. Namely, closer spaced energy levels lead to comparable line shapes, as well as comparable amplitudes of the offdiagonal elements. Similar changes in the distinguishability of 2DCS features has been observed in wedged semiconductor microcavities, [43] where the energy separation between lower and upper branches of the exciton-polaritons can be tuned to vary the interactions strength. For XYYX, biexcitons are expected to be similar strength to excitons, which complicates direct comparison of linewidths extracted from the two polarizations. This may also increase $\gamma$ for the low-strain sample. 


\section{Table 2}

Table 3 Homogeneous $(\gamma)$ and inhomogeneous $(\sigma)$ linewidths, with units of meV and an error of 10\%. Modified from reference [139].

\begin{tabular}{lccccc}
\hline \multicolumn{2}{r}{$\underline{\text { Strained }}$} & & \multicolumn{2}{c}{ XXXX } & \multicolumn{2}{c}{ XYYX } \\
$\underline{\text { excitons }}$ & $\gamma$ & $\sigma$ & $\gamma$ & $\sigma$ \\
\hline $0.08 \%$ & $l$ & 0.44 & 0.06 & 0.74 & 0.21 \\
& $h$ & 0.46 & 0.27 & 0.59 & 0.33 \\
$0.18 \%$ & $l$ & 0.29 & 0.34 & 0.29 & 0.27 \\
& $h$ & 0.70 & 0.41 & 0.87 & 0.31
\end{tabular}


Turning attention to the inhomogeneous broadening, it can be seen that in all cases $\sigma$ is larger for $h$ than for $l$, as has been observed in quantum wells [29]. The inhomogeneous broadening is also larger in the high-strain sample, which indicates a larger degree of variation in the microscopic electronic environment within the excitation volume for that sample. Since no variation is expected in the plane of the sample, the inhomogeneous broadening is attributed to strain relaxation in the growth direction $(z)$. Strain relaxation typically occurs on length scales larger than the exciton Bohr radius, [186] such that at any one point in the excitation volume the exciton's energy levels are related to the local potential. The lower the biaxial strain the smaller the splitting, the higher the strain the larger the splitting. Hence, a distribution of strain values experienced by the excitons locally in the sample is proportional to the average of the exhibited inhomogeneous broadening contribution to the linewidth in the diagonal direction of a 2DCS feature. In general, if a number of spectral features are observed this is given as $\sum_{N} W_{G, N} / N$, where $N$ is the peak index and $W_{G, N}$ is the Gaussian full-width half maximum (FWHM) of the peak, associated with inhomogeneous contribution to the diagonal Voigt profile [62] for the $N^{\text {th }}$ spectral feature. For moderate amounts of inhomogeneous broadening, the diagonal FWHM of the $N^{\text {th }}$ feature is described as $W_{N}=0.5346 W_{L, N}+\sqrt{0.2166 W_{L, N}^{2}+W_{G, N}^{2}}$, where $W_{L, N}=2 \gamma_{N}$ and $W_{G}=2 \sqrt{2 \ln 2 \sigma_{N}}$. [187] For $\mathrm{X}_{\mathrm{L}}$ and $\mathrm{X}_{\mathrm{H}}$ features, the average FWHM is $\left(W_{G, L}+W_{G, H}\right) / 2$, which yields $0.39 \pm 0.1 \mathrm{meV}$ and $0.88 \pm 0.1 \mathrm{meV}$ for the low- and high-strain samples respectively, based on ? values for XXXX polarization. Employing the same conversion used for the average splitting of the exciton line centers, the distribution in effective strain through the depth of the sample is $\{\Delta e\}_{z}=\left(W_{G, L}+W_{G, H}\right) /(4|b|)$ This yields $0.011 \%$ and $0.025 \%$ for the low- and highstrain samples respectively. Finally, if it is assumed that the strain relaxes uniformly over the thickness of each sample, then the effective strain relaxation length for the two samples is 139 $\% / \mathrm{cm}$ for the $800 \mathrm{~nm}$ thick layer and $841 \% / \mathrm{cm}$ for the $300 \mathrm{~nm}$ thick layer. These numbers reflect the situation that the thinner sample has larger strain, larger splitting between the excitons, a steeper strain relaxation gradient and larger contribution to inhomogeneous linewidth. 


\subsection{Power and Temperature Dependences in the High-Strain Sample}

Looking at just the highly strained sample, measurements were made as a function of power and temperature.

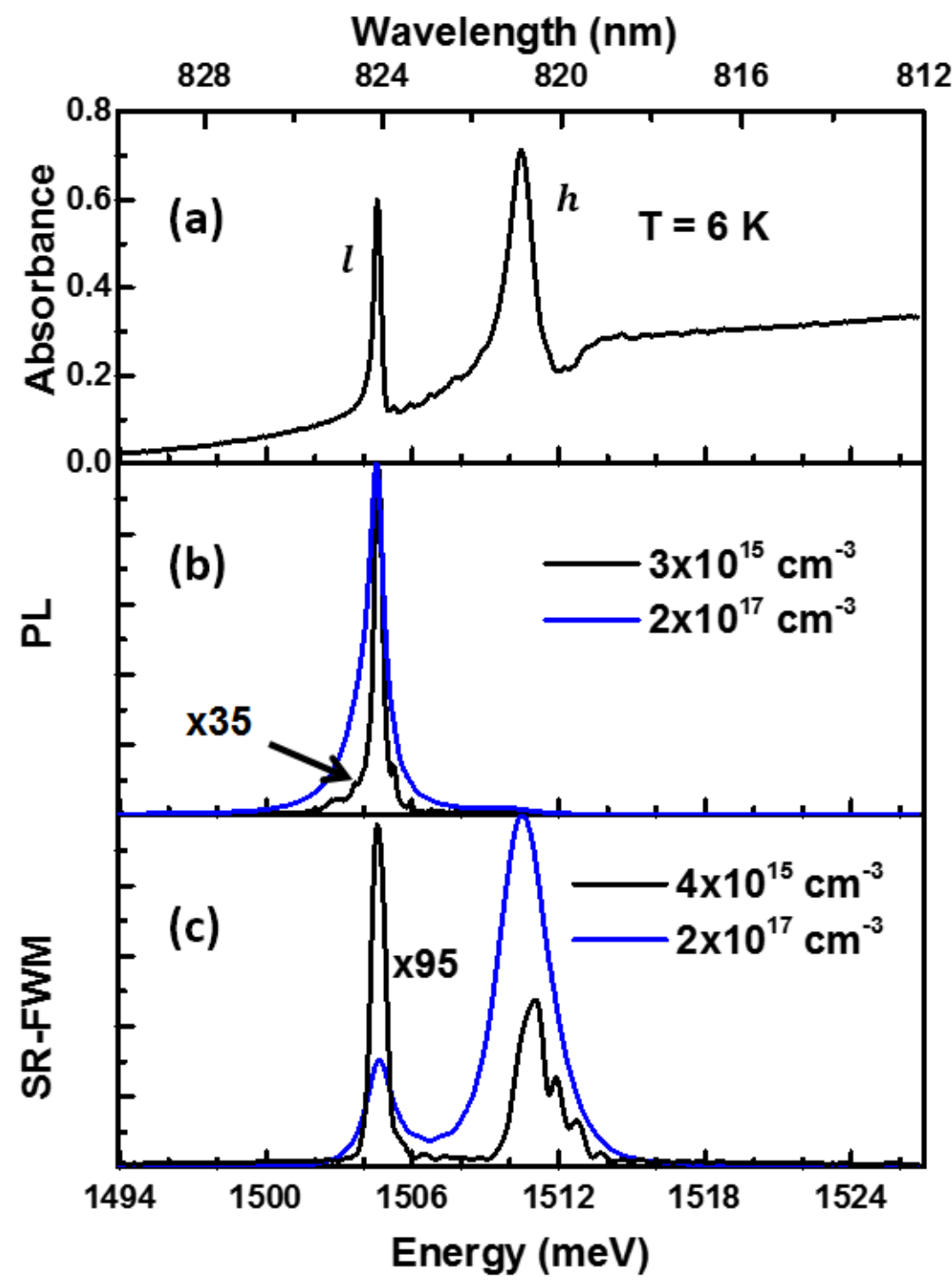

Figure 51 (a) Low-temperature absorbance and (b) Photoluminescence and (c) Four-wave mixing at low (black) and high (blue) excitation densities.

Figure 51 shows (a) absorbance, (b) photoluminescence (PL), and (c) SR-FWM data at 6K and the emission measurements were taken under low $\left(\sim 10^{15}\right)$ and high $\left(10^{17}\right)$ excitation densities. The PL and SR-FWM are normalized to the peak intensity and multipliers are indicated 
to communicate the relative amplitudes. Before discussing the power and temperature dependent properties, this is an opportunity to illustrate the different information contained in linear and nonlinear spectroscopy. The PL shows dominate emission from the lower energy state, the $l$, and very little radiation from the higher energy $h$ state. Whereas clear peaks are visible from both of the excitonic species in the nonlinear emission. Absorbance data in (a) also shows strong absorption at both of these energies, as well as the onset of continuum absorption at the band edge. Because of the time scales involved and only one interaction with the exciting field generates the $\mathrm{PL}$, the system has time to relax (which occurs on the order of nanoseconds) to mostly the lowest energy transition before radiatively recombining. For this reason, absorbance and FWM data is useful for elucidating the state structure.

Looking at the SR-FWM in Figure 51 (c), the positions of the $l$ and $h$ excitons are 1504.6 meV and $1510.98 \mathrm{mV}$, respectively. The relative intensity of the $l$ peak is about twice that of the $h$, and have widths of .52 and $1.61 \mathrm{meV}$, respectively. Because the temperature is $4 \mathrm{~K}$ lower than the data shown in Figure 47-Figure 50, there is a slight blue shift of all states and the band edge by $<1 \mathrm{meV}$. At high power, the relative strength of $l$ and $h$ swap, due to phase space filling of the lower energy LH states. A small excitation induced shift moves the $l$ and $h$ peaks to 1504.68 and $1510.54 \mathrm{meV}$, respectively. The peaks also widen to $1.01 \mathrm{meV}$ and $2.30 \mathrm{meV}$. The observed narrow linewidths are consistent with long dephasing times associated with little inhomogeneity, as substantiated by the MDCS measurements which are necessary to conclusively disentangle the convolved linewidths in one-dimensional data. 


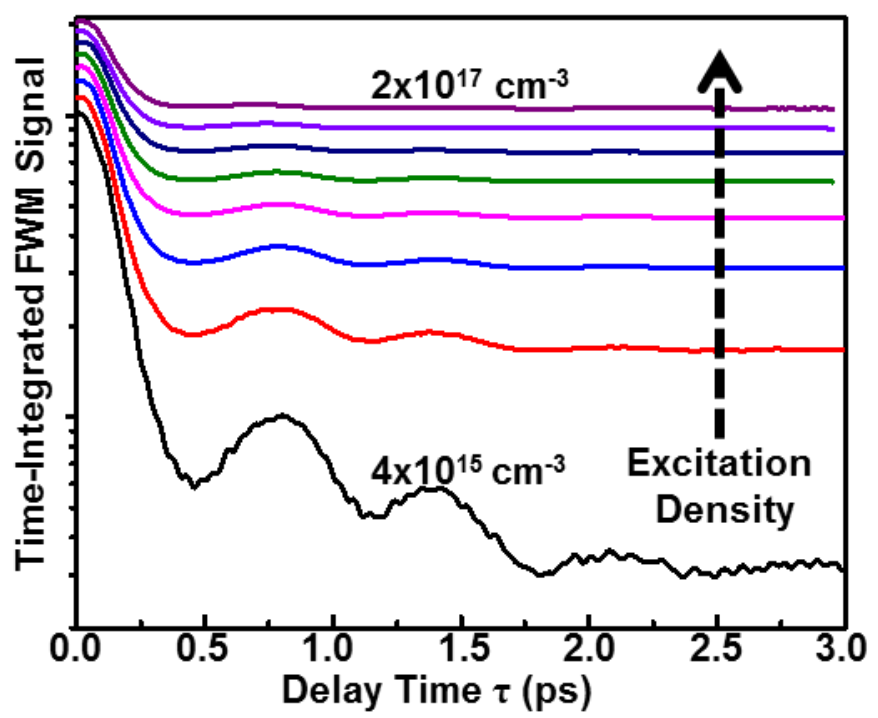

Figure 52 Time integrated FWM intensity as a function of time delay $\tau$ at increasing excitation density.

The $\tau$-dependence of the time-integrated FWM signal shows a decrease in the quantum beating between the heavy-hole and light-hole excitons, see Figure 52. Generally, at higher excitation density, more excited carriers are present which result in more $\mathrm{MBI}$. It is clear that increasing $\mathrm{MBI}$ causes a decrease in beat amplitude as well as a slight increase in beat frequency, which is clearer at high densities $\left(\sim 10^{17}\right)$.

The beat period is $648 \mathrm{fs}$ corresponding to a beat frequency of $\sim 1.5 \mathrm{THz}$ and an energy of $6.4 \mathrm{meV}$ which is exactly the splitting between the two exciton states. At low excitation density where the $l$ and $h$ peaks differ only by about 2 , strong quantum beats are observed in the TIFWM signal. As the excitation density is increased the $l$ peak saturates, the EIS shifts the peak energies, and dephasing from EID increases (weakening the coupling), as observed in Figure 51 (c), which manifest as weaker quantum beats, an increased beat frequency as a result of decreased splitting, and an overall faster decay time of the entire response. 


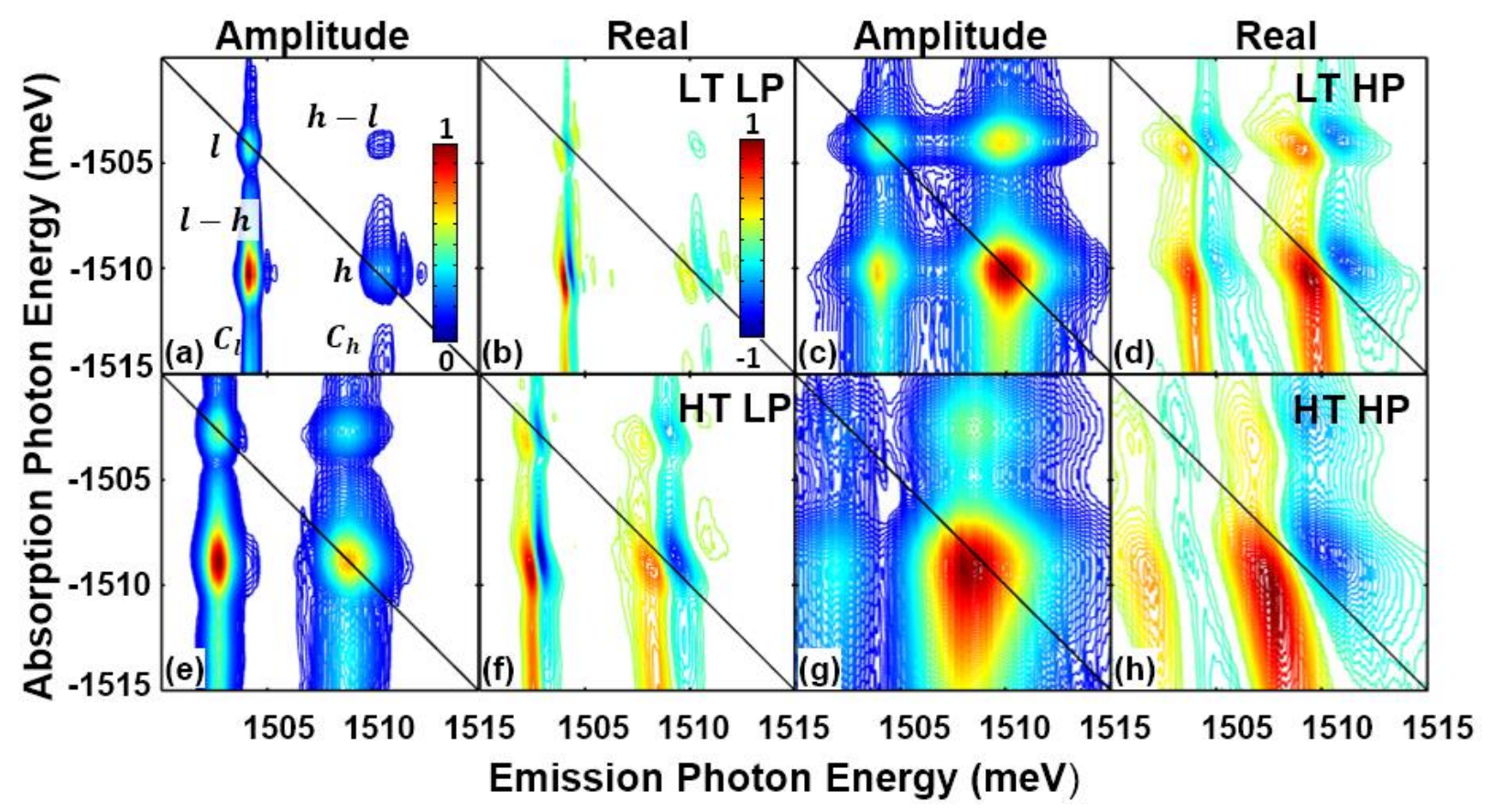

Figure 53 Amplitude (a, c, e, g) and real (b, d, f, h) MDCS spectra at low (a, b, e, f) and high (c, d, $\mathrm{g}$, h) excitation density and low (a-d) and high (e-h) temperature. LT and HT indicate low (6 K) and high $(40 \mathrm{~K})$ temperature, respectively, and LP (low power) and HP (high power) indicate low power excitation generating low $\left(4 \times 10^{15} \mathrm{~cm}^{-3}\right)$ and high $\left(2 \times 10^{18} \mathrm{~cm}^{-3}\right)$ excitation densities, respectively.

Two-dimensional MDCS spectra as functions of temperature and excitation density are shown in Figure 53. The low temperature and low excitation density scenario. Figure 53(a, b) is the same as discussed earlier in section 4.4 of the high strain sample. At high power Figure 53(c, d), EID widens all peaks and the real spectra reflects that EID is still strong with widened dispersive line shapes. The coherences, $l-h$ and $h-l$ become more symmetric and the saturation of the $l$ peak with respect to $h$ is also seen in the drop in relative intensity. As the temperature is raised from $6 \mathrm{~K}$ to $40 \mathrm{~K}$ the bandgap and states redshift, by $\sim 1.5 \mathrm{meV}$, as a result of the strain contrast between the substrate and GaAs lessening as well as the GaAs expanding from increased phonon presence. This shift can be seen in the diagonal shift of Figure 53(e-h) compared to (a-d). 


\begin{tabular}{|c|c|c|}
\hline \multicolumn{3}{|c|}{ Equation } \\
\hline & Value & Value \\
\hline & $\underline{3}=A+B x+C x^{2}+D x^{3}$ \\
\hline Parameter & $\underline{\text { Peak } l}$ & $\underline{\text { Peak } h}$ \\
\hline$A$ & 0.0 & 0.0 \\
\hline$B$ & $9.9 \times 10^{-19}$ & $2.6 \times 10^{-36}$ \\
\hline$C$ & $-2.9 \times 10^{-36}$ & $-4.2 \times 10^{-53}$ \\
\hline D & $3.2 \times 10^{-54}$ & $-9.6 \times 10-54$ \\
\hline
\end{tabular}

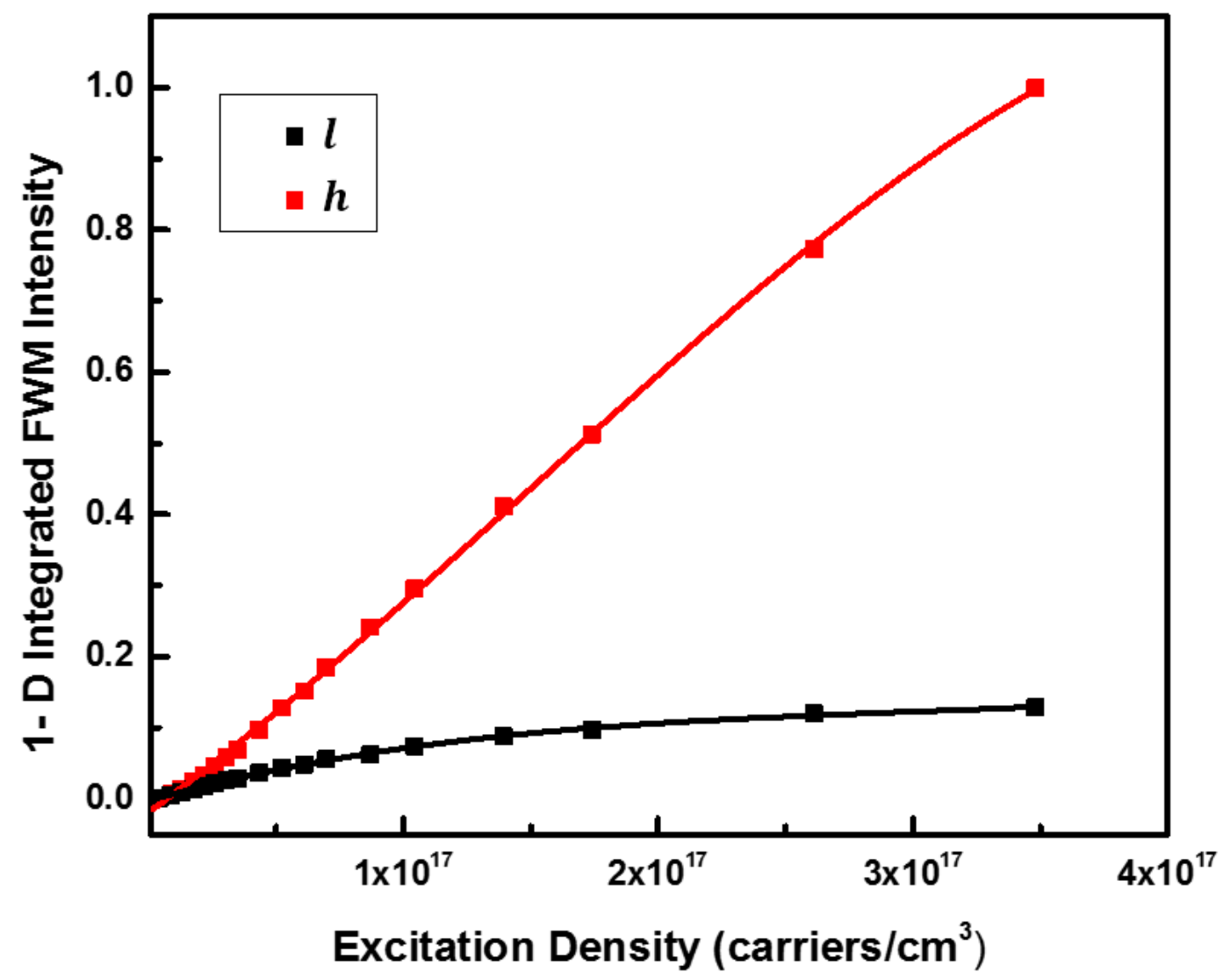

Figure 54 Integrated FWM peaks from one-dimensional spectra

Figure 54 shows integrated FWM peaks extracted from SR-FWM spectra and the fits in the able above. The one-dimensional data shows a clear rolling over, saturative effect due to phase space filling and EID. [21] The heavy hole peak has a stronger initial quadratic curve that follows the $\mathrm{N}^{2}$ dependence of the increase of oscillators, whereas the $l$ peak begins to show 
saturative effects at lower excitation densities, which possibly indicates a lower density of states available for occupation by excited carriers.

Table 4 Information regarding the fits in Figure 55.

\begin{tabular}{|c|c|c|}
\hline \multicolumn{3}{|c|}{ Equation } \\
\hline \multicolumn{3}{|c|}{$y=A+B x+C x^{2}+D x^{3}$} \\
\hline & Value & Value \\
\hline Parameter & (a) Peak $l-h$ & (a) Peak $h-l$ \\
\hline$A$ & 0.41651 & 0.12063 \\
\hline$B$ & $-3.85099 E-18$ & $2.29034 \mathrm{E}-18$ \\
\hline C & 2.83741E-35 & $-1.68445 E-35$ \\
\hline \multirow[t]{2}{*}{ D } & $-7.10713 E-53$ & $4.09762 E-53$ \\
\hline & (b) Peak $l$ & (b) Peak $h$ \\
\hline$A$ & 0.17978 & 0.28308 \\
\hline$B$ & $-5.5854 \mathrm{E}-19$ & 2.11919E-18 \\
\hline C & 3.84177E-36 & $-1.53713 E-35$ \\
\hline \multirow[t]{2}{*}{ D } & $-9.79198 E-54$ & $3.9887 E-53$ \\
\hline & (c) Peak $l$ & (c) Peak $h$ \\
\hline$A$ & 0.52848 & 1.23268 \\
\hline$B$ & $9.60282 \mathrm{E}-19$ & 2.77461E-18 \\
\hline $\mathrm{C}$ & $-8.89469 E-36$ & $-4.09064 E-35$ \\
\hline D & $8.31306 E-53$ & $2.11504 \mathrm{E}-52$ \\
\hline
\end{tabular}




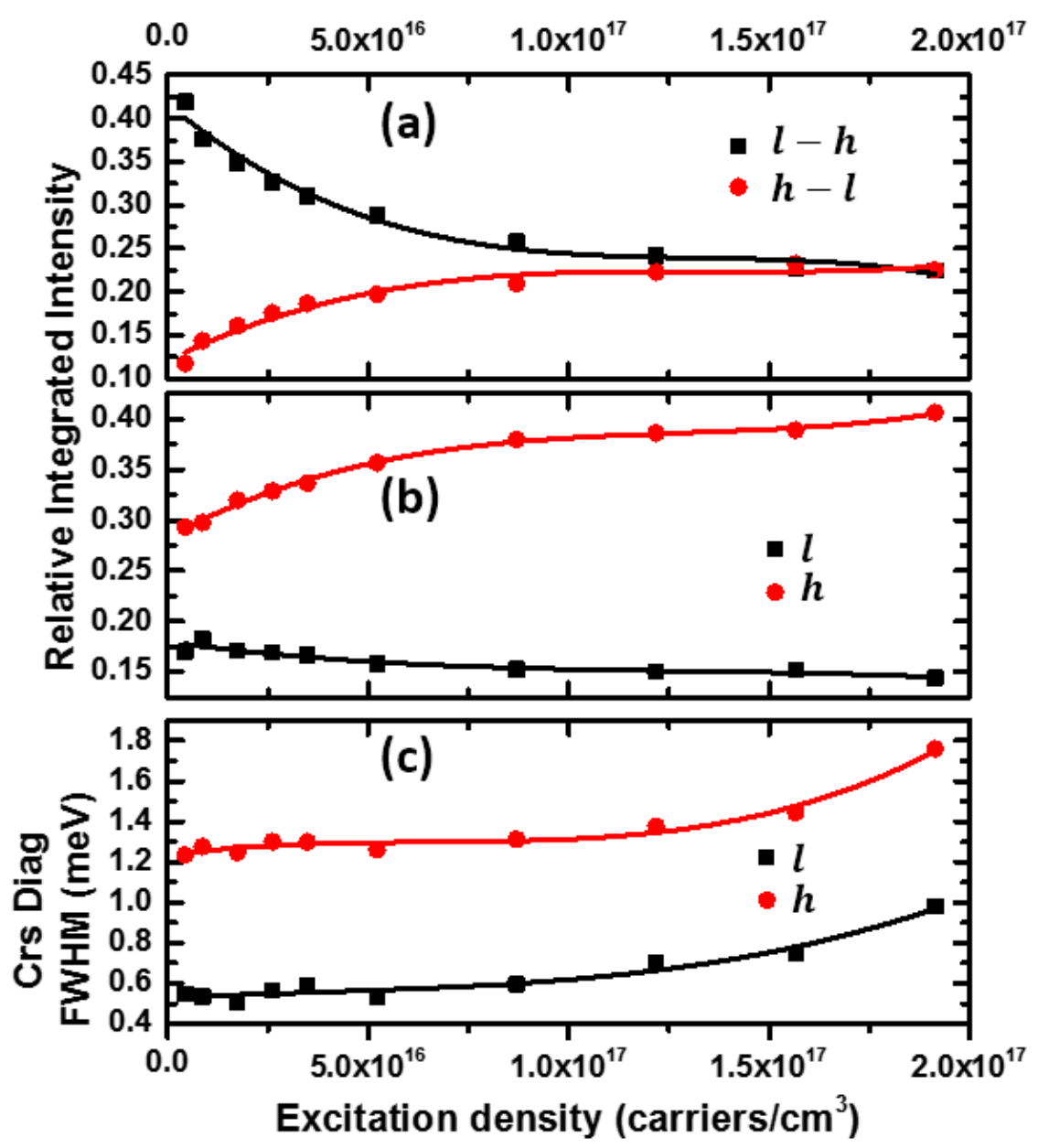

Figure 55 Fits and raw data of (a) integrated off-diagonal coherences (b) integrated diagonal coherences and (c) the diagonal FWHM as functions of excitation density.

Figure 55 shows the relative integrated intensity of the off-diagonal peaks in (a), the diagonal peaks in (b), and the cross diagonal FWHM as function of excitation density (c). All of which were extracted from 2D spectra. The data is fit with a polynomial, shown in Table 4 along with the fitted parameters, as this excitation density dependent data could have contribution from different order nonlinearities. One can see that the asymmetry of the diagonal peaks $l$ and $h$ increases as the off-diagonal peaks $l-h$ and $h-l$ decrease. This indicates an inverse relationship between the intra-action and inter-action, which was not possible to observe in the 1D data as a result of increased $\mathrm{MBI}$ and phase space filling. Since the one-dimensional data 
convolves what would be four peaks into two peaks along the emission axis, the off-diagonal coherences must be at least partially responsible for these effects. This is consistent with the increasing EID as a function of excitation density, as seen in the TI SRFWM vs time delay. At higher excitation intensity, the diagonal coherences maintain roughly the same asymmetry.

Table 5 Information regarding fits in Figure 56

\begin{tabular}{|c|c|c|}
\hline \multicolumn{3}{|c|}{$y=y_{0}+A e^{\frac{x}{B}}$} \\
\hline \multicolumn{3}{|c|}{ Value } \\
\hline Parameter & (a) Peak $l$ & Value \\
\hline$y_{0}$ & -.7812 & (a) Peak $h$ \\
\hline$A$ & 1.9381 & .1358 \\
\hline$B$ & .0642 & 1.0956 \\
\hline & (b) $\underline{l \text { Ln(Crs Diag FWHM) }}$ & (b) $\underline{h \text { Ln(Crs Diag FWHM) }}$ \\
\hline$y_{0}$ & -1.7215 & -1.2466 \\
\hline$A$ & -.6220 & .6847 \\
\hline$B$ & .0232 & .0266 \\
\hline
\end{tabular}




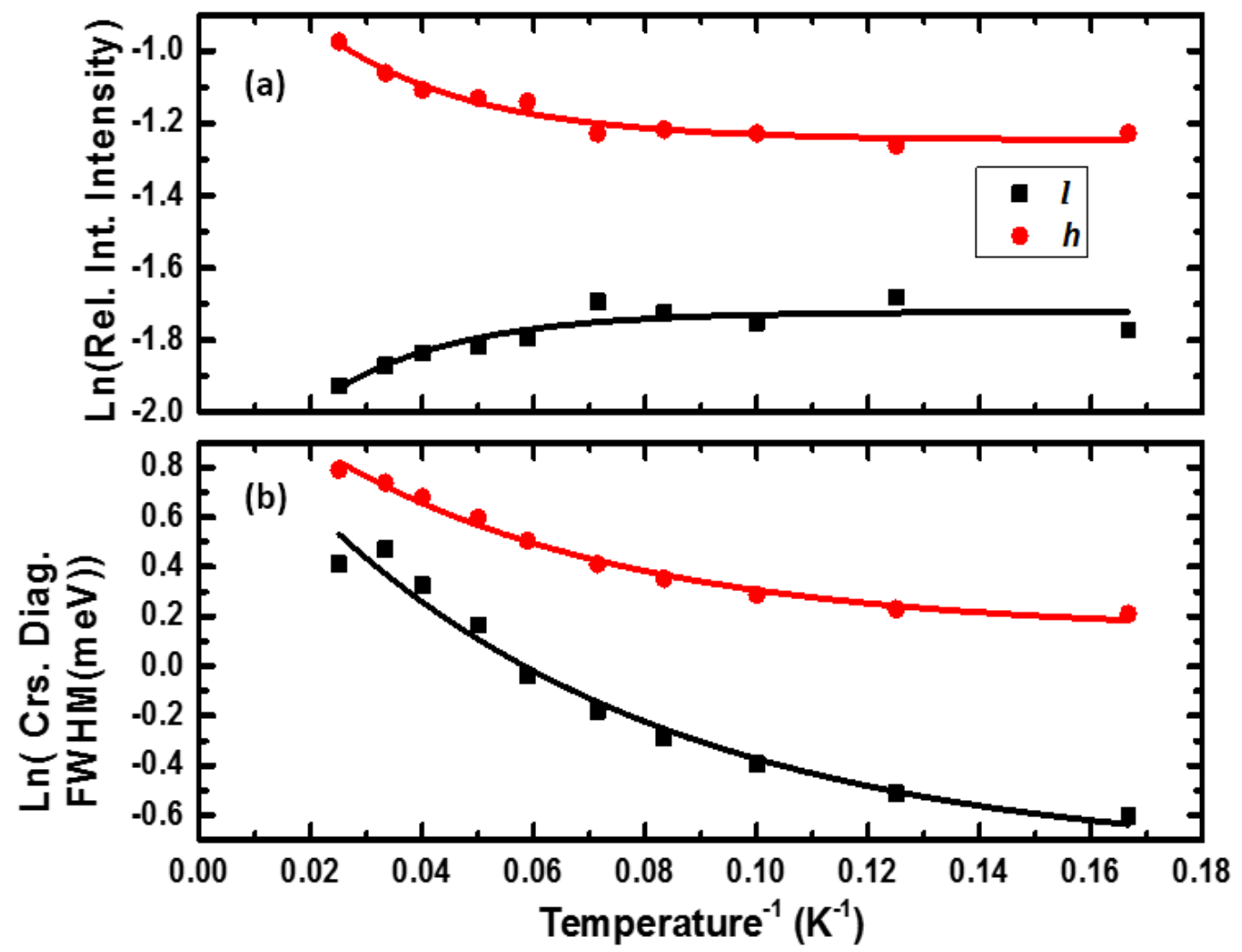

Figure 56 Fitted and raw data of the (a) diagonal coherences and (b) the cross diagonal FWHM of the diagonal coherences as a function of (inverse) temperature

Figure 56 (a) shows the extracted relative intensity of the diagonal peaks and (b) their cross diagonal FWHM as a function of inverse temperature. As temperature increases from 6 to $40 \mathrm{~K}$, the thermal energy in the system increases from $\sim .09 \mathrm{meV}$ to $25.7 \mathrm{meV}$ according to $k_{B} T$. The increase in homogeneous dephasing is due to a thermal population of phonons $[130,176]$, which is now considerable compared to the binding energy of the $1 \mathrm{~s}$ exciton which is $\sim 4 \mathrm{meV}$ and has been shows to significantly increase all linewidths as temperature increases in quantum dot systems with weakly bound excitons $[63,188]$. The end result is that the cross diagonal linewidth increases as a function of temperature and the overall intensity of the diagonal coherences drops, while the asymmetry between them decreases.

\subsection{Non-radiative Coherences}

Zero quantum $S_{I}$ data shows very little if any nonradiative, Raman-like coupling between the heavy and light hole states, see Figure 57. Indicated there is effectively no dephasing 
occurring during the population mixing time $\mathrm{T}$ other than the comparatively slow population relaxation.

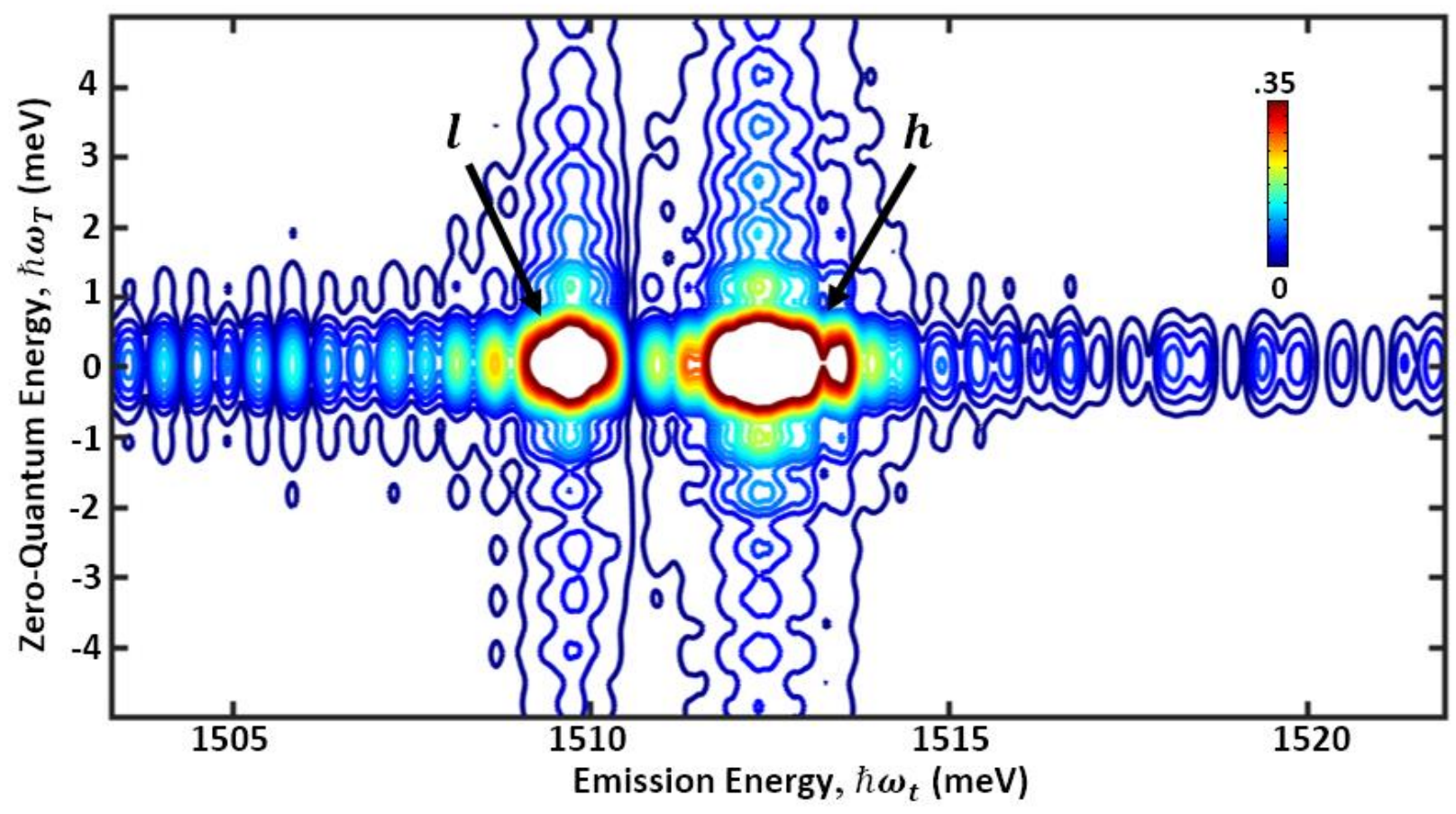

Figure 57 Zero-quantum scan of the strained bulk GaAs system, showing no discernable Ramanlike peaks.

\subsection{Two-quantum Contributions}

Two-quantum scans on the low-strain sample reveal biexciton contributions not discernable in the one-quantum data. Under collinear excitation, Figure 58(a), $h h, l l$, and mixed biexciton $(l h, h l)$ species are present. The higher two-quantum energy streaks are two to continuum absorption between unbound four-particle pairs. The two $B_{\llcorner}$peaks result from the solid square and rectangle diagrams of Figure 14 and communicate whether the final radiating coherence is between a biexciton and an exciton or an exciton and the ground state. Under cocircular excitation, Figure 58 (b), heavy and light hole biexcitons cannot occur, but rather only mixed biexciton states [121]. Correlated (but unbound) heavy hole excitons $(h-h)$ appear in place of $\mathrm{B}_{\mathrm{H}}$. 


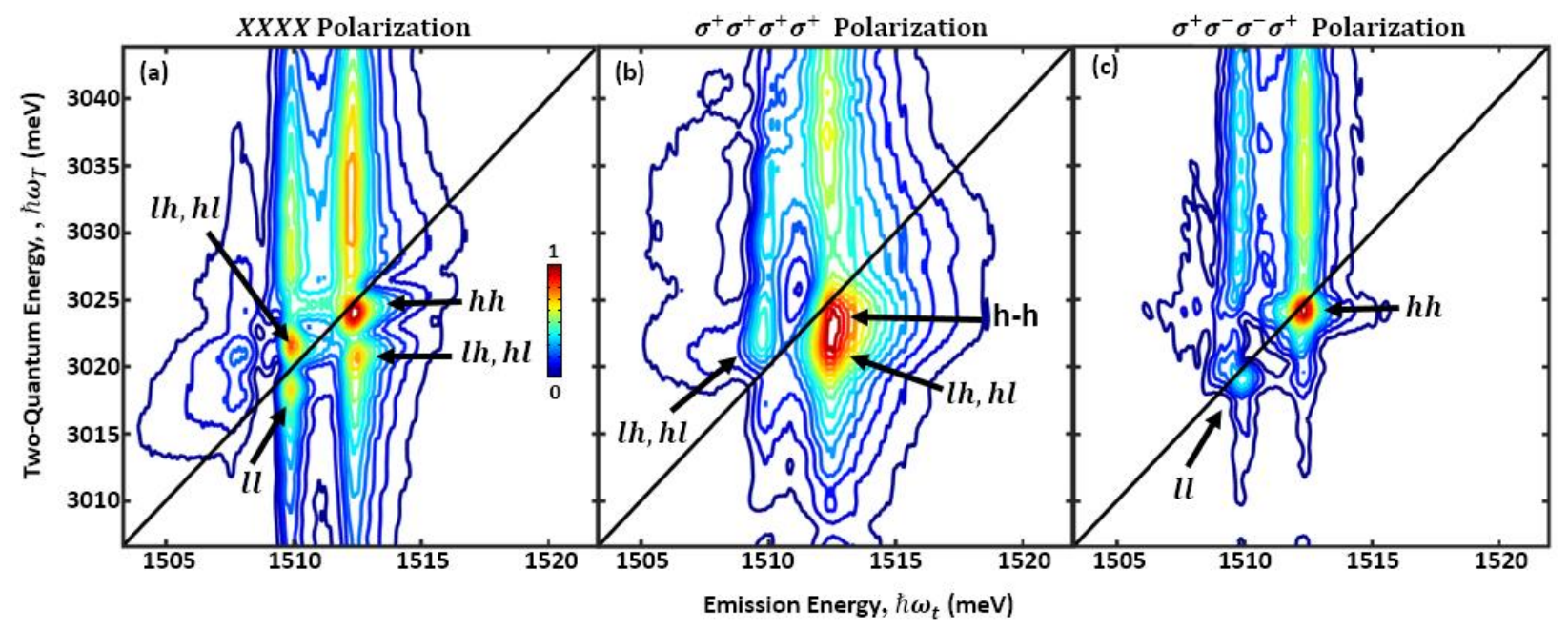

Figure 58 Two-quantum, $S_{I I I}$ scans at (a) collinear (XXXX) (b) cocircular $\left(\sigma^{+} \sigma^{+} \sigma^{+} \sigma^{+}\right)$and (c) crosscircular $\left(\sigma^{+} \sigma^{-} \sigma^{-} \sigma^{+}\right)$polarizations

Conversely, under crosscircular excitation Figure 58(c), single species biexcitons may occur but not mixed. Therefore, we see only the clear $h h$ and $l l$ peaks. The two weak peaks in the collinear data, offset from the BL emission energy by $\sim 2 \mathrm{meV}$, are possibly EID mediated coupling between $l$ and $l h, h l$, as they do not appear in the circular data.

\subsection{Conclusions from Study of Strained Bulk GaAs Excitons}

Two samples of bulk GaAs attached to sapphire disks experience differing amounts of biaxial tensile strain due to their relaxation over differing volumes upon cooling. Strain splits and shifts the observed $\mathrm{HH}$ and $\mathrm{LH}$ excitons in optical absorption and 2DCS, which is used to measure the coherent response. Increasing the strain has several effects on the exciton resonances: (i) the separation between the excitons increases; (ii) the strength of off-diagonal coherences become more asymmetric; (iii) the difference between the $h$ and $l$ homogenous linewidths increases as a result of greater energy separation; and (iv) the inhomogeneous broadening increases due to an increase in the strain relaxation gradient in the growth direction for thinner samples.

By focusing on bulk layers that are significantly thick compared to the excitonic Bohr radius, the influence of strain on the exciton and biexciton resonances is probed in the absence of quantum confinement. The application of 2DCS extracts both the average effective strain and 
distribution in effective strain experienced by the excitons throughout the excitation volume. Using collinear polarization, the one-quantum excitonic features are enhanced by many-body interactions and dominate the spectra. The spectral weight of the excitons become sensitive to the degree of separation induced by the strain. For small $h / l$ separation, the spectral weight of the 2DCS features is more akin to an atomic vapor than the usual semiconductor nanostructure response. Alternatively, increasing strain allows the spectra to begin exhibiting the effects associated with increased excitation-induced MBI.

It is also shown that biexcitons are more easily observed in structures with large average strain due to the large $h / l$ splitting relative to the biexciton binding energy. For cross-linear polarization, no population grating is created and, as a result, signal contributions associated with $\mathrm{MBI}$ are suppressed, allowing biexciton contributions to be revealed. Biexcitons are a manifestation of few-body interactions and are also expected to be more prevalent in samples where the $h$ and $l$ states are distinct. In addition, the first evidence of a mixed biexciton observed by one-quantum 2DCS is presented.

Overall, these results show the sensitivity of 2DCS to strain, excitation density, and thermal energy through interrogation of the coherent response and effects on the spectral line shape and spectral weight of all visible features. In particular, the analysis of the inhomogeneous broadening of bulk excitons reveals strain gradients that are stronger in thinner GaAs layers. This significant change in the coherent response invites studies where the strain can be controlled while measuring EID, EIS and biexciton formation. [69] Temperature and excitation density underline the sensitivity of dephasing and the coherent four-wave mixing response to the microscopic environment. Marked widening of all peaks occurs as temperature and excitation density increase, reflecting the increased dephasing from EID and high energy phonons. Information learned here is part of the growing series of studies aimed at using sophisticated optical spectroscopy to unravel the electronic environment inside micro and nanostructures. 


\section{Chapter 5 Quantum Microcavity Polaritons-Results}

In this work, normal-mode splitting of a semiconductor microcavity and the associated exciton-polariton branches are studied using rephasing and nonrephasing $S_{I-I I I}$ type 2DCS scans as functions of detuning and excitation polarization.

\subsection{Detuning Dependence}

Despite the extensive fundamental and applied studies of microcavity exciton-polaritons, the anticrossing has not been systematically mapped using MDCS previously. The study is performed over a range of energy detuning $\left(\Delta=E_{C}-E_{X}\right)$ near the anticrossing, where $E_{C}$ and $E_{X}$ correspond the cavity-mode and exciton-mode energies, respectively. The detuning dependence of spectral features, related to intra-action (diagonal features) of and interaction (off-diagonal features) between polariton branches, informs us about the coupling between the cavity and excitonic or biexcitonic modes. For example, a contribution from a bound biexciton is isolated at negative detuning, which is convolved with the off-diagonal features at positive detuning and strongly modulates their relative amplitudes. 


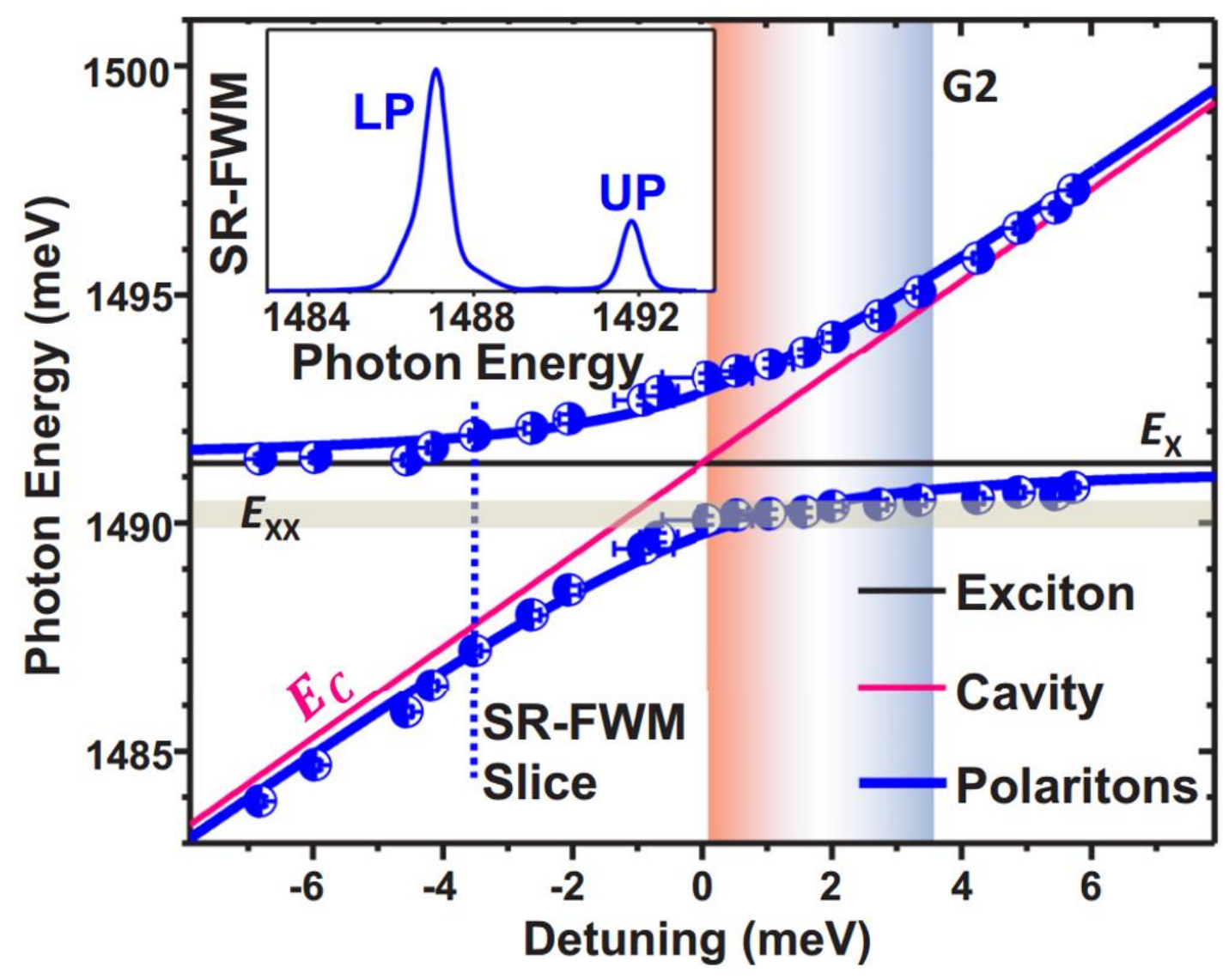

Figure 59 Detuning dependence of the features observed in SR-FWM. A typical FWM spectrum is shown in the inset, from which the center energies of the lower (LP) and upper (UP) polaritons are extracted for the body of the figure. Solid lines model the vacuum Rabi splitting, the bare exciton energy, and the detuning for each spectrum. Also shown are the expected energy of the bound biexciton $E_{X X}$ and the detuning range of the $\mathrm{G} 2$ profile discussed in Figure 61. Modified from reference [43]. 
Spectrally resolved FWM is acquired as a precursor to performing 2DCS with $\tau=0 \mathrm{fs}$ and $T=100 \mathrm{fs}$. The inset of Figure 59 shows a typical spectrum, revealing resonances associated with the lower (LP) and upper (UP) polariton branches. The remainder of Figure 59 shows the spectral positions extracted from spectra measured at different positions on the sample.

The detuning $\Delta$ is determined for each spectrum by modeling the data using

$$
E_{U P / L P}=\frac{1}{2}\left(2 E_{X}+\Delta \pm \sqrt{\Delta^{2}+E_{R}^{2}}\right)
$$

where $E_{R}$ is the Rabi splitting, a measure of the coupling between the optical field and the excitons. It is found that $E_{R}=3.1 \pm 0.1 \mathrm{meV}$ and $E_{X}=1491.3 \pm 0.03 \mathrm{meV}$, which both agree well with results on similar structures $[95,158,189]$. The inset spectrum is then indicated as a dashed vertical line at $\Delta \cong-3.75 \mathrm{meV}$. Microcavities are sensitive to the angle of incidence, since the dispersion varies with the in-plane wave vector. Here the external angle of incidence for each beam is approximately near normal, ( $\sim 7^{\circ}$ deviation) and the axis of the box of pulses is at normal incidence. Each beam couples to slightly different polariton states in momentum space, which may lift the measured energy and lower the overall signal strength. However, close to zero detuning, the FWM emission requires attenuation of at least ND2.5, indicating minimal momentum-related signal reduction and excitation within the parabolic region of the in-plane momentum [190].

Figure 60 shows the absolute field amplitude of the rephasing (bottom row) and nonrephasing (top row) 2DCS results for a range of detuning values from (a) $\Delta=-5 \mathrm{meV}$ to (e) $+4.5 \mathrm{meV}$. Excitation is performed with a mixing time $T=100 \mathrm{fs}$ and collinear polarization (XXXX), where the notation corresponds to the polarization state of the three pump pulses and the emission. Each panel is normalized to the strongest peak for presentation. At each value of $\Delta$ the laser spectrum is overlaid with the nonrephasing spectrum, illustrating that the two resonances are excited equally in each case. This is important for careful comparison of the relative amplitudes of each feature as a function of detuning. Due to the transmission geometry, strong absorption of the tracer beam prevents experimental determination of the global phase using 
all-optical methods or via spectrally resolved transient absorption [54]. Hence, only amplitude spectra are shown.

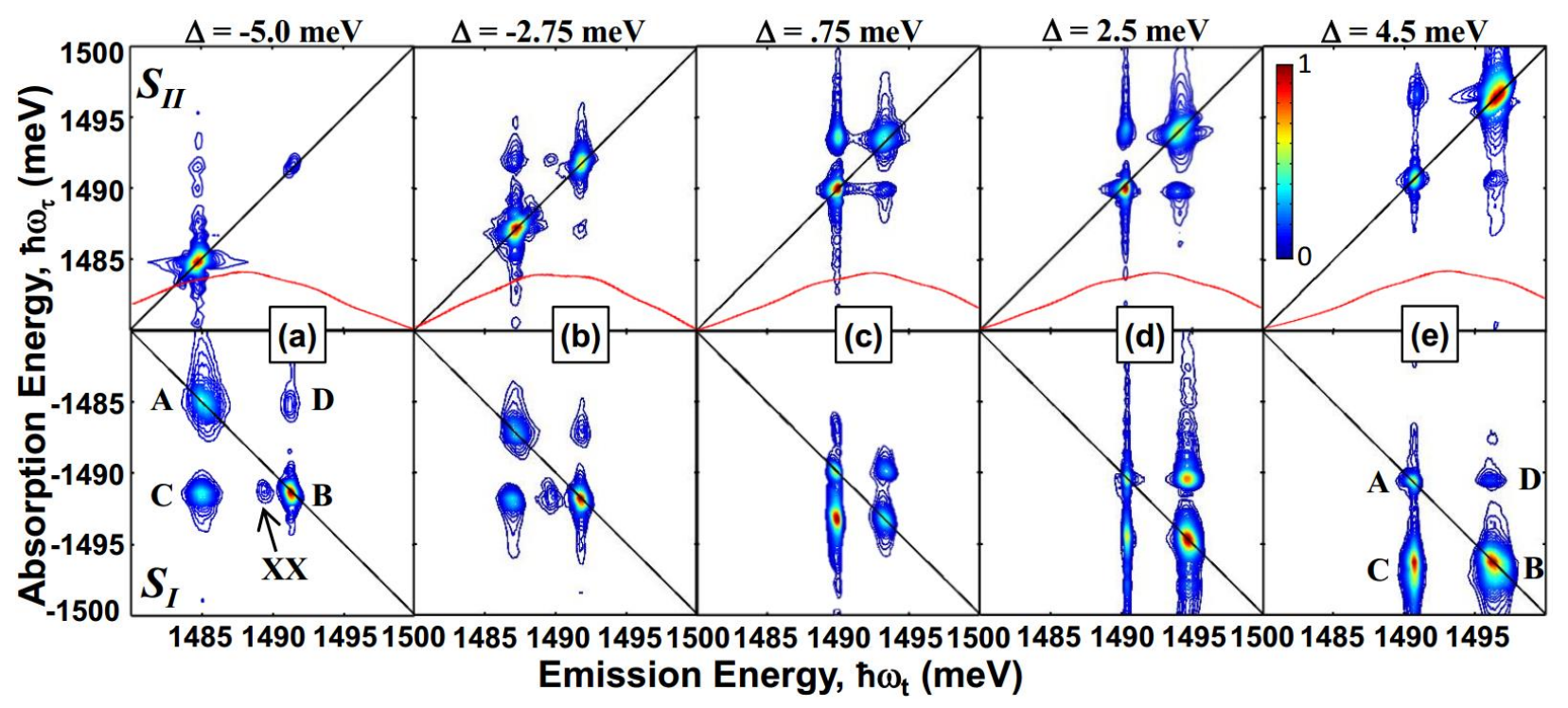

Figure 60 Rephasing $S_{I}$ (bottom row) and nonrephasing $S_{I I}$ (top row) two-dimensional coherent spectra for the labeled range of detuning. Also shown is the laser excitation spectrum for each excitation position. Diagonal intra-action features are $A$ and $B$, and off-diagonal interaction features are $C$ and $D$. Negative detuning also shows a biexciton feature XX. Reproduced from reference [43].

In Figure 60 (a) the $C$-like mode is the low-energy feature, denoted $\mathrm{A}$, and is broader than the higher-energy $X$-like mode, denoted B. From analysis of the line shapes the cross-diagonal width is dominated by the homogeneous linewidths [62] of intra-action features $A$ and $B$, yielding values of $\gamma_{L P}=0.35 \mathrm{meV}$ and $\gamma_{U P}=0.14 \mathrm{meV}$, respectively. In comparison, the diagonal linewidths, which can be dominated by inhomogeneous broadening, are only slightly wider in each case: $\sigma_{L P}=0.38 \mathrm{meV}$ and $\sigma_{U P}=0.17 \mathrm{meV}$. In addition to the diagonal intra-action features, two off-diagonal interaction features $C$ and $D$ are observed, which are due to coherent coupling between the A and B features [21].

Increasing $\Delta$ shifts all spectral features toward higher energy. The separation between the $\mathrm{A}$ and $\mathrm{B}$ modes (projected onto the emission axis) is $\sim 7 \mathrm{meV}$ in (a), decreases as $\Delta$ tends to zero, becoming $3.4 \mathrm{meV}$ in (c), and increases again to $5.6 \mathrm{meV}$ in (e). Close to zero detuning $(\Delta=$ $0.75 \mathrm{meV}$ ), the homogeneous linewidths of $\mathrm{A}$ and B in the $S_{I}$ spectra are $\gamma_{L P}=0.12 \mathrm{meV}$ and $\gamma_{U P}=0.18 \mathrm{meV}$, respectively. The properties are nearly identical and $C$-like and $X$-like 
characteristics are no longer distinguishable, since it is expected that the observed linewidths should become identical [191]. For larger positive detuning the homogeneous linewidths of $A$ and $\mathrm{B}$ are $\gamma_{L P}=0.14 \mathrm{meV}$ and $\gamma_{U P}=0.24 \mathrm{meV}$, respectively. The $C$-like and $X$-like characteristics are once again distinguishable, but are not quite the same as for negative detuning. As expected from analysis of the Hopfield coefficients [93], the modes switch and the $C$-like mode is now the upper polariton B. The mode switch is consistent with results where the mirror reflectivity results in a narrower cavity than bare exciton linewidth [191].

Collinear polarization in 2DCS allows for excitation to the biexciton $(X X)$ states. $[66,111,112]$ For bound biexcitons, binding energy acts to shift the $X X$ feature laterally from the $X$-like mode in the emission energy $\omega t$. This feature is only observed for negative detuning, from which the $X X$ binding energy is determined to be $\sim 1.88 \mathrm{meV}$. The biexciton follows the expected excitation-density dependence and is suppressed for co-circular polarization (data not shown). Its cross-diagonal linewidth is almost identical to that for the $X$ like mode, which is expected because the quantum pathway that creates the $X X$ feature is a twostep excitation via the exciton. Hence, the linewidth projected on the absorption energy $-\omega \tau$ should be identical to that for the exciton. The linewidth projection onto $\omega t$ may be a little wider (tilting the feature away from the diagonal), depending on the degree of correlation of the exciton and biexciton states $[182,192]$. In this case, the exciton and its biexciton are parallel and are highly correlated.

Nonrephasing $S_{I I}$ are presented for comparison, showing very similar results to $S_{I}$ spectra across the entire detuning range. $S_{I I}$ spectra typically have slightly weaker off-diagonal features, as is observed here. Otherwise, the two diagonal intra-action, two off-diagonal interaction, and biexciton features are all observed as discussed above.

Figure 61 shows the integrated amplitude versus detuning for the four main polaritonic features A through D. In each case, a small area around each feature is integrated. Figure 61 (a) shows the total integrated amplitude for a $\sim 12 \mathrm{meV}$ detuning range and is normalized to the highest emission strength at $\Delta=0 \mathrm{meV}$. Significant enhancement of the exciton-polariton transition are observed due to normal-mode coupling [163], as the integrated amplitude 
increases and peak close to zero detuning. The peak shape of extracted total amplitude is asymmetric in detuning [193] and can be fit with two Gaussian line shapes: $G 1$ is centered at zero detuning and $G 2$ is centered at $1.88 \mathrm{meV}$, corresponding to the biexciton binding energy determined from negatively detuned spectra. Fitted full widths at half maximum of $G 1$ and $G 2$ are $\sim 3.39$ and $\sim 3.65 \mathrm{meV}$, respectively. The sum of $G 1$ and $G 2$ well represent the -dependence peak shape.

Figure 61 (b) and (c) show $\Delta$ dependence of the relative amplitude for the individual diagonal intra-action and off-diagonal interaction spectral features. From each spectrum the individual features' integrated amplitudes are extracted and normalized to the total integrated amplitude. At zero detuning the relative amplitudes of the diagonal intra-action features are identical, showing that normal-mode coupling leads to enhancement and equalization of the UP and LP branches. Away from zero detuning the higher-energy diagonal feature $B$ is always stronger and the overall amplitude of the diagonal features drop at small positive detuning. In contrast, the relative integrated amplitude of the off-diagonal features is smaller at negative detuning and oscillates for positive detuning. The oscillation position is consistent with the position of the small $G 2$ peak from Figure 61(a). In this range, the amplitude of peak $\mathrm{C}$ increases rapidly, corresponding to the increasing slope of $G 2$, and decreases rapidly to become smaller than $\mathrm{D}$, corresponding to the decreasing slope of $G 2$. Both the width of $G 2$ and the range of $\Delta$ where the oscillations occur agree well with the width of the LP branch passing through the bound biexciton, see Figure 59 .

The solid lines in Figure 61 (b) and (c) model the relative amplitudes of the diagonal and off-diagonal features based on the following parameters. First, amplitude offsets for each feature are selected from negatively detuned spectra, yielding $25 \%, 43 \%, 10 \%$, and $12 \%$, for $A, B, C$, and D, respectively. 


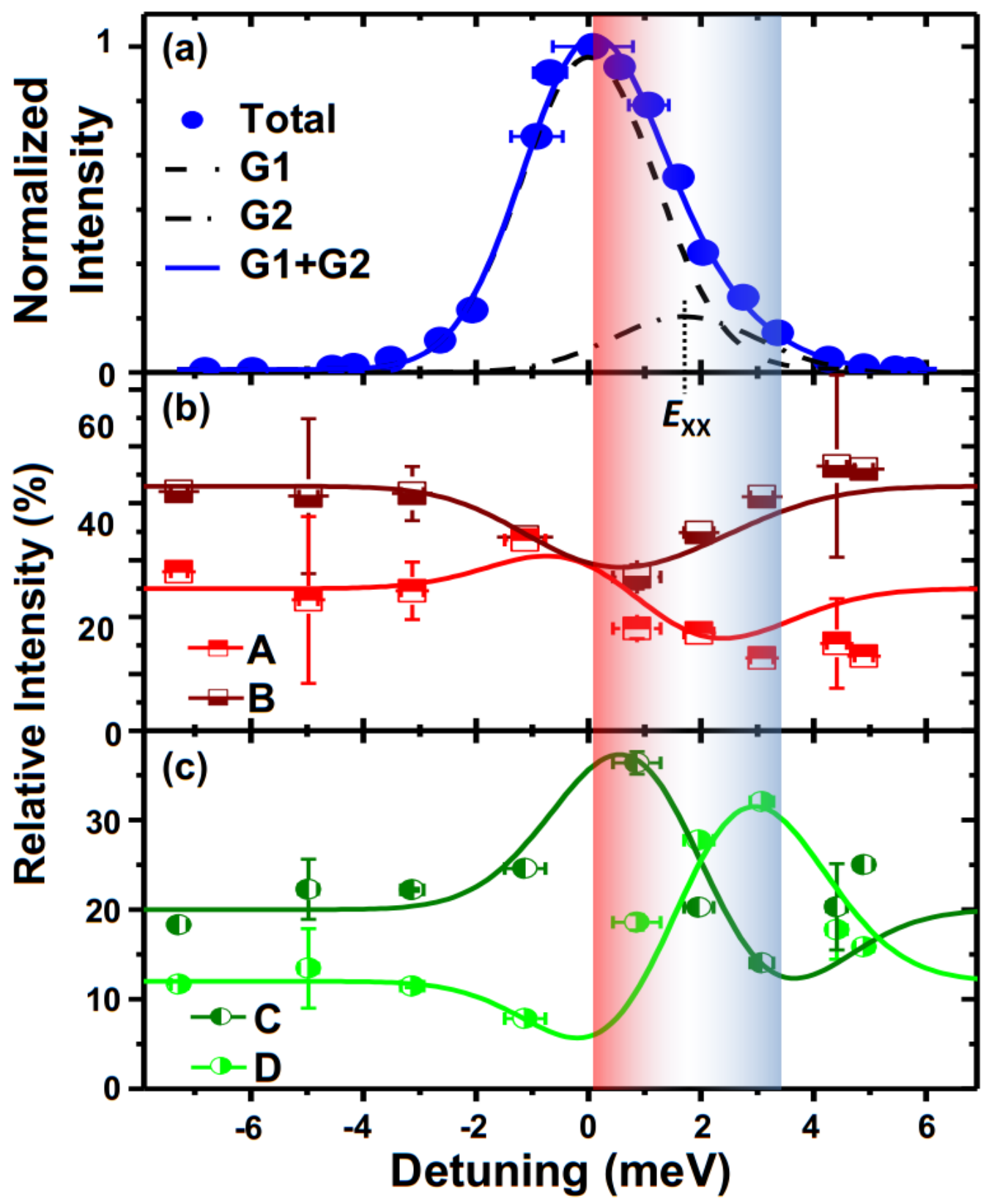

Figure 61 Detuning dependence of the features in the rephasing 2DCS. (a) Shows the normalized amplitude of the entire spectrum, with best fits based on two Gaussian profiles G1 and G2. (b) Shows the relative amplitude of the diagonal features A and B. (c) Shows the relative amplitude of the off-diagonal features $C$ and $D$. The solid lines in (b) and (c) model the effect of the two resonances parametrized by the $\mathrm{G} 1$ and $\mathrm{G} 2$ Gaussian profile in (a). Reproduced from reference [43].

Second, near $\Delta=0 \mathrm{meV}$, the strength of the two diagonal features equalizes, bringing the two modes close in amplitude in the range of the $G 1$ profile. This $9 \%$ deviation is symmetric for each feature. Third, in the region of the $G 2$ profile $\sim 11 \%$ of the spectral weight is transferred 
from both diagonal features to the off-diagonal features, most likely due to additional manyparticle interaction terms in the quantum pathways to the biexciton manifold. Fourth, the offdiagonal features experience a $\pm d(G 2) / d \Delta$ modulation in their spectral weight by approximately $\pm 14 \%$, which is most likely due to attraction or repulsion of LP branch as it passes through $X X$. The latter two effects are complementary evidence of the recently observed Feshbach resonance [103]. Feshbach resonances occur when the energy of two free, yet interacting, polaritons is in resonance with the bound molecular excitonic state. Off-diagonal features are the interaction between the LP and UP branches, so that even though the LP branch alone overlaps with the $X X$, both off-diagonal amplitudes are modulated and invert. This result arises from coherent coupling by quantum interference of the polaritons through the shared ground state or by a Raman-like coherence between the excited polaritons [28]. 2DCS sensitivity is revealed, because no splitting is observed associated with the Feshbach resonance in the linear spectra, yet the influence of this LP-XX crossing is clear.

\subsection{Polarization Dependence}

To further investigate the Feshbach and biexcitonic roles, as well as many body interactions, 2DCS spectra were acquired at larger detunings and under different polarization schemes, namely collinear (XXXX), crosslinear (XYYX), cocircular $\left(\sigma^{+} \sigma^{+} \sigma^{+} \sigma^{+}\right)$, and crosscircular $\left(\sigma^{-} \sigma^{-} \sigma^{+} \sigma^{+}\right)$. Note that because of the large number of spectra acquired to provide high detuning resolution, representative subsets of the 2D spectra are presented here.

Figure $62(\mathrm{a})$-(h) shows rephasing spectra taken under the same excitation conditions as that of Figure 60. The labels A-D are the same and upper and lower polariton labels (UP,LP) have been added for clarity. The XX feature from earlier has been relabeled $\mathrm{HH}$ to indicate it is specifically a heavy-hole biexciton, see Figure 62 (b). HH merges into C, see Figure 62 (c), and does not remerge to the left of $A$ where it would appear in Figure 62 (f). Five (possibly seven) additional peaks appear at large positive detuning $(\Delta \geq 5.5 \mathrm{meV}$ ), Figure 62 (f)-(h). Peak E emerges along the diagonal with an energy commensurate with the $2 \mathrm{~s}$ heavy-hole exciton at $1498.2 \mathrm{meV}[143,150]$, see Figure 43. While peak B seems to move further below the diagonal, decrease in strength and but not increase in emission energy with increasing detuning, $B$ is 
actually decaying in amplitude as a biexciton peak is emerging, which is likely a light-hole biexciton owing to the upper branch's proximity to the light-hole exciton state which is known to form biexcitons in GaAs structures $[24,139,194]$ and is the most prominent state in this spectral region besides the polaritons according to Figure 42. It is also a spectrally stationary peak as the $\mathrm{HH}$, with an absorption energy at $\sim 1501 \mathrm{meV}$ and an emission energy at $\sim 1498$ meV This peak is labeled accordingly as LL. The off diagonal (intra-action) peak F indicates weak coupling of the $2 s$ heavy-hole exciton to peak $A$, which is now mostly excitonic in nature at this detuning (also the reason it no longer increases in energy with detuning). Peaks $E$ and $F$ are at least partially polaritonic in nature as both peaks move with increasing detuning and at $6.1 \mathrm{meV}$ two further peaks appear, $\mathrm{G}$ and $\mathrm{H}$ at an emission energy of 1502.809. This energy is close to the both $1 \mathrm{~s}$ light hole state and the lower energy forbidden transition. $G$ is also offset above the diagonal, along the absorption axis by $\sim 1.7 \mathrm{meV}$. This could indicate the state is a scattering state, similar to the open square and triangle Feynman diagrams in Figure 14 where the first interaction with the light fields creates a bound-state coherence followed by signal emission at a higher energy caused by a fast uphill, nonradiative process. Peak $H$ is the coupling of $G$, also to peak $A$.

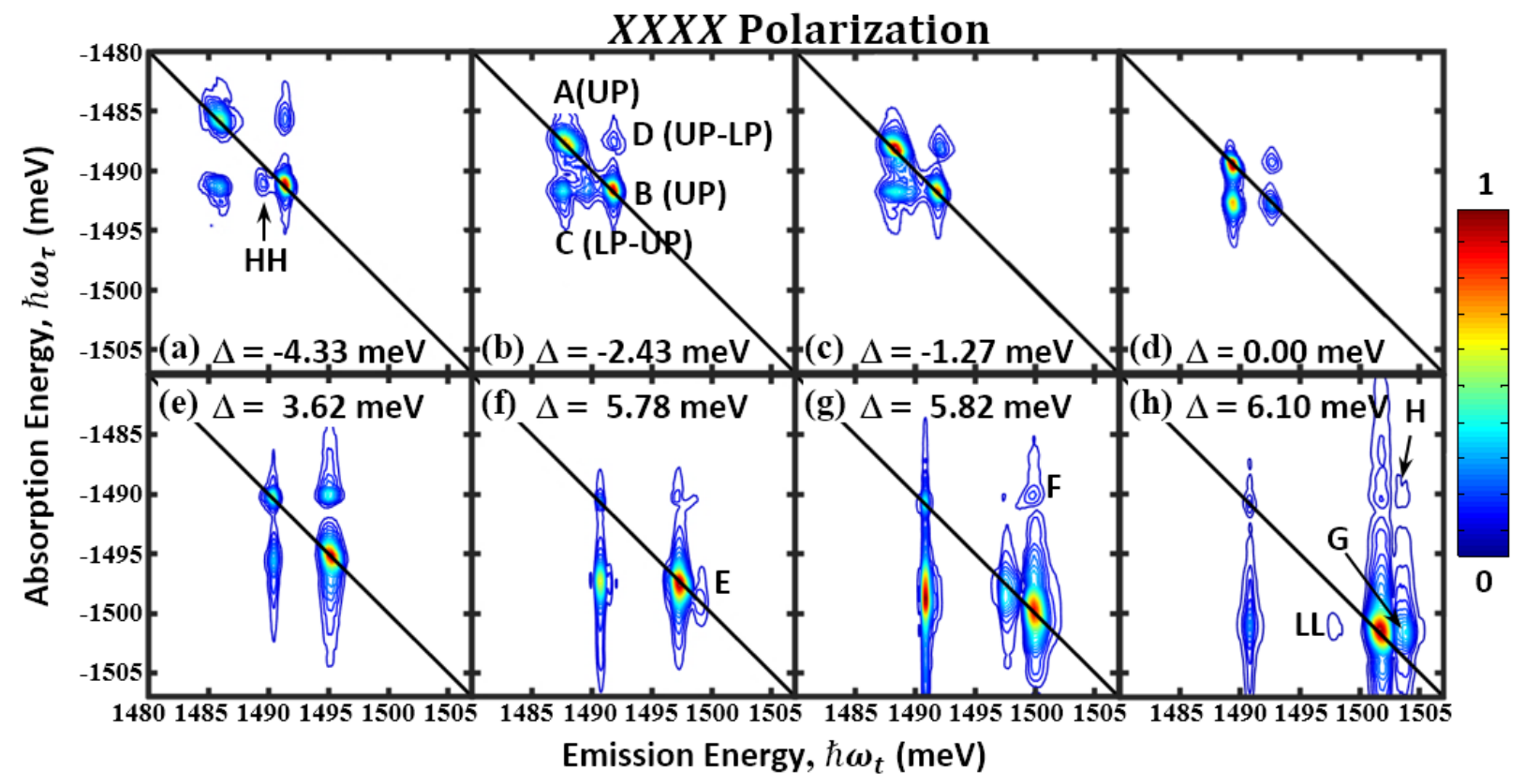

Figure 62 Rephasing $S_{I}$ two-dimensional coherent spectra with collinear excitation for the labeled range of detuning. Diagonal intra-action features are $A(L P)$ and $B(U P)$, and off-diagonal interaction features are C(LP-UP) and D(UP-LP). Negative detuning also shows a heavy hole 
biexciton feature $\mathrm{HH}$. Positive detuning shows an additional diagonal peak $\mathrm{E}$ and three additional off diagonal peaks $\mathrm{F}-\mathrm{H}$.

Figure 63 shows a close-up plot of the peaks near E and G in Figure 62(h). Below peaks E and G, highlighted by the dotted oval, are two very faint possible additional peaks. They could be an off diagonal and a diagonal peak belonging to the 1 s light hole exciton or the dark excitons mentioned in section 3.5.1. If that is the case, $G$ is actually the upper right off-diagonal coherence, completing another set of $2 \times 2$ peaks for a coupled " $V$ " system. To date, forbidden (dark) excitons have not been observed in MDCS spectra. Though because of the cavity coupling, it is possible the peaks are delocalized spectrally from their bare quantum well values. Therefore, making a $1 \mathrm{~s}$ light hole state the likely candidate for the $\mathrm{G}$ transition.

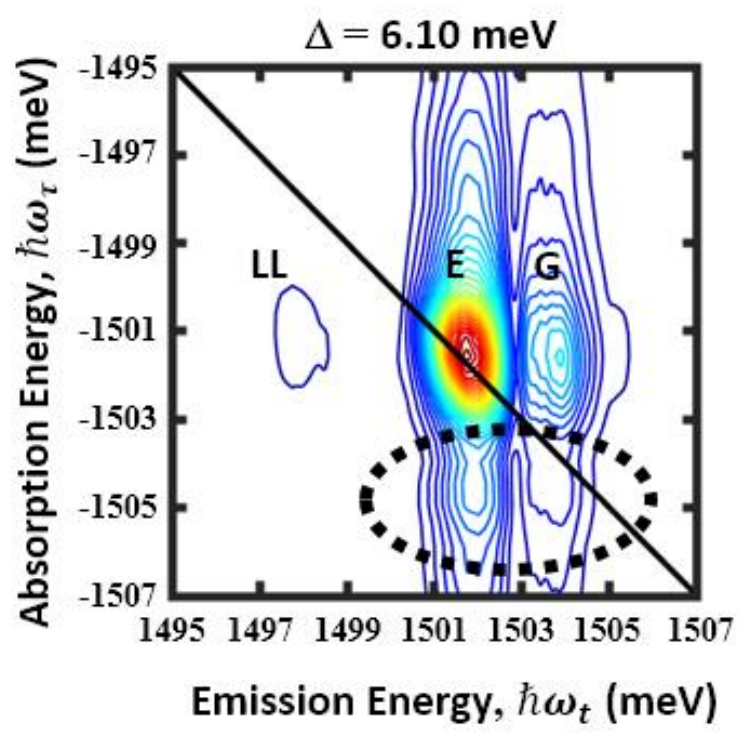

Figure 63 Close-up of the high energy peaks in Figure 62(h).

2DCS spectra acquired under crosslinear polarization (XYYX) are shown in Figure 64 (a)(h). As a result of the $\mathrm{MBI}$ being turned off, there is a reordering of peak intensities and change in linewidths which will be discussed later along with extracted data from all polarizations. Both biexciton features $\mathrm{HH}$ and $\mathrm{LL}$ are present. The $\mathrm{HH}$ biexciton merges into peak $\mathrm{C}$, as $\mathrm{C}$ moves slightly below it, see Figure 64 (b), (c). At $\Delta \approx 3.12 \mathrm{meV}$ detuning, two features to emerge below $\mathrm{C}$ and $\mathrm{B}$, labeled $\mathrm{J}$ and I, respectively. They are seen in Figure 64 (e) at a slightly more positive detuning. The coherences $C$ and $D$ are symmetrically elongated, see Figure 64 (e) and (f). As 
detuning continues to increase, $B$ decreases in amplitude and LL begins to again emerge (spectra not shown between Figure 64 (f) and (g) show the peak clearly). Peak I also strengthens, and at $\Delta \approx 5.3 \mathrm{meV}$ peaks $\mathrm{E}$ and $\mathrm{F}$ again emerge. These are visible in Figure 64 (f). At $\Delta \approx 5.5 \mathrm{meV}$, peak $\mathrm{G}$ again emerges as well as much stronger and well defined peaks below them which were much weaker in the collinear polarized data, see Figure 63. Denote the diagonal peak $L$ and the offdiagonal K. This confirms that peaks E, G, K, L are indeed a sub system of peaks similar to that of a coupled three-state system, which in this case is a coupled $2 \mathrm{~s}$ heavy hole exciton and (the likely) 1s light hole exciton. At slightly larger detuning as in Figure 64 (h), the $2 \times 2$ (square) sub manifold begins to emerge. But before doing so completely, two peaks seem to manifest, labeled $\mathrm{M}$ and $\mathrm{N}$, with an emission energy commensurate with the exciton forbidden transition at $1503.2 \mathrm{meV}$. Both peaks appear offset from the diagonal, more study is necessary to determine their nature and if they are indeed scattering and bound state or a combination including coupling to the nearby states, which is likely occurring.

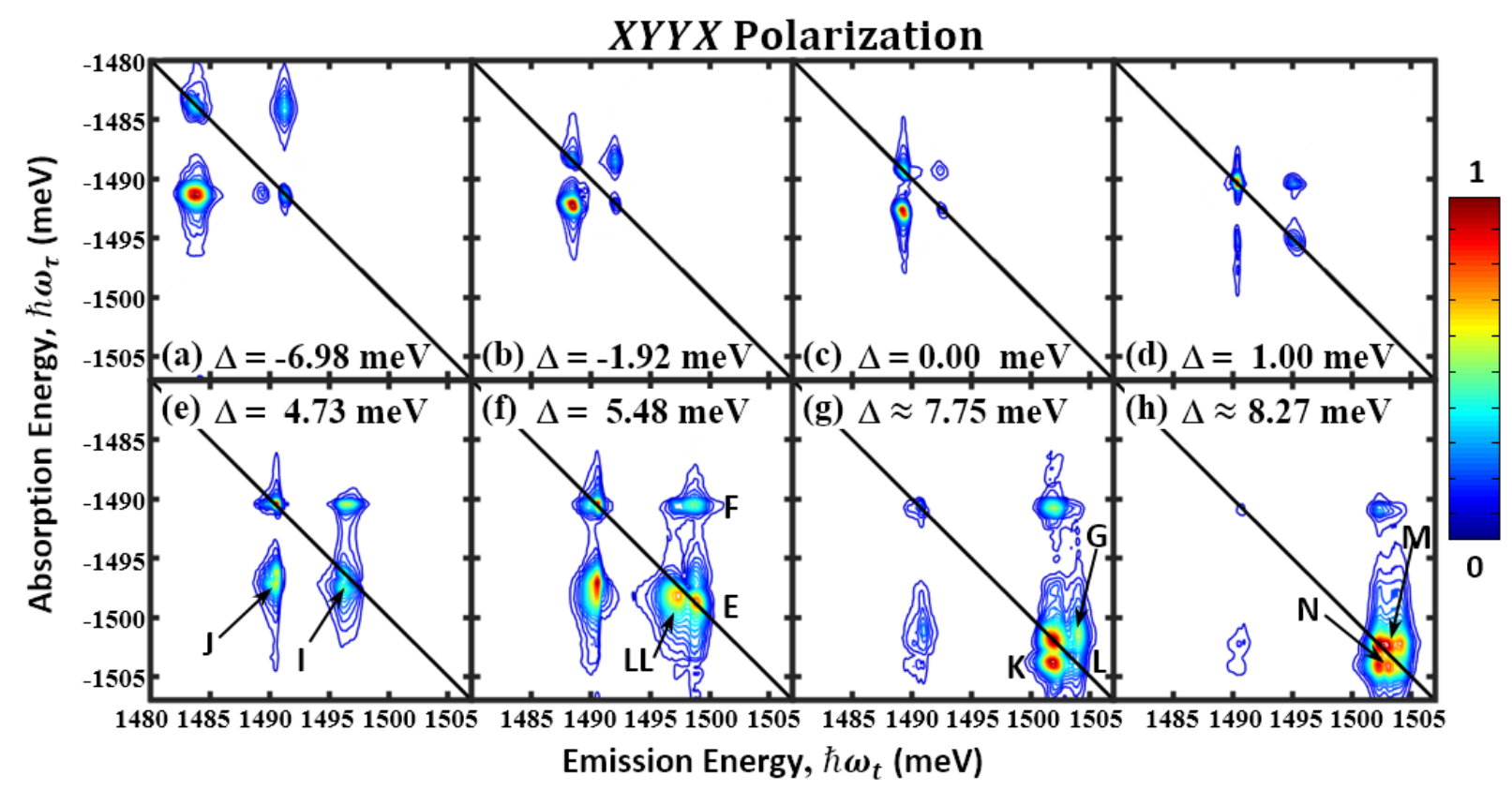

Figure 64 Rephasing $S_{I}$ two-dimensional coherent spectra with crosslinear excitation for the labeled range of detuning. Additional peaks I, J, K, L, N and M appear relative to collinear data.

Under cocircular excitation $\left(\sigma^{+} \sigma^{+} \sigma^{+} \sigma^{+}\right)$, representative spectra shown in Figure 65 (a)(h) are observed. All biexcitonic features are absent (Figure 65 (a)-(c) would otherwise show HH and (f)-(g) for LL), as expected because only one spin species of exciton is present. Features I, J, 
$\mathrm{E}$, and possibly $\mathrm{F}$ emerge, but are considerably weaker than their linear polarization counterparts, see Figure 65 (f), (g). Peak G, the coherence between the 2 s heavy hole exciton and 1 s light hole exciton, also appears in Figure 65 (h) and presumably the peaks $\mathrm{K}$ and $\mathrm{L}$ would appear at more positive detunings.

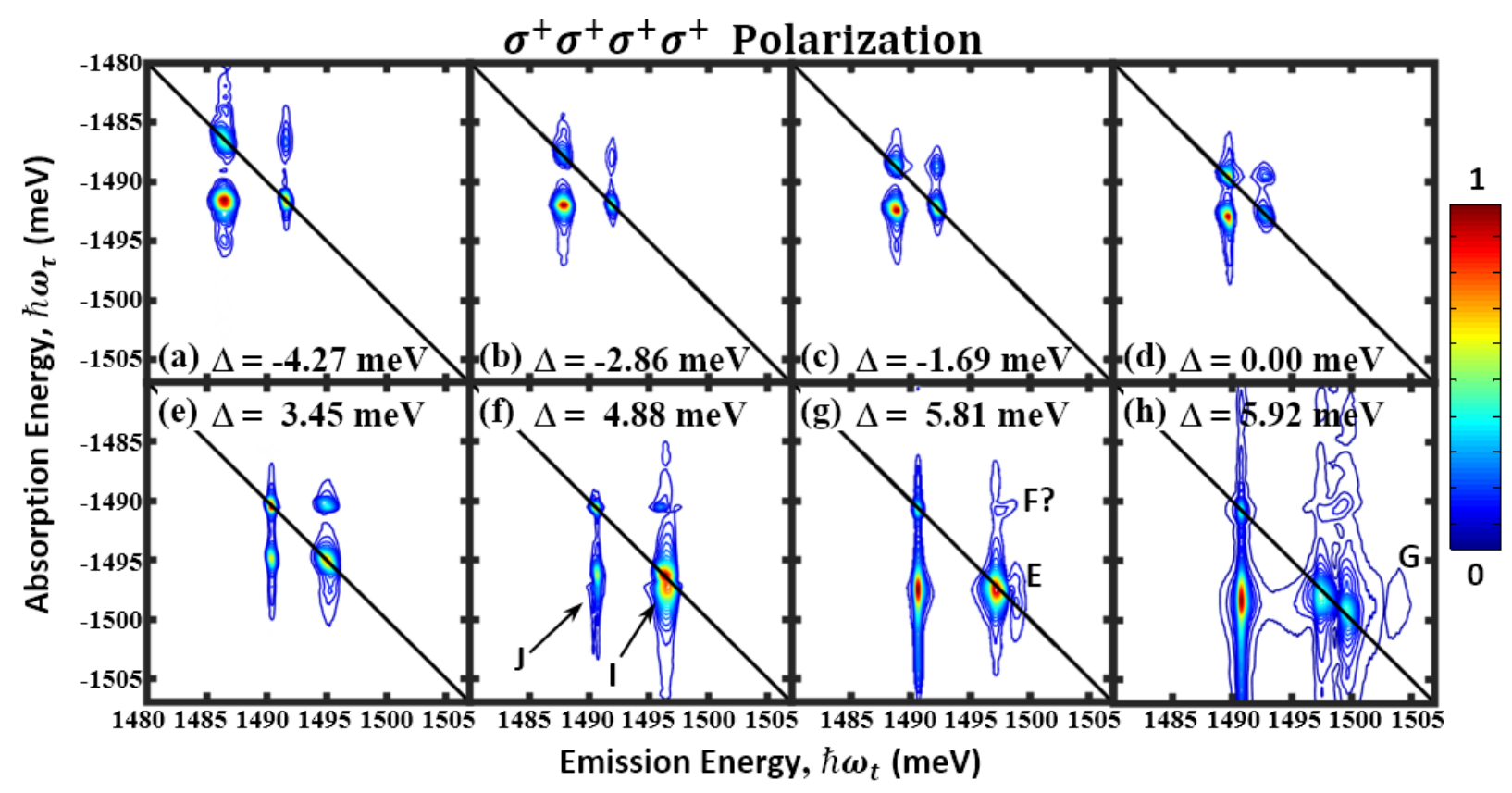

Figure 65 Rephasing $S_{I}$ two-dimensional coherent spectra with cocircular $\left(\sigma^{+} \sigma^{+} \sigma^{+} \sigma^{+}\right)$ excitation for the labeled range of detuning. No additional peaks are observed.

2DCS spectra under crosscircular excitation $\left(\sigma^{-} \sigma^{-} \sigma^{+} \sigma^{+}\right)$are shown in Figure 66. Under this scheme, $\mathrm{MBI}$ are also minimal and biexcitons are encouraged. Not seen in the collinear data, the heavy hole biexciton $\mathrm{HH}$ persists into positive detuning in these one-quantum spectra. In Figure 65 (d), it strongly modifies the spectral peak of the lower polariton branch A, seeming to decrease as they begin to overlap and then A either recovers or is enhanced as the overlap ends, perhaps a result of $\mathrm{HH}$ itself decaying in intensity. This result substantiates the earlier result that the $\mathrm{HH}$ biexciton persists and modifies the systems response at the point where the LP and HH overlap. 


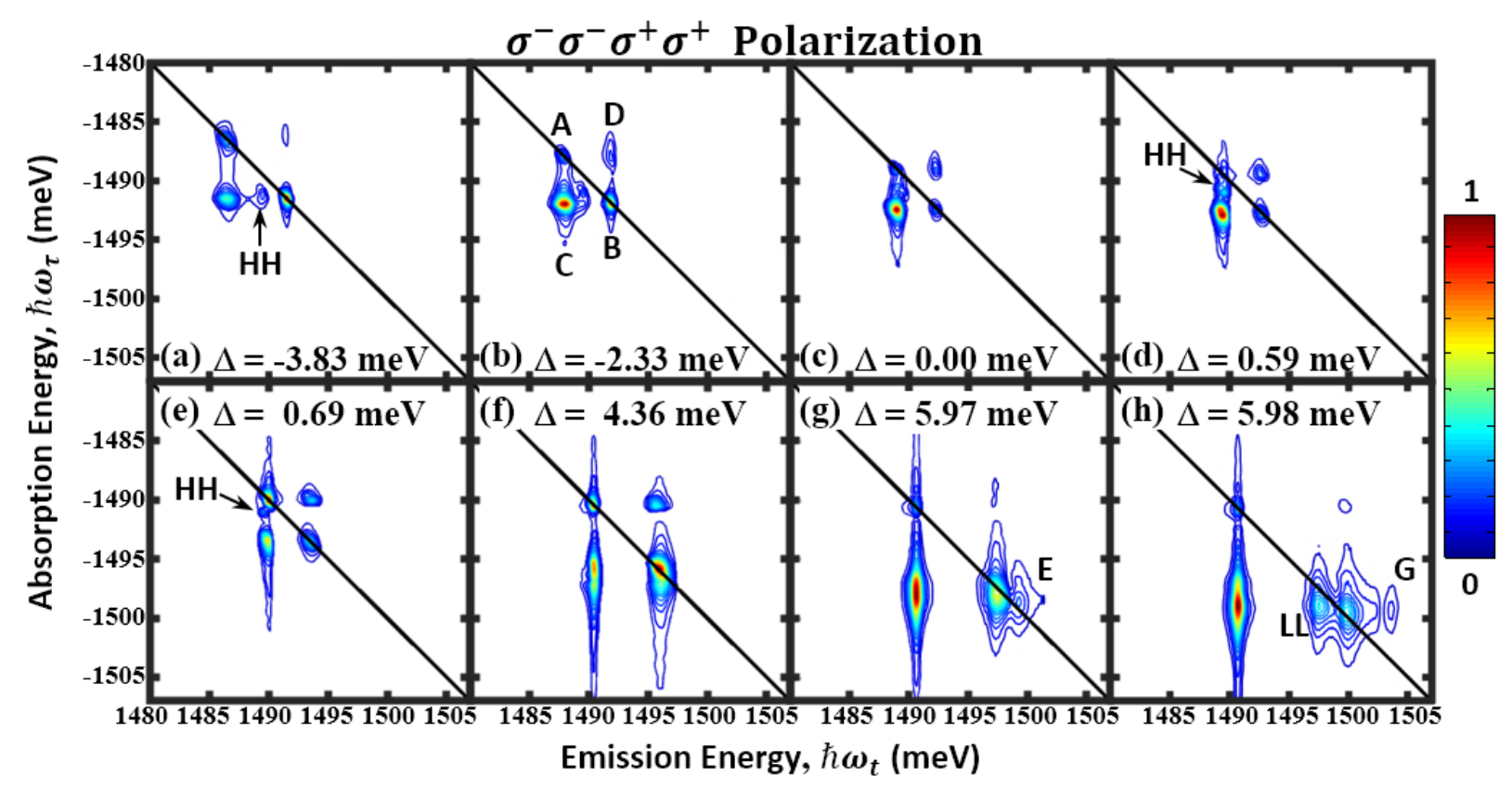

Figure 66 Rephasing $S_{I}$ two-dimensional coherent spectra with crosscircular $\left(\sigma^{-} \sigma^{-} \sigma^{+} \sigma^{+}\right)$ excitation for the labeled range of detuning. No additional peaks are observed

Extracting the integrated peak intensities and linewidths of the upper and lower polariton branches from the complete data set allows for more quantitative comparison of the system's response under the different polarization configurations. Figure 67 shows the total integrated intensity of peaks A-D as a function of detuning under collinear, crosslinear, cocircular, and crosscircular, respectively. Whereas the integrated intensity from Figure 61 (a) results from the normalized SR-FWM, Figure 67 should isolate just the response of the coupled polaritons. However, this is complicated by the new peaks' close proximity to peaks A-D and similarly introduces noise into the linewidths as well. Also, in comparing the total integrated intensities of different scans, absolute intensities must be retrieved to correct for attenuation in the signal path (there in order to not saturate the detector as well as irises to reduce detected scatter). Assuming good filter correction, any change in signal intensity over time not resulting from of the scan itself or ND filters introduces error in the corrected intensity counts. Relative intensity comparisons are of course more immune to these errors. The small error bars shown represent the error associated with scans repeated immediately with no intentional changes to the experiment. Thus the random and systematic errors, at least on the time scale of approximately a few hours, are relatively low. 
With these limitations in mind, one- and two- Gaussian fits are used to coarsely fit the summed A-D integrated data. As expected, for all polarization schemes the system's response peaks near zero detuning where the cavity mode and exciton are energetically aligned. Both linear polarizations Figure 67 (a) and (b) show an overall stronger response compared to circular polarizations Figure 67 (c) and (d). This is a result of the greater number of quantum pathways (or, Feynman diagrams) for linear excitation compared to circular. Collinear excitation, Figure 67 (a), shows an asymmetry about zero with a second possible peak at positive detuning, in the range of 2-6 meV where the new peaks E-H, LL emerge. Unfortunately, the crosslinear data is too noisy to make a definitive conclusion, though it seems to show some increases in intensity at both negative and positive detunings, which could be a result of $\mathrm{MBI}$ being reduced and a stronger biexciton moving into the integration region of peak C. Cocircular Figure 67 (c) shows possibly a flatter response of the system, with two, wide Gaussian profiles of roughly fitting intensity profile.

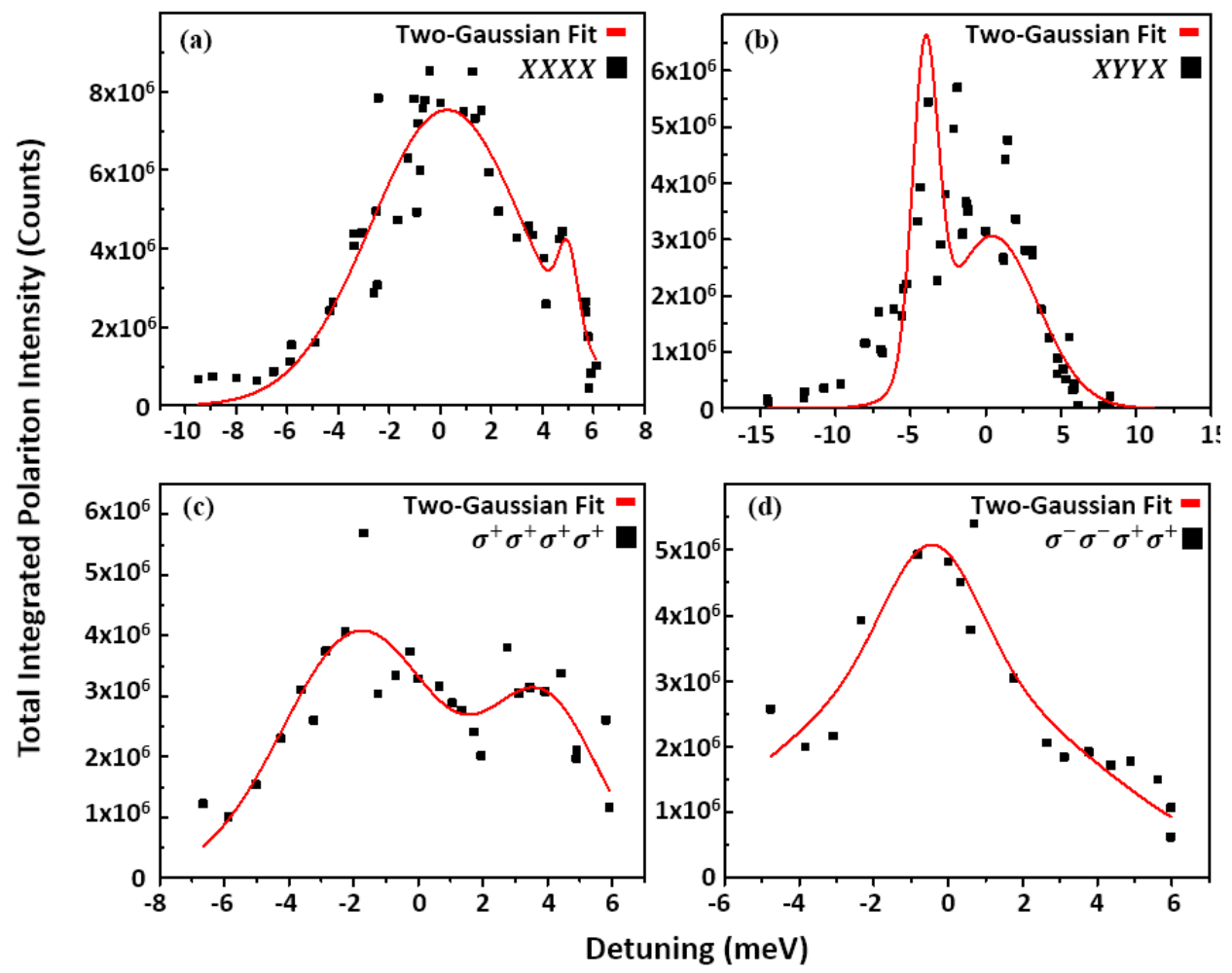

Figure 67 The summed integrated intensity of the polaritons and their coherences (peaks A-D) under (a) collinear, (b) crosslinear, (c) cocircular, and (d) crosscircular polarization excitation. 
This reflects the smaller number of Feynman diagrams and accordingly less complicated spectra, having less peaks than other polarization configurations. Crosscircular polarization Figure 67(d), shows a relatively clear and symmetric peak about zero detuning, suggesting that possibly both spin species of exciton being present generate the strongest response. To further understand the system's response the coupling between the exciton and polariton as function of polarization should be considered.

The relative intensity of peaks A-D are shown in Figure 68 (co- and crosslinear) and Figure 69 (co- and crosscircular). In both collinear and cociruclar polarizations, from negative to positive detuning, the upper polariton starts stronger than the lower, and become equal amplitude near $\Delta \sim-2 \mathrm{meV}$ and then diverge again, peaking at $\Delta \sim 0$, with the upper branch again stronger than the lower. At $\Delta \sim+2 \mathrm{meV}$ the diagonal peaks come together and swap relative intensity, with the lower branch now relatively stronger, see Figure 68 (a) and Figure 69 (a). Their off-diagonal coherences (collinear in Figure 68(b) and cocircular in Figure 69(b)) follow a somewhat similar trend. Coherence $\mathrm{C}$ is stronger than $\mathrm{D}$ except near $\Delta \geq \sim 2 \mathrm{meV}$ where they come together and possibly flip. C and D also begin to come together slightly near-(2-4) meV. 


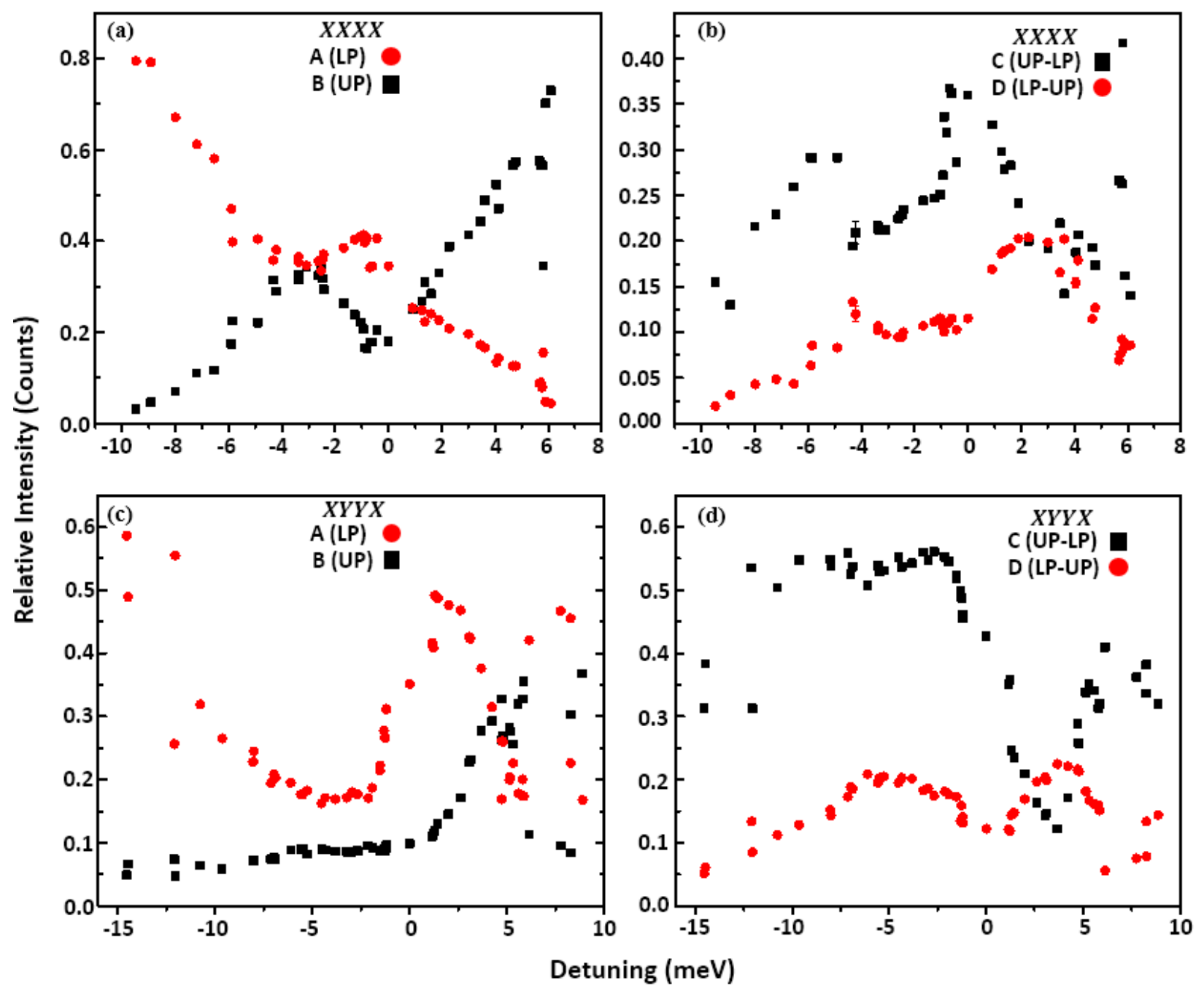

Figure 68 The relative integrated intensity of the (a), (c) diagonal coherences and (b), (d) offdiagonal coherences under (a), (b) collinear and (c), (d) crosslinear excitation.

The relative integrated intensities of the diagonal peaks under crosslinear and crosscircular polarization show different trends than their collinear and crosslinear counterparts, see Figure 68(c) and Figure 69(c). Under crosslinear excitation, they do not come together (and possibly invert) except at larger, $\sim 5 \mathrm{meV}$, detuning. Though they do trend towards each other at $\Delta \sim-5-0 \mathrm{meV}$. After zero detuning, there is a marked increase of both peaks. Under cocircular excitation at negative detuning, both diagonal coherences are of comparable intensity, separate near zero, and come together again near $\Delta \sim 2$ and $5 \mathrm{meV}$. This is likely an effect of the heavy hole biexciton being switched off for these negative detunings. The coherences' relative 
intensities of both polarizations have the same general behavior of coming together and again separating near $3 \mathrm{meV}$ detuning.

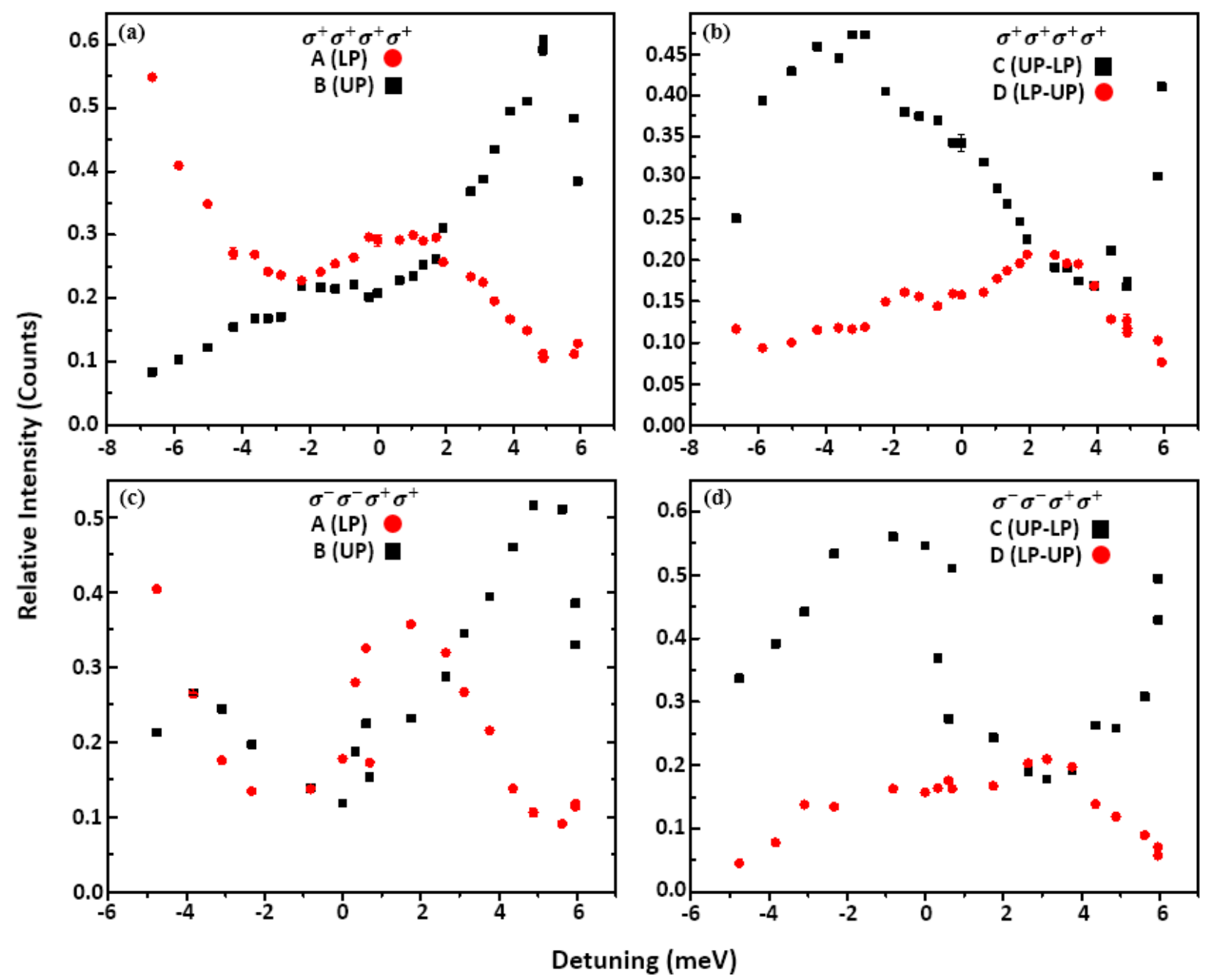

Figure 69 The relative integrated intensity of the (a), (c) diagonal coherences and (b), (d) offdiagonal coherences under (a), (b) cocircular and (c), (d) crosscircular excitation. 

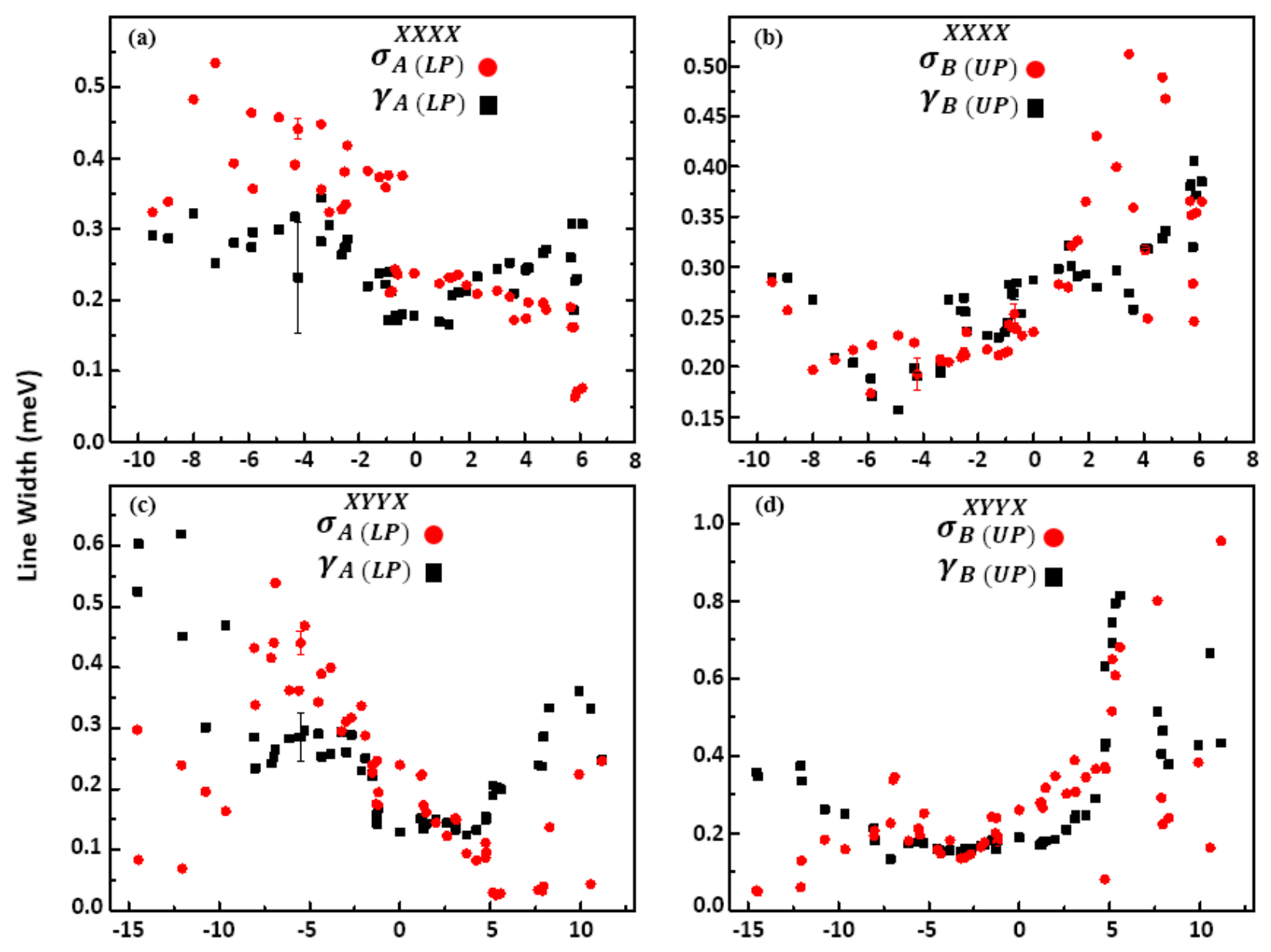

Detuning (meV)

Figure 70 Homogeneous and inhomogeneous widths of peaks A and B under collinear and crosslinear polarization excitation.

The homogeneous and inhomogeneous linewidths of peaks $\mathrm{A}$ and $\mathrm{B}$ are denoted $\gamma_{A}, \sigma_{A}$ and $\gamma_{B}, \sigma_{B}$, respectively, and shown in Figure 70 and Figure 71 under the four polarization schemes. In all cases, one can see an (albeit noisy) trend that peaks A and B show reciprocal behavior. This behavior reflects the Hopfield coefficients of the upper and lower polariton branches (the two swap which is exciton or cavity-like as a function of detuning). For example, under cocircular excitation Figure 71(a), (b) the widths from peak A decrease while those form B increase and assume roughly the same values. Also, the amount of homogeneous broadening and inhomogeneous broadening swap about zero for each peak, with peak A having more inhomogeneity than homogeneity (and vice versa for B) at negative detuning in most cases besides circular where the homogeneous and inhomogeneous widths of B may be comparable. 
This implies that a chief reference of inhomogeneity in the sample is the wedge of the cavity of itself, as $A$ is the cavity-like polariton at negative detunings (and $B$ is exciton-like). Also the outlier in Figure $71(\mathrm{c})$ at $\sim 1 \mathrm{meV}$ detuning occurs where the biexciton overlaps and seems to destructively interfere with peak $A$.

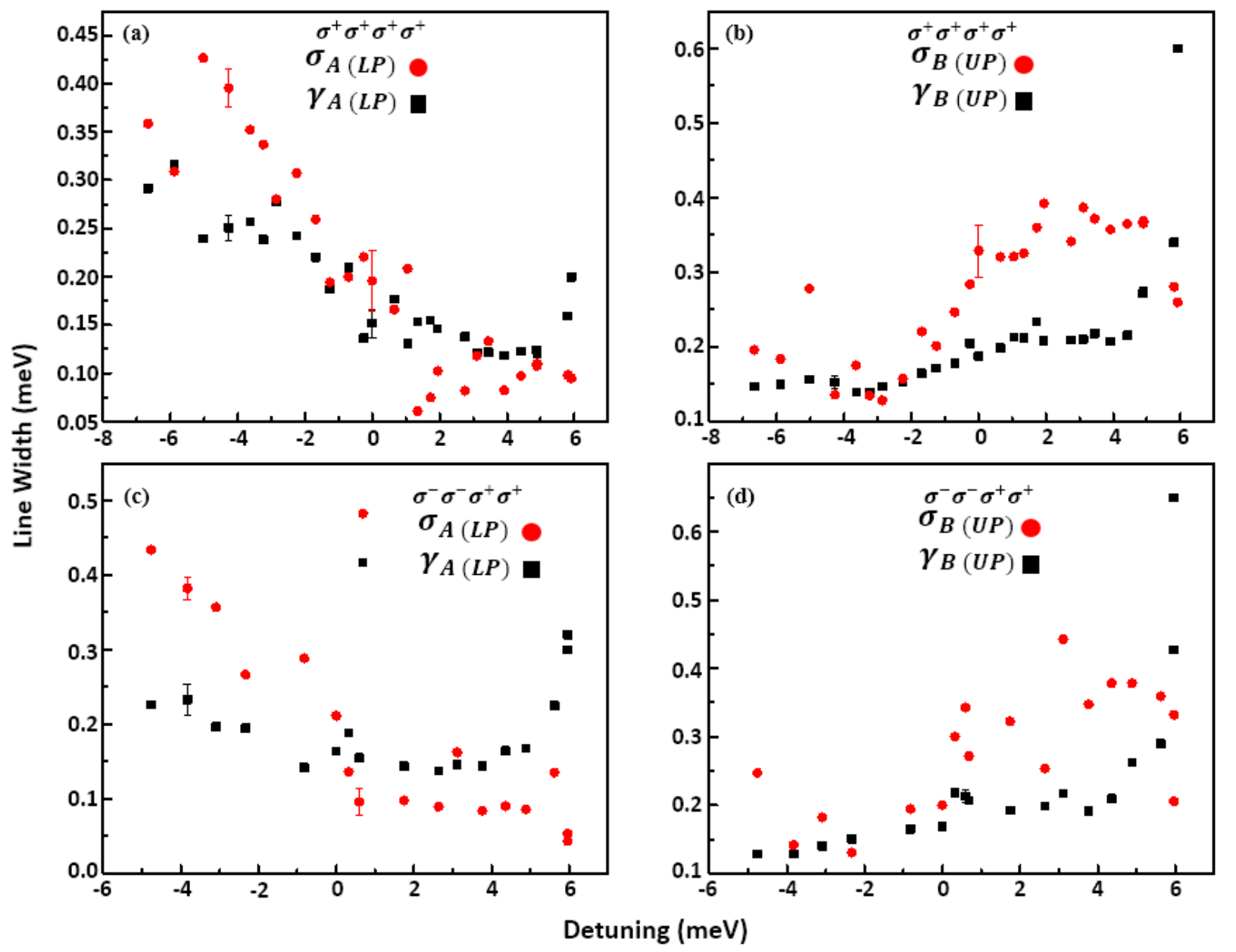

Figure 71 Homogeneous and inhomogeneous widths of peaks A and B under collinear and crosslinear polarization excitation

The error associated with the polariton peaks weakening and decaying to nothing and new states emerging and strengthening at extreme detunings makes interpretation of the data in those regions difficult.

One last possible observation is that circular compared to linear, and furthermore cocircular compared to crosscircular, shows less noisy behavior. Assuming at least some of this behavior is due to actual fine oscillations of the peak widths, it is a measure of the number of 
pathways contributing to the spectrum in this state-rich system. Cociruclar data, having the least number of pathways (particularly no biexcitonic contributions), shows less complicated behavior as a result of less states being able to interact, reflecting the high sensitivity of the linewidths to microscopic many body interactions.

\subsection{Two-Quantum Data}

These $2 \mathrm{Q}, S_{I I I}$ results are acquired under collinear polarization, where numerous excitonpolariton and biexciton-polariton pathways are allowed by optical dipole selection rules, hence suggesting that the off diagonal features are highly sensitive to interactions between $1 Q$ and $2 Q$ pathways. The interaction between these pathways is analogous to atomic and molecular transitions coming into resonance in cold atomic systems, which is known as a Feshbach resonance. $[103,195,196]$ Measuring additional quantum pathways will facilitate better understanding of the differences between purely bosonic and these mostly bosonic systems. Non-rephasing $2 \mathrm{Q}$ spectra allow for disambiguation of the role doubly excited states, such as the biexciton and two-polariton states, play in the third-third order polarization. Figure 72 shows $2 Q$ spectra, at very similar negative detunings recorded with collinear Figure 72(a) and co-circular Figure 72 (b) polarization excitation [197]. For collinear excitation, the optical dipole selection rules allow biexcitons and spin pairing of polaritons. As previously seen by Wen et al, these results exhibit pathways from two correlated lower and upper polaritons ( $\mathrm{LP}_{2}$ and $\mathrm{UP}_{2}$ respectively) and two mixed states consisting of one upper and one lower correlated polaritons, called mixed polaritons $\left(\mathrm{MP}_{2}\right)$. [52] Two mixed peaks exists because each $\mathrm{MP}_{2}$ can interfere with the radiation from the $1 Q$ coherences (LP and UP). LP emission is preferred due to many body interactions [180]. Interestingly for the different polarizations, the $\mathrm{MP}_{2}$ have more relative spectral wright when biexcitons are suppressed, indicating that the presence of biexcitons reduces or distinctly perturbs the polaritonic coupling. 

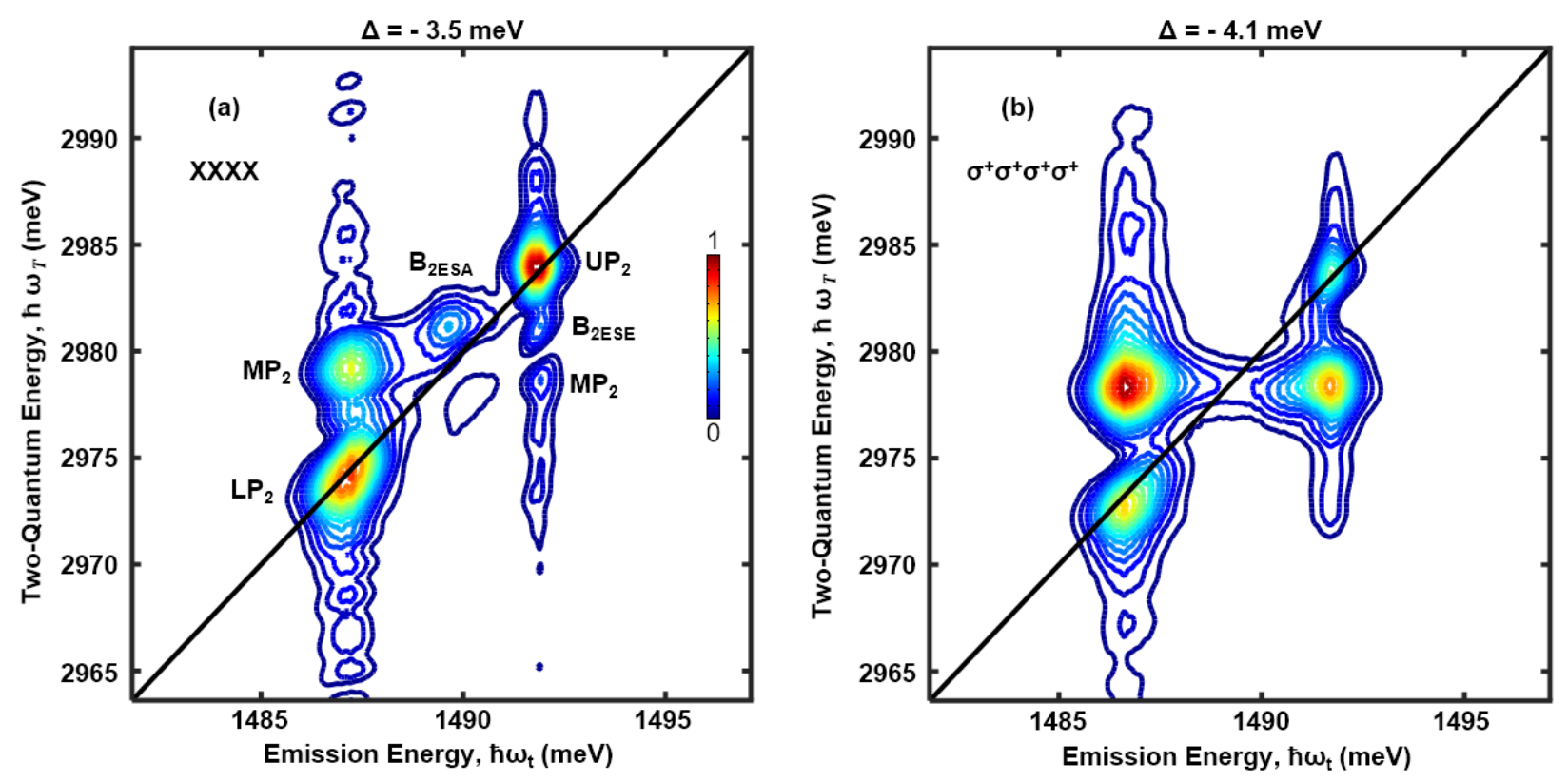

Figure 72 Two-quantum, $S_{I I I}$ spectra at very similar negative detunings with collinearly (XXXX) and co-circularly $\left(\sigma^{+} \sigma^{+} \sigma^{+} \sigma^{+}\right)$polarized respectively with low irradiance $(4.23 \mathrm{~mW} / \mathrm{cm} 2)$ excitation. The $2 \mathrm{Q}$ features for the lower, mixed, and upper polaritons are denoted $L P_{2}, \mathrm{MP}_{2}$ and $\mathrm{UP}_{2}$. Since $U_{2}$ is mostly exciton-like it can couple to bound heavy hole biexcitons, denoted $B$. There are two biexcitonic peaks $B_{2 E S A}$ and $B_{2 E S E}$ referring to the Feynman diagrams in Figure 42 . Reproduced from reference [197]. 
For collinear polarized excitation, additional peaks are observed which were not reported by Wen et al [52]. Features $B_{2 E S E}$ and $B_{2 E S A}$ are seen directly below the UP 2 peak, offset by the biexciton binding energy $(\sim-2 \mathrm{meV})$. The former is directly under $\mathrm{UP}_{2}$ and corresponds to a $2 \mathrm{Q}$ excited-state emission pathway, meaning that the emission ends in the ground state. The latter is offset along the emission axis as well by the binding energy and corresponds to a $2 \mathrm{Q}$ excitedstate absorption pathway, meaning that the emission ends in the UP excited state. [198] Wen et al have a stronger cavity-photon coupling than in this work, exhibiting a vacuum Rabi splitting that is nearly double the $4 \mathrm{meV}$ presented here. Additionally, their work reports spectra at $\Delta=$ $1 \mathrm{meV}$. As seen in $1 \mathrm{Q}$ spectra presented in Figure 60, the biexciton disappears as the detuning become small and the biexciton overlaps the LP. Due to the conditions in the previous work, it is therefore unlikely they would have observed clearly isolated biexciton features.

\subsection{Zero-Quantum Data}

Figure 73 shows the rephasing $0 Q, S_{I}$ spectra, acquired at negative $(-4.8 \mathrm{meV})$ and nearzero (-0.3 meV) detuning with collinear excitation and low irradiance. Continuing the concept of $2 \mathrm{Q}$ and $1 \mathrm{Q}$ spectra, the $\mathrm{OQ}$ spectra show transitions that do not directly emit into the signal, but are a result of Raman-like coherences during the mixing time $(T)$, due to coherences between excited state populations. Both $0 \mathrm{Q}$ spectra in Figure 73 reveal the features RC1 and RC2 in the more negatively detuned spectra. Asymmetry in the strengths of the two features is observed and can be expected within a model that includes many-body interactions [199] and favoring the lower energy Raman-like coherence. Approaching zero detuning enhances all the spectral features and increases the asymmetry between the Raman-like coherences. The enhancement is commensurate with those observed in all other features as the light-matter interactions increase near $\Delta=0$. Noted that vertical position of Raman-like coherences is not directly comparable between the spectra, because they are defined by the separation of the LP and UP, which varies with detuning. Feature RC1 increases from approximately $10 \%$ to $35 \%$ of the LP features when approaching zero detuning, while RC2 nearly completely vanishes. This result indicates that for this polarization many-body interactions increase as the light-matter interactions increase. 

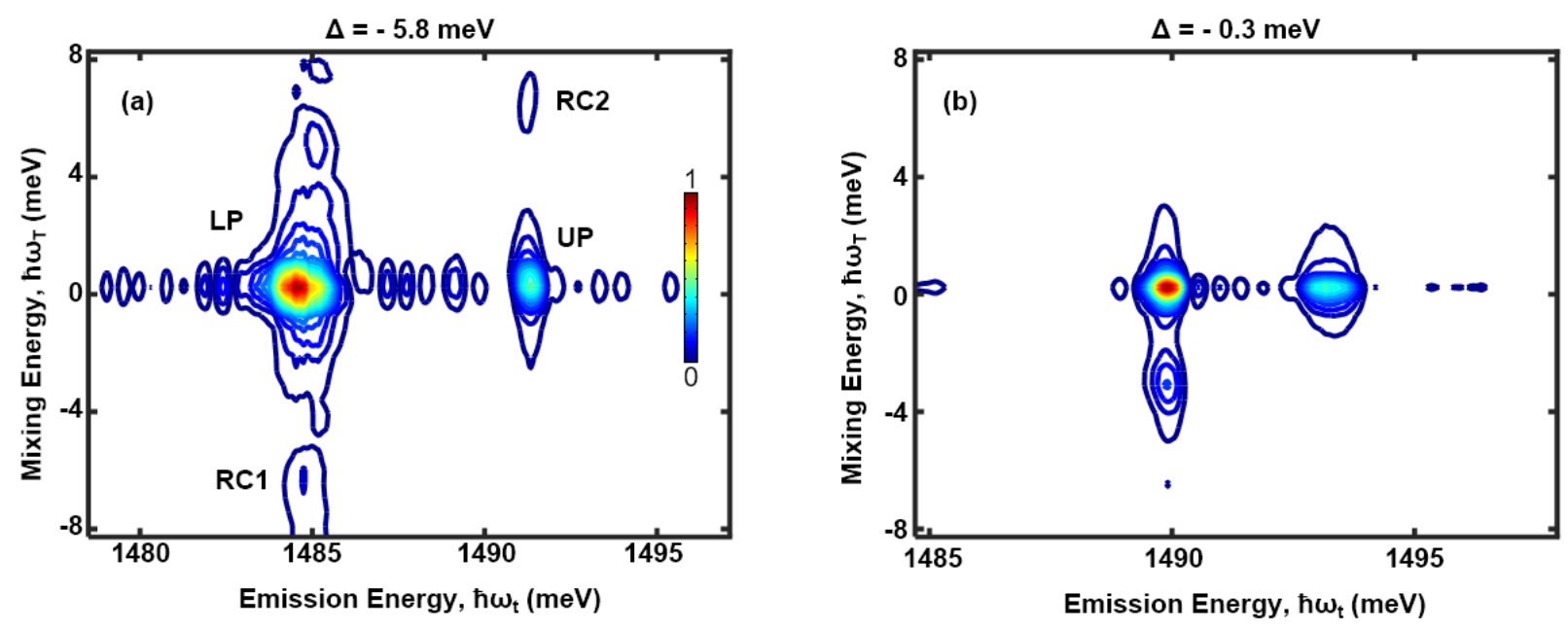

Figure 73 Zero-quantum spectra negatively detuned at (a) $-5.8 \mathrm{meV}$ and near zero at (b) -0.3 meV. Both spectra are under collinear $(X X X X)$ and low irradiance $(4.23 \mathrm{~mW} / \mathrm{cm} 2)$ excitation. Reproduced from reference [197].

\subsection{Conclusions from Study of a Quantum Microcavity}

In summary, this study has mapped the detuning dependence of the cavity mode through the exciton and biexciton modes of a single quantum well and isolated the coherent response two-dimensional coherent spectroscopy, examining the zero-, one-, and two-quantum energy manifolds. Enhancement of the four-wave mixing emission was observed near zero detuning, along with anticrossing of the upper and lower polariton branches as well as a biexciton contribution which becomes a Feshbach resonance as the biexciton and lower polariton interfere. Homogeneous and inhomogeneous linewidths are consistent with those for a wider cavity mode than the bare exciton mode. Polarization dependence of both the linewidths and the amplitudes of the spectral features are highly sensitive to $\mathrm{MBI}$ and the interaction between exciton, biexciton, and cavity modes, revealing strong modification as bands intersect.

Second, the polarization dependence of $2 \mathrm{Q}$ spectra has been shown to control the biexciton contributions, revealing two new quantum pathways for negative detuning and a perturbation in the polaritonic coupling due to the biexciton. Finally, $\mathrm{OQ}$ spectra for two different detunings, show Raman-like coherences that increase in strength and asymmetry close to zero- 
detuning and indicating that many-body (matter-matter) interactions increase along with the light-matter interactions. This work paves the way for deeper understanding of biexciton interactions and their effect on polaritonic coupling in semiconductor microcavities.

This work paves the way for determining contributions through polarization- and excitation-dependent studies using 2DCS and begs full microscopic theoretical treatment to reproduce spectral features. Moreover, these methods can be used to disentangle the coherent and transient phenomena that parallel processes identified in ultracold atomic physics, such as condensation and superfluidity 


\section{Chapter 6 Conclusion}

In this work, MDCS has yielded information regarding fundamental excitations of model systems, which is applicable not just to the GaAs and InGaAs structures considered here but also yields insight into all direct-bandgap semiconductor systems.

In bulk GaAs, we have observed that even small amounts of strain change the nature of the excitons. With increasing strain and degeneracy breaking between the heavy- and light-hole valence bands, the resulting heavy- and light- hole exciton become increasingly dissimilar, as evidenced by their changing homogeneous and inhomogeneous linewidths. With sufficient strain, biexcitonic states can form in the bulk sample which have not previously been observed. Their biexcitonic nature is confirmed with polarization dependent measurements which enhances their peaks as well as two-quantum measurements which show several bound and unbound two-exciton complexes. To more clearly understand the effect of strain on the exciton and biexcitonic contributions, two-quantum measurements ought to be conducted on both the high- and low-strain sample. Though, ideally, all measurements should be conducted with a continuously variable amount of strain, starting with zero strain and degenerate heavy- and lighthole states.

The normal-mode coupling in a quantum microcavity between the photonic cavity mode and the exciton resonance enhances both the linear and nonlinear optical interactions [85] and despite the extensive studies on this system, it has not been systematically mapped using MDCS. This study has mapped the detuning dependence of the cavity mode through the exciton and biexciton modes of a single InGaAs quantum well. Enhancement of the four-wave mixing signal was observed near zero detuning as well as a biexciton contribution which becomes a Feshbach resonance as the biexciton and lower polariton interfere. Varying excitation polarization shows the linewidths and the amplitudes of the spectral features are highly sensitive to many-body interactions as well as the interaction between the polaritonic and excitonic transitions. At large positive detunings, possible dark excitons emerge and couple to the polaritonic states, which have not been previously observed. Two-quantum spectra show biexcitonic and bipolaritonic contributions. Zero-quantum spectra show Raman-like coherences that increase in strength and 
asymmetry close to zero-detuning and indicating that many-body (matter-matter) interactions increase along with the light-matter interactions. To elucidate the role two-quantum states and play in the nonlinear response, two- and one-quantum measurements a function of polarization should be conducted as well as excitation density dependent and temperature dependent measurements to study bound states under the influence of increase dephasing mechanisms. This work paves the way for determining contributions through polarization- and excitationdependent studies using 2DCS and begs full microscopic theoretical treatment to reproduce spectral features. Moreover, these methods can be used to disentangle the coherent and transient phenomena that parallel processes identified in ultracold atomic physics, such as condensation and superfluidity. 


\section{Publications}

D. Webber, B.L. Wilmer, X. Liu, M. Dobrowolska, J.K. Furdyna, A.D. Bristow, and K.C. Hall, Phys. Rev. B 94, 155450 (2016)

B.L. Wilmer, D. Webber, J.M. Ashley, K.C. Hall, and A.D. Bristow, Phys. Rev. B 94, 075207 (2016).

B. L. Wilmer, Proc. Soc. Photogr. Instrumentation Eng. 9746, (2016).

B.L. Wilmer, F. Passmann, M. Gehl, G. Khitrova, and A.D. Bristow, Phys. Rev. B Rapid Comm. 91, 201304(R) (2015).

\section{Bibliography}

[1] J. Li, S. K. Cushing, F. Meng, T. R. Senty, A. D. Bristow, and N. Wu, Nat. Photonics 9, 601 (2015).

[2] M. A. Green, K. Emery, Y. Hishikawa, W. Warta, and E. D. Dunlop, Prog. Photovolt. Res. Appl. 23, 1 (2015).

[3] A. Imamoglu, R. J. Ram, S. Pau, Y. Yamamoto, and others, Phys. Rev. A 53, 4250 (1996).

[4] Daniele Bajoni, P. Senellart, E. Wertz, I. Sagnes, A. Miard, A. Lemaitre, and J. Bloch, Phys. Rev. Lett. 100, 47401 (2008).

[5] Shuji Nakamura, Takashi Mukai, and Masayuki Senoh, Jpn. J. Appl. Phys. 30, 1998 (1991).

[6] T. Mueller, F. Xia, and P. Avouris, Nat. Photonics 4, 297 (2010).

[7] J. Lee, P. Jadhav, and M. A. Baldo, Appl. Phys. Lett. 95, 33301 (2009).

[8] S. Manna, S. Das, S. P. Mondal, R. Singha, and S. K. Ray, J. Phys. Chem. C 116, 7126 (2012).

[9] J. Michel, J. Liu, and L. C. Kimerling, Nat. Photonics 4, 527 (2010).

[10] R. H. Hadfield, Nat. Photonics 3, 696 (2009).

[11] A. Argyris, D. Syvridis, L. Larger, V. Annovazzi-Lodi, P. Colet, I. Fischer, J. GarcíaOjalvo, C. R. Mirasso, L. Pesquera, and K. A. Shore, Nature 438, 343 (2005).

[12] N. Schlosser, G. Reymond, I. Protsenko, and P. Grangier, Nature 411, 1024 (2001).

[13] J. Armstrong, J. Light. Technol. 27, 189 (2009).

[14] J. S. Foresi, P. R. Villeneuve, J. Ferrera, E. R. Thoen, G. Steinmeyer, S. Fan, J. D. Joannopoulos, L. C. Kimerling, H. I. Smith, and E. P. Ippen, Nature 390, 143 (1997).

[15] C. H. Bennett and D. P. DiVincenzo, Nature 404, 247 (2000).

[16] D. P. DiVincenzo, Quantum Comput. Quantum Inf. Theory Repr. Vol. Introd. Notes ISI TMR Netw. Sch. 12-23 July 1999 Villa Gualino Torino Italy 131 (2000).

[17] Proc. R. Soc. Lond. A 439, 553 (1992).

[18] H. Li, A. D. Bristow, M. E. Siemens, G. Moody, and S. T. Cundiff, Nat. Commun. 4, 1390 (2013).

[19] D. B. Turner, K. W. Stone, K. Gundogdu, and K. A. Nelson, J. Chem. Phys. 131, 144510 (2009).

[20] Z. Zhang, P. H. Lambrev, K. L. Wells, G. Garab, and H.-S. Tan, Nat. Commun. 6, 7914 (2015). 
[21] X. Li, T. Zhang, C. N. Borca, and S. T. Cundiff, Phys. Rev. Lett. 96, 57406 (2006).

[22] S. T. Cundiff, T. Zhang, A. D. Bristow, D. Karaiskaj, and X. Dai, Acc. Chem. Res. 42, 1423 (2009).

[23] Wolfgang Langbein and Brian Patton, Opt. Lett. 31, 1151 (2006).

[24] D. B. Turner and K. A. Nelson, Nature 466, 1089 (2010).

[25] J. Kasprzak, B. Patton, V. Savona, and W. Langbein, Nat. Photonics 5, 57 (2011).

[26] C. R. Hall, J. O. Tollerud, H. M. Quiney, and J. A. Davis, New J. Phys. 15, 45028 (2013).

[27] P. Dey, J. Paul, J. Bylsma, S. Deminico, and D. Karaiskaj, Rev. Sci. Instrum. 84, 23107 (2013).

[28] L. Yang, T. Zhang, A. D. Bristow, S. T. Cundiff, and S. Mukamel, J. Chem. Phys. 129, 234711 (2008).

[29] A. D. Bristow, T. Zhang, M. E. Siemens, S. T. Cundiff, and R. P. Mirin, J. Phys. Chem. B 115, 5365 (2011).

[30] W. P. Aue, E. Bartholdi, and R. R. Ernst, J. Chem. Phys. 64, 2229 (1976).

[31] R. R. Ernst, G. Bodenhausen, and A. Wokaun, Principles of Nuclear Magnetic Resonance in One and Two Dimensions (Oxford Science Publications, Oxford, 1987).

[32] John D. Hybl, Allison W. Albrecht, Sarah M. Gallagher Faeder, and David M. Jonas, Chem. Phys. Lett. 297, 307 (1998).

[33] J. Hybl, A. Ferro, and D. Jonas, J. Chem. Phys. 115, 6606 (2001).

[34] M. C. Asplund, M. T. Zanni, and R. M. Hochstrasser, Proc. Natl. Acad. Sci. 97, 8219 (2000).

[35] O. Golonzka, M. Khalil, N. Demirdoven, and A. Tokmakoff, J. Chem. Phys. 115, 10814 (2001).

[36] M. Woerner, W. Kuehn, P. Bowlan, K. Reimann, and T. Elsaesser, New J. Phys. 15, 25039 (2013).

[37] C. Tseng, S. Matsika, and T. C. Weinacht, Opt. Express 17, 18788 (2009).

[38] B. A. West, B. P. Molesky, P. G. Giokas, and A. M. Moran, Chem. Phys. 423, 92 (2013).

[39] R. D. Mehlenbacher, T. J. McDonough, M. Grechko, M.-Y. Wu, M. S. Arnold, and M. T. Zanni, Nat. Commun. 6, 6732 (2015).

[40] G. Moody, M. E. Siemens, A. D. Bristow, X. Dai, A. S. Bracker, D. Gammon, and S. T. Cundiff, Phys. Rev. B 83, 245316 (2011).

[41] G. Moody, R. Singh, H. Li, I. A. Akimov, M. Bayer, D. Reuter, A. D. Wieck, A. S. Bracker, D. Gammon, and S. T. Cundiff, Phys. Rev. B 87, (2013).

[42] T. Zhang, I. Kuznetsova, T. Meier, X. Li, R. P. Mirin, P. Thomas, and S. T. Cundiff, Proc. Natl. Acad. Sci. 104, 14227 (2007).

[43] B. L. Wilmer, F. Passmann, M. Gehl, G. Khitrova, and A. D. Bristow, Phys. Rev. B 91, 201304(R) (2015).

[44] V. M. Huxter, T. A. A. Oliver, D. Budker, and G. R. Fleming, Nat. Phys. 9, 744 (2013).

[45] D. D. Kohler, S. B. Block, S. Kain, A. V. Pakoulev, and J. C. Wright, J. Phys. Chem. C 118, 5020 (2014).

[46] Y. Kobayashi, C.-H. Chuang, C. Burda, and G. D. Scholes, J. Phys. Chem. C 118, 16255 (2014).

[47] X. Dai, A. D. Bristow, D. Karaiskaj, and S. T. Cundiff, Phys. Rev. A 82, 52503 (2010).

[48] M. Ferretti, R. Hendrikx, E. Romero, J. Southall, R. J. Cogdell, V. I. Novoderezhkin, G. D. Scholes, and R. van Grondelle, Sci. Rep. 6, 20834 (2016).

[49] L. Yang, I. V. Schweigert, S. T. Cundiff, and S. Mukamel, Phys. Rev. B 75, 125302 (2007). 
[50] D. Karaiskaj, A. D. Bristow, L. Yang, X. Dai, R. P. Mirin, S. Mukamel, and S. T. Cundiff, Phys. Rev. Lett. 104, 117401 (2010).

[51] K. W. Stone, K. Gundogdu, D. B. Turner, X. Li, S. T. Cundiff, and K. A. Nelson, Science 324, 1169 (2009).

[52] P. Wen, G. Christmann, J. J. Baumberg, and K. A. Nelson, New J. Phys. 15, 25005 (2013).

[53] A. D. Bristow, D. Karaiskaj, X. Dai, T. Zhang, C. Carlsson, K. R. Hagen, R. Jimenez, and S. T. Cundiff, Rev Sci Instrum 80, 73108 (2009).

[54] A. D. Bristow, D. Karaiskaj, X. Dai, and S. T. Cundiff, Opt. Express 16, 18017 (2008).

[55] C. H. Gooch, C. Hilsum, and B. R. Holeman, J. Appl. Phys. 32, 2069 (1961).

[56] M. V. Hobden and M. D. Sturge, Proc. Phys. Soc. 78, 615 (1961).

[57] M. D. Sturge, Phys. Rev. 127, 768 (1962).

[58] H. Wang, K. Ferrio, D. G. Steel, Y. Z. Hu, R. Binder, and S. W. Koch, Phys. Rev. Lett. 71, 1261 (1993).

[59] H. Wang, K. B. Ferrio, D. G. Steel, P. R. Berman, Y. Z. Hu, R. Binder, and S. W. Koch, Phys Rev A 49, R1551 (1994).

[60] I. Kuznetsova, T. Meier, S. T. Cundiff, and P. Thomas, Phys Rev B 76, 153301 (2007).

[61] I. Kuznetsova, N. Gőgh, J. Förstner, T. Meier, S. T. Cundiff, I. Varga, and P. Thomas, Phys. Rev. B 81, 75307 (2010).

[62] M. E. Siemens, G. Moody, H. Li, A. D. Bristow, and S. T. Cundiff, Opt. Express 18, 17699 (2010).

[63] G. Moody, M. E. Siemens, A. D. Bristow, X. Dai, D. Karaiskaj, A. S. Bracker, D. Gammon, and S. T. Cundiff, Phys. Rev. B 83, 115324 (2011).

[64] Y. D. Glinka, Z. Sun, M. Erementchouk, M. N. Leuenberger, A. D. Bristow, S. T. Cundiff, A. S. Bracker, and X. Li, Phys. Rev. B 88, 75316 (2013).

[65] T. Zhang, I. Kuznetsova, T. Meier, X. Li, R. P. Mirin, P. Thomas, and S. T. Cundiff, Proc. Natl. Acad. Sci. 104, 14227 (2007).

[66] A. D. Bristow, D. Karaiskaj, X. Dai, R. P. Mirin, and S. T. Cundiff, Phys. Rev. B 79, 161305(R) (2009).

[67] L. Yang and S. Mukamel, Phys. Rev. Lett. 100, 57402 (2008).

[68] G. Nardin, G. Moody, R. Singh, T. M. Autry, H. Li, F. Morier-Genoud, and S. T. Cundiff, Phys. Rev. Lett. 112, 46402 (2014).

[69] D. Webber, B. L. Wilmer, X. Liu, M. Dobrowolska, J. K. Furdyna, A. D. Bristow, and K. C. Hall, ArXiv160506475 Cond-Mat (2016).

[70] B. Zhao, T. R. Chen, L. E. Eng, Y. H. Zhuang, A. Shakouri, and A. Yariv, Appl. Phys. Lett. 65, 1805 (1994).

[71] D. Leonard, M. Krishnamurthy, C. M. Reaves, S. P. Denbaars, and P. M. Petroff, Appl. Phys. Lett. 63, 3203 (1993).

[72] A.-L. Barabási, Appl. Phys. Lett. 70, 2565 (1997).

[73] D. L. Huffaker, G. Park, Z. Zou, O. B. Shchekin, and D. G. Deppe, IEEE J. Sel. Top. Quantum Electron. 6, 452 (2000).

[74] T. E. Whall and E. H. C. Parker, J. Phys. Appl. Phys. 31, 1397 (1998).

[75] F. Ding, R. Singh, J. D. Plumhof, T. Zander, V. Křápek, Y. H. Chen, M. Benyoucef, V. Zwiller, K. Dörr, G. Bester, A. Rastelli, and O. G. Schmidt, Phys. Rev. Lett. 104, 67405 (2010).

[76] V. Sih, H. Knotz, J. Stephens, V. R. Horowitz, A. C. Gossard, and D. D. Awschalom, Phys. Rev. B 73, 241316 (2006). 
[77] H. Knotz, A. W. Holleitner, J. Stephens, R. C. Myers, and D. D. Awschalom, Appl. Phys. Lett. 88, 241918 (2006).

[78] Y. Zhang, B. J. Skromme, and F. S. Turco-Sandroff, Phys. Rev. B 46, 3872 (1992).

[79] K. J. Moore, G. Duggan, K. Woodbridge, and C. Roberts, Phys. Rev. B 41, 1090 (1990).

[80] K. S. Kim, G. M. Yang, H. W. Shim, K. Y. Lim, E.-K. Suh, and H. J. Lee, J. Appl. Phys. 82, 5103 (1997).

[81] K. S. Kim, G. M. Yang, and H. J. Lee, J. Vac. Sci. Technol. A 16, 2663 (1998).

[82] C. M. N. Mateo, A. T. Garcia, F. R. M. Ramos, K. I. Manibog, and A. A. Salvador, J. Appl. Phys. 101, 73519 (2007).

[83] C. M. N. Mateo, J. J. Ibañez, J. G. Fernando, J. C. Garcia, K. Omambac, R. B. Jaculbia, M. Defensor, and A. A. Salvador, J. Appl. Phys. 104, 103537 (2008).

[84] B. Fluegel, A. V. Mialitsin, D. A. Beaton, J. L. Reno, and A. Mascarenhas, Nat. Commun. 6, 7136 (2015).

[85] Y.-S. Lee, T. B. Norris, M. Kira, F. Jahnke, S. W. Koch, G. Khitrova, and H. M. Gibbs, Phys. Rev. Lett. 83, 5338 (1999).

[86] C. Weisbuch, M. Nishioka, A. Ishikawa, and Y. Arakawa, Phys. Rev. Lett. 69, 3314 (1992).

[87] M. Koch, J. Shah, and T. Meier, Phys. Rev. B 57, R2049 (1998).

[88] J. Kasprzak, M. Richard, S. Kundermann, A. Baas, P. Jeambrun, J. M. J. Keeling, F. M. Marchetti, M. H. Szymańska, R. André, J. L. Staehli, V. Savona, P. B. Littlewood, B. Deveaud, and L. S. Dang, Nature 443, 409 (2006).

[89] A. P. D. Love, D. N. Krizhanovskii, D. M. Whittaker, R. Bouchekioua, D. Sanvitto, S. A. Rizeiqi, R. Bradley, M. S. Skolnick, P. R. Eastham, R. André, and L. S. Dang, Phys. Rev. Lett. 101, (2008).

[90] V. Kohnle, Y. Léger, M. Wouters, M. Richard, M. T. Portella-Oberli, and B. Deveaud, Phys. Rev. B 86, (2012).

[91] S. Kundermann, M. Saba, C. Ciuti, T. Guillet, U. Oesterle, J. L. Staehli, and B. Deveaud, Phys. Rev. Lett. 91, (2003).

[92] V. Ardizzone, P. Lewandowski, M. H. Luk, Y. C. Tse, N. H. Kwong, A. Lücke, M. Abbarchi, E. Baudin, E. Galopin, J. Bloch, A. Lemaitre, P. T. Leung, P. Roussignol, R. Binder, J. Tignon, and S. Schumacher, Sci. Rep. 3, (2013).

[93] P. Borri, W. Langbein, U. Woggon, A. Esser, J. R. Jensen, and J. M. Hvam, Semicond. Sci. Technol. 18, S351 (2003).

[94] M. Saba, F. Quochi, C. Ciuti, U. Oesterle, J. L. Staehli, B. Deveaud, G. Bongiovanni, and A. Mura, Phys. Rev. Lett. 85, 385 (2000).

[95] G. Khitrova, H. M. Gibbs, F. Jahnke, M. Kira, and S. W. Koch, Rev. Mod. Phys. 71, 1591 (1999).

[96] H. M. Gibbs, G. Khitrova, and S. W. Koch, Nat. Photonics 5, 273 (2011).

[97] S. Christopoulos, G. B. H. von Högersthal, A. J. D. Grundy, P. G. Lagoudakis, A. V. Kavokin, J. J. Baumberg, G. Christmann, R. Butté, E. Feltin, J.-F. Carlin, and N. Grandjean, Phys. Rev. Lett. 98, (2007).

[98] P. Bhattacharya, T. Frost, S. Deshpande, M. Z. Baten, A. Hazari, and A. Das, Phys. Rev. Lett. 112, (2014).

[99] I. Shelykh, Y. Rubo, G. Malpuech, D. Solnyshkov, and A. Kavokin, Phys. Rev. Lett. 97, (2006).

[100] G. Roumpos, M. D. Fraser, A. Löffler, S. Höfling, A. Forchel, and Y. Yamamoto, Nat. Phys. 7, $129(2011)$. 
[101] A. Amo, J. Lefrère, S. Pigeon, C. Adrados, C. Ciuti, I. Carusotto, R. Houdré, E. Giacobino, and A. Bramati, Nat. Phys. 5, 805 (2009).

[102] D. Sanvitto, F. M. Marchetti, M. H. Szymańska, G. Tosi, M. Baudisch, F. P. Laussy, D. N. Krizhanovskii, M. S. Skolnick, L. Marrucci, A. Lemaître, J. Bloch, C. Tejedor, and L. Viña, Nat. Phys. 6, 527 (2010).

[103] N. Takemura, S. Trebaol, M. Wouters, M. T. Portella-Oberli, and B. Deveaud, Nat. Phys. 10, 500 (2014).

[104] M. Sich, D. N. Krizhanovskii, M. S. Skolnick, A. V. Gorbach, R. Hartley, D. V. Skryabin, E. A. Cerda-Méndez, K. Biermann, R. Hey, and P. V. Santos, Nat. Photonics 6, 50 (2011).

[105] J. M. Blatt, K. W. Böer, and W. Brandt, Phys. Rev. 126, 1691 (1962).

[106] D. Snoke, Science 298, 1368 (2002).

[107] G. Malpuech, A. Kavokin, and F. P. Laussy, Phys. Status Solidi A 195, 568 (2003).

[108] F. Laussy, I. Shelykh, G. Malpuech, and A. Kavokin, Phys. Rev. B 73, (2006).

[109] H. Deng, H. Haug, and Y. Yamamoto, Rev. Mod. Phys. 82, 1489 (2010).

[110] F. Albert, K. Sivalertporn, J. Kasprzak, M. Strauß, C. Schneider, S. Höfling, M. Kamp, A. Forchel, S. Reitzenstein, E. A. Muljarov, and W. Langbein, Nat. Commun. 4, 1747 (2013).

[111] T. Baars, G. Dasbach, M. Bayer, and A. Forchel, Phys. Rev. B 63, (2001).

[112] A. Baas, J. Karr, H. Eleuch, and E. Giacobino, Phys. Rev. A 69, (2004).

[113] Peter Hamm and Martin Zanni, Concepts and Methods of 2D Infrared Spectroscopy, 1 st ed. (2011).

[114] Robert Boyd, Nonlinear Optics, 3rd ed. (Academic Press, 2008).

[115] Guang S. He and Song H. Liu, Physics of Nonlinear Optics (World Scientific, Singapore, 1999).

[116] Shaul Mukamel, Principles of Nonlinear Optical Spectroscopy (Oxford University Press, 1999).

[117] S. Mukamel and R. F. Loring, JOSA B 3, 595 (1986).

[118] K. B. Ferrio and D. G. Steel, Phys Rev Lett 80, 786 (1998).

[119] M. E. Donovan, A. Schülzgen, J. Lee, P.-A. Blanche, N. Peyghambarian, G. Khitrova, H. M. Gibbs, I. Rumyantsev, N. H. Kwong, R. Takayama, Z. S. Yang, and R. Binder, Phys Rev Lett 87, (2001).

[120] A. G. V. Spivey and S. T. Cundiff, J Opt Soc Am B 24, 664 (2007).

[121] K. Stone, D. Turner, K. Gundogdu, S. Cundiff, and K. Nelson, Acc. Chem. Res. 42, 1452 (2009).

[122] T. Guenther, C. Lienau, T. Elsaesser, M. Glanemann, V. M. Axt, T. Kuhn, S. Eshlaghi, and A. D. Wieck, Phys. Rev. Lett. 89, (2002).

[123] G. Nardin, T. M. Autry, G. Moody, R. Singh, H. Li, and S. T. Cundiff, J. Appl. Phys. 117, 112804 (2015).

[124] https://en.wikipedia.org/wiki/Kerr-lens_modelocking, Wikipedia (n.d.).

[125] J.-C. Diels and W. Rudolph, Ultrashort Laser Pulse Phenomena: Fundamentals, Techniques, and Applications on a Femtosecond Time Scale, 2nd ed (Elsevier/Academic Press, Amsterdam; Boston, 2006).

[126] P. W. Milonni and J. H. Eberly, Lasers Physics (John Wiley \& Sons, Hoboken, N.J, 2010).

[127] Joseph T. Verdeyen, Laser Electronics, second (Prentice hall, Englewood Cliffs, New Jersey, 1989).

[128] D. T. F. Marple, J. Appl. Phys. 35, 1241 (1964).

[129] S. Adachi, J Appl Phys 58, R1 (1985). 
[130] M. Grundmann, The Physics of Semiconductors, 2nd ed. (Springer-Verlag, 2010).

[131] S. T. Cundiff, Opt. Express 16, 4639 (2008).

62Ioffe Physico-Technical Intitute (2016), http://www.ioffe.ru/SVA/NSM/Semicond/GaAs/index.html.

[133] A. E. Almand-Hunter, H. Li, S. T. Cundiff, M. Mootz, M. Kira, and S. W. Koch, Nature 506, 471 (2014).

[134] J. P. Eisenstein and A. H. MacDonald, Nature 432, 691 (2004).

[135] Carson D. Jeffries, Science 189, 955 (1975).

[136] D. Bressanini, M. Mella, and G. Morosi, Phys. Rev. A 57, 4956 (1998).

[137] Y. Z. Hu, S. W. Koch, M. Lindberg, N. Peyghambarian, E. L. Pollock, and F. F. Abraham, Phys. Rev. Lett. 64, 1805 (1990).

[138] S. T. Cundiff, JOSA B 29, A69 (2012).

[139] B. L. Wilmer, D. Webber, J. M. Ashley, K. C. Hall, and A. D. Bristow, Phys. Rev. B 94, 75207 (2016).

[140] K.-H. Goetz, D. Bimberg, H. Jürgensen, J. Selders, A. V. Solomonov, G. F. Glinskii, and M. Razeghi, J. Appl. Phys. 54, 4543 (1983).

[141] Sajal Paul, J. B. Roy, and P. K. Basu, 69, 827 (1991).

117InGaAs (2016), http://www.ioffe.ru/SVA/NSM/Semicond/GaInAs/bandstr.html.

[143] E. S. Lee, Light-Exciton Coupling in Semiconductor Micro-and Nano-Structures, The University of Arizona, 2001.

[144] D. V. Wick, Nonlinear Optical Processes in Semiconductor Microcavities and Sodium Vapor, The University of Arizona, 1997.

[145] O. Lyngnes, Optical Properties of Semiconductor Nano-Structures and Photoactive Yellow Protein, The University of Arizona, 1997.

[146] Eric Karl Lindmark, Erbium Doped GaAs/AlGaAs Quantum Wells and Normal Mode Coupling of Semiconductor Microcavities, The University of Arizona, 1997.

[147] M. V. Karachevtseva, A. S. Ignat'ev, V. G. Mokerov, G. Z. Nemtsev, V. A. Strakhov, and N. G. Yaremenko, Semiconductors 28, 691 (1999).

[148] J. P. Reithmaier, Semicond. Sci. Technol. 23, 123001 (2008).

[149] M. J. Joyce, Z. Y. Xu, and M. Gal, Phys. Rev. B 44, 3144 (1991).

[150] R. Atanasov, F. Bassani, A. D'Andrea, and N. Tomassini, Phys. Rev. B 50, 14381 (1994).

[151] J. Barnes, J. Nelson, K. W. J. Barnham, J. S. Roberts, M. A. Pate, R. Grey, S. S. Dosanjh, M. Mazzer, and F. Ghiraldo, J. Appl. Phys. 79, 7775 (1996).

[152] W. S. Chi and Y. S. Huang, Semicond. Sci. Technol. 10, 127 (1995).

[153] K. J. Vahala, Nature 424, 839 (2003).

[154] E. M. Purcell, H. C. Torrey, and R. V. Pound, Phys. Rev. 69, 37 (1946).

[155] E. T. Jaynes and F. W. Cummings, Proc. IEEE 51, 89 (1963).

[156] J. J. Sanchez-Mondragon, N. B. Narozhny, and J. H. Eberly, Phys. Rev. Lett. 51, 550 (1983).

[157] E. Yablonovitch, Phys. Rev. Lett. 58, 2059 (1987).

[158] E. S. Lee, S. Park, P. Brick, C. Ell, C. Spiegelberg, H. M. Gibbs, G. Khitrova, D. G. Deppe, and D. L. Huffaker, in Dir. Quantum Opt. (Springer, 2001), pp. 230-237.

[159] G. E. Jellison, Opt. Mater. 1, 151 (1992).

[160] R. E. Fern, J. Appl. Phys. 42, 3499 (1971).

[161] Ricky Gibson, (2013).

[162] H. J. Kimble, Phys. Scr. 1998, 127 (1998).

[163] G. Khitrova, H. M. Gibbs, M. Kira, S. W. Koch, and A. Scherer, Nat. Phys. 2 , 81 (2006). 
[164] V. Savona and C. Weisbuch, Phys. Rev. B 54, 10835 (1996).

[165] S. Pau, G. Björk, J. Jacobson, H. Cao, and Y. Yamamoto, Phys. Rev. B 51, 14437 (1995).

[166] A. Tredicucci, Y. Chen, V. Pellegrini, M. Börger, L. Sorba, F. Beltram, and F. Bassani, Phys. Rev. Lett. 75, 3906 (1995).

[167] R. Houdré, J. L. Gibernon, P. Pellandini, R. P. Stanley, U. Oesterle, C. Weisbuch, J. O'Gorman, B. Roycroft, and M. Ilegems, Phys. Rev. B 52, 7810 (1995).

[168] M. S. Skolnick, T. A. Fisher, and D. M. Whittaker, Semicond. Sci. Technol. 13, 645 (1998).

[169] J. J. Hopfield, Phys. Rev. 112, 1555 (1958).

[170] C. Ciuti, P. Schwendimann, and A. Quattropani, Semicond. Sci. Technol. 18, S279 (2003).

[171] E. Tokunaga, A. L. Ivanov, S. V. Nair, and Y. Masumoto, Phys. Rev. B 63, (2001).

[172] T. P. Humphreys, J. B. Posthill, K. Das, C. A. Sukow, R. J. Nemanichi, N. R. Parikh, and A. Majeed, Jpn. J. Appl. Phys. 28, L1595 (1989).

[173] E. Grilli, M. Guzzi, R. Zamboni, and L. Pavesi, Phys. Rev. B 45, 1638 (1992).

[174] A. S. Cooper, Acta Crystallogr. 15, 578 (1962).

[175] M. Lucht, M. Lerche, H.-C Wille, Yu. V. Shvyd'ko, H.D. Rüter, E. Gerdau, and P. Becker, J. Appl. Crystallogr. 36, 141 (2003).

[176] C. F. Klingshirn, Semiconductor Optics, 3rd ed. (Springer-Verlag, 2010).

[177] A. Gavini and M. Cardona, Phys. Rev. B 1, 672 (1970).

[178] Y. Sun, S. E. Thompson, and T. Nishida, J. Appl. Phys. 101, 104503 (2007).

[179] I. Vurgaftman, J. R. Meyer, and L. R. Ram-Mohan, J. Appl. Phys. 89, 5815 (2001).

[180] L. Yang and S. Mukamel, J. Phys. Condens. Matter 20, 395202 (2008).

[181] G. W. 't Hooft, W. A. J. A. van der Poel, L. W. Molenkamp, and C. T. Foxon, Phys. Rev. B 35, 8281 (1987).

[182] G. Moody, R. Singh, H. Li, I. A. Akimov, M. Bayer, D. Reuter, A. D. Wieck, A. S. Bracker, D. Gammon, and S. T. Cundiff, Phys. Status Solidi B 250, 1753 (2013).

[183] C. N. Borca, T. H. Zhang, X. Q. Li, and S. T. Cundiff, Chem Phys Lett 416, 311 (2005).

[184] Y. Z. Hu, R. Binder, S. W. Koch, S. T. Cundiff, H. Wang, and D. G. Steel, Phys Rev B 49, 14382 (1994).

[185] D. Webber, L. Hacquebard, X. Liu, M. Dobrowolska, J. K. Furdyna, and K. C. Hall, Appl. Phys. Lett. 107, 142108 (2015).

[186] D. J. Dunstan, J. Mater. Sci. Mater. Electron. 8, 337 (1997).

[187] J. J. Olivero and R. L. Longbothum, J. Quant. Spectrosc. Radiat. Transf. 17, 233 (1977).

[188] S. B. Nam, D. C. Reynolds, C. W. Litton, R. J. Almassy, T. C. Collins, and C. M. Wolfe, Phys. Rev. B 13, 761 (1976).

[189] J. P. Prineas, C. Ell, E. S. Lee, G. Khitrova, H. M. Gibbs, and S. W. Koch, (2001).

[190] D. M. Whittaker, P. Kinsler, T. A. Fisher, M. S. Skolnick, A. Armitage, A. M. Afshar, M. D. Sturge, and J. S. Roberts, Phys. Rev. Lett. 77, 4792 (1996).

[191] C. Ell, J. Prineas, T. R. Nelson Jr, S. Park, H. M. Gibbs, G. Khitrova, S. W. Koch, and R. Houdré, Phys. Rev. Lett. 80, 4795 (1998).

[192] W. Langbein, J. M. Hvam, M. Umlauff, H. Kalt, B. Jobst, and D. Hommel, Phys. Rev. B 55, R7383 (1997).

[193] Giacobino, Elisabeth, JP. Karr, G. Messin, H. Eleuch, and A. Baas, Comptes Rendus Phys. 3, 41 (2002).

[194] D. Birkedal, V. G. Lyssenko, J. M. Hvam, and K. E. Sayed, Phys Rev B 54, 14250 (1996).

[195] P. G. Kevrekidis, G. Theocharis, D. J. Frantzeskakis, and B. A. Malomed, Phys. Rev. Lett. 90, (2003). 
[196] E. Timmermans, P. Tommasini, M. Hussein, and A. Kerman, Phys. Rep. 315, 199 (1999).

[197] Brian L. Wilmer, Proc. Soc. Photogr. Instrumentaion Eng. 9746, (2016).

[198] L. Yang and S. Mukamel, Phys. Rev. Lett. 100, (2008).

[199] L. Yang, T. Zhang, A. D. Bristow, S. T. Cundiff, and S. Mukamel, J. Chem. Phys. 129, 234711 (2008). 


\section{Appendix}

\section{A.1 Alignment Procedure}

\section{A.1.1 Bottom Deck}

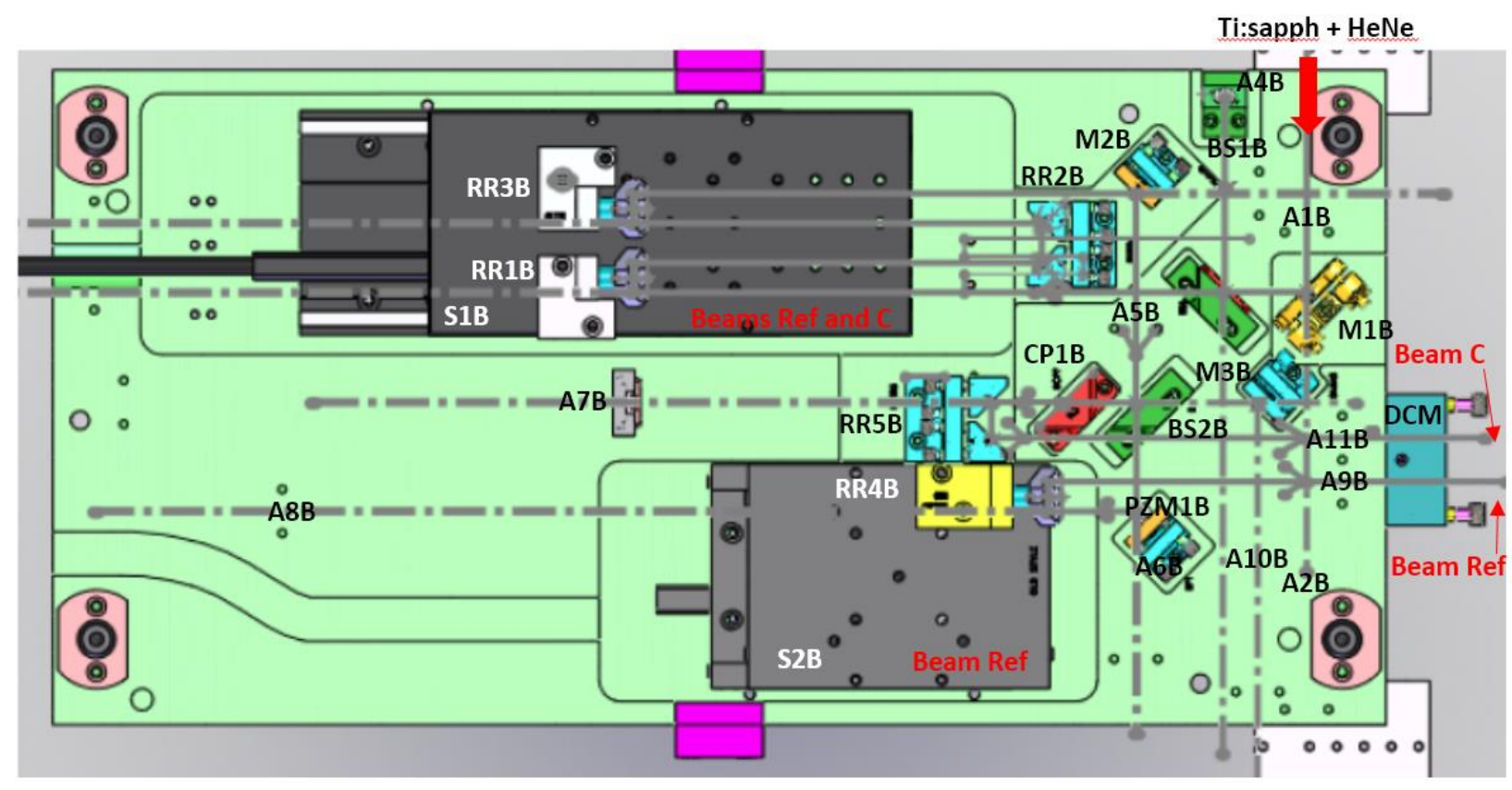

Figure 74 MONSTR Bottom deck alignment schematic

1. Align beams into the bottom deck so that they pass through apertures at position $A 1 B$ and $\mathrm{A} 2 \mathrm{~B}$

2. Insert Newport mirror M1B.

3. Assemble two-axis translation with razor blades to ensure that beam reflected from M1B is parallel to the motion of delay stage S1B in both transverse directions (horizontal and vertical).

a. Mount razor blade on stage position to block the beam approximately $50 \%$. Use a detector to measure the remaining light. Move the stage correcting the pointing until the reading on the detector does not change with motion of the stages.

4. Secure beam splitter BS1B to the bottom deck. The reflecting surface should be on the beam entrance side to match compensator plate positions. Align reflection onto the center of telescope mirror TM1 position. Mount TM1 and adjust BS1B and TM1 so that the light is centered on an aperture on the periscope extension. 
5. Build and mount the corner-cube retroreflector RR1B.

a. Attach the corner cube retroreflector to its collar using the PLX RR squaring fixture. The orientation is important, because the retroreflector mirror has been modified to sit closer to the top surface of the stage. Attach the collar first with the two screws at the back. Attach the corner cube with a since screw from the back, leave loose. Adjust the two long screws on the side to lock the azimuthal orientation, as close as possible to the correct orientation. The flattened edge should be towards the bottom of the squaring fixture. Once the locking screws are right, tighten the single mounting screw on the back of the corner cube. Now the collar should be securely attached to the corner cube and the assembly can be removed from the fixture.

b. Mount the collared corner cube into its stage mounting (BLR9) and attach to the stage. Ensure that positions of the three laser spots are symmetric and are not hitting any of the retroreflector seams.

6. The light reflected from RR1B should be centered on the input mirror of the static retroreflector RR2B. Adjust the beam across to the center of the output mirror of RR2B. Ensure the beam stays at the same height.

7. Use the two-axis translation with razor blades from step 3 to ensure that the reflected beam from RR2B is parallel to the motion of delay stage S1B in both transverse directions (horizontal and vertical).

8. Build and mount the corner-cube retroreflector RR3B following the procedure in step 5. The stage mounting is BLL10. Once RR3B is mounted double check the overall stage alignment and walk off through aperture position A4B. Correct using exit mirror of RR2B.

9. Secure mirror M2B to the bottom deck.

10. Align the reflection from M2B through the A5B and A6B. Tap the corner cube mount of RR3B, when it is loosely secured to the BLL10, to center the beam on A5B. Set the direction through $\mathrm{A6B}$ using the direction actuators on $\mathrm{M} 2 \mathrm{~B}$. Use all three actuators to align the depth position on $\mathrm{M} 2 \mathrm{~B}$ and provide additional lateral translation on $\mathrm{A} 5 \mathrm{~B}$. 
Remember to tighten the mounting screws on RR3B. Finally check the beam pointing and stage walk after A6B.

11. Secure BS2B on the bottom deck. The reflecting surface should be on the beam entrance side to match compensator plate positions. Align the reflection through A7B.

12. Mount the compensator plate CP1B and secure on bottom deck.

13. Mount piezoelectric transducer mirror PZM1B. Initially align the reflection through A8B. Then use the two-axis translation with razor blades from 3 to ensure that the reflected beam from PZM1B is parallel to the motion of delay stage S2B in both transverse directions (horizontal and vertical). Maintain approximate alignment through $A 8 B$ by adjusting all three actuators.

14. Build and mount the corner-cube retroreflector RR4B following the procedure in 5 . The stage mounting is BS8. Align the reflection to go through A9B. Using a razor blade and detector align the beam walk using PZM1B.

15. Mount the 2-inch dichroic mirror DCM. Align the back reflection of path through stage S2B so that it retraces its own path.

16. Mount M3B to deflect the returning light out through the side of the MONSTR. Aligning the reflection from $\mathrm{M} 3 \mathrm{~B}$ through $\mathrm{A} 10 \mathrm{~B}$.

17. Mount RR5B. Initially use both mirrors to align the beam through A11B. Align the beam to propagate parallel to the beam passing through path with S2B. They should propagate over a long distance parallel about 1 inch apart.

18. To get parallel beams with the correct spacing and interference fringes at $A 10 B$ you may need to adjust all 6 actuators on RR5B. Only make minor adjustments to the DCM. Do not adjust anything along the path with $\mathrm{S} 2 \mathrm{~B}$, since it acts as a reference for aligning the path through RR5B. 


\section{A.1.2 Top Deck}

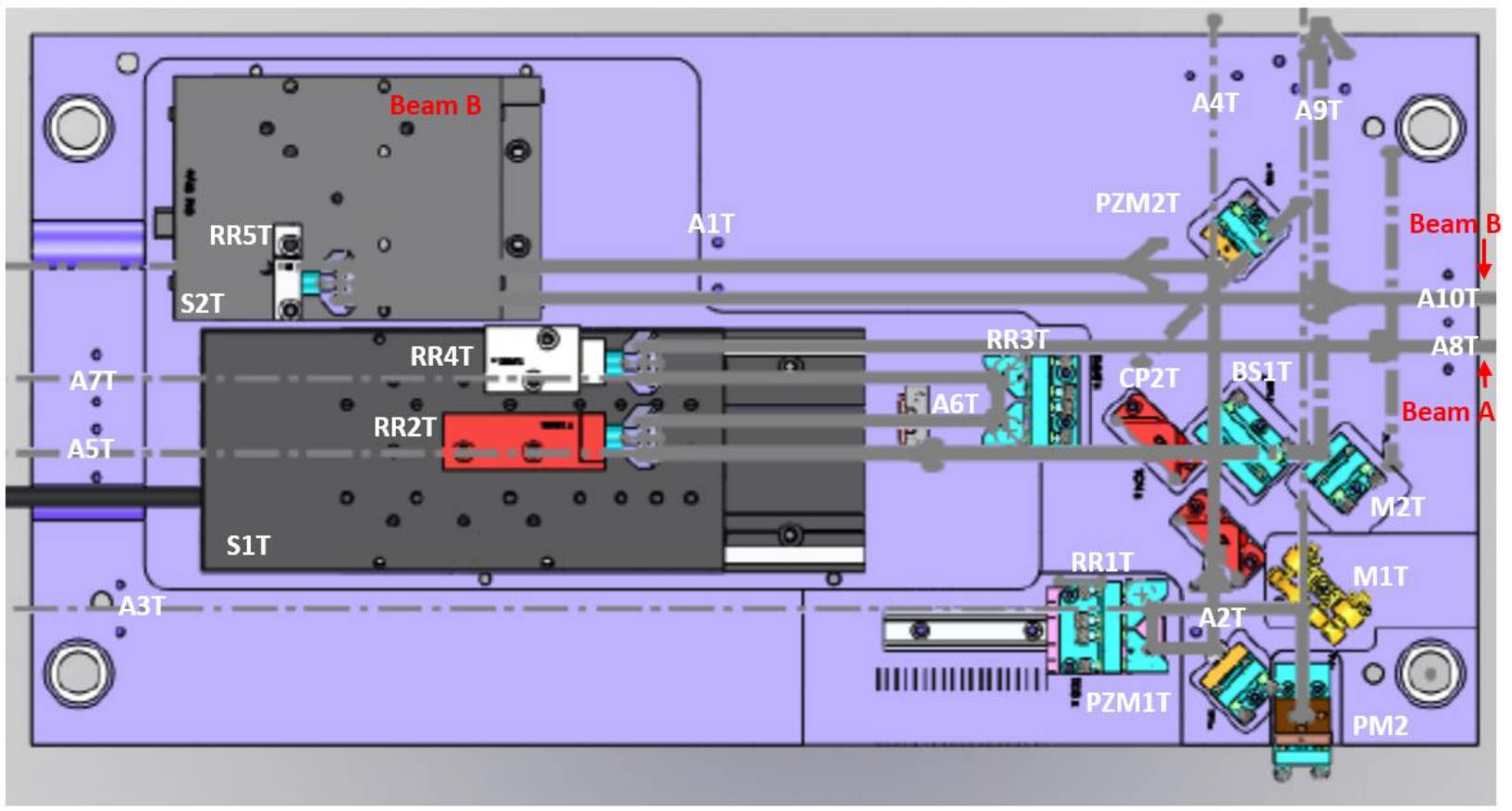

Figure 75 MONSTR top deck alignment schematic

center of the PM2 position and the aperture position A1T. Use a business card and mark the height of A1T for height reference at the beginning of the beam path on the deck.

1. Secure the Newport mirror mount M1T. Align the reflection through A2T and A3T.

2. Secure RR1T to the top deck. Align the reflection beam spot so that it does not move on a card placed some distance from the deck.

3. Mount the PZT transducer mirror into PZM1T and secure on top deck. Align the reflection through the second set of holes at A2T position and through A4T. Note that the mounted aperture will not work at A2T, so make an alignment card.

4. Secure CP1T, CP2T and BS1T to the top deck. Initially align the reflection from BS1T through A5T.

5. Secure S1T to the top deck. Use the two-axis translation with razor blades from Bottom Deck Alignment 3 to ensure that the reflected beam from BS1T is parallel to the motion of delay stage S1T in both transverse directions (horizontal and vertical). 
6. Assemble RR2T using the procedure in Bottom Deck Alignment 5. Secure RR2Tand RR3T to top deck. Ensure that the reflection from RR2T impinges the center of the input mirror on RR3T. If it does not adjust the position of the corner cube in the mount, through aperture position A6T. If required you may have to adjust the depth of BS1T using all three actuators.

7. Align the output from RR3T to A7T. Then use the two-axis translation with razor blades from Bottom Deck Alignment 3 to ensure that the reflected beam from RR3T is parallel to the motion of delay stage S1T in both transverse directions (horizontal and vertical).

8. Assemble RR4T using the procedure in Bottom Deck Alignment 5 and secure it to top deck. Align the reflection through aperture position A8T and ensure that stage motion does not cause the beam to walk.

9. Assemble PZM2T and secure to the top deck. Align reflection through A9T. Then use the two-axis translation with razor blades from Bottom Deck Alignment 3 to ensure that the reflected beam from PZM2T is parallel to the motion of delay stage S2T in both transverse directions (horizontal and vertical).

10. Assemble RR5T. Note that there is a smaller collar for the retroreflector. Secure to S2T. Adjust the position so that the beam is symmetric on the retroreflector and that the reflection passes through $\mathrm{A} 10 \mathrm{~T}$.

11. Attach DCM. Do not adjust its tilt from alignment of the bottom deck. Make the beams from the two arm overlap at the output port from $\mathrm{M} 2 \mathrm{~T}$, once it has been mounted. Do not adjust the beam path through S2T, since it acts as a reference for the beam path through S1T.

12. We had to perform the alignment for S2T first, then for S1T second. This lead to an angular divergence between the beams exiting the MONSTR with zero motion of the beams as the stages where translated. The way to correct the angle is to rotate BS1T to make the beams parallel. This introduces motion in to the beam which can be removed by rotation of S1T. Then adjust the beam onto aperture A8T using RR4T. Repeat these steps as required. 


\section{A.1.3 Assembly}

1. Ensure that before closing the two decks that the out beams for the individual decks have exactly the correct height from the decks, i.e. $1 / 2$ inch. This is important because there may be no way to adjust the relative separation of the top and bottom deck.

2. Secure bottom deck to optical table. Align beams through setup. Do not connect the DCM.

3. Attach the PM2 to the top deck.

4. Place top deck on to bottom deck inverted. Use the four jack screws and the protection pads to protect the surface of the lower. Use a level to keep the top deck from binding on the bushels. Lower the top deck using the jack screws $1 / 8$ of a turn. If the top deck binds, then lift it again until it is level.

5. Before the top deck is full lowered secure the side supports and the DCM to the bottom deck.

6. When the top deck is fully lowered secure the side supports and DCM to the top deck. Also, secure the top deck with the top deck locking nuts.

7. The weight of the closed MONSTR on the padded feet will compress them slightly, therefore the input alignment will need to be adjusted. Using the HeNe beam and adjusting only the HeNe laser's input mirrors, use two-axis translation and the razor blades to ensure the reference beam is centered on the aperture positon $A 9 B$ and has zero movement with respect to the stage movement.

8. Adjust the Ti:Sapph's input mirrors to bring it to the HeNe alignment. Use an iris at the input port of the MONSTR and an iris distantly placed after the MONSTR output.

9. Since the deck were aligned separately, the periscope mirrors (PM1 and PM2) and M1T are not inputting the beams into the top deck properly. Adjust PM1 and PM2 to correct any vertical angle and translation errors. Use razor blades and two-axis translation and either $\mathrm{A}$ or $\mathrm{B}$ to adjust the mirrors.

10. Use PM2 and M1T to correct any horizontal angular and translation errors.

11. Adjustment of BS1T should not be necessary. If it is, use two axis translation to make the beam parallel to the S1T travel and use RR2T and RR4T to center the beam on A8T. The 
use PZM2T and razor blades with two axis ration to make the beam parallel to S2T and adjust RR5T to center the beam on A10T.

12. The DCM may need to be purposefully misaligned if the alignment is good because the retroreflections disrupts the oscillator's modelocking.

\section{A.2 Post Alignment Optimization}

The alignment will drift overtime or there may have been errors in a previous alignment. If not too extreme, the follow procedure will align the beams optically perfectly. Any walk of the beams afterwards is due to the stages not being bolted to the decks mutually parallel and perpendicular to the front MONSTR face.

1. First ensure the HeNe and Ti:Sapph beams are collinear by removing the dichroic mirror and placing an Iris position at the farthest possible point from the MONSTR. Determine which laser hast the most error and using its input mirrors into the MONSTR, bring its alignment to the other laser. Note, the Ti:Sapph's input mirrors tend to drift more than the HeNe's.

2. Using the reference beam (HeNe or Ti:sapph) MONSTR output and two-axis translation with razor blades, adjust PZM1B until the beam is parallel to S2B.

3. Adjust RR4B to center the reference beam on iris position A9B. Remount the dichroic mirror and adjust until the retroreflection HeNe laser.

4. Place a card in front of the bottom deck interferometer output at A6B. Using RR5B adjust beam $C$ until it is both centered on Iris position A11B and giving circular interference fringes at A6B. The retroreflection should also go back to the HeNe.

5. Place a card in front of the top and interdeck interferometer output at A1T and A10B. Adjust PM2 to optimize interference of A with C or Ref. Then use RR2T and 4T to center A on A8T. This choice of optic to align assumes that BS1T is good and need not be adjusted.

6. Adjust PZM2T to optimize the interference of $B$ with $A$ on the top deck pattern at A1T and $B$ with $C$ or ref at the interdeck interferometer. Use RR5T to center the output on A10T. 
7. All four beams should be nearly perfectly parallel now. This can be verified by using the replica focus objective and Ti:sapph to see the beams focus at the same point. Do a fine tweaking of the Ti:sapph if needed.

8. Finally, the dichroic may have to be slightly misaligned if the alignment is good because the retroflection from the Ti:Sapph can interrupt the modelocking.

\section{A.3 Replica Focus Alignment}

1. With autocorrelation complete, put a glass window into beam path. Make an alignment card with four holes to check that the box is reflected back on center.

2. The window will shift the whole focus spatially away from the MONSTR. Therefore, you'll have to move the BBO farther to check that the autocorrelation by eye is unchanged.

a. If you're checking this from a previous alignment, you can avoid moving the glass' post by removing the glass itself from the mount.

3. Place turning mirror to reflect whole box 90 degrees towards objective. Check this is with card taped to a post. Mark the card (a diagonal?) and use the holes on the table as references.

4. Remove mirror from corner cube and put the positioning apparatus in place such that the cube in the center of the beam. Make sure it stays roughly centered as you move the box along the beam axis. Tape a card onto box for this check.

5. Put $10 x$ objective into cube and put into focus until four beams comes out the back. Align with the center box so that (out box is reflection from second glass face). Again, use business card to make sure the box doesn't walk.

a. As you scan through the focus, the box and beams should remain symmetric, move the objective until it is...

6. Increase to $20 x$, and repeat up to $50 x$ objective.

7. At 50x, fringes should be visible by placing a magnifying (I used $2.5 \mathrm{~cm}$ ) lens in the beam. You'll have to use either an IR viewer or IR card to see the fringes. Put a paper target on a cart and position it at some distance from the table to make the fringes large.

8. Once optimized, replace corner cube mirror and center on CCD camera 
9. Rotation the CCD camera so the vertical (horizontal) fringes are vertical (horizontal) on CCD output.

\section{A.4 Initialization}

1. Turn MIRA key to on then immediately press the standby button on the MIRA'S chiller to turn it on. This laser will take $\sim .5$ hours to stabilize.

2. Turn the HeNe laser key to on. This laser will take $\sim 1$ hour to stabilize.

3. Switch on the oscilloscope, power strip with loop filter high voltage power supplies, loop filters, the four error signal detectors, external interferometer detector amplifier, the AeroTech stage driver box.

4. Turn on MONSTR computer and run the Master_Control, CCF_FFT, and Replica_focus Labview VIs.

5. On Master_Control.vi: the READY Boolean will be set after each software command is executed and completed. Choose all stages by setting the Booleans for each to true, ENGAGE the stages and HOME the stages. Set the speed of each stage to $10000 \mu \mathrm{m} / \mathrm{s}$ GOTO the desired absolute position of each stage. The stages are now coarsely at the desired positions.

6. Lower all of the speeds to $<100 \mu \mathrm{m} / \mathrm{s}$ and GOTO again. The stages are now within $<.001$ $\mu \mathrm{m}$ of their desired position

\section{A.5 Autocorrelation}

Run the replica focus program and unblock the bottom deck beam shutters and tra shutter. Place a card in the reference retroreflector delay stage and in front of the sample if present to avoid saturating the spectrometer.

1. Open C, Ref, and Tracer shutters. If the beams are not spatially overlapped there is a misalignment. This must be fixed before proceeding. Observe interference between $\mathrm{C}$ and Ref at the replica focus. Adjust the exposure time so as not fill but not saturate the detector. 
2. Move Ref pulse in time by moving stage S2B until the interference is coarsely optimized. It should be similar to that of Figure 76.

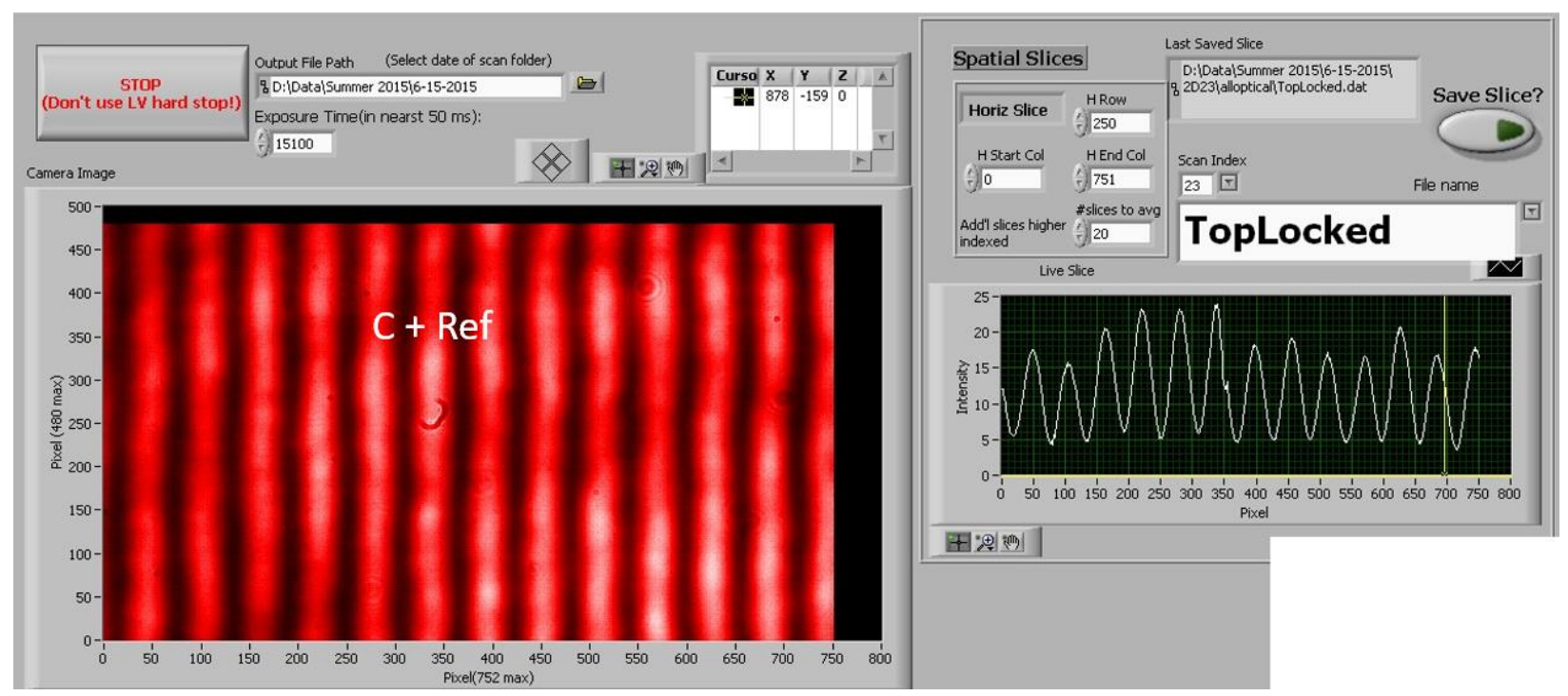

Figure 76 Good interference pattern from reference and $C$ beams and the corresponding onedimensional slice

3. Select the Boolean to scan Ref through $C$ centered about the position found in the previous step. Set the scan speed to $.4 \mu \mathrm{m} / \mathrm{s}$ and the scan distance to $20 \mu \mathrm{m}$. Hit SCAN. Ref will now be scanned in time through temporal overlap with $\mathrm{C}$ by moving stage S2B. Figure 77 shows a partially finished autocorrelation. 


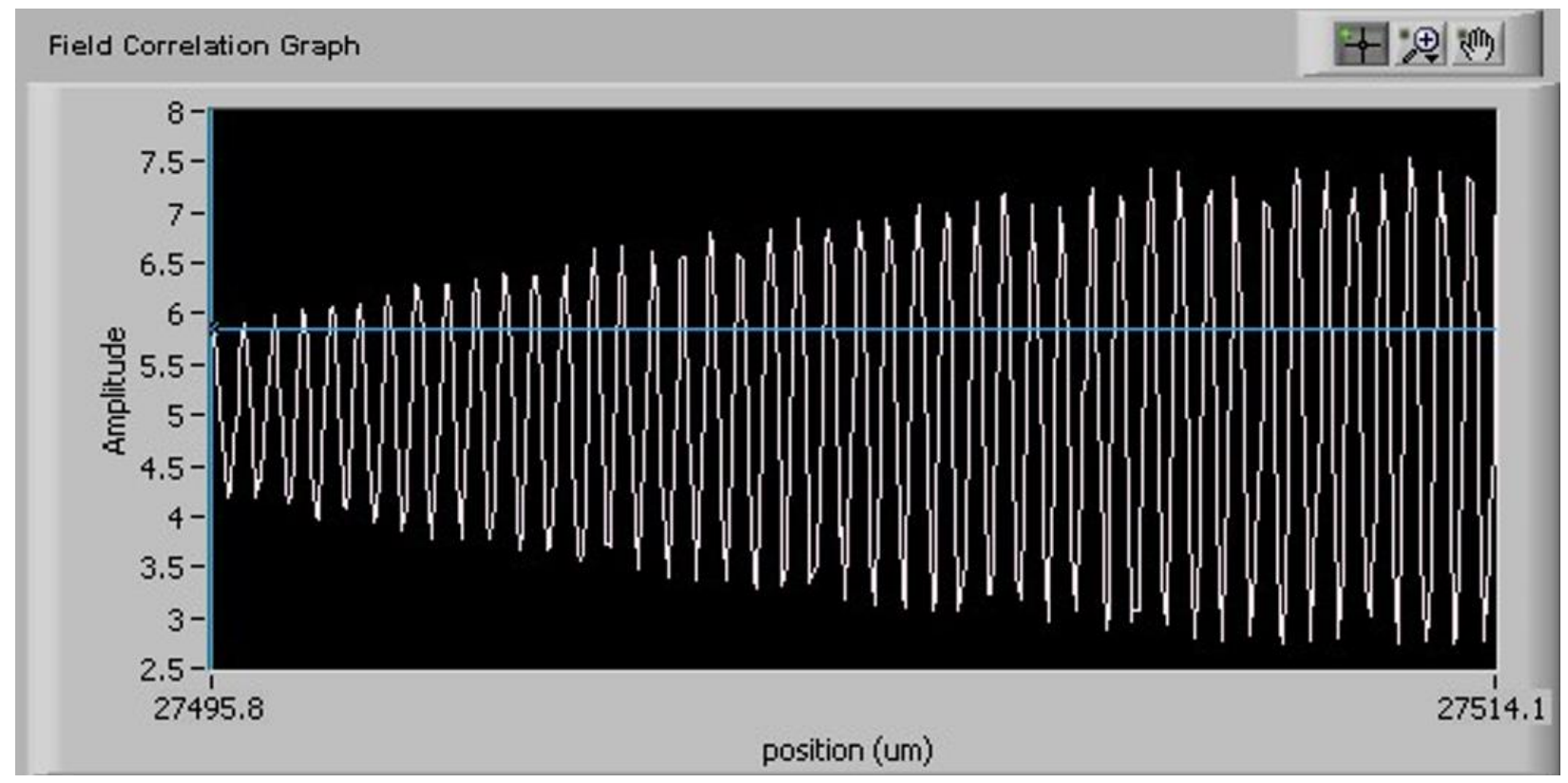

Figure 77 Partially finished autocorrelation

4. Observe autocorrelation results. Select the center of the autocorrelation with the curser and record the horizontal coordinate and input this coordinate into the Master_Control position for Ref and hit GOTO. Pulses Reference and C are now at zero time delay with respect to one another. Close the shutters. Figure 78 shows a finished autocorrelation with center picked.

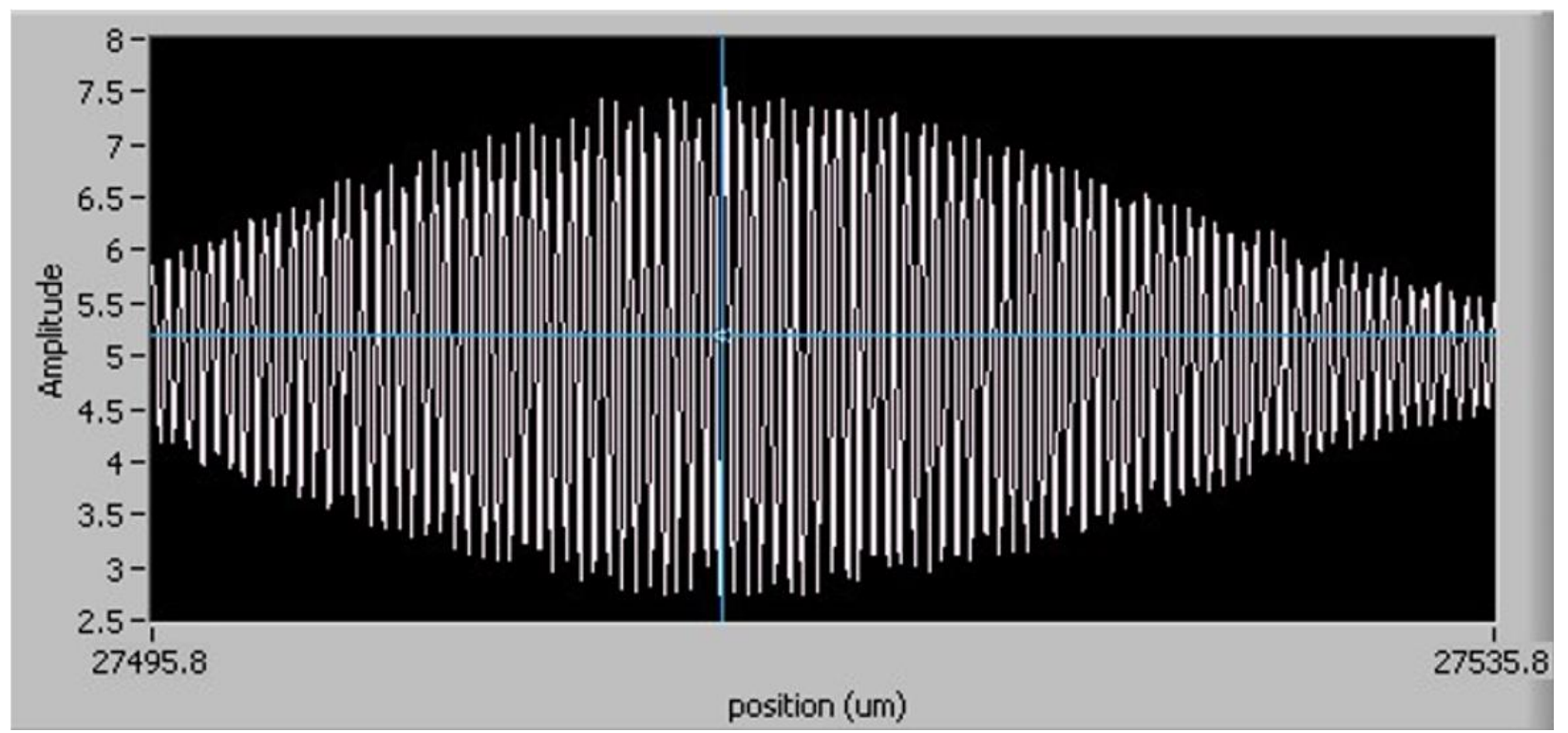

Figure 78 Finished autocorrelation with picked center 
5. Send A (stage S1T) to $122250 \mu \mathrm{m}$. Using interference from beams $A$ and $B$, repeat steps 2 through 4, scanning pulse B (stage S2T). Pulses A and B are now at zero time delay with respect to one another.

6. Using interference from beams $A$ and $C$, repeat steps 2 through 4, scanning Ref $+C$ (stage S1B), for the interdeck autocorrelation. Since stage S1B has double the optical path length of the previous scanned stages, set the scan speed to $.2 \mu \mathrm{m} / \mathrm{s}$ and the scan distance to 10 $\mu \mathrm{m}$ for equivalent parameters. All four beams are now at zero time delay with respect to one another. SHUTDOWN the replica focus program.

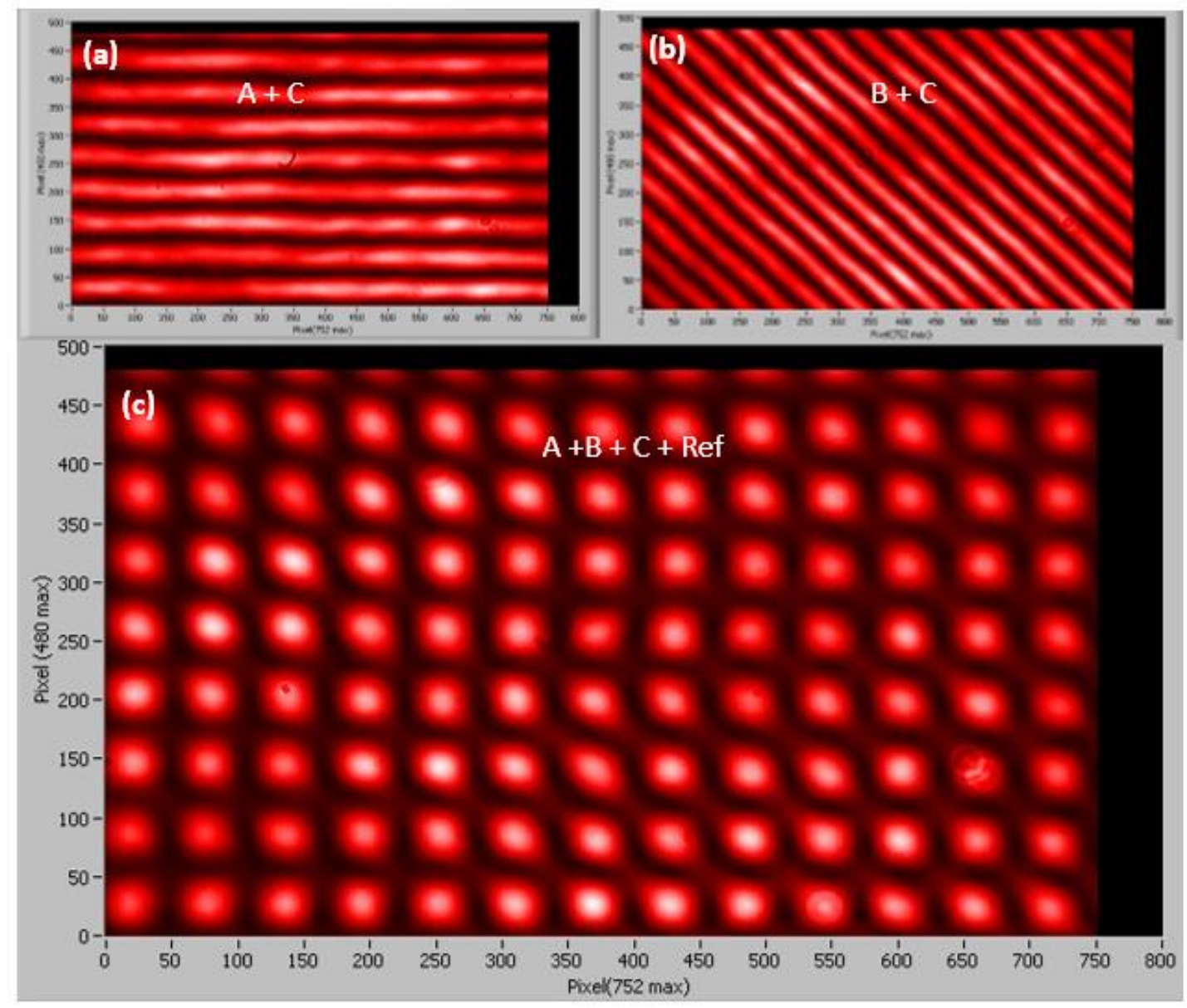

Figure 79 Interference between beams (a) A and C, (b) B and C, and (c) all four beams

7. After each set of beams has been zeroed, spatial overlap of all four beams can again be verified. Unblocking two diagonal beams gives diagonal interference, as in Figure 79 (a) Unblocking all beams should give the interference shown in Figure 79 (c). 
8. Add $7.5 \mu \mathrm{m}$ to the position determined for Ref $+\mathrm{C}$ stage (S1B) to set $\mathrm{T}=100 \mathrm{fs}$.

\section{A.6 Calibration of the SPEX 1702, 1 Meter Spectrometer}

1. Run CCD_FFT LabVIEW program and cool CCD to $-65 C$. This increases sensitivity and lowers the dark current. It takes less than 2 minutes. Set the exposure time to the minimum (1 millisecond). Electrical type noise should be seen on the output screen at about 670 counts. Below in Figure 80 you can see an example of what the CCD output looks like as it begins cooling and when it is finally cooled.

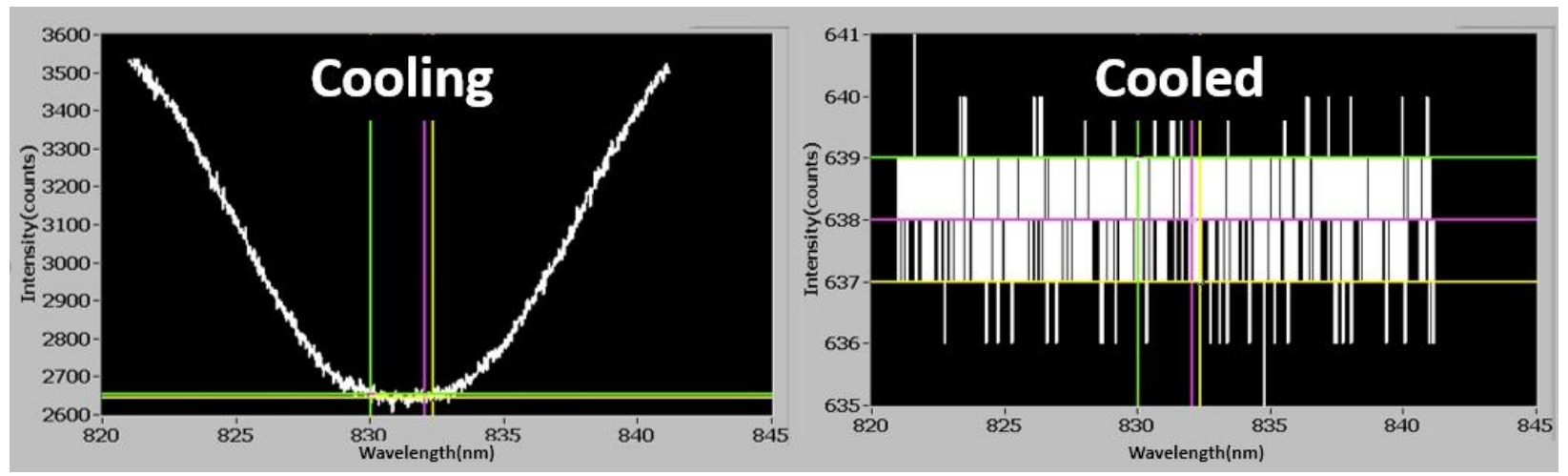

Figure 80 Cooling CCD

2. Turn on the calibration Krypton lamp and center in front of the spectrometer's input lens such that it does not saturate the detector. The detector will begin to saturate ate $58 \mathrm{k}$ counts, but if driven hard enough to reach $60 \mathrm{k}$. Therefore, $58 \mathrm{k}$ counts should be treated as the effective ceiling.

3. Observe the previous calibration, as it is often useful to monitor changes over any amount of time especially if the grating has not moved from the previous calibration. Set the wavelength offset to zero and slope to -1 . This output spectrum graph's horizontal axis is now pixels, increasing from left to right. 


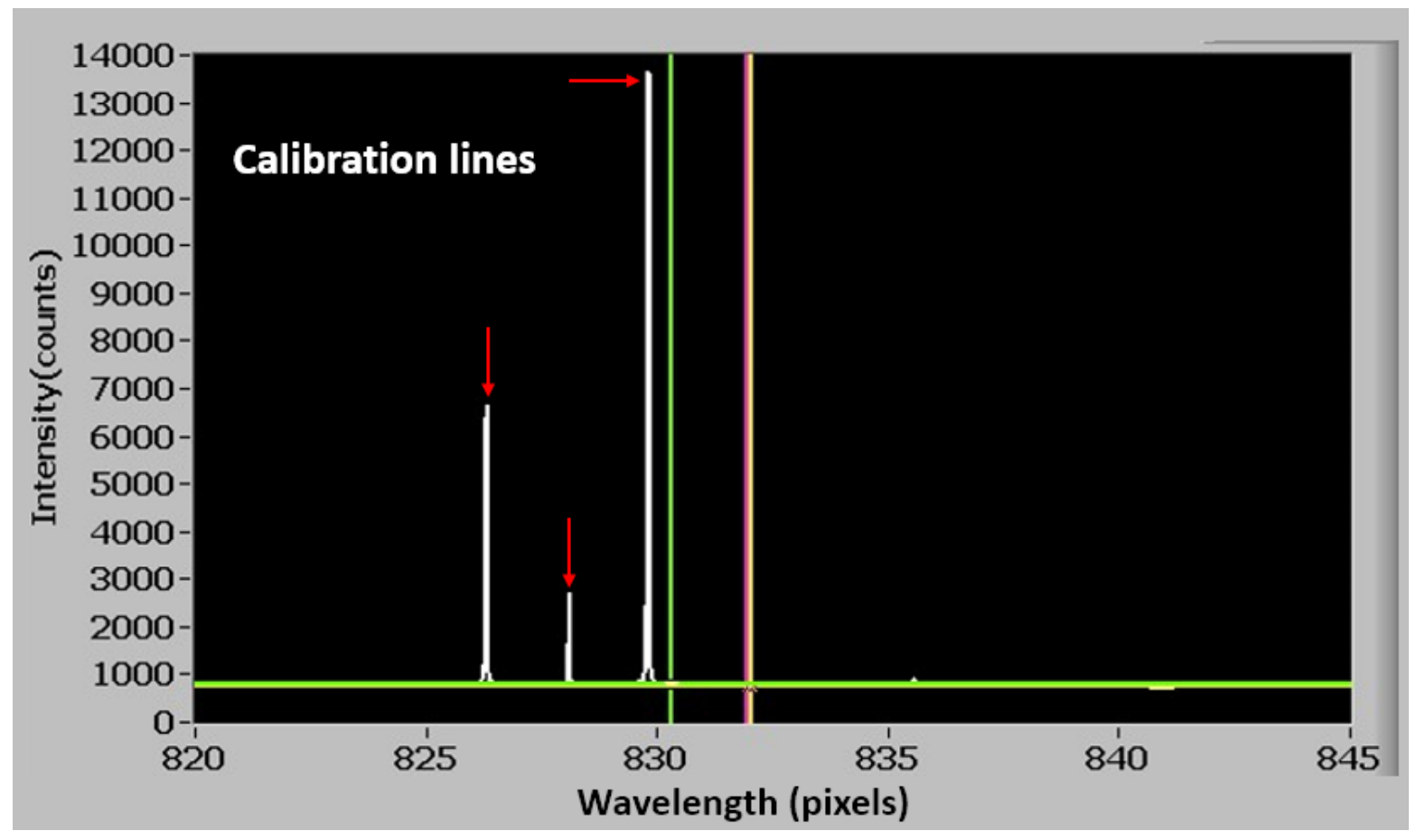

Figure 81 Calibration lines on spectrometer

4. Record the position of the lamp's peaks. Record a calibration spectrum as well for future reference.

5. Fitting: Using Origin (although many other programs would suffice) input the pixels into the $x$ column and their known spectral wavelength from the Pen Ray calibration lamp spectra into the $y$ axis and choose a linear fit. The fitting equation is $\lambda_{\text {output }}=m x_{\text {pixel }}+$ $\lambda_{\text {offset }}$, where $m$ is the slope in units of $\mathrm{nm} /$ pixel, $x_{\text {pixel }}$ is the center peak pixel on the detector, $\lambda_{\text {offset }}$ is the edge of the detector's spectral field of view and $\lambda_{\text {output }}$ is the final wavelength reported by the detector's calibrated output. All of the recorded peaks should fall along a nearly perfect line, if not, you have either erred the data entry or chosen the wrong known spectral wavelength to associate with the observed peaks.

a. While the offset will can greatly, the slope should be relatively constant because this is determined by the overall dispersion of the detector. Typical fluctuations for the current system are $\sim .0002 \mathrm{~nm} /$ pixel general. This error is associated with a possible one pixel error when choosing the center wavelength for the vary narrow peaks, typical of an elemental gas. 
6. Enter the computed slope and wavelength offset into the CCD_FFT program as well as the Master_Control Labview program.

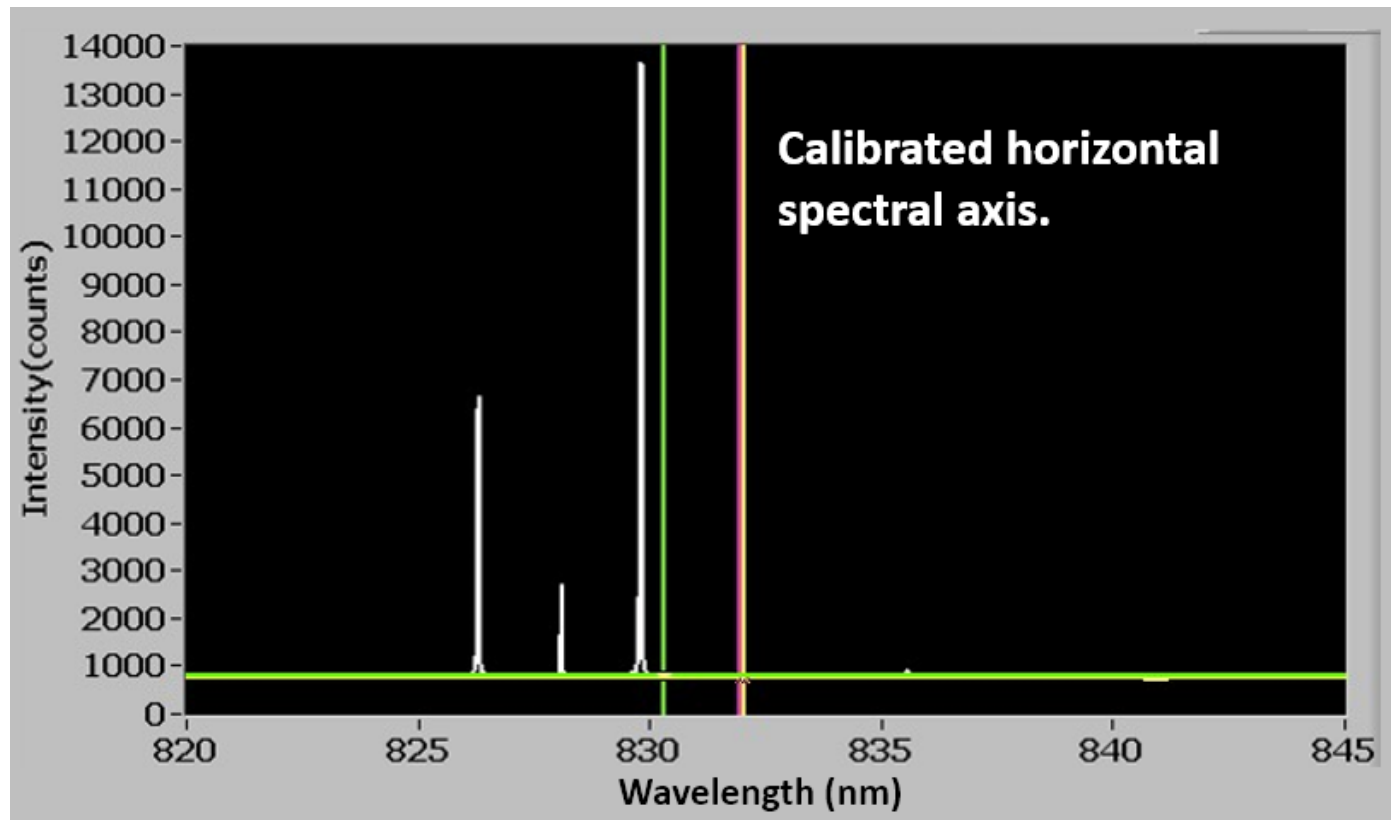

Figure 82 Calibrated Spectrometer

7. If you want to view different wavelengths, the grating must be manually rotated to the desired position by rotating the brass shaft on the side of the spectrometer and followed by recalibration. Other lamps may be necessary to have calibration peaks in the desired spectral field of view.

Note: Scanning is not possible (nor necessary) as there is currently no way to rotate the grating while maintaining continuous calibration. This could be implemented with a computer controlled motor.

\section{A.7 2D Data Acquisition Procedure, with Phase Retrieval}

1. Adjust the reference beam with ND filters so that it is $3-5 x$ the intensity of the signal.

2. Adjust the spectrometers ND filters so that the detector is not saturated but the signal still fills at least half of the working range.

3. Record incident and transmitted intensity on spectrometers ND filter using the spectrometer. This allows for accurate calculation of the optical density later. 
4. Set delay between the signal and ref using the reference retroreflector delay stage. The reference should arrive 5-10ps before the signal.

5. Examine ref and tracer spectral interferogram. Adjust so that it does not saturate the CCD. Use (several layers of) optical tissue in tracer beam to diffuse the light.

6. Record A, B, and C going through the sample individually. This will help with scatter estimation.

7. You also need to take a sapphire and background spectrum to calculate absorbance, if desired.

\section{Camera phase}

8. Place beamstop in front of sample. Unblock $\mathrm{C}$ and Ref/tracer

9. (Adjust camera exposure to see beams - you may need to do this for different decks.)

10. Record unlocked bottom-deck image with Mightex camera. Select horizontal slice and Filename: BottomUnlocked

11. Lock bottom deck.

12. Record locked bottom-deck image with Mightex camera. Select Filename: BottomLocked

13. Block $C$ and Ref/tracer (at MONSTR and Tracer). Remove beamstop from sample. Unblock $A$ and $\mathrm{B}$.

14. Record unlocked top-deck image with Mightex camera. Select Filename: TopUnlocked

15. Lock top deck.

16. Record locked top-deck image with Mightex camera. Select Filename: TopLocked

17. Unblock C

18. Lock interdeck.

Warning! MONSTR is now locked - all movements on table must be very gentle.

\section{Spectral interferograms}

19. Record signal-only spectrum. Select Filename: FWM

20. Block A and B. Unblock ref. (C can stay unblocked)

21. Record reference-only spectrum. [Use for processing both Spectral interferograms] Select Filename: $\boldsymbol{R E F}$ 
22. Unblock tracer and block reference.

23. Record tracer-only spectrum. Select Filename: tra

24. Unblock ref. Unblock $C$ if not already unblocked.

25. Lock the external loop. ( $C$ and ref MUST remain unblocked for the remainder of the time).

Warning! MONSTR and external loops are now locked - all movements on table must be very gentle.

26. Record ref+tracer spectral interferogram. Select Filename: traref

27. Block tracer and remove any optical tissue.

28. Unblock $A$ and B. (C and ref should be unblock already.)

29. Record signal+ref spectral interferogram. Select Filename: SI

30. Read phase-lock slopes form the loop filter switches and set those Booleans in the Master Control along with the type of scan, the number of steps, number of fringes (undersampling), and the stage speed.

31. Start the Scan.

\section{A.8 Processing Phase Retrieval Data}

1. Run prepfiles code as necessary during a scan to incorporate newly recorded data into the two-dimensional spectra

2. FTSA Matlab code:

Change ScanIndex-line 20- to desired scan and save copy of FTSAXX. Change WLMin and WLMax-line 32-to correct values from mono. Run the program.

PhiSI, PhiCam, PhiTR, and DelayTau0-lines 39 and 42-will be set using this procedure. TimeCut-line 34- will likely also be modified.

3. Observe Figure 4 (see Figure 83) and keep the scan running until the decay is finished. 
Figure 4 from FTSA

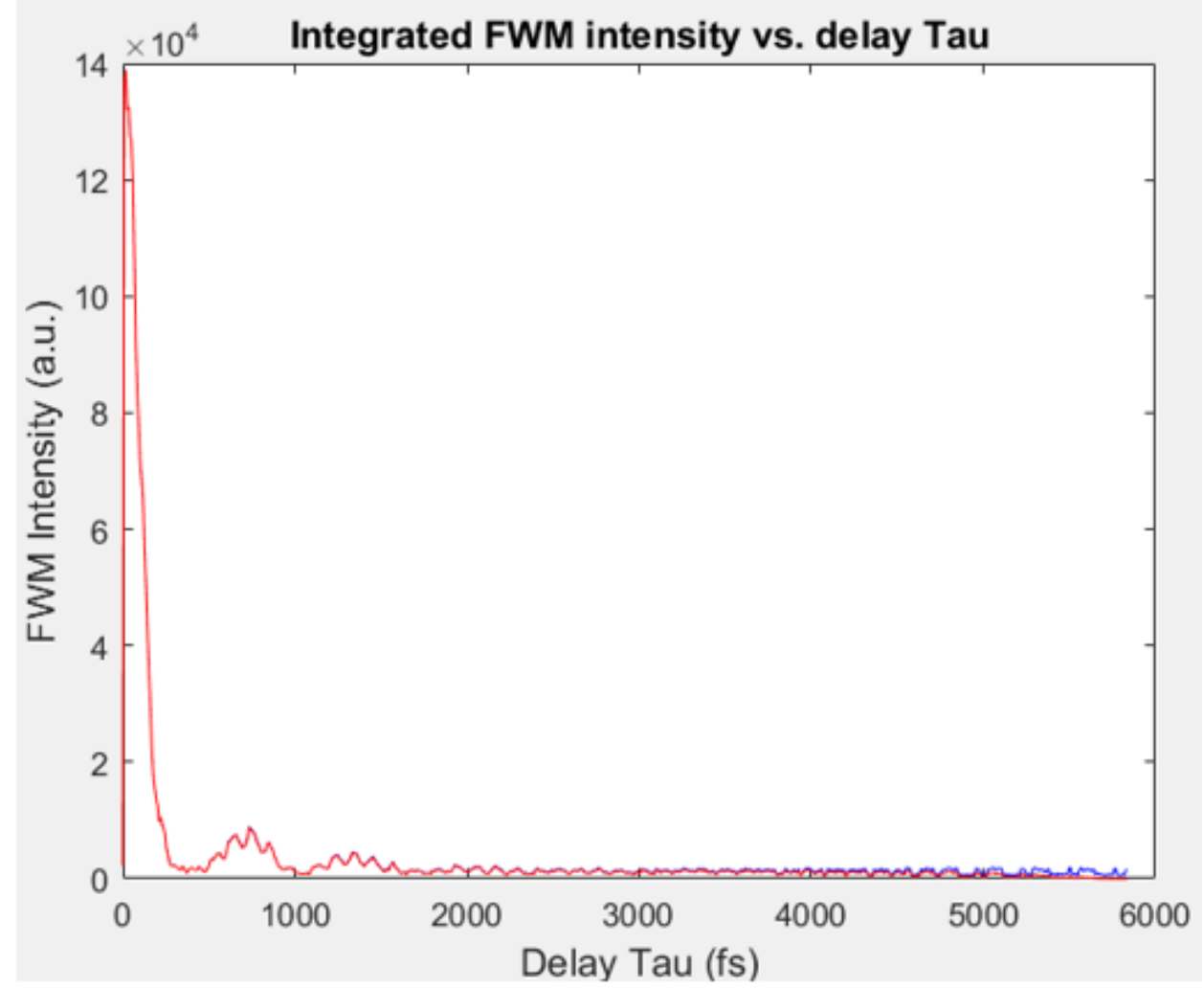

Figure 83 Figure 4 from FTSA showing the FWM decay as a function of the scanned time delay (tau in this case)

4. lineout (crunches replica focus traces)

a. Change FFindex to desired scan, then run it.

b. Copy and paste DPhiCam from Matlab command window to PhiCam in FTSA

5. LinAbsorption

Calculates absorbance and creates Phi_sum.dat for TracerSI. Phi_sum is phase vs energy. Only needs to be done once per valid absorbance spec

6. TracerSI

a. Change DataPath-line 6- to desired scan and save copy of TracerSIXX. Change WLMin and WLMax- line 7- to correct values from mono. Change FreqLowLmt and FreqHiLmt -line 11

b. Copy and paste DelayTau0 into DelayTau0 in FTSA 
c. Tweak TimeCut in FTSA as necessary by looking at Figure 22 (see Figure 84 below) and reading off a time shortly before signal.

Tracer SI Figure 22

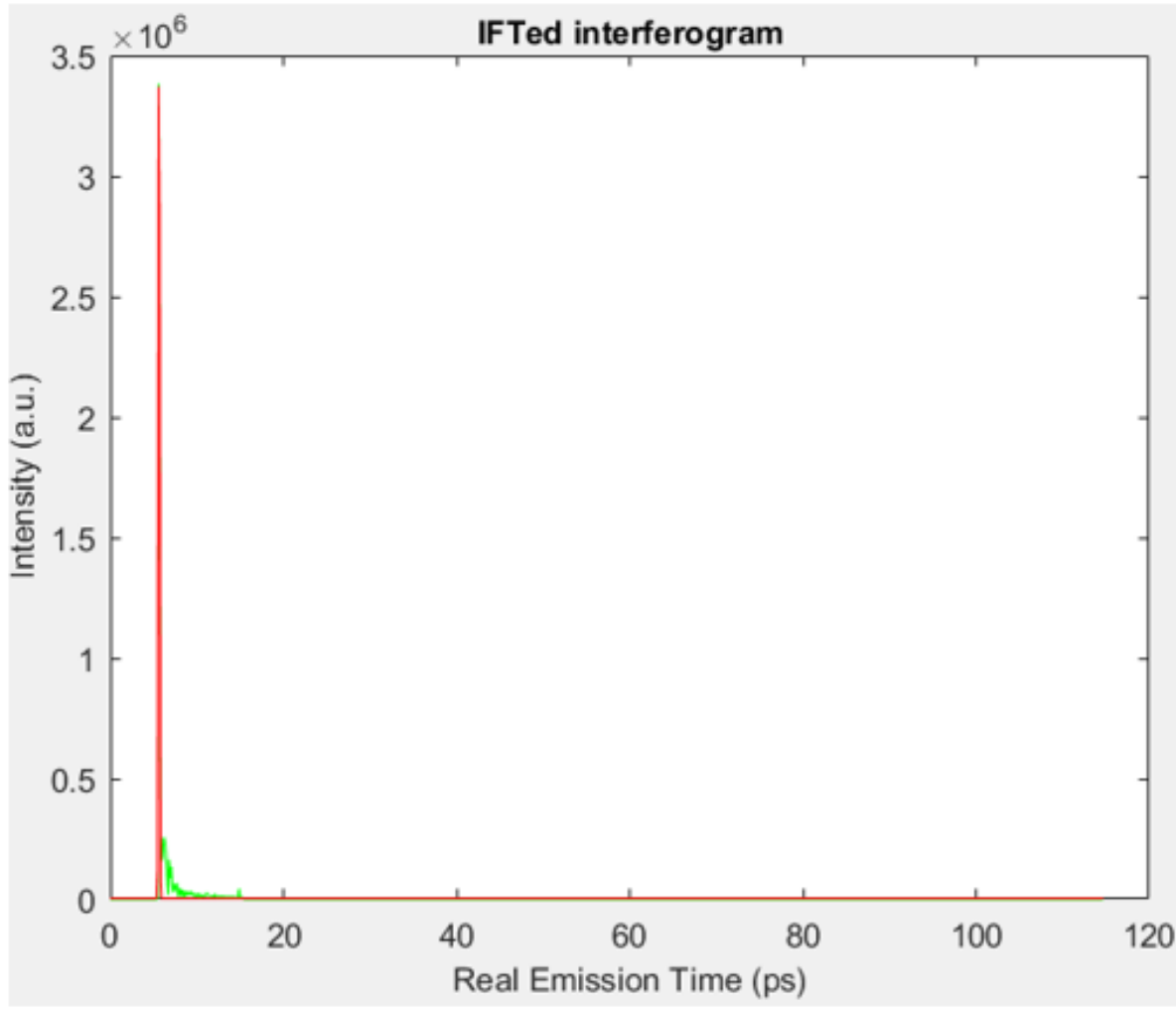

Figure 84 Figure 22 from TracerSI program showing the interferogram in the time domain and the extracted time delay between the reference and signal.

d. Using Figure 23 (see Figure 85 below), tweak PhiTR, phi2,Em0, and FrqShft on line 28 until the blue fit optimized. Finding the correct vertical offset (Ophi) is the goal.

e. Copy and paste PhiTR into PhiTR in FTSA 
Tracer SI Figure 23

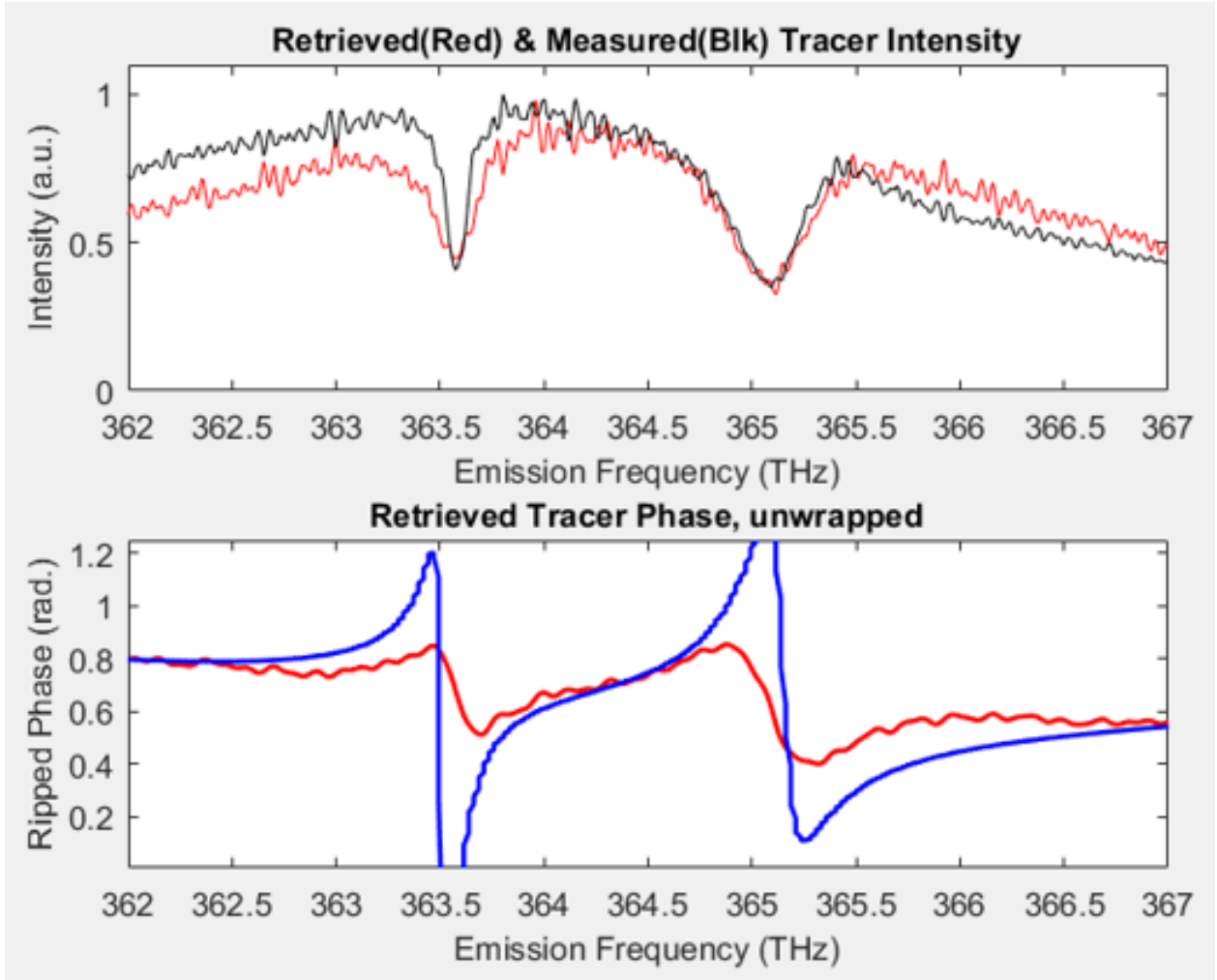

Figure 85 Figure 23 from TracerSI program showing the tracer intensity above and the unwrapped tracer phase below with the fit (blue) and observed (red) values

7. Run FTSA

a. From Figure 3 (see Figure 86 below) you need to pick the initial phase. This is usually somewhere to the left of the $\mathrm{HH}$ in GaAs, or whatever peak you have. Put this number into PhiSI-line 39 
Figure 3 from FTSA

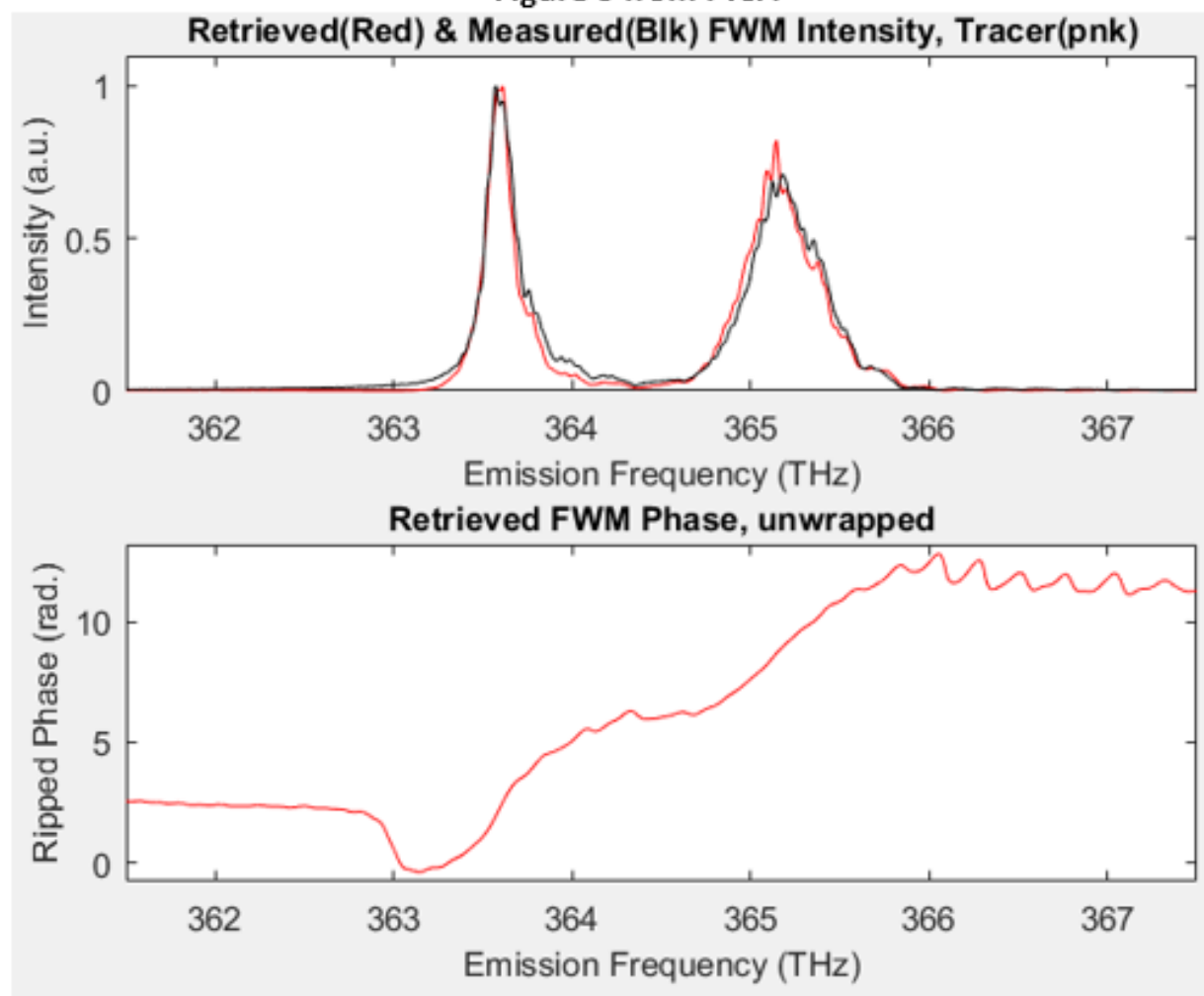

Figure 86 Figure 3 from FTSA program showing the FWM projections onto the emission axis above and the unwrapped FWM signal phase below.

b. Run FTSA "Whole"

c. Inspect Figure 9 (see Figure 87 below) to ascertain the quality of PhiSi and repeat a-c as necessary until optimized 
Figure 3 from FTSA

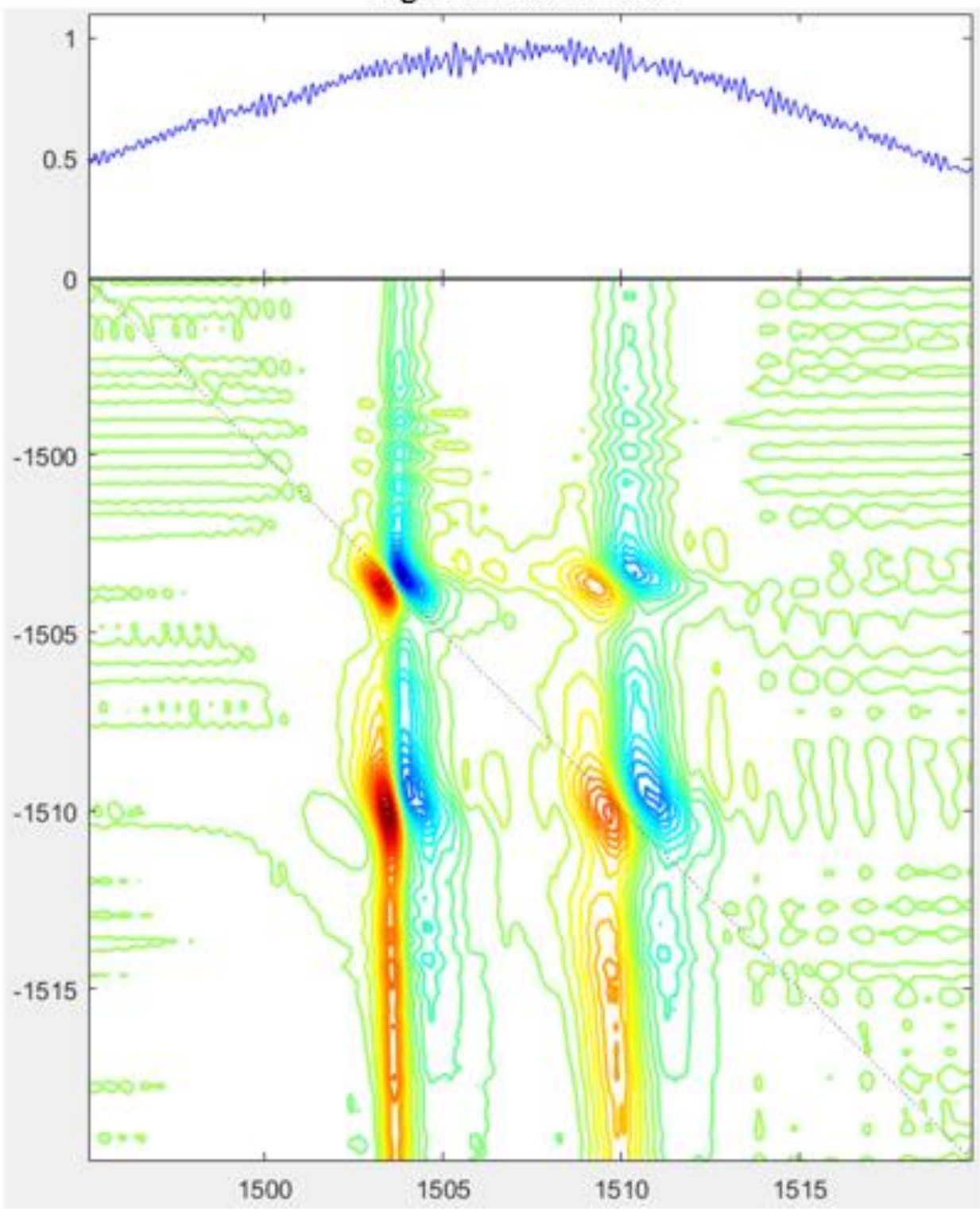

Figure 87 Figure 9 from FTSA program showing the real 2D spectrum and reference spectrum above

\section{A.9 Cryostation Purge Procedure}

This procedure should be done prior to each cooldown that is expected to last longer than one week in duration. It avoids the same mount from developing frost. When this does happen, warm up and perform the below procedure. Clean the sample faces and windows as well. This procedure is not in the manual. 
1. Attach nitrogen through the green port at the back of the control unit using $1 / 4$ " tubing. Nitrogen is only used beginning of the cool down, and then at the very end of the warm up when the system vents automatically to room pressure.

2. Set the regulator pressure to about $10 \mathrm{psi}$. Too high a pressure

3. To purge, once you're at room temperature go into manual mode and open the case valve (with vent valve closed). See Figure 88 for a diagram of the valve layout.

4. Turn the pump on, allow this to run until the pressure gets to 2 Torr (about 5 minutes or so).

5. Then close the case and turn off the pump (this order of operations keeps the pump safe)

6. Then open both the case and vent valve until atmospheric pressure (760 Torr) achieved (about 30 seconds).

7. Then repeat about twice more.

8. Then you may go into automatic cool down by just pressing cool down.

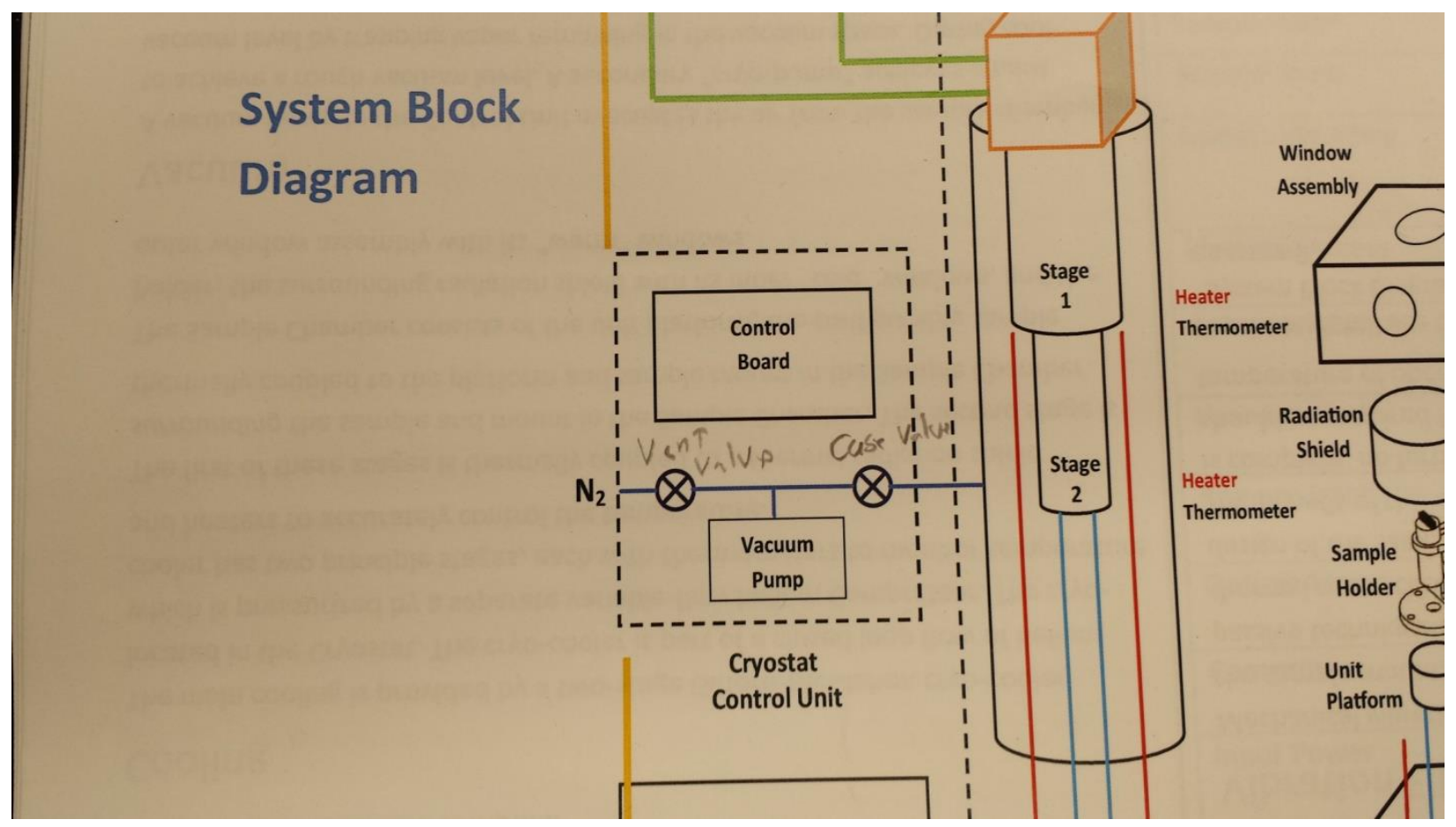

Figure 88 Cryostation diagram showing the valves used during the purge process.

\section{A.10 Prepare Spectra for FFT (Prep2DFiles.m)}

$\% \%$ Set directory for files and filenames 
clear all; clc;

\%Copy files over to dropbox?

Copy = 0;

$\mathbf{n}=\mathbf{2}$;

FFIndex = '64';

RootDir = strcat('.\2D',FFIndex,' ' ');

SpecDir = strcat(RootDir,'spectra\');

FileName = \{'FWM';'REF';'SI';'tra';'refa';'traref'\};

$\% \%$ Convert files recorded at starting position. Please notice the program

$\%$ will skip the missing files.

for $i=1: 6$

fid = fopen(strcat(RootDir,FileName\{i\},FFIndex,'.dat'),'r');

if (fid==-1), continue, end

[A,count] = fscanf(fid, ' $\% f \% f '$, [2 inf]);

fclose(fid);

$\%$ flip the matrix left to right if the calibration slope is positive.

$\% A=$ fliplr(A);

dlmwrite(strcat(RootDir,FileName\{i\},'00.dat'), $\left.\operatorname{round}\left(\mathbf{A}(2,:)^{\prime}\right)\right)$;

end

\%\% load all spectra into one matrix

while 1

$\%$ number to string

nStr = num2str(n); \% forward direction scanned

specfile = strcat(SpecDir,'spec',nStr,' .dat');

$\%$ open, read and close file

fid = fopen(specfile, 'r');

if ( $\mathrm{fid}==-1)$, break, end

\%FileInfo = dir(strcat('.|2D',FFIndex, '\spectra\spec',nStr, '.dat'))

Filelnfo = dir(specfile);

[Y, M, D, H, MN, S] = datevec(Filelnfo.datenum);

Time $(n, 1)=H^{*} 60 * 60+M N * 60+S$;

A = dlmread(specfile,' \t','B1..B1024');

fclose(fid);

$\%$ write to matrix

$\operatorname{M2D}(:, n)=\operatorname{round}(A)$;

$\%$ SpecTime $=(:, 1)=$ time;

$\% \quad M 2 D(:,(n+1) / 2)=\operatorname{round}(A)$;

$n=n+1$ 
$\% \quad n=n+2$

end

n;

$\% \%$ save matrix to file

$\%$ flip the matrix up side down if the calibration slope is positive.

$\%$ M2D=flipud(M2D);

dlmwrite(strcat('. I',RootDir, '\SIOtotal.dat'), M2D, '\t');

\% dlmwrite(strcat('.।',RootDir, '\SIOtotal.dat'), flipIr(M2D), '\t'); \%Backwards scan

dlmwrite(strcat('.।',RootDir,'\Time.dat'), Time, '\t');

clear A; clear M2D;

m= msgbox('All files for 2D program have been generated', 'Mission Completed');

if exist('m', 'var')

pause(.15)

delete(m);

clear('m');

end

\%\%\%\%\%\%\%\%\%\%\%\%\%\%\%\%\%\%\%\%\%\%\%\%\%\%\%\%\%\%\%\%\%\%\%\%\%\%\%\%\%\%\%\%\%\%\%\%\%\%\%\%\%

$\% \% \%$

if Copy $==1$

\%Copies files (including matlab) and directories to drop box, be sure to change the dates

clc

\%mkdir('D:\ADBgroupDocs\Dropbox\Defect Excitons 2014\Summer 2014\7-8-2014','2D09');

$\%$ Use this to make the directores up to 2D09

Date = '6-28-2016';

Fpath1 = strcat('D:\Data\Spring 2016\',Date);\%\% reference

Fpath2 = strcat('D:\ADBgroupDocs \Dropbox \Microcavities \',Date);\%destination

mkdir('D:\ADBgroupDocs \Dropbox \Microcavities\',Date);

\%Copies all .m files in the that day's directory over to Fpath2

copyfile(strcat(Fpath1, '\','*.m'),strcat(Fpath2,' ''),'f')

\%filenum = 1;

for $\mathbf{p}=$ str2num(FFIndex):str2num(FFIndex);

$\%$ if $\mathrm{n}<10 \%$ Folders less than 10 must have a ' 0 ' in front of the number..

$\% \quad \%$ Copies all the 2D0x\alloptical and output over

$\% \quad \% \%$ Makes 2Dxx directories greater than 10 up to $n$

\% mkdir(Fpath2,strcat('\2D0',num2str(n)));

$\%$

$\%$

copyfile(strcat(Fpath1,' \2D0',num2str(n),' 'alloptical'),...

strcat(Fpath2,'\2D0', num2str(n), '\alloptical'), 'f')

copyfile(strcat(Fpath1,'\2D0',num2str(n),'\Output'),... 


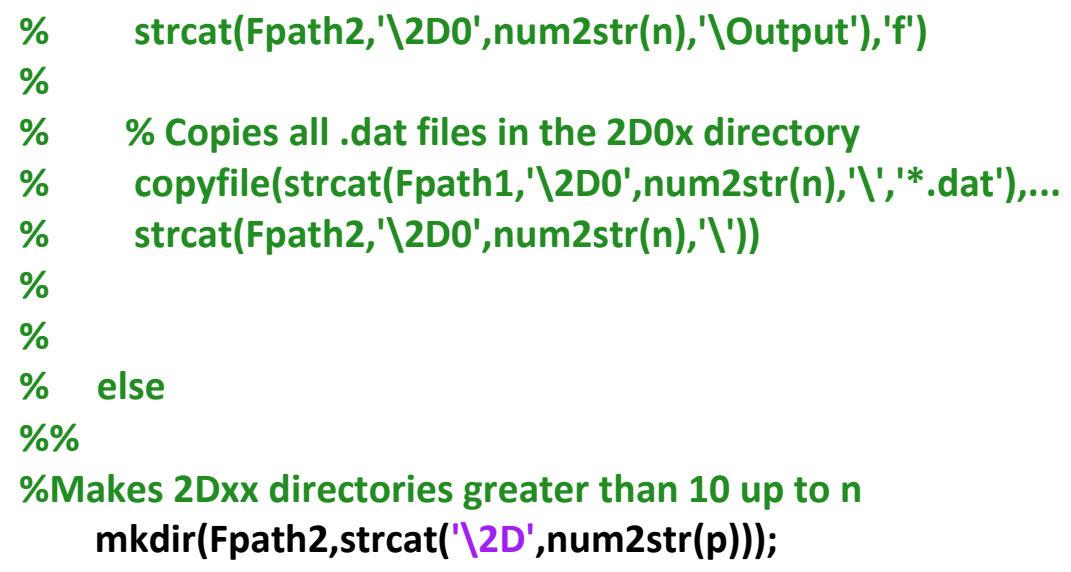

\section{A.11 FTSA (Fourier Transform Spectral Analysis) Code (FTSA.m)}

$\%$ reads intensity spectra of FWM, Reference and their spectral interferogram

\% to retrieve both amplitude and phase information of FWM, for S1 and S2

$\%$ scans.

format long g; clear all; clc; tic;

$\%$ HowProcessBtn = questdlg('Retrieve one spectrum or process whole data set?',...

$\% \quad$ 'Retrieve one or Process all', 'One', 'Whole', 'Cancel', 'Whole');

HowProcessBtn = 'Whole'; 
if strcmp(HowProcessBtn, 'Cancel') |isempty(HowProcessBtn) return; end;

\%------------------------------------------------------------------------

$\%$ constants and control parameters:

SpeedC $=2.99792458 \mathrm{E}+5 / 1.000345 ; \% \mathrm{~nm} / \mathrm{ps}$

$\%$ (already $1 / 2$ frequency value to correspond to 1/2 Bandwidth for Nyquist sampling)

HeNeWavelength=632.8;

FsHeNeFrg = SpeedC/HeNeWavelength;\%473.61338; \% HeNe(632.991nm) frequency THz

Frq2Egy $=4.135667516$; $\% 4.1356673$ conversion factor from $\mathrm{THz}$ to $\mathrm{meV}$

Abs_Fudge $=.2 /$ Frq2Egy;

$\%$ Frq2Egy = 1; $\quad$ \% use 1 if plot in $\mathrm{THz}$

\%length of the emission dimension (CCD length)

\%and the absorption dimension (zero padding included)

NEmiDim = 1024; NAbsDim = 2048;

\%Index number for 2D scans

ScanIndex = '1';

$\%$ The ratio of under-sampling, no under-sampling = 1

UdrSmplRatio = 4;

\% Determine whether this scan is rephasing (1) or non-rephasing (0)

isRephasing = 1;

\% Determine to do 2D-rephasing with SRDT spectrum (1) or not (0)

isSRDTrephasing = 0 ;

\% 2-axis phase cycling (0), otherwise (1).

isPhaseCycling = 1;

$\%$ Correct spectral phase for reflection geometry (1), otherwise (0)

isReflection = $\mathbf{0}$;

$\%$ Wavelengths of SPEX-750M spectrometer

WLMin =820.99199; WLMax =841.16555; \% nm

$\%$ settings used when plotting 2D spectra: Plot range and contour levels

FreqLowLmt = 358; FreqHiLmt $=363 ; \% \mathrm{THz}$

NContourLevels = 30;

$\%$ Cut off residual part near time zero after IFT FWM 4

TimeCut $=4 ; \quad \%$ ps

$\%$ Cut off part after signal

TimeCutAfter = 17; \%ps

\%Phaser factors for $\mathrm{SI}$, Camera and tracer

PhiSI = 0; PhiCam = -1.717068; PhiTR = .6; \%rad

$\%$ OPhiFct $=1.32$;

$\%$ delay between third pulse and reference

$\mathrm{PL}=0 ; \%$ Set to 1 if you have a PL spectrum to overlay

DelayTau0 = 8.8;

if isReflection

TimeCut_tr1 $=6.3$;

TimeCut_tr2 = 7.5; 


$$
\text { DelayTau_tr = 8.05; }
$$

end

\% Nyquis frequency, NO FACTOR OF 2, a full interferometer fringe is half fringe at OC NyquistFreq = FsHeNeFrg / UdrSmplRatio;

\% 2D matrix elments in the window defined by IndexLowLmt\&IndexHiLmt numbers or \%FreqLowLmt\&FreqHiLmt area plotted while those confined by NFirstCol\&NLastCol numbers saved

IndexLowLmt = round(((FreqLowLmt/SpeedC-1/WLMax)*NEmiDim)/(1/WLMin-1/WLMax)); IndexHiLmt = round(((FreqHiLmt/SpeedC-1/WLMax)*NEmiDim)/(1/WLMin-1/WLMax)); Multipier = 1.0;

NFirstCol = round(IndexLowLmt - Multipier*(IndexHiLmt-IndexLowLmt)/2.0);

NLastCol = round(IndexHiLmt + Multipier*(IndexHiLmt-IndexLowLmt)/2.0);

if (NFirstCol < 1) NFirstCol = 1; end

if (NLastCol > 1024) NLastCol = 1024; end

P2DLowEngyLmt = FreqLowLmt * Frq2Egy; P2DHiEngyLmt = FreqHiLmt * Frq2Egy;

\% Data Path and Filenames:

DataPath = strcat('.|2D',ScanIndex, '\');

Timefname = 'Time.dat';

FWMfname = 'FWM00.dat';

Slfname = 'SI00.dat';

Reffname = 'REF00.dat';

TIFWMfname = 'TI-FWM_tau.dat';

$\%$ Tracerfname='tra00.dat';

\% PLfname='PL00.dat';

if isReflection

$\%$ trafname = 'tra00.dat';

refafname = 'refa00.dat';

trareffname = 'traref00.dat';

end

\%NOTE: if using extension tube, comment out flipud(filename) operation!

$\%$ Also change this operation for matrix of all spectra (starting at line

$\%$ 214).

Time0 = dlmread(strcat(DataPath, Timefname), ' $\backslash \mathrm{t}$ ');

Time = Time0(:,1)-Time0(1,1);

FWMSpec1 = dlmread(strcat(DataPath, FWMfname), '\t');

FWMSpec1 = flipud(FWMSpec1); \% flipud(array) flips array up-down

SISpec = dlmread(strcat(DataPath, SIfname), '\t');

SISpec = flipud(SISpec);

RefSpec = dlmread(strcat(DataPath, Reffname), ' $\backslash t ')$;

RefSpec = flipud $($ RefSpec $)$; 


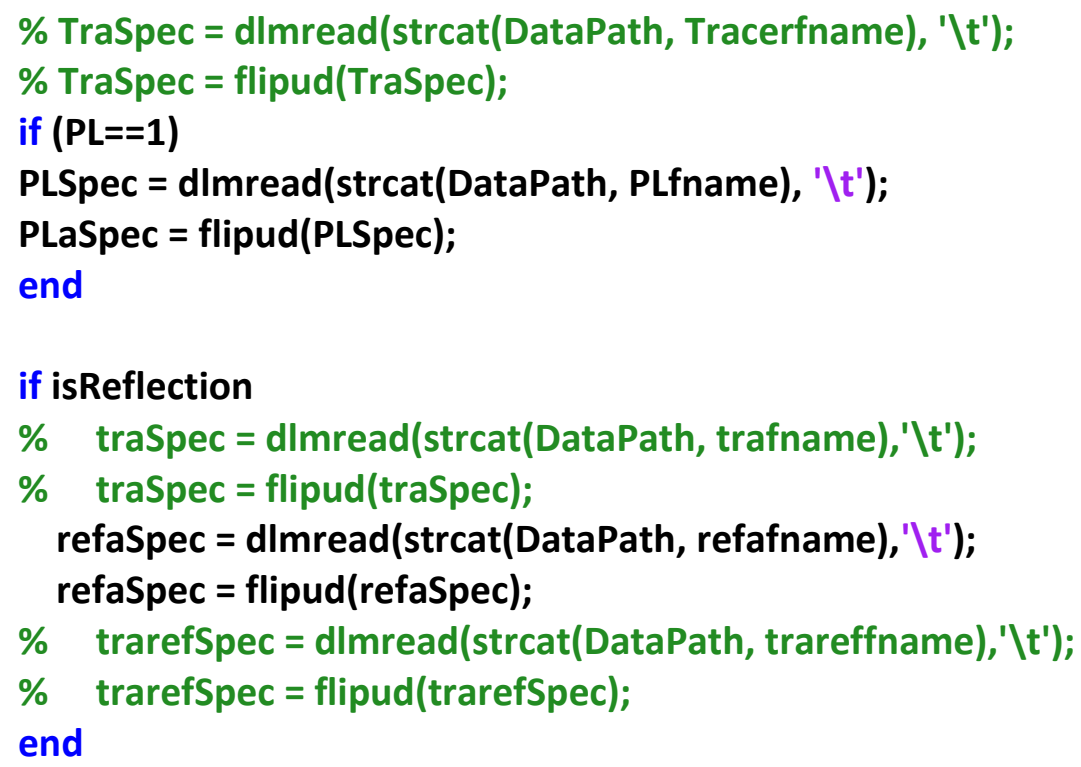


[PLSpec_THz, FreqMin, FreqMax] = resampleWL2Freq(PLSpec, WLMin, WLMax, NEmiDim); end

[InterfSpec_THz, FreqMin, FreqMax] = resampleWL2Freq(InterfSpec, WLMin, WLMax, NEmiDim);

if isReflection

\%trarefSpec $=$ trarefSpec - traSpec - RefRatio * refaSpec; \% already subtracted dark counts ave., see above

[refaSpec_THz, FreqMin, FreqMax] = resampleWL2Freq(refaSpec, WLMin, WLMax, NEmiDim);

[traSpec_THz, FreqMin, FreqMax] = resampleWL2Freq(traSpec, WLMin, WLMax, NEmiDim); $\%$ [trarefSpec_THz, FreqMin, FreqMax] = resampleWL2Freq(trarefSpec, WLMin, WLMax, NEmiDim);

end

$\%$ create frequency axis

FreqAxis = transpose $($ linspace $($ FreqMin, FreqMax, NEmiDim) $)$;

dlmwrite( strcat(OutDataPath, 'FWM_Ref_InterfSpec.dat'),...

[FreqAxis FWMSpec_THz RefSpec_THz InterfSpec_THz], '\t');

$\% \%$ Plot the spectra of FWM, Ref and Interferogram

fig1 = figure(1);

subplot(211); \% input spectra

plot(FreqAxis, RefSpec_THz, 'b-', FreqAxis, FWMSpec_THz*10.0, 'r-', FreqAxis, SISpec_THz, 'k');

AxisScale = axis; axis([ FreqLowLmt-1 FreqHiLmt+1 AxisScale(3:4) ]);

xlabel('Emission Frequency (THz)', 'FontSize', 10);

ylabel('Spectral Intensity (a.u.)', 'FontSize', 10);

title('Spectra of FWM(x10), Reference and their interferogram', 'FontSize', 10);

subplot(212); \% determined interference term

plot(FreqAxis, InterfSpec_THz, 'k-');

AxisScale = axis; axis([ FreqLowLmt-1 FreqHiLmt+1 AxisScale(3:4) ]);

xlabel('Emission Frequency (THz)', 'FontSize', 10);

ylabel('Spectral Intensity (a.u.)', 'FontSize', 10);

title('Interferometric Term', 'FontSize', 10);

saveas(gcf, strcat(OutDataPath, 'Fig1'), 'emf');

$\%$ Inverse Fourier transform to time domain (use FFT)

IFT_InterfSpec $=\mathbf{f f t}(\mathbf{f f t s h i f t}($ InterfSpec_THz, 1), NEmiDim, 1);

IFT_InterfSpec1 = IFT_InterfSpec;

$\% \%$

$\%$ Cut off residual part near time zero

NEmilnitCut =round(TimeCut*(FreqMax-FreqMin)); 
\% IFT_InterfSpec1(1 : NEmilnitCut) = 0;

$\%$ remove whole section from incorrect time ordering

\% IFT_InterfSpec1(NEmiDim/2+1: NEmiDim) = 0;\%Not sure about this

\%Remove part after signal

NEmilnitCut2 =round(TimeCutAfter*(FreqMax-FreqMin));

\% IFT_InterfSpec1(NEmilnitCut2 : 1024) = 0;

$\%$ Both times cuts done with error functions

IFT_InterfSpec2 = IFT_InterfSpec1;

pixel = 1:1:1024;

TrimWindow $=\left(\operatorname{erfc}\left(\left(\right.\right.\right.$ NEmilnitCut-pixel)/1) $/ 2{ }^{*} \operatorname{erfc}(($ pixel-NEmilnitCut2)/9)/2)';

IFT_InterfSpec1 = IFT_InterfSpec2. ${ }^{*}$ TrimWindow ;

$\%$ figure(70)

$\% \operatorname{plot}($ pixel,TrimWindow)

$\% \%$

$\%$ plot the time-domain information

fig2 = figure(2);

RTimeAxis = linspace( 0, (NEmiDim-1)/(FreqMax-FreqMin), NEmiDim ); plot(RTimeAxis, abs(IFT_InterfSpec), 'b-', RTimeAxis, abs(IFT_InterfSpec1), 'r-'); axis([ 0 RTimeAxis(end)/2 0 1.1*max(abs(IFT_InterfSpec1)) ]);

xlabel('Real Emission Time (ps)', 'FontSize', 10); ylabel('Intensity (a.u.)', 'FontSize', 10);

title('Fourier transformed interferogram', 'FontSize', 10);

saveas(gcf, strcat(OutDataPath, 'Fig2'), 'emf');

$\%$ Fourier transform back to spectral domain (use IFFT)

FT1 = ifft(IFT_InterfSpec1, NEmiDim, 1);

FT1Shift = ifftshift(FT1, 1);

$\%$ calculate retrieved signal

RetrFWM = FT1Shift./ sqrt(RefSpec_THz);

$\%$ find phase

PhaseRetrFWM = angle( RetrFWM .* exp(-i * 2*pi* FreqAxis .* DelayTau0) );

PhaseRetrFWM1 = unwrap(PhaseRetrFWM(NFirstCol:NLastCol));

$\%$ if isSRDTrephasing $\sim=0$

$\% \quad$ TrPhase $=$ TrPhase(NFirstCol:NLastCol);

$\%$ end

if isReflection

\% Inverse Fourier transform to time domain (use FFT)

IFT_trarefSpec $=\mathbf{f f t}(\mathrm{fftshift}($ trarefSpec_THz, 1), NEmiDim, 1);

IFT_trarefSpec1 = IFT_trarefSpec;

$\%$ Cut off residual part near time zero 


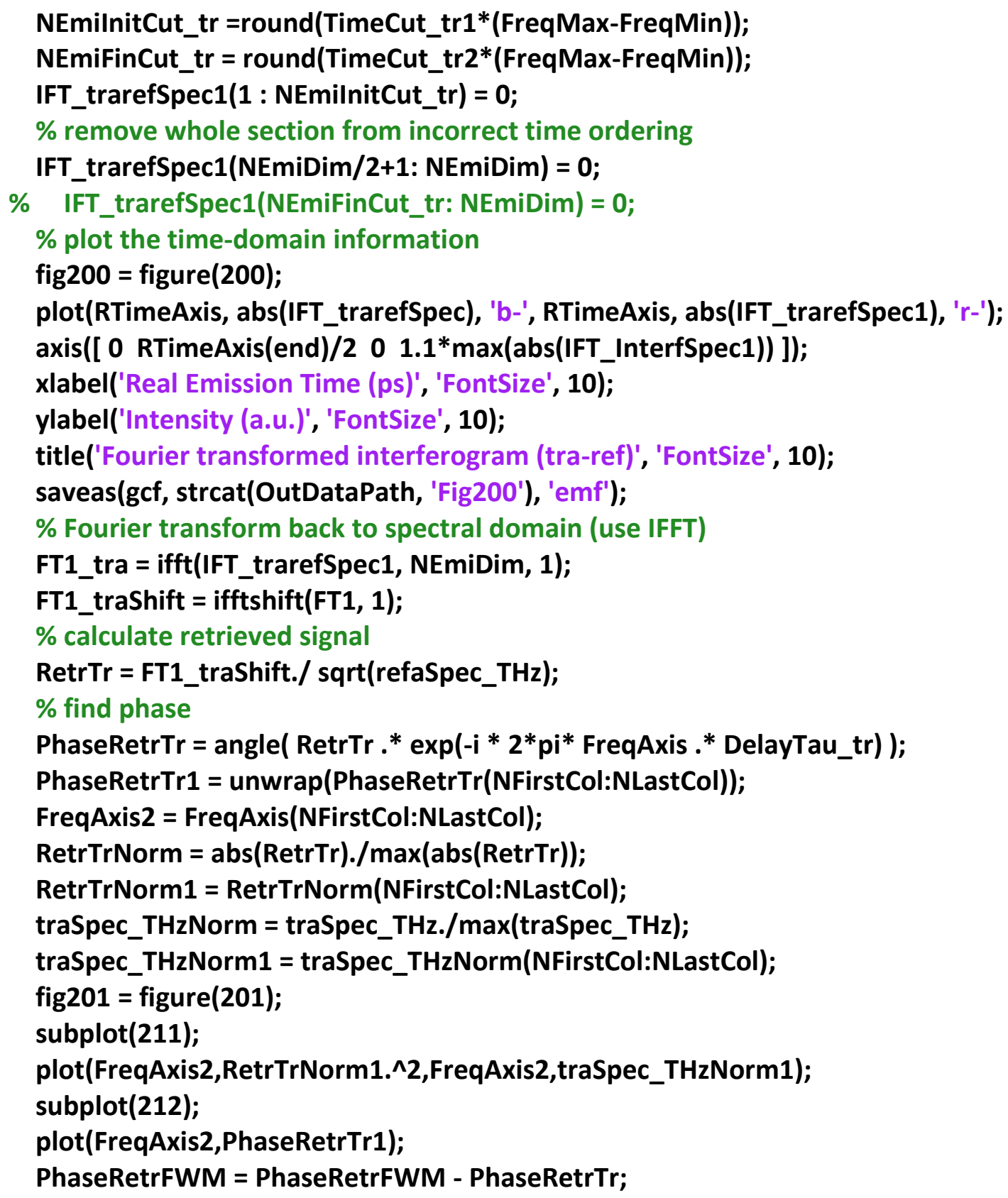


dlmwrite( strcat(OutDataPath, 'RetrEfield.dat'), [FreqAxis abs(RetrFWM) PhaseRetrFWM], '(t');

$\%$ Find the overall phase by matching Pump-probe measurement

if isSRDTrephasing == 0 \% no SRDTrephasing;

OPhiFct $=(\mathbf{P h i S I}-\mathrm{PhiCam}-\mathrm{PhiTR}) / \mathbf{p i} ; \quad \%$ varies in between 0 \& 2.0, in unit of pi else

SRDT0 $=$ sqrt(RefSpec_THz).$^{*}$ abs(RetrFWM).$^{*} \exp (\mathrm{i} *$ PhaseRetrFWM); \%TrSpec

$\% \quad$ SRDTO $=$ abs $($ RetrFWM $) . * \exp (i *$ PhaseRetrFWM $) ; \% \operatorname{TrSpec}$

for IdxM = $0: 1: 200$

SRDT1 = real( SRDT0 $*$ exp(-i* 2*pi * IdxM/200) );

SRDT1 = SRDT1 / (max(SRDT1)-min(SRDT1));

SRDTDevi(IdxM+1) = sqrt( sum(abs(SRDT1-DTSpec).^2, 1) /(length(SRDT1)-1) );

end

[MinDevi, MinDevilnd] = $\min ($ SRDTDevi);

OPhiFct $=2 *($ MinDevilnd-1)/200 \% in unit of pi

SRDTfn = real(SRDT0 * exp(-i* pi* OPhiFct));

SRDTfn = SRDTfn ./ (max(SRDTfn)-min(SRDTfn));

fig10 = figure(10);

plot( FreqAxis, SRDTfn, 'r-', FreqAxis, DTSpec, 'k.' );

axis([ FreqLowLmt-1 FreqHiLmt+1 $\min ($ SRDTfn)-0.2 $\max ($ SRDTfn)+0.2 ]);

xlabel('Emission Freq (THz)', 'FontSize', 12);

ylabel('Matched SRDT (normalized)', 'FontSize', 12);

\% legend('Pump-Probe with adjusted phase', 'Experimental SRDT', 'FontSize', 12);

title('Rephasing with SRDT', 'FontSize', 12);

text(FreqLowLmt-0.5, $\max ($ SRDTfn)+0.1, ...

strcat('Overall Phase: ', num2str(OPhiFct, '\%5.3f'), '*PI'), 'FontSize', 10);

text(FreqLowLmt-0.5, $\max ($ SRDTfn), ...

strcat('Std Dev.: ', num2str(MinDevi,'\%6.4f')), 'FontSize', 10);

saveas(gcf, strcat(OutDataPath, 'Fig10'), 'emf');

dlmwrite(strcat(OutDataPath, 'DTMatched.dat'), [FreqAxis DTSpec SRDTfn], '\t');

fig11 = figure(11);

plot( (0: 1 : 200)/200*2, SRDTDevi, '.');

xlabel('Overall phase factor (pi)', 'FontSize', 12);

ylabel('Weighted Standard Deviation', 'FontSize', 12);

end

RetrFWMR $=$ abs $($ RetrFWM $) . *$ real $\left(\exp \left(i^{*}\left(\right.\right.\right.$ PhaseRetrFWM-OPhiFct ${ }^{*}$ pi $\left.\left.)\right)\right)$;

RetrFWMI = abs(RetrFWM) .* imag( exp(i*(PhaseRetrFWM-OPhiFct*pi)) );

$\%$ save retrieved FWM efield, FreqAxis AMplitude, phase, Real and Imag

dlmwrite( strcat(OutDataPath, 'RetrFWMefieldAll.dat'), ...

[FreqAxis abs(RetrFWM) PhaseRetrFWM RetrFWMR RetrFWMI], '\t');

$\%$ save comparison, FreqAxis, Retred FWM intensity, phase and Expr FWM intensity 
dlmwrite( strcat(OutDataPath, 'CompRetrFWMefield_Expr.dat'),...

[ FreqAxis1 abs(RetrFWM1.^2)./max(abs(RetrFWM1.^2)) PhaseRetrFWM1 ...

FWMSpec_THz1./max(FWMSpec_THz1) ], '\t');

fig3 = figure(3);

subplot(211);

\% This is the factor used to normalize the 2D data

MaxSRFWMAmp $=\max (\operatorname{abs}($ RetrFWM1));

if $P L==1$

plot(FreqAxis1, abs(RetrFWM1.^2)./max(abs(RetrFWM1.^2)), 'r-', ...

FreqAxis1, FWMSpec_THz1./max(FWMSpec_THz1),'k-',...

FreqAxis1,TraSpec_Thz1./max(TraSpec_Thz1),'m-',...

FreqAxis1,PLSpec_Thz1./max(PLSpec_Thz1));

AxisScale = axis; $\quad$ axis([ FreqLowLmt FreqHiLmt 01.1 ]);

xlabel('Emission Frequency (THz)', 'FontSize', 10);

ylabel('Intensity (a.u.)', 'FontSize', 10);

title('Retrieved(Red) \& Measured(Blk) FWM Intensity, Tracer(pnk),PL (blu)', 'FontSize', 10);

else

plot(FreqAxis1, abs(RetrFWM1.^2)./max(abs(RetrFWM1.^2)), 'r-', ...

FreqAxis1, FWMSpec_THz1./max(FWMSpec_THz1),'k-');

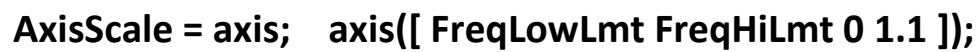

xlabel('Emission Frequency (THz)', 'FontSize', 10);

ylabel('Intensity (a.u.)', 'FontSize', 10);

title('Retrieved(Red) \& Measured(Blk) FWM Intensity, Tracer(pnk)', 'FontSize', 10);

end

subplot(212);

IndexLowLmt1 = round(IndexLowLmt - NFirstCol);

IndexHiLmt1 = round(IndexHiLmt - NFirstCol);

plot(FreqAxis1, PhaseRetrFWM1, 'r-');

axis([ FreqLowLmt FreqHiLmt...

min(PhaseRetrFWM1(IndexLowLmt1:IndexHiLmt1))-pi/8 ...

$\max ($ PhaseRetrFWM1(IndexLowLmt1:IndexHiLmt1))+pi/8]);

xlabel('Emission Frequency (THz)', 'FontSize', 10);

ylabel('Ripped Phase (rad.)', 'FontSize', 10);

title('Retrieved FWM Phase, unwrapped', 'FontSize', 10);

saveas(gcf, strcat(OutDataPath, 'Fig3'), 'emf');

$\% \%$ Process the data if confirmed at beginning

if strcmp(HowProcessBtn, 'One') return; end;

\% Retrieve FWM amplitude and phase at all delay tau

SIfname = strcat(DataPath, 'SIOtotal.dat');

MatIntf1 = dlmread(SIfname, ' $\backslash t ')$;

MatIntf1 = flipud(MatIntf1); \% See beginning for when to comment this out! 


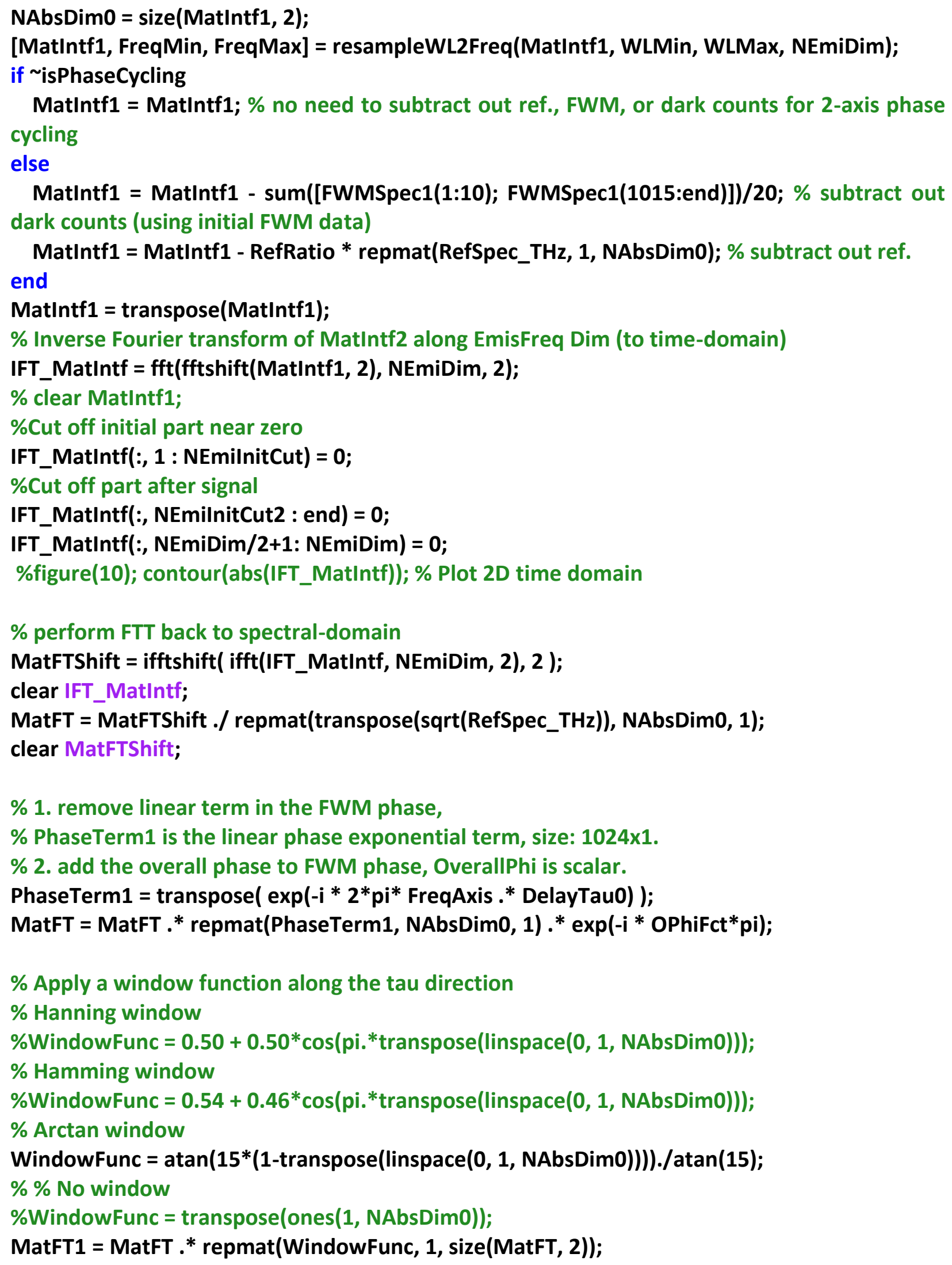


$\%$ Plot the retrieved FWM intensity vs. delay Tau at diff wavelength

fig4 = figure(4);

set(fig4, 'Name', 'Retrieved FWM intensity vs. Tau');

MatFTPrjInten = sum( (abs(MatFT)).^2, 2);

MatFT1PrjInten = sum( (abs(MatFT1)).^2, 2);

TauAxis = UdrSmplRatio * 1.0557134 * (0:1:(length(MatFTPrjInten)-1));

plot(TauAxis, MatFTPrjInten, 'b-', TauAxis, MatFT1PrjInten, 'r-');

xlabel('Delay Tau (fs)', 'FontSize', 12);

ylabel('FWM Intensity (a.u.)', 'FontSize', 12);

title('Integrated FWM intensity vs. delay Tau', 'FontSize', 12);

$\%$ write out retrieved TI-FWM to data file

dlmwrite(strcat(OutDataPath, 'RtrFWMvsTau.dat'), [ TauAxis' MatFTPrilnten ], '\t');

saveas(gcf, strcat(OutDataPath, 'Fig4'), 'emf');

$\% \% \%$ Diagnostic plot with real time axis

$\%$ fig12 = figure(12);

\% set(fig4, 'Name', 'Retrieved FWM intensity vs. Tau');

\% plot(Time, MatFTPrjInten, 'b-', Time, MatFT1PrjInten, 'r-');

\% xlabel('Time after first Spec(seconds)', 'FontSize', 12);

$\%$ ylabel('FWM Intensity (a.u.)', 'FontSize', 12);

$\%$ title('Integrated FWM intensity vs. delay Tau', 'FontSize', 12);

\% dlmwrite(strcat(OutDataPath, 'RtrFWMvsRealTime.dat'), [ Time MatFTPrjInten ], '\t');

$\% \%$ FFT stuff of time domain signal

$\% \mathrm{t}(:, 1)=0: 1: \max ($ Time); $\% 1 \mathrm{~s}$ increments from 0 to end of Time

$\% \% \%$ Interpolate the data

$\%$

$\% v q=$ interp1(Time,MatFTPrjInten,t,'spline');

$\%$

$\%$

$\%$ \% figure(13) \%show interpolated data

$\% \% \operatorname{plot}(t, v q, '-g ') ;$

$\% \mathrm{~L}=$ =length(Time);

$\%$

$\%$ NFFT $=2^{\wedge}$ nextpow2 $(2 * \mathrm{~L}) ; \%$ Next power of 2 from length of $y$

$\%$

$\%$ FFT_FWM $=\mathrm{fft}(\mathrm{vq}(:, 1), \mathrm{NFFT})$;

$\%$ figure(14)

$\% f=1 / 2 *$ linspace $(0,1, N F F T / 2+1)$;

$\% \operatorname{plot}\left(f, 1 * a b s\left(F F T \_F W M(1: N F F T / 2+1)\right)\right)$

$\%$ xlabel('Frequency(hz)');

$\%$ ylabel('Amplitude');

$\%$ title('FFT of FWM vs tau');

\% saveas(gcf, strcat(OutDataPath, 'Fig13FFT'), 'emf');

\% dlmwrite(strcat(OutDataPath, 'FFT_FWMvsRealTime.dat'), [ Time MatFTPrjInten ], '\t'); 
clear MatFTPrjInten MatFT1PrjInten;

$\% \%$

$\%$ Pad zeros at the end of matrix to increase length in tau

\% F.T. FWM matrix with respect to tau (along the absorption dimension / row)

MatFTPadded = [ MatFT1; zeros(NAbsDim-size(MatFT1,1), $\operatorname{size}($ MatFT1,2)) ];

M2D = fftshift(fft((MatFTPadded), NAbsDim, 1 ), 1);

$\% \mathrm{M} 2 \mathrm{D}=\mathrm{fft}(($ MatFTPadded $)$, NAbsDim, 1$)$;

$\%$ clear MatFT MatFT1 MatFTPadded;

\% -------------------------------------------------------------------------

$\%$ Generate spectrum in the right range from the undersampled data

if (UdrSmplRatio == 64) ||(UdrSmplRatio == 128) ||(UdrSmplRatio == 4) ||(UdrSmplRatio ==

8) | | (UdrSmpIRatio == 16) | | (UdrSmplRatio == 32) | | (UdrSmplRatio == 1)

EmiFreqStep $=($ FreqMax - FreqMin $) /$ NEmiDim;

AbsFreqStep $=2 *$ NyquistFreq/NAbsDim;

NSmplDim = round((NLastCol-NFirstCol)*EmiFreqStep/AbsFreqStep);

if isRephasing == $1 \%$ Rephasing measurement

$\%$ Freq observed $=$ Original Freq $-2 * N *$ NyquistFreq

$\%$ Take only the half side with negative absor frequencyfor

for $k=1$ :NSmplDim

FreqUnfolded $=$ FreqMin + NFirstCol*EmiFreqStep $+(\mathbf{k}-1) *$ AbsFreqStep;

NFolding = round(FreqUnfolded/(2*NyquistFreq));

FreqFolded $=$ FreqUnfolded $-2 *$ NFolding*NyquistFreq;

NAbsRow = round((FreqFolded + NyquistFreq)/AbsFreqStep);

M2DUdISmpl(k,:) = M2D(NAbsRow, NFirstCol:NLastCol );

AbsFreqAxis $(k)=$ - FreqUnfolded;

end

M2DUdlSmpl=flipud(M2DUdlSmpl);

AbsFreqAxis =flipud(transpose(AbsFreqAxis))+Abs_Fudge;

else \% Non-Rephasing measurement

$\%$ Freq observed $=2 * N *$ NyquistFreq - Original Freq

$\%$ Take only the second half part with positive absor frequency

for $k=1$ :NSmplDim

FreqUnfolded = FreqMin + NFirstCol*EmiFreqStep + (k-1)*AbsFreqStep;

NFolding = round(FreqUnfolded/(2*NyquistFreq));

FreqFolded $=2 *$ NFolding $*$ NyquistFreq - FreqUnfolded;

NAbsRow = round((FreqFolded + NyquistFreq)/AbsFreqStep);

M2DUdlSmpl(k,:) = M2D(NAbsRow, NFirstCol:NLastCol );

AbsFreqAxis(k) = FreqUnfolded;

end

AbsFreqAxis =transpose(AbsFreqAxis)+Abs_Fudge;

end 


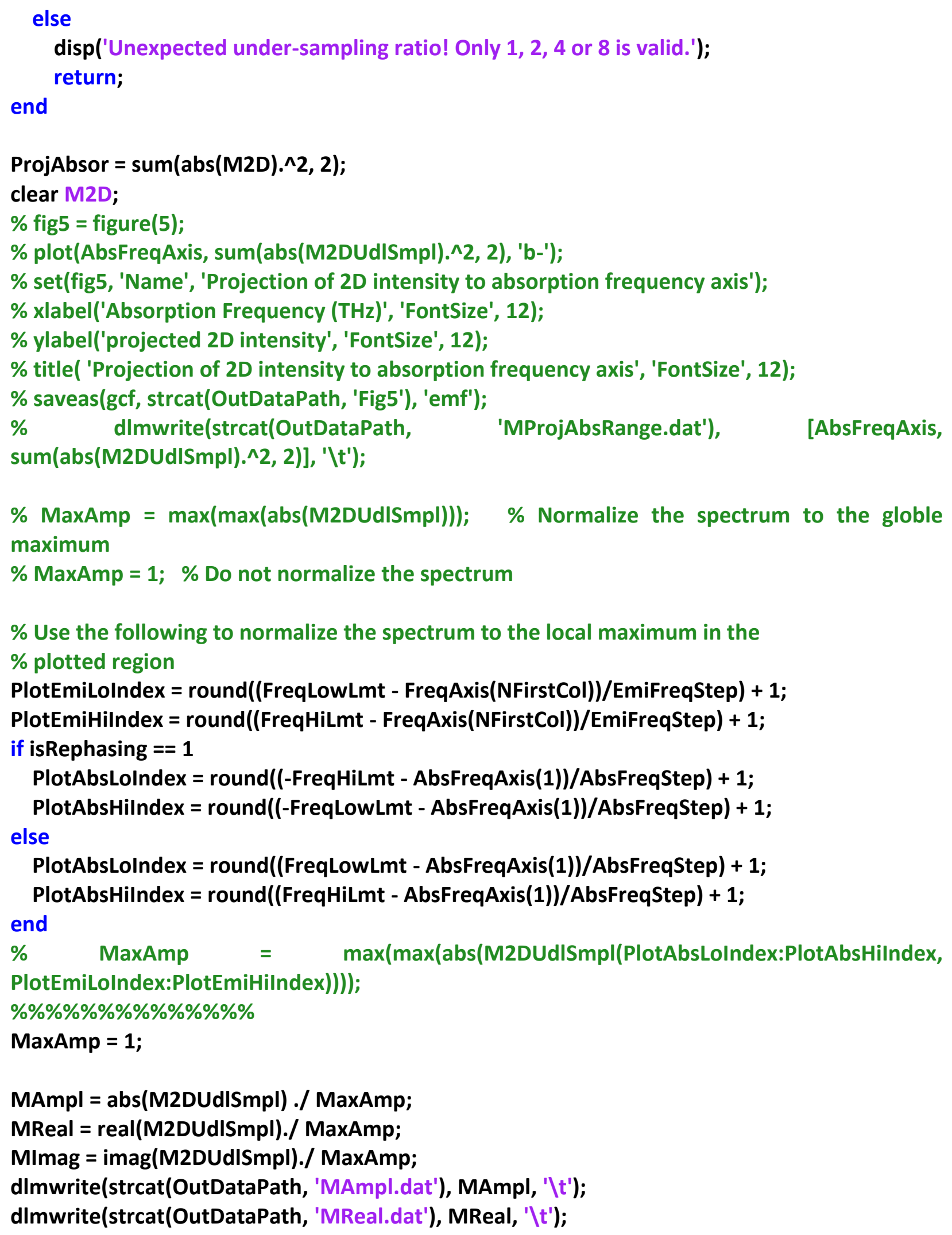


dlmwrite(strcat(OutDataPath, 'Mlmag.dat'), MImag, '\t');

VMax $=\max (\max (M A m p l)) ;$

$\operatorname{MDim}(1: 2)=[$ FreqAxis(NFirstCol), FreqAxis(NLastCol)];

$\operatorname{MDim}(3: 4)=[$ AbsFreqAxis(1), AbsFreqAxis(end)];

$\%$ Export the frequency ranges of 2D matrix to a file, in the order of

\% EmiFreq_min, EmiFreq_max, AbsFreq_min, and AbsFreq_max.

fid = fopen( strcat(OutDataPath,'MFreqRange.txt'), 'w');

fprintf(fid, 'NFirstCol \t NLastCol \t NSmplDim \r|n');

fprintf(fid, '\%10.0f $\backslash t$ \%9.0f $\backslash t$ \%10.0f $\backslash r \backslash n '$ ', NFirstCol,NLastCol,NSmplDim);

fprintf(fid, 'EmiFreq_min \t EmiFreq_max \t AbsFreq_min \t AbsFreq_max \r\n');

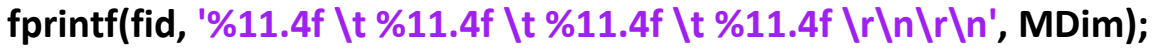

MDimEngy = Frq2Egy. ${ }^{*}$ MDim;

fprintf(fid, 'EmiEngy_min \t EmiEngy_max \t AbsEngy_min \t AbsEngy_max \r\n');

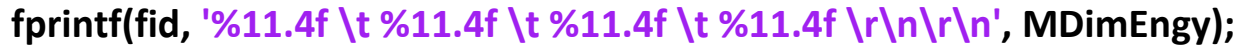

$\%$ Output important paprameters to the file

fprintf(fid, 'TimeCut = \%5.3f $\backslash r \backslash n$ ', TimeCut);

fprintf(fid, 'OverallPhiFactor $=\% 5.3 f \backslash r \backslash n '$ ', OPhiFct);

fprintf(fid, 'PhiSI = \%5.3f $\backslash r \backslash n$ ', PhiSI);

fprintf(fid, 'PhiCam = \%5.3f \r|n', PhiCam);

fprintf(fid, 'PhiTR = \%5.3f \r\n', PhiTR);

fprintf(fid, 'DelayTau0 = \%5.3f $\backslash r \backslash n$ ', DelayTau0);

fprintf(fid, 'DataPath = \%s \r|n', DataPath);

fprintf(fid, 'Rephasing? = \%g \r\n', isRephasing);

fprintf(fid, 'Peak of SR-FWM ampl at tau=0: \%7.4g \r\n', MaxSRFWMAmp);

fprintf(fid, 'Peak of 2D amplitude matrix: \%7.4g \r\n', MaxAmp);

fprintf(fid, 'Normalization factor: \%7.4g \r\n', MaxAmp/MaxSRFWMAmp);

fclose(fid);

$\%$ Plot Amp, real and imaginary parts of 2D data

[gEmiFreq, gAbsFreq] = meshgrid(linspace( MDimEngy(1), MDimEngy(2), NLastCol-NFirstCol+1 ),...

linspace( MDimEngy(3), MDimEngy(4), NSmplDim));

if isRephasing $==1 \%$ Rephasing scan

FigName = strcat('Rephasing data of: ', DataPath);

AxisRange $=[$ P2DLowEngyLmt P2DHiEngyLmt -P2DHiEngyLmt -P2DLowEngyLmt ];

DiagnlLine = [linspace(P2DLowEngyLmt, P2DHiEngyLmt, 20); linspace(-P2DLowEngyLmt, P2DHiEngyLmt, 20)];

elseif isRephasing $=\mathbf{0} \%$ Non-Rephasing scan

FigName = strcat('Non-Rephasing data of: ', DataPath);

AxisRange = [ P2DLowEngyLmt P2DHiEngyLmt P2DLowEngyLmt P2DHiEngyLmt ];

DiagnlLine = [linspace(P2DLowEngyLmt, P2DHiEngyLmt, 20); linspace(P2DLowEngyLmt, P2DHiEngyLmt, 20)]; 
end

\%opengl neverselect;

$\% \% \%$ linear absorption data

$\%$ absdata = dlmread(strcat('.\','absorbanceB.dat'), '\t');

$\%$ linabs = absdata(:,2);

$\%$ linenergy = absdata(:,1);

$\%$ \% linenergy = resampleWL2Freq(linlambda, WLMin, WLMax, NEmiDim)*Frq2Egy;

specenergy = FreqAxis*Frq2Egy;

\% AxisRange1 = [ P2DLowEngyLmt P2DHiEngyLmt 0 1.1*max(linabs) ];

AxisRange1 = [ P2DLowEngyLmt P2DHiEngyLmt 0 1.1 ];

$\% \%$

\section{Figure 7}

Plot Amp, real and imaginary parts of 2D data

[gEmiFreq, gAbsFreq] = meshgrid(linspace( MDimEngy(1), MDimEngy(2), NLastCol-NFirstCol+1 ),...

linspace( MDimEngy(3), MDimEngy(4), NSmpIDim));

FigName = strcat('Rephasing data of: ', DataPath);

opengl neverselect;

fig7 = figure(7);

set(fig7, 'Position', [ 100501000500 ]);

set(fig7, 'Name', FigName);

subplot(131);

hFig = contour(gEmiFreq, gAbsFreq, MAmpl, linspace(0, VMax, NContourLevels), 'LineWidth', 1.5);

line(DiagnILine(1,:), DiagnILine(2,:), 'LineStyle', ':', 'LineWidth',1, 'Color', [0 0 0]);

line(D1Line(1,:), D1Line(2,:), 'LineStyle', ':', 'LineWidth',1, 'Color', [0 0 0]);

line(D2Line(1,:), D2Line(2,:), 'LineStyle', ':', 'LineWidth',1, 'Color', [0 0 0 $]$ ]);

line(D1LineH(1,:), D1LineH(2,:), 'LineStyle', ':', 'LineWidth',1, 'Color', [ $\left.\begin{array}{ll}0 & 0\end{array}\right]$ );

line(D2LineH(1,:), D2LineH(2,:), 'LineStyle', ':', 'LineWidth',1, 'Color', [0 0 0]);

line(D12LineH(1,:), D12LineH(2,:), 'LineStyle', ':', 'LineWidth',1, 'Color', [ $\left.\left.\begin{array}{lll}0 & 0 & 0\end{array}\right]\right) ;$

axis( AxisRange ); colormap jet;

ylabel('Absorption Energy (meV)')

title('Amplitude');

subplot(132);

$\operatorname{MReal}($ end, end-1)= -1; MReal(end, end)=1;

hFig = contour(gEmiFreq, gAbsFreq, MReal, linspace(-VMax, VMax, NContourLevels), 'LineWidth', 1.5);

line(DiagnILine(1,:), DiagnlLine(2,:), 'LineStyle', ':', 'LineWidth',1, 'Color', [0 0 0]);

line(D1Line(1,:), D1Line(2,:), 'LineStyle', ':', 'LineWidth',1, 'Color', [ $\left.\begin{array}{lll}0 & 0 & 0\end{array}\right]$ );

line(D2Line(1,:), D2Line(2,:), 'LineStyle', ':', 'LineWidth',1, 'Color', [ $\left.\left.\begin{array}{lll}0 & 0 & 0\end{array}\right]\right) ;$

line(D1LineH(1,:), D1LineH(2,:), 'LineStyle', ':', 'LineWidth',1, 'Color', [0 0 0]);

line(D2LineH(1,:), D2LineH(2,:), 'LineStyle', ':', 'LineWidth',1, 'Color', [0 0 0]);

line(D12LineH(1,:), D12LineH(2,:), 'LineStyle', ':', 'LineWidth',1, 'Color', [ $\left.\begin{array}{lll}0 & 0 & 0\end{array}\right]$ ); 
axis( AxisRange ); colormap jet;

xlabel('Emission Photon Energy (meV)')

title('Real');

subplot(133);

MImag(end, end-1)= -1; MImag(end, end)= 1;

hFig = contour(gEmiFreq, gAbsFreq, MImag, linspace(-VMax, VMax, NContourLevels), 'LineWidth', 1.5);

line(DiagnILine(1,:), DiagnILine(2,:), 'LineStyle', ':', 'LineWidth',1,'Color', [0 0 0]);

line(D1Line(1,:), D1Line(2,:), 'LineStyle', ':', 'LineWidth',1, 'Color', [0 0 0]);

line(D2Line(1,:), D2Line(2,:), 'LineStyle', ':', 'LineWidth',1, 'Color', [0 0 0]);

line(D1LineH(1,:), D1LineH(2,:), 'LineStyle', ':', 'LineWidth',1, 'Color', [0 0 0 $]$ );

line(D2LineH(1,:), D2LineH(2,:), 'LineStyle', ':', 'LineWidth',1, 'Color', [0 0 0]);

line(D12LineH(1,:), D12LineH(2,:), 'LineStyle', ':', 'LineWidth',1, 'Color', [0 0 0]);

axis( AxisRange ); colormap jet;

title('Imaginary');

saveas(gcf, strcat(OutDataPath, 'Fig7'), 'emf');

Save 2D plot in 600dpi png format

print(gcf, '-r600', '-dpng', '-noui', strcat(OutDataPath, 'Fig7', '.png'));

$\%$

fig8 = figure(8);

dh = datacursormode(fig8);

set(dh,'SnapToDataVertex','off','UpdateFcn',@mydatatip);

\%set(gcf, 'Units', 'inch');

\%set(gcf, 'position', [ 2167.5 ]);

\%subplot(211);

\%plot(specenergy, RefSpec_THz/max(RefSpec_THz), 'b-');

\%axis( AxisRange1 );

\%set(gca, 'Units', 'inch');

\%set(gca, 'position', [ 0.55 .551 .5 ]);

\%subplot(212);

$\% \operatorname{VMax}=1.0$;

hFig = contour(gEmiFreq, gAbsFreq, MAmpl, linspace(0, VMax, NContourLevels), 'LineWidth', 1.5);

\% hFig = contour(gEmiFreq, gAbsFreq, MReal, linspace(-VMax, VMax, NContourLevels), 'LineWidth', 1.5);

axis( AxisRange );

line(DiagnILine(1,:), DiagnILine(2,:), 'LineStyle', ':', 'Color', [0 0 0 $]$ );

colormap jet;

set(gca, 'Units', 'inch');

\%set(gca, 'position', [ 0.50 .555 ]);

xlabel('Emission (meV)');

ylabel('Absorption (meV)');

\%title('GaAs Multiple Quantum Well');

saveas(gcf, strcat(OutDataPath, 'Fig8_Ampl'), 'emf'); 
$\%$

fig9 = figure(9);

set(gcf, 'Units', 'inch');

set(gcf, 'position', [ $\left.\begin{array}{lllll}2 & 1 & 6 & 7.5\end{array}\right]$ );

subplot(211);

plot(specenergy, RefSpec_THz/max(RefSpec_THz), 'b-');

axis( AxisRange1);

set(gca, 'Units', 'inch');

set(gca, 'position', [ 0.55 .551 .5 ]);

subplot(212);

$\% \operatorname{VMax}=1.0$;

\%hFig = contour(gEmiFreq, gAbsFreq, MAmpl, linspace(0, VMax, NContourLevels), 'LineWidth', 1.5);

hFig = contour(gEmiFreq, gAbsFreq, MReal, linspace(-VMax, VMax, 3*NContourLevels), 'LineWidth', 1.5);

axis( AxisRange );

line(DiagnILine(1,:), DiagnILine(2,:), 'LineStyle', ':', 'Color', [0 0 0 $]$ );

colormap jet;

set(gca, 'Units', 'inch');

set(gca, 'position', [ 0.50 .555 ]);

saveas(gcf, strcat(OutDataPath, 'Fig9_Ampl'), 'emf');

\section{A.12 Calculate “Camera Phase" (lineout.m)}

$\% \%$ read and plot lineouts extracted from focus position camera

clear all; clc; tic;

y0=2; $a 1=1 ; a 2$ = 1; w0 = 0.109;

$\% \%$ files

FFindex = '22';

FPath = strcat('.\2D',FFindex, '\alloptical\');

FName1 = 'TopUnlocked'; FName2 = 'TopLocked';

FName3 = 'BottomUnlocked'; FName4 = 'BottomLocked';

raw1 = dlmread(strcat(FPath, FName1, '.dat'), '\t');

raw2 = dlmread(strcat(FPath, FName2, '.dat'), '\t');

raw3 = dlmread(strcat(FPath, FName3, '.dat'), '\t');

raw4 = dlmread(strcat(FPath, FName4, '.dat'), '\t');

$\% \%$ truncate patterns

ncut1 = 1; ncut2 = ncut1;

ctarry = [ncut1 : size $($ raw1 $(:, 1), 1)$ - ncut2]';

$\mathrm{m}=$ size(ctarry,1);

data1 = raw1 $($ ctarry,1);

data2 $=$ raw2 $($ ctarry, 1$) ;$

data3 = raw3(ctarry,1); 


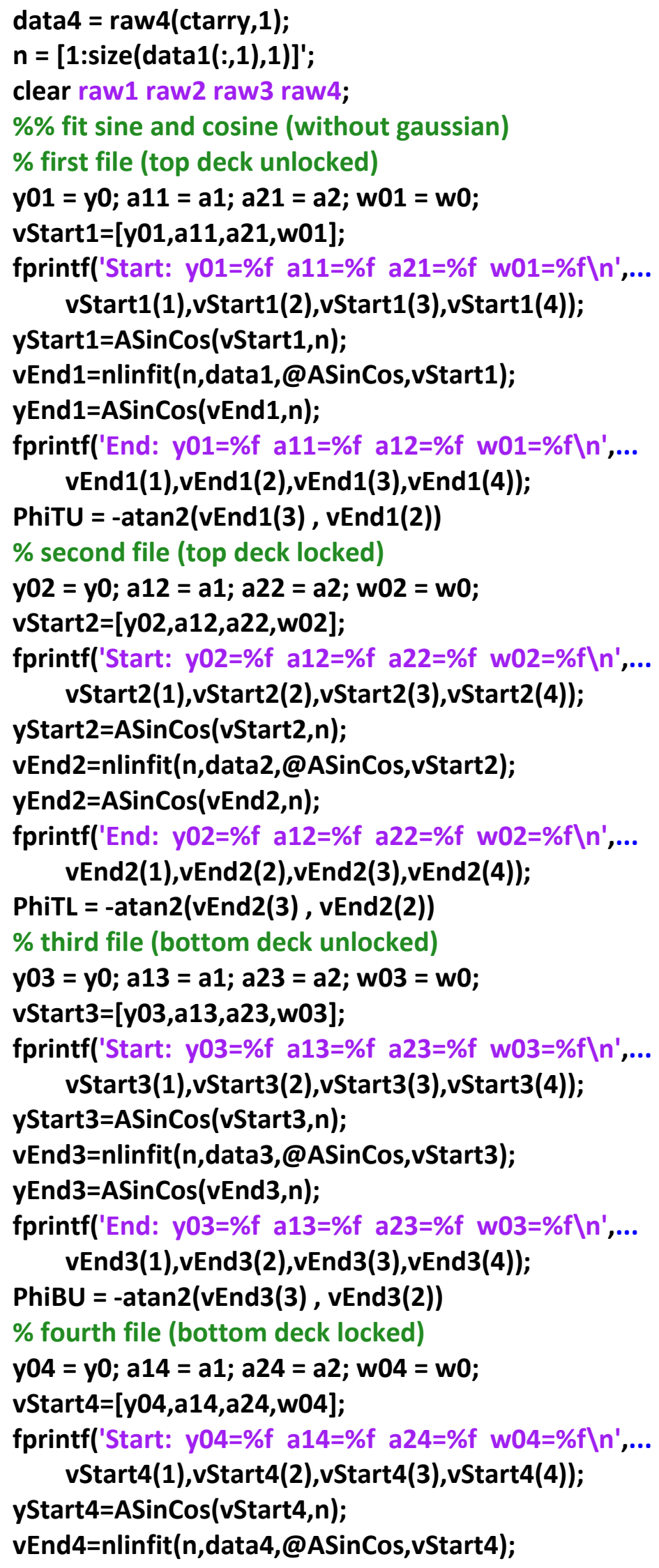




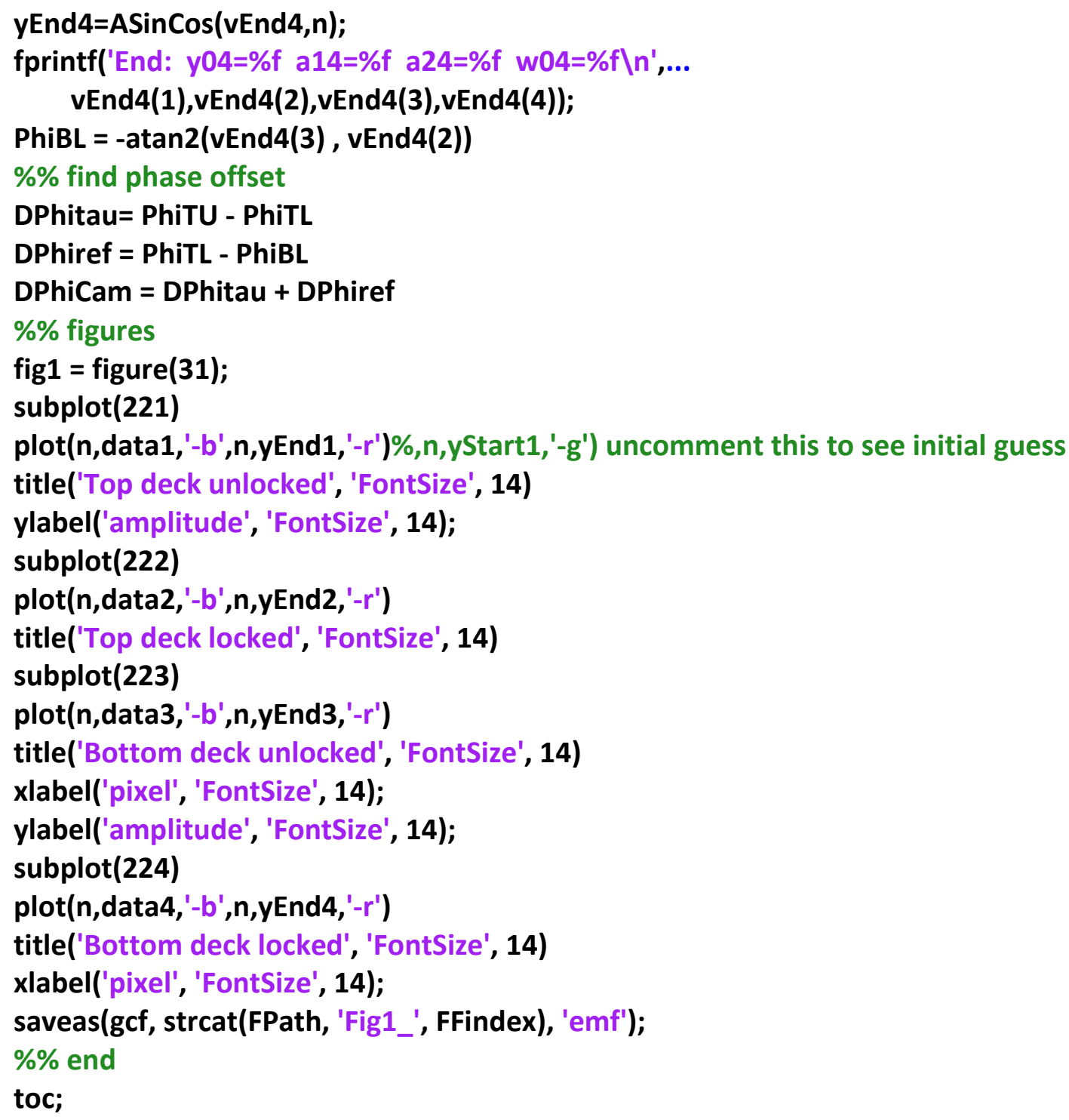

A.13 Linear Absorption and Ideal Resonance Linehsapes code (LinAbsorption2.m) $\% \%$ program to compute linear absorption from spectra 


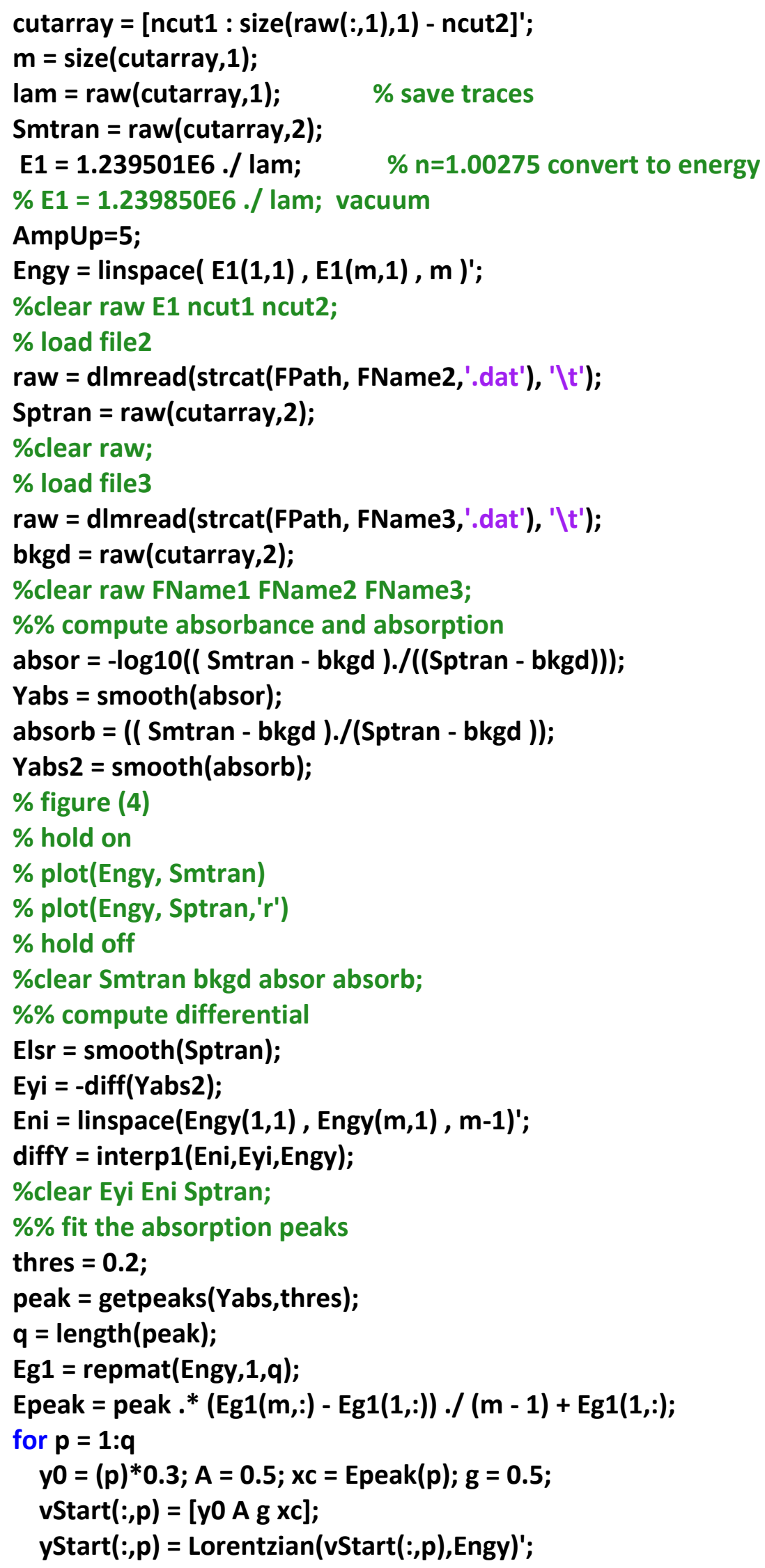




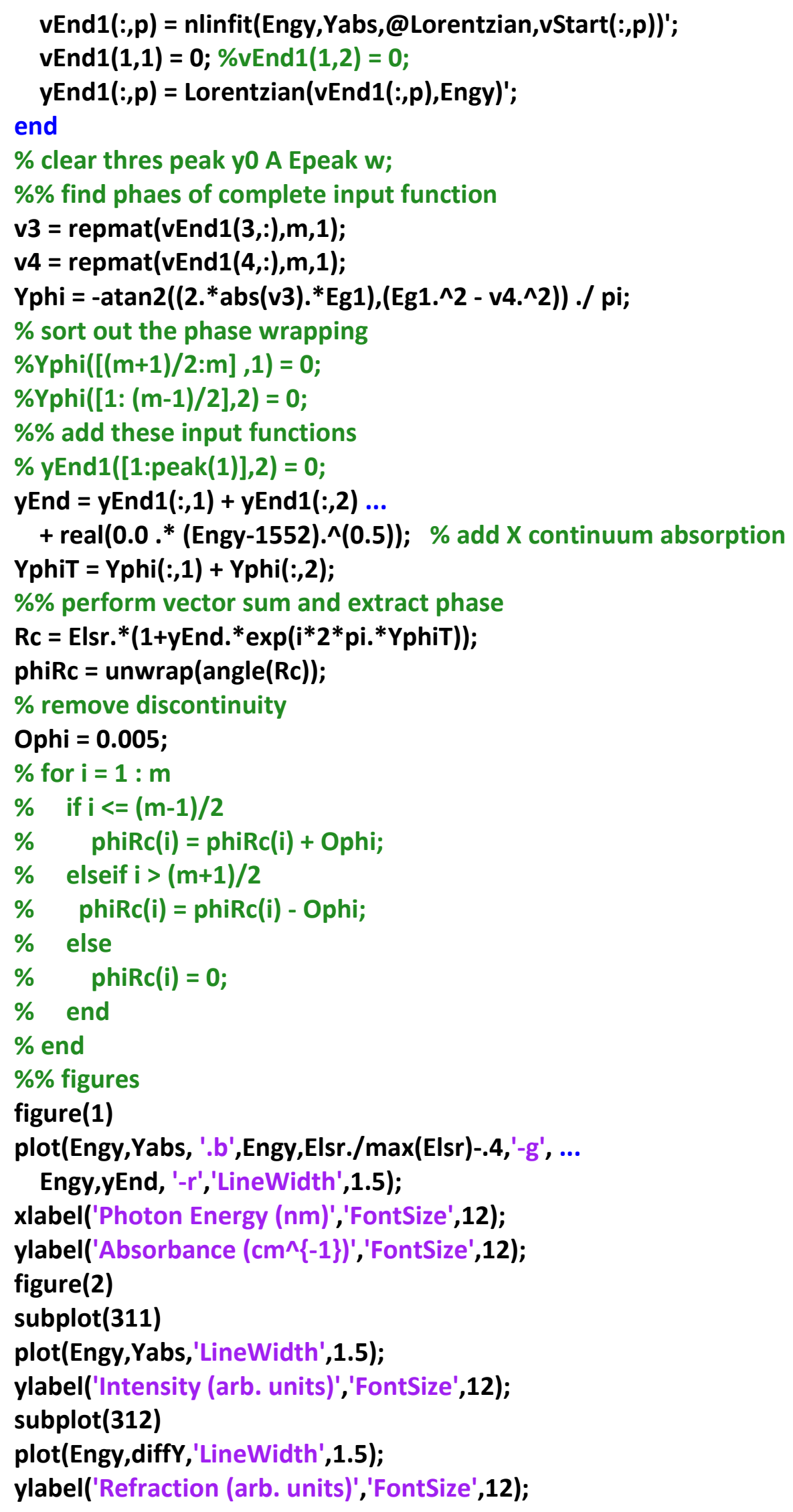


subplot(313)

plot(Engy,YphiT, 'LineWidth',1.5);

xlabel('Photon Energy (nm)','FontSize',12);

ylabel('Phase (?)','FontSize',12);

figure(3)

subplot(211);

plot(Engy, real(Rc),Engy,imag(Rc),'LineWidth',1.5)

legend('real','imag');

subplot(212);

plot(Engy,phiRc,'-b', Engy,diffY.*AmpUp, ':b','LineWidth',1.5)

legend('phiRc','diffY*AmpUp')

$\% \%$ save files

data(:,1) = Engy; data(:,2) = phiRc;

data2(:,1) = Engy; $\operatorname{data2}(:, 2)$ = Yabs;

dlmwrite('phi_vsum.dat', data, '\t');

dlmwrite(strcat('absorbance0',num2str(FFindex),'.dat'), data2, 'ไt');

$\%$ saveas(gcf, strcat(FPath, 'AbsorbFig'), 'emf');

\% print(gcf, '-r600', '-dpng', '-noui', strcat(FPath, 'AbsorbFig', '.png'));

$\% \%$ end

\%\%Absorption coefficient calculation

abs_coeff $=\log (1.3 *$ Sptran./(Smtran $)) / 1$;

figure(4)

plot(Engy,abs_coeff);

title('absorption coefficient per micron')

toc;

\section{A.14 Tracer/SI Phase Code (TraserSI.m)}

$\% \%$ reads spectra of Tracer, Reference and their interference

format long g; clear all; clc; tic;

$\% \%$ constants and control parameters:

SpeedC $=2.99792458 \mathrm{E}+5 / 1.000275 ; \%$ Speed C $=2.99702547 \mathrm{E}+5 ; \quad \% \mathrm{~nm} / \mathrm{ps}$

Frq2Egy $=4.135667516 ; \%$ Frq2Egy $=4.1356673 ; \quad \%$ conversion from $\mathrm{THz}$ to $\mathrm{meV}$

ScanIndex = '2';

$\%$ Wavelengths of SPEX-750M spectrometer

WLMin = 811.569; WLMax = 831.661; \% nm

NEmiDim = 1024;

$\%$ plot size

FreqLowLmt $=362 ;$ FreqHiLmt $=367 ; \% \mathrm{THz} \%$ length of the emission dimension (CCD length) IndexLowLmt = round(((FreqLowLmt/SpeedC-1/WLMax)*NEmiDim)/(1/WLMin-1/WLMax)); IndexHiLmt = round(((FreqHiLmt/SpeedC-1/WLMax)*NEmiDim)/(1/WLMin-1/WLMax)); Multipier = 1.0;

NFirstCol =round(IndexLowLmt - Multipier*(IndexHiLmt-IndexLowLmt)/2.0);

NLastCol = round(IndexHiLmt + Multipier*(IndexHiLmt-IndexLowLmt)/2.0);

$\%$ NFirstCol $=160 ; \quad$ NLastCol $=900$; 
if (NFirstCol < 1) NFirstCol = 1; end

if (NLastCol > 1024) NLastCol = 1024; end

P2DLowEngyLmt = FreqLowLmt * Frq2Egy; P2DHiEngyLmt = FreqHiLmt * Frq2Egy;

$\%$ Cut off residual part near time zero after IFT TRA, Unit: index

TimeCut $=5.2 ; \quad \%$ ps

TimeCutAfter $=9$;

$\%$ delay between third pulse and reference

DelayTau0 $=4.82315 ; \quad \%$ ps

$\%$ phase offset for phidata file: Ophi = linear offset,

$\%$... phi2 = quadratic amplitude, $\mathrm{Em0}=$ quadratic center,

$\%$... Freqshift $=$ shift of phi_vsum to match current data

$\%$ Ophi $=-3.74$; phi2 = .032; Em0 = 363.58; FrqShft $=-0.1$;

Ophi = -1.52; phi2 = .0265; Em0 = $363.30 ;$ FrqShft = -0.1;

$\%$ uses fit value if usefittime $=1$, else it does not

usefittime = 1;

$\% \%$ Data Path and Filenames:

DataPath = strcat('.\2D',Scanlndex, '\');

TRAfname = 'tra00.dat';

SIfname = 'traref00.dat';

Reffname = 'ref00.dat';

phi_datdat = 'phi_vsum.dat';

TRASpec $=$ dlmread(strcat $($ DataPath, TRAfname $)$, ' $(t ')$;

TRASpec = flipud $($ TRASpec $)$;

SISpec = dlmread(strcat(DataPath, SIfname), '\t');

SISpec = flipud(SISpec);

RefSpec = dlmread(strcat(DataPath, Reffname), '\t');

RefSpec $=$ flipud $($ RefSpec $) ; \quad \%$ flip array up-down

OutDataPath = strcat(DataPath, 'alloptical\');

if isdir(OutDataPath) mkdir(DataPath, 'alloptical'); end

$\% \%$ reformats to $\mathrm{THz}$

TRASpec = TRASpec; \%- $\operatorname{sum}([\operatorname{TRASpec}(1: 10) ; \operatorname{TRASpec}(1015:$ end $)]) / 20 \%$ removing the background counts

SISpec = SISpec; \%- sum([SISpec(1:10); SISpec(1015:end) $]) / 20$

InterfSpec = SISpec - TRASpec - RefSpec;

[RefSpec_THz, FreqMin, FreqMax] = resampleWL2Freq(RefSpec, WLMin, WLMax, NEmiDim);

[TRASpec_THz, FreqMin, FreqMax] = resampleWL2Freq(TRASpec, WLMin, WLMax, NEmiDim);

[SISpec_THz, FreqMin, FreqMax] = resampleWL2Freq(SISpec, WLMin, WLMax, NEmiDim);

[InterfSpec_THz, FreqMin, FreqMax] = resampleWL2Freq(InterfSpec, WLMin, WLMax, NEmiDim);

$\%$ create frequency axis

FreqAxis = transpose(linspace(FreqMin, FreqMax, NEmiDim));

dlmwrite( strcat(OutDataPath, 'TRA_Ref_InterfSpec.dat'),...

[FreqAxis TRASpec_THz RefSpec_THz InterfSpec_THz], ' \t'); 
$\% \%$ Plot the spectra of Tracer, Ref and Interferogram

fig1 = figure(21);

subplot(211); \% input spectra

plot(FreqAxis, RefSpec_THz, 'b-', FreqAxis,TRASpec_THz*5, 'r-', FreqAxis, SISpec_THz, 'k-');

AxisScale = axis; axis([ FreqLowLmt-1 FreqHiLmt+1 AxisScale(3:4) ]);

xlabel('Emission Frequency (THz)', 'FontSize', 10);

ylabel('Spectral Intensity (a.u.)', 'FontSize', 10);

title('Spectra of Tracer(red), Ref(blue) and their interferogram(blk)', 'FontSize', 10);

subplot(212); \% determined interference term

plot(FreqAxis, InterfSpec_THz, 'k-');

AxisScale = axis; axis([ FreqLowLmt-1 FreqHiLmt+1 AxisScale(3:4) ]);

xlabel('Emission Frequency (THz)', 'FontSize', 10);

ylabel('Spectral Intensity (a.u.)', 'FontSize', 10);

title('Interferometric Term', 'FontSize', 10);

saveas(gcf, strcat(OutDataPath, 'Fig21'), 'emf');

$\% \%$ Inverse Fourier transform to time domain (use FFT)

IFT_InterfSpec $=\mathrm{fft}(\mathrm{fftshift}($ InterfSpec_THz, 1), NEmiDim, 1);

IFT_InterfSpec1 = IFT_InterfSpec;

$\%$ figure(33)

$\%$ plot(abs(IFT_InterfSpec1));

$\%$ Cut off residual part near time zero

NEmilnitCut = round(TimeCut*(FreqMax-FreqMin));

NEmilnitCut2 =round(TimeCutAfter*(FreqMax-FreqMin));

IFT_InterfSpec1(1: NEmilnitCut) = 0;

IFT_InterfSpec1(NEmilnitCut2 : 1024) = 0;

$\%$ remove whole section from incorrect time ordering

IFT_InterfSpec1(NEmiDim/2+1 : NEmiDim) = 0;

$\% \%$ fit main peak to find t-zero

RTimeAxis = linspace( 0, (NEmiDim-1)/(FreqMax-FreqMin), NEmiDim );

$\%$ thres = 1e3;

\%peak = getpeaks(abs(IFT_InterfSpec1),thres);

[peak,loc] = findpeaks(abs(IFT_InterfSpec1),'minpeakdistance',5E2);

Rpeak1 = loc./(FreqMax-FreqMin);

y0 = 0; $A=1 \mathrm{e} 5 ; \mathrm{xc}=$ Rpeak1; $w=0.1$;

vStart $=$ [y0 A xc w];

yStart = Gaussian(vStart,RTimeAxis)';

vEnd = nlinfit(RTimeAxis,abs(IFT_InterfSpec1)',@Gaussian,vStart)';

IFT_Fit = Gaussian(vEnd,RTimeAxis)';

if usefittime $==1$

DelayTau0 $=$ vEnd(4)

end

$\% \%$ plot the time-domain 
fig2 = figure(22);

plot( RTimeAxis, abs(IFT_InterfSpec1), 'g-', RTimeAxis, IFT_Fit, 'r-');

\%axis([0 RTimeAxis(end)/2 0 1.1*max(abs(IFT_InterfSpec1)) ]);

xlabel('Real Emission Time (ps)', 'FontSize', 10);

ylabel('Intensity (a.u.)', 'FontSize', 10);

title('IFTed interferogram', 'FontSize', 10);

dlmwrite( strcat(OutDataPath, 'realtime_IFT.dat'),...

[RTimeAxis' abs(IFT_InterfSpec) abs(IFT_InterfSpec1)], 'It');

saveas(gcf, strcat(OutDataPath, 'Fig22'), 'emf');

$\% \%$ Fourier transform back to spectral domain (use IFFT)

FT1 = ifft(IFT_InterfSpec1, NEmiDim, 1);

FT1Shift = ifftshift(FT1, 1);

$\%$ calculate retrieved signal

RetrTRA = FT1Shift ./ sqrt(RefSpec_THz);

$\% \%$ find phase

PhaseRetrTRA = angle( RetrTRA .* $\exp \left(-i * 2^{*}\right.$ pi* FreqAxis .* DelayTau0) $)$;

\% PhaseRetrTRA1 = unwrap(angle( $\exp (i$ * PhaseRetrTRA(NFirstCol:NLastCol))));

PhaseRetrTRA1 = unwrap(PhaseRetrTRA(NFirstCol:NLastCol));

RetrTRA1 = RetrTRA(NFirstCol:NLastCol);

TRASpec_THz1 = TRASpec_THz(NFirstCol:NLastCol);

FreqAxis1 = FreqAxis(NFirstCol:NLastCol);

dlmwrite( strcat(OutDataPath, 'RetrTRAefield.dat'), ...

[FreqAxis abs(RetrTRA) PhaseRetrTRA], 'It');

$\% \%$ load phase from vector sum (see 2008_08_01 \abstec)

phidata = load(strcat(DataPath, phi_datdat));

phidata(:,1) = phidata(:,1) ./ Frq2Egy + FrqShft;

phidata(:,2) = phidata(:,2) + Ophi + phi2.*( phidata(:,1) - Em0 ).^2;

dlmwrite( strcat(OutDataPath, 'phasefind.dat'), phidata, ' 't');

$\% \%$ plot retrieved signal for spectrum and phase

fig3 = figure(23);

subplot(211);

\% This is the factor used to normalize the 2D data

MaxSRTRAAmp $=\max ($ abs $($ RetrTRA1));

plot(FreqAxis1, abs(RetrTRA1.^2)./max(abs(RetrTRA1.^2)), 'r-', ...

FreqAxis1, TRASpec_THz1./max(TRASpec_THz1), 'k-');

AxisScale = axis; axis([ FreqLowLmt FreqHiLmt 01.1$])$;

xlabel('Emission Frequency (THz)', 'FontSize', 10);

ylabel('Intensity (a.u.)', 'FontSize', 10);

title('Retrieved(Red) \& Measured(Blk) Tracer Intensity', 'FontSize', 10);

subplot(212);

IndexLowLmt1 = round(IndexLowLmt + NFirstCol);

IndexHiLmt1 = round(IndexHiLmt - NFirstCol);

$\% \operatorname{plot}($ FreqAxis1, PhaseRetrTRA1, 'r');

plot(FreqAxis1, smooth(smooth(PhaseRetrTRA1)), 'r', ... 
phidata(:,1),phidata(:,2), 'b', 'LineWidth', 1.5);

$\min$ (PhaseRetrTRA1)

axis([ FreqLowLmt FreqHiLmt...

min(PhaseRetrTRA1(IndexLowLmt1:IndexHiLmt1))-pi/8 ...

max(PhaseRetrTRA1(IndexLowLmt1:IndexHiLmt1))+pi/8]);

xlabel('Emission Frequency (THz)', 'FontSize', 10);

ylabel('Ripped Phase (rad.)', 'FontSize', 10);

title('Retrieved Tracer Phase, unwrapped', 'FontSize', 10);

dlmwrite( strcat(OutDataPath, 'RetrTRAefield1.dat'), ...

[FreqAxis1 abs(RetrTRA1) PhaseRetrTRA1], '(t');

saveas(gcf, strcat(OutDataPath, 'Fig23'), 'bmp');

A.15 Peak Fitting Code (Linewidth_coupledfitCleaned.m)

clear all;clc;

\%outpaths, one for the fit parameters other for slices

FpathPara='. |Fits \';FpathSlices='. \Fits \Raw and Fit Slices $\backslash$ ';

$\mathbf{y}=\mathbf{2}$;

constant $=1$;

FIndexStart $=1 ; \%$ Specify start scan index

FIndexEnd $=64 ; \%$ Specify last scan index

PeakName $=[$ 'A' 'C'];

for $m=$ FIndexStart:FIndexEnd;

for peakindex = 1:y;

FileSuff = PeakName(1, peakindex);

FFindex $=$ num2str $(m)$;

FPath = strcat('. (','2D',FFindex, '\Output $\backslash$ ');

FName = 'MAmpl.dat';

FRangeName = 'MFreqRange.txt';

$\%$ number of contour lines to be used

NContourLevels = 35;

$\%$ thickness of contour lines

ContourlineWidth = 1.0;

$\%$ the num of points representing diag or cross-diag line

LineLength = 1000;

if FileSuff $=={ }^{\prime} A$ '

MLowLmt=1489.8;

MHiLmt=1491.5;

else

MLowLmt=1494;

MHiLmt=1496;

end

fid = fopen( strcat(FPath, FRangeName), 'r');

while 1

TxtLine = fgetl(fid); 


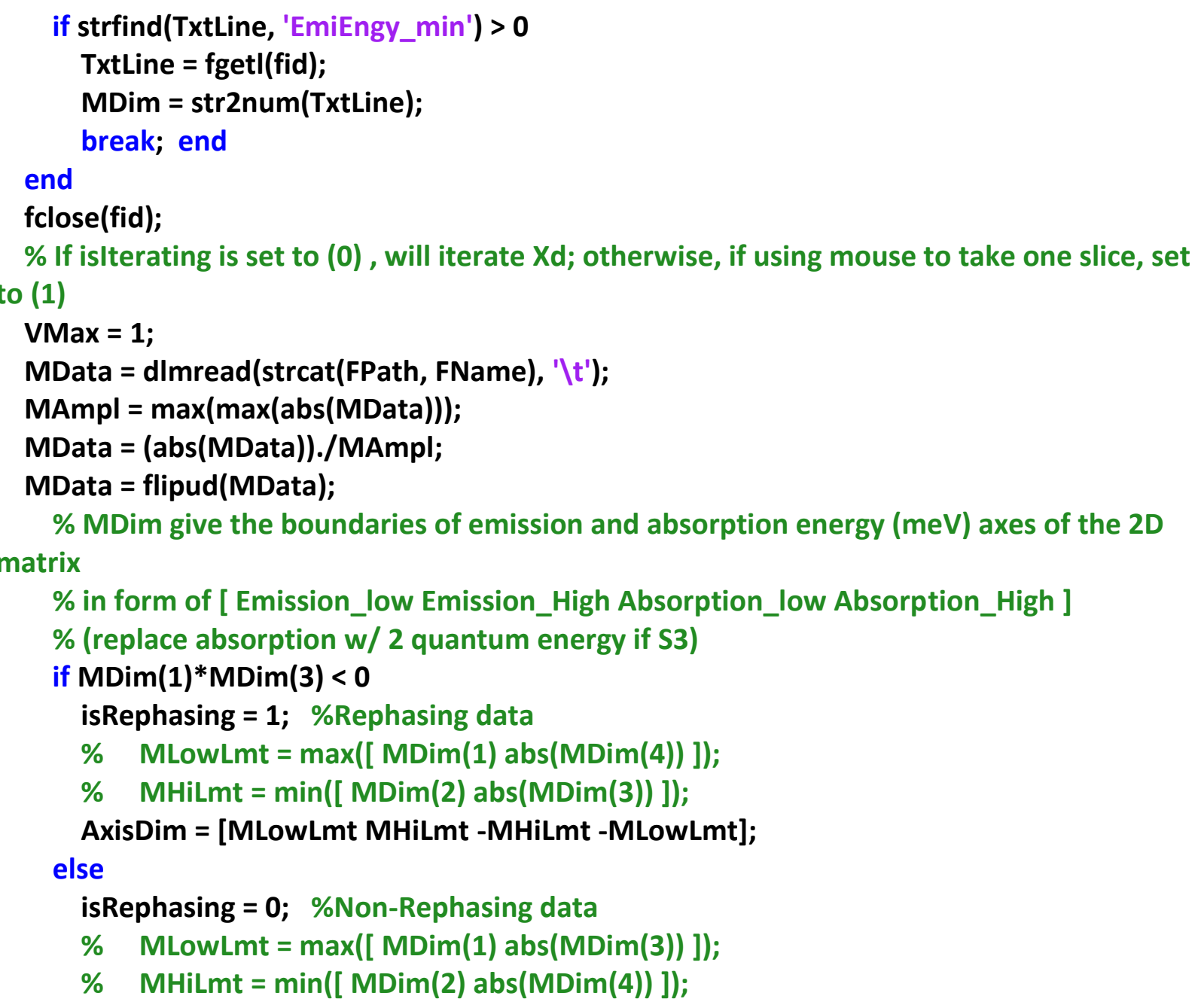

AxisDim = [MLowLmt MHiLmt MLowLmt MHiLmt];

end

$\%$ Plot Amp, real and imaginary parts of 2D data

NRow = size(MData, 1); NCol = size(MData, 2);

$\%$ Meshgrid: gEmiFreq contains rows of first vector (\#rows = length of second vector); gAbsFreq contains

$\%$ columns of second vector (\#columns = length of first vector)

[gEmiFreq, gAbsFreq] = meshgrid( $\operatorname{single}(\operatorname{linspace}(\operatorname{MDim}(1), \operatorname{MDim}(2), \mathbf{N C o l})), \ldots$

single(linspace(MDim(4), MDim(3), NRow)));

$\% \%$ Plot 2D Amplitude Spectrum

fig1 = figure(11);

set(fig1, 'Position', [ 50100700660 ]);

$\% \operatorname{MData}($ NRow, NCol-1)= -1; MData(NRow, NCol)= 1;

contour(gEmiFreq, gAbsFreq, MData, linspace(0, VMax, NContourLevels), 'LineWidth', ContourlineWidth);

axis(AxisDim);

axis square; colormap jet; 
DiagX = linspace( MLowLmt, MHiLmt, LineLength );

DiagY = -DiagX;

line(DiagX, DiagY, 'LineWidth', 1, 'LineStyle', '-', 'Color', [ $\left.\left.\begin{array}{lll}0 & 0 & 0\end{array}\right]\right) ;$

hold on

$\%$

\% Determine Data Sampling Range (taking slices along diagonal)

figure(fig1);

datacursormode on;

dcm_obj = datacursormode(fig1);

set(dcm_obj,' 'DisplayStyle','window','SnapToDataVertex','off');

$\%$ Curlnfo = getCursorInfo(dcm_obj);

$\%[X p Y p]=$ Curlnfo.Position;

$\%$ mouse cursor point at any random position

[Xp, Yp, MouseButton]= ginput(1);

if MouseButton =1 break; end

$X M a x=Y p+X p+$ MHiLmt;

YMin = -MLowLmt + Yp + Xp;

diagrangeX = linspace(MLowLmt, XMax, LineLength);

diagrangeY = linspace(YMin, -MHiLmt, LineLength);

line(diagrangeX, diagrangeY, 'LineWidth', 1, 'LineStyle', ':', 'Color', [ $\left.\begin{array}{ll}0 & 0\end{array}\right]$ );

XcrossMax = -MLowLmt + Xp - Yp;

YcrossMin = MLowLmt $-X p+Y p ;$

crossdiagrangeX = linspace(MLowLmt, XcrossMax, LineLength);

crossdiagrangeY = linspace(YcrossMin, -MLowLmt, LineLength);

line(crossdiagrangeX, crossdiagrangeY, 'LineWidth', 1, 'LineStyle', ':', 'Color', [ $\left.\begin{array}{ll}0 & 0\end{array}\right]$ );

hold off

$\% \%$ Sample Z values along the diagonal line going through $(X p, Y p)$

DiagDist = (diagrangeX-Xp); \% MLowLmt is used as the beginning of diagonal line

DiagZ2D = interp2(gEmiFreq, gAbsFreq, MData, diagrangeX, transpose(diagrangeY), 'linear');

DiagZ2D = single(DiagZ2D);

DiagZ_0L = zeros(1, LineLength);

for idx = 1: LineLength

DiagZ_OL(idx) = DiagZ2D(idx, idx);

end

DiagZ_1L = zeros(1, LineLength);

for idx = 2: (LineLength-1)

DiagZ_1L(idx) $=($ DiagZ2D(idx+1, idx-1) + DiagZ2D(idx-1, idx+1))/2;

end

DiagZ_2L = zeros(1, LineLength);

for idx = 3: (LineLength-2) 
DiagZ_2L(idx) $=(\operatorname{DiagZ2D}(i d x+2, i d x-2)+\operatorname{DiagZ2D}(i d x-2, i d x+2)) / 2 ;$

end

DiagZ = ( DiagZ_OL + DiagZ_1L + DiagZ_2L )/3; \% Binning 5 lines

$\%$ Determine peak of inhomogeneous profile and its location.

DiagMax = $\max ($ DiagZ_OL);

I = 1;

while DiagZ_OL(I) < DiagMax

I = I+1;

end

PeakLocation $=$ DiagDist + Xp;

$\% \%$ Sample Z values along the cross diagonal line through (Xp,Yp)

CrossDiagDist $=$ (crossdiagrangeX-Xp); \% MLowLmt is used as the beginning of cross diagonal line

CrossDiagZ2D = interp2(gEmiFreq, gAbsFreq, MData, crossdiagrangeX, transpose(crossdiagrangeY), 'linear');

CrossDiagZ2D = single(CrossDiagZ2D);

CrossDiagZ_0L = zeros(1, LineLength);

for idx = 1: LineLength

CrossDiagZ_0L(idx) = CrossDiagZ2D(idx, idx);

end

CrossDiagZ_1L = zeros(1, LineLength);

for idx = 2: (LineLength-1)

CrossDiagZ_1L(idx) = (CrossDiagZ2D(idx+1, idx-1) + CrossDiagZ2D(idx-1, idx+1))/2;

end

CrossDiagZ_2L = zeros(1, LineLength);

for idx = 3: (LineLength-2)

CrossDiagZ_2L(idx) = (CrossDiagZ2D(idx+2, idx-2) + CrossDiagZ2D(idx-2, idx+2))/2; end

CrossDiagZ = $($ CrossDiagZ_OL + CrossDiagZ_1L + CrossDiagZ_2L )/3; \% Binning 5 lines $\%$ Determine region of CrossDiagZ used for fit

CrossMax = $\max ($ CrossDiagZ_OL);

CrossOffset $=($ sum(CrossDiagZ_0L(1:10))+sum(CrossDiagZ_0L(length(CrossDiagZ_0L)9:length(CrossDiagZ_OL))))/20;

CrossFWHM = CrossMax- $(($ CrossMax-CrossOffset $) / 2)$;

$\mathrm{j}=1$;

while CrossDiagZ_OL(j) < CrossFWHM

$$
\text { j= j+1; }
$$

end

$k=1$;

while CrossDiagZ_OL(j+k) > CrossFWHM

$$
k=k+1 \text {; }
$$

end 
CrossDiagZFitValues = CrossDiagZ_0L(j:j+k-1);

CrossDiagDistFitValues = CrossDiagDist $(j: j+k-1)$;

$\% \quad$ BothSlices $=$ [DiagZ_OL' CrossDiagZ_OL']'; \%BLW version

$\% \quad$ BothSlicesX = [DiagDist' CrossDiagDist']';

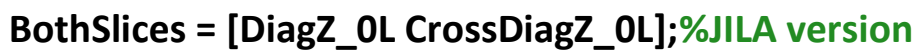

BothSlicesX = [DiagDist' CrossDiagDist']';

\%The elements of T02 are (1)Ho Gamma(cross) (2) Inho Gamma(diag)

$\%(3)$ Amplitude diag (4) horiz offset (5) vert offset (6)Amplitude Cross

$\%$ diag (7) is cross diag horiz offset

$\% \quad 1234567$

$\% \quad$ TO2start = [. $\left.4 \begin{array}{llllll}1 & 1 & 0 & 0 & 1 & 0\end{array}\right]$;

$\% \quad$ TO2start $=[.19 .05 .6 .001-.014 .10-.005]$;

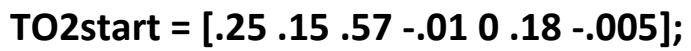

\%T02final = nlinfit(BothSlicesX, BothSlices, @T02X0, T02start)

$\% \%$ Figure plots the slices and initial guesses

TO2fit = TO2X0(TO2start, BothSlicesX );

fig2 = figure(5);

clf(fig2)

subplot $(2,1,1)$

hold on

plot(BothSlicesX(2,:),BothSlices(1001:2000))

plot(CrossDiagDist, TO2fit(length(DiagDist)+1:length(DiagDist)+length(CrossDiagDist)), '$\left.r^{\prime}\right)$;

hold off

title('Initial Guesses: Cross-Diag');

subplot $(2,1,2)$

hold on

plot(BothSlicesX(1,:),BothSlices(1:1000))

plot(DiagDist, T02fit(1:length(DiagDist)), '-r')

hold off

title('Diag slice');

[TO2final, Residue, Jacob] = nlinfit(BothSlicesX, BothSlices, @TO2X0, T02start);

TO2inter = nlparci(TO2final,Residue, 'jacobian',Jacob);

TO2ParaEr1 = (TO2inter(1,2)-TO2inter(1,1))/2;

TO2ParaEr2 = (TO2inter(2,2)-TO2inter(2,1))/2;

T02fit = TO2X0( TO2final, BothSlicesX $)$;

TO2fitDiag = TO2fit(1:length(DiagDist));

TO2fitCrossDiag = TO2fit(length(DiagDist)+1:length(DiagDist)+length(CrossDiagDist));

TO2fitErr = sqrt( $1 /(2 *$ length $($ CrossDiagZ_0L $)-1) * .$.

(sum(abs(DiagZ_OL-TO2fitDiag).^2) + sum(abs(CrossDiagZ_0L-TO2fitCrossDiag).^2))); 


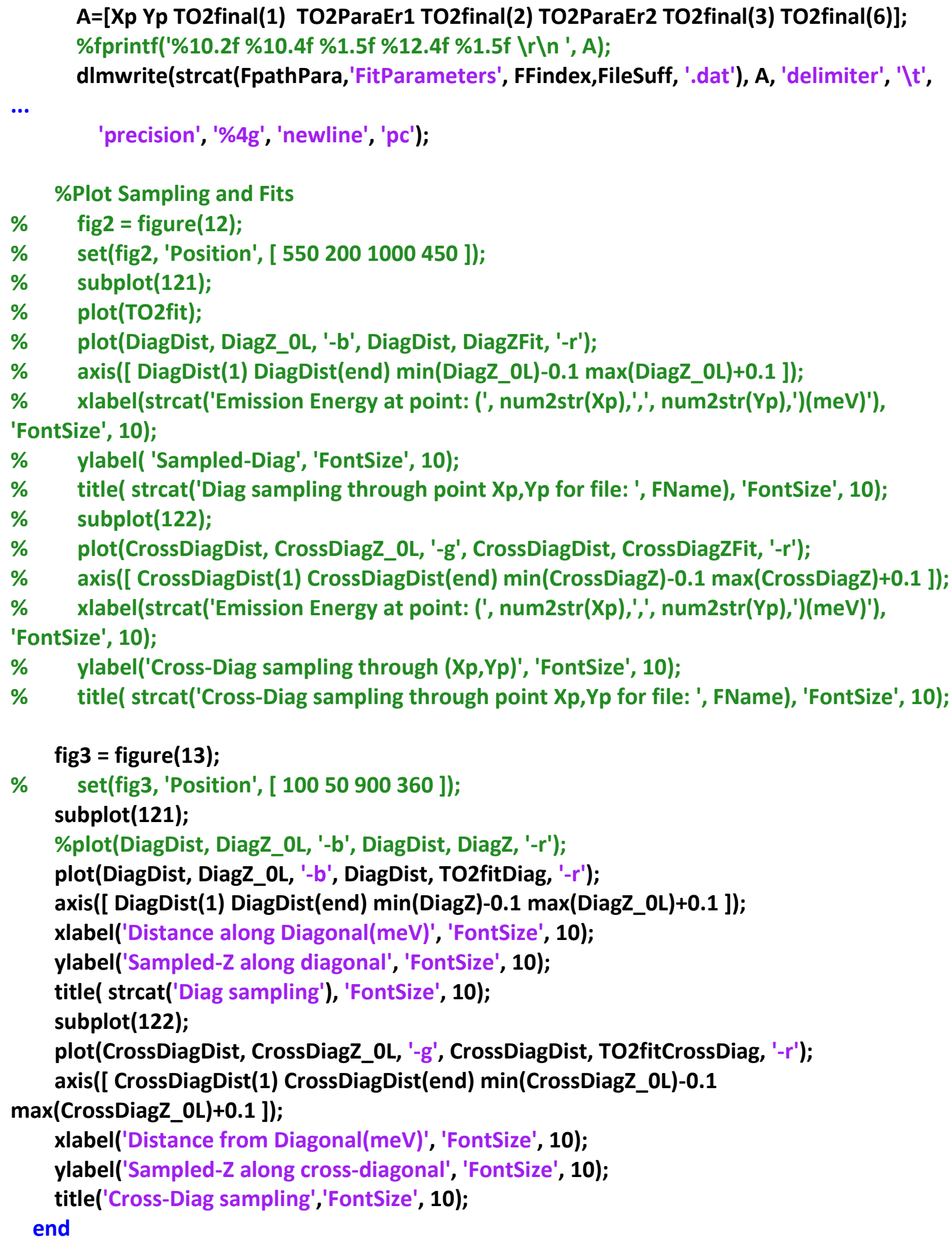


dlmwrite(strcat(FpathSlices, 'DiagSlices',FFindex,FileSuff, '.dat'), [DiagDist' DiagZ_OL' TO2fitDiag'], 'delimiter', '\t', ... 'precision', '\%4g', 'newline', 'pc');

dlmwrite(strcat(FpathSlices, 'CrossDiagSlices',FFindex,FileSuff, '.dat'), [CrossDiagDist' CrossDiagZ_0L' TO2fitCrossDiag'], 'delimiter', '\t', ... 'precision', '\%4g', 'newline', 'pc');

end

\section{A.16 Peak Integration Code (integrateregion2.m)}

$\% \%$ Reads in peak positions of populations from peak fits for where to place boxes

clear all; clc;

FIndexStart $=1 ; \%$ Specify start scan index

FIndexEnd $=64 ; \%$ Specify last scan index

mkdir('. IIntegrated Data\')

FOutPath = '. IIntegrated Datal';

for $\mathbf{m}=$ FIndexStart:FIndexEnd;

clc

FFIndex = num 2str(m);

FPath = strcat('.|2D',FFIndex, '\Output $\backslash$ ');

FName $=$ 'MAmpl.dat';

FRangeName $=$ 'MFreqRange.txt';

FNamepk1 = strcat('.|Fits \FitParameters',FFIndex,'A.dat');

FNamepk2 = strcat('.|Fits $\backslash$ FitParameters',FFIndex, 'B.dat');

$\%$ if $X d=1$--> user defined values; $X d=0$--> pick points using mouse

$\mathrm{Xd}=\mathbf{1}$;

$\%$ number of contour lines to be used

NContourLevels $=30$;

$\%$ thickness of contour lines

ContourlineWidth = 1.0;

$\%$ the num of points representing diag or cross-diag line

LineLength $=200$;

MLowLmt=1475; \% low energy plotting range

MHiLmt=1502; \% high energy plotting range

Peak1Info $=1490.3 ; \% d l$ mread(strcat(FNamepk1), ' $\backslash t '$ ');

Peak2Info = 1494.8;\%dlmread(strcat(FNamepk2), ' $\left(t^{\prime}\right)$;

Lo_energy_pk = Peak1Info(1);\%Lo energy pk

Hi_energy_pk = Peak2Info(1);\%Hi energy pk 
\% Hi_energy_pk = 1496.19;\%Hi energy pk

\% Lo_energy_pk = 1490.69;\%Lo energy pk

\%\% Peak Analyzing: choose which peak to analyze

recalculate $=\mathbf{1}$;

$\%$ This choice will determine RangeValues within which the peak lies. If $\%$ recalculate $=0$, then will integrate the user defined region, find the $\%$ maximum peak value in this region, and then re-integrate within a region $\%$ of the same size but centered about the peak.

$\%+/-$ tau

$y=1.5$

$\%+/-\mathrm{t}$

$x=1.5$;

for $z=1: 4$;

if $z==1$;

Peakname = ' B';\%B

IntRange_tau = y; $\%$ integrate within +/- this value

IntRange_t = x; \% integrate within +/- this value

elseif $\mathrm{z}=\mathbf{2}$;

Peakname $={ }^{\prime} A^{\prime} ; \% A$

IntRange_tau = y; $\%$ integrate within +/- this value

IntRange_t $=\mathbf{x} ; \%$ integrate within $+/$ - this value

elseif $\mathrm{z}=\mathbf{=}$ 3;

Peakname $={ }^{\prime}$ A-B';\%A-B

IntRange_tau $=y$; $\%$ integrate within $+/$ - this value

IntRange_t $=\mathbf{x} ; \%$ integrate within $+/$ - this value

elseif $\mathrm{z}=\mathbf{=}$;

Peakname = ' B-A';\%B-A

IntRange_tau =y; $\%$ integrate within +/- this value

IntRange_t $=\mathbf{x}$; $\%$ integrate within $+/$ - this value

end

figlndex $=z+20$;

if strcmp(Peakname, ' $A$ ');

RangeValues $=$ [Hi_energy_pk-IntRange_t Hi_energy_pk+IntRange_t Hi_energy_pk+IntRange_tau -Hi_energy_pk-IntRange_tau]; \% A pop box elseif strcmp(Peakname, ' B');

RangeValues $=$ [Lo_energy_pk-IntRange_t Lo_energy_pk+IntRange_t Lo_energy_pk+IntRange_tau -Lo_energy_pk-IntRange_tau]; \% B pop box 


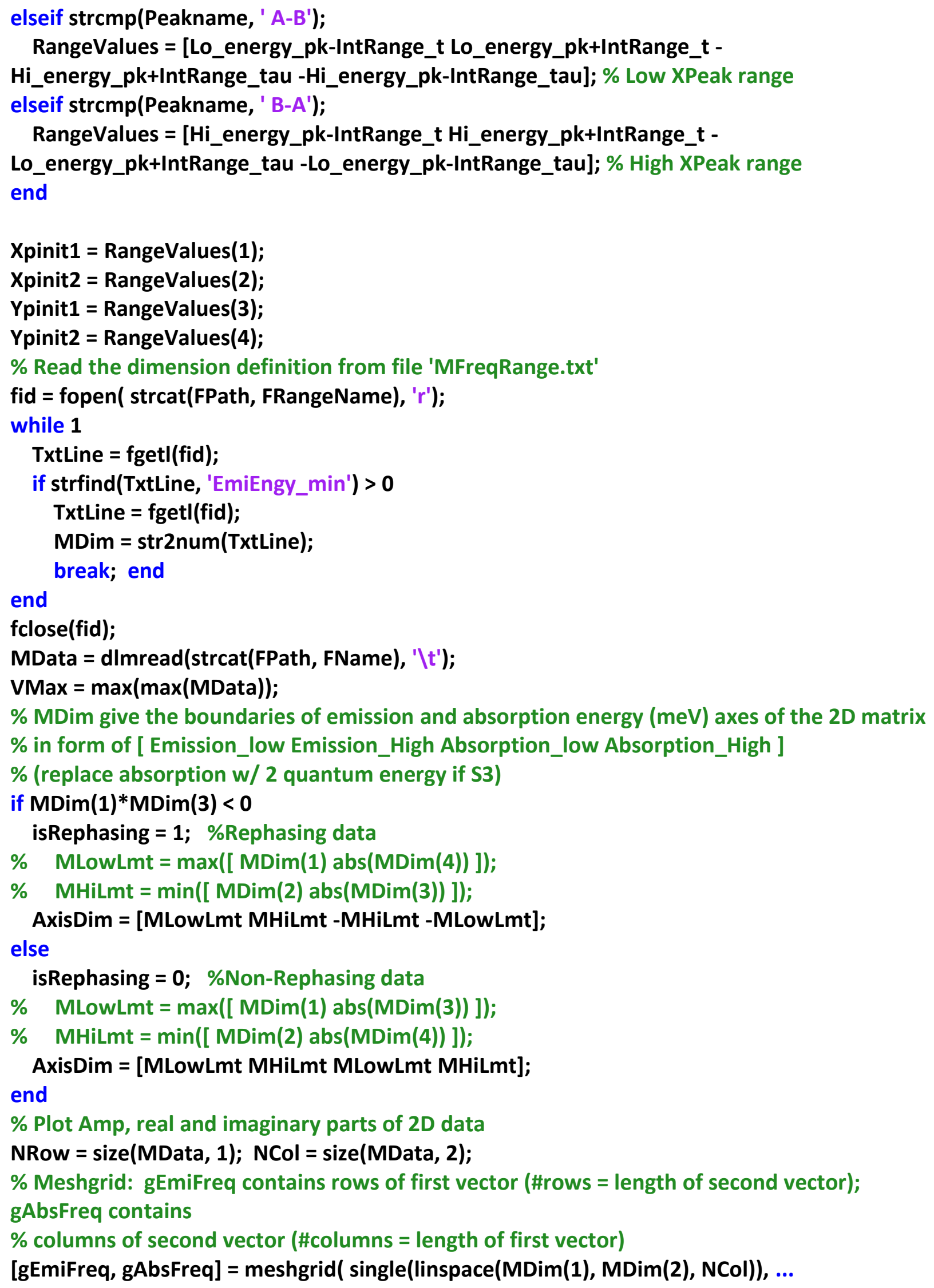


single(linspace(MDim(3), MDim(4), NRow)));

$\% \%$ Plot 2D Amplitude

$\%$ opengl neverselect;

fig1 = figure(1);

set(fig1, 'Position', [ 50200700660 ]);

MData(NRow, NCol-1)= -1; MData(NRow, NCol)=1;

contourf(gEmiFreq, gAbsFreq, MData, linspace(-1, VMax, NContourLevels), 'LineStyle', 'none');

axis(AxisDim);

axis square; colormap jet;

DiagX = linspace( MLowLmt, MHiLmt, LineLength );

DiagY = -DiagX;

\%\% Determine Integration Region

figure(fig1);

datacursormode on;

dcm_obj = datacursormode(fig1);

set(dcm_obj, 'DisplayStyle','window','SnapToDataVertex','off');

$\%$ CurInfo = getCursorInfo(dcm_obj);

$\%[X p Y p]=$ Curlnfo.Position;

$\%$ mouse cursor point at any random position

if $\sim X d$

[Xp, Yp, MouseButton]= ginput(4);

if MouseButton $=1$ break; end

else $X p(1)=X p i n i t 1 ; X p(4)=X p i n i t 1 ; X p(3)=X p i n i t 2 ; X p(2)=X p i n i t 2$;

$Y p(1)=$ Ypinit1; Yp(2) = Ypinit1; Yp(3) = Ypinit2; Yp(4) = Ypinit2;

end

$\% \%$ Calculate and plot lines with values above as corners

htoplength = linspace(Xp(1),Xp(2),LineLength);

hbottomlength = linspace $(X p(4), X p(3)$,LineLength);

vleftlength = linspace(Yp(4),Yp(1),LineLength);

vrightlength = linspace $(Y p(3), Y p(2)$, LineLength $)$;

topslope $=(Y p(2)-Y p(1)) /(X p(2)-X p(1)) ;$

bottomslope $=(Y p(3)-Y p(4)) /(X p(3)-X p(4))$;

leftslope $=(Y p(1)-Y p(4)) /(X p(1)-X p(4))$;

rightslope $=(Y p(3)-Y p(2)) /(X p(3)-X p(2))$;

vtoplength $=Y p(1)+$ topslope. ${ }^{*}$ (htoplength-htoplength(1));

vbottomlength $=Y p(4)+$ bottomslope. ${ }^{*}$ (hbottomlength-hbottomlength(1));

hleftlength $=\mathrm{Xp}(4)+($ vleftlength-vleftlength(1))./leftslope;

hrightlength $=\mathrm{Xp}(3)+($ vrightlength-vrightlength(1))./rightslope;

line(htoplength, vtoplength, 'LineWidth', 2, 'LineStyle', '-', 'Color', [ $\left.\left.\begin{array}{lll}0 & 0 & 0\end{array}\right]\right) ;$

line(hbottomlength, vbottomlength, 'LineWidth', 2, 'LineStyle', '-', 'Color', [ $\left.\left.\begin{array}{lll}0 & 0 & 0\end{array}\right]\right) ;$ 
line(hleftlength, vleftlength, 'LineWidth', 2, 'LineStyle', '-', 'Color', [ $\left.\begin{array}{ll}0 & 0\end{array}\right]$ );

line(hrightlength, vrightlength, 'LineWidth', 2, 'LineStyle', '-', 'Color', [0 0 0]);

$\% \%$ Determine indices in frequency and amplitude data for region within box

$\mathrm{i}=\mathbf{1}$;

while gEmiFreq $(1, \mathrm{i})<\mathrm{Xp}(\mathbf{1})$

$\mathrm{i}=\mathrm{i}+1 ; \%$ lower index for $\mathrm{X}$ integration

end

$\mathrm{j}=\mathbf{1}$;

while gEmiFreq $(1, \mathrm{j})<\mathrm{Xp}(2)$

$\mathbf{j}=\mathbf{j + 1}$; \% upper index for $X$ integration

end

xindex $=\mathrm{j}-\mathrm{i}$;

$k=1$;

while gAbsFreq(k,1) $<$ Yp(4)

$\mathbf{k}=\mathbf{k}+1 ; \%$ lower index for $\mathrm{Y}$ integration

end

I=1;

while gAbsFreq $(1,1)<Y p(1)$

$\mathrm{I}=\mathrm{I}+\mathbf{1}$; \% upper index for $Y$ integration

end

yindex = I-k;

$\% \%$ Meshgrid for region within box

[gEmiFreqInt, gAbsFreqInt] $=$ meshgrid $($ single $($ linspace $(X p(1), X p(2), x i n d e x+1)), \ldots$ single(linspace(Yp(4), Yp(1), yindex+1)));

$\%$ Isolate region of amplitude data within box chosen by indices at

$\%$ beginning and plot

MDatalnt = MData(k:I,i:j);

VMaxInt $=\max (\max ($ MDatalnt $))$;

figure(figIndex)

MData(NRow, NCol-1)= -1; MData(NRow, NCol)= 1;

contourf(gEmiFreqInt, gAbsFreqInt, MDataInt, linspace(0, VMaxInt, NContourLevels), 'LineStyle', 'none');

$\operatorname{axis([Xp(1)Xp(2)Yp(4)Yp(1)]);~}$

axis square; colormap jet;

$\% \%$ Determine average line integral value around integration region box

$\%$ use this ave. line int. value as the average value to subtract from all

$\%$ the plots.

bottom = sum(MData(k,i:j))/(xindex+1);

top $=\operatorname{sum}(M \operatorname{Mata}(\mathrm{l}, \mathrm{i}: \mathrm{j})) /(\mathrm{xindex}+1)$;

left = sum(MData(k:l,i))/(yindex+1);

right $=\operatorname{sum}(\operatorname{MData}(k: I, j)) /($ yindex+1);

$\% \%$ Find position of $\max$ 


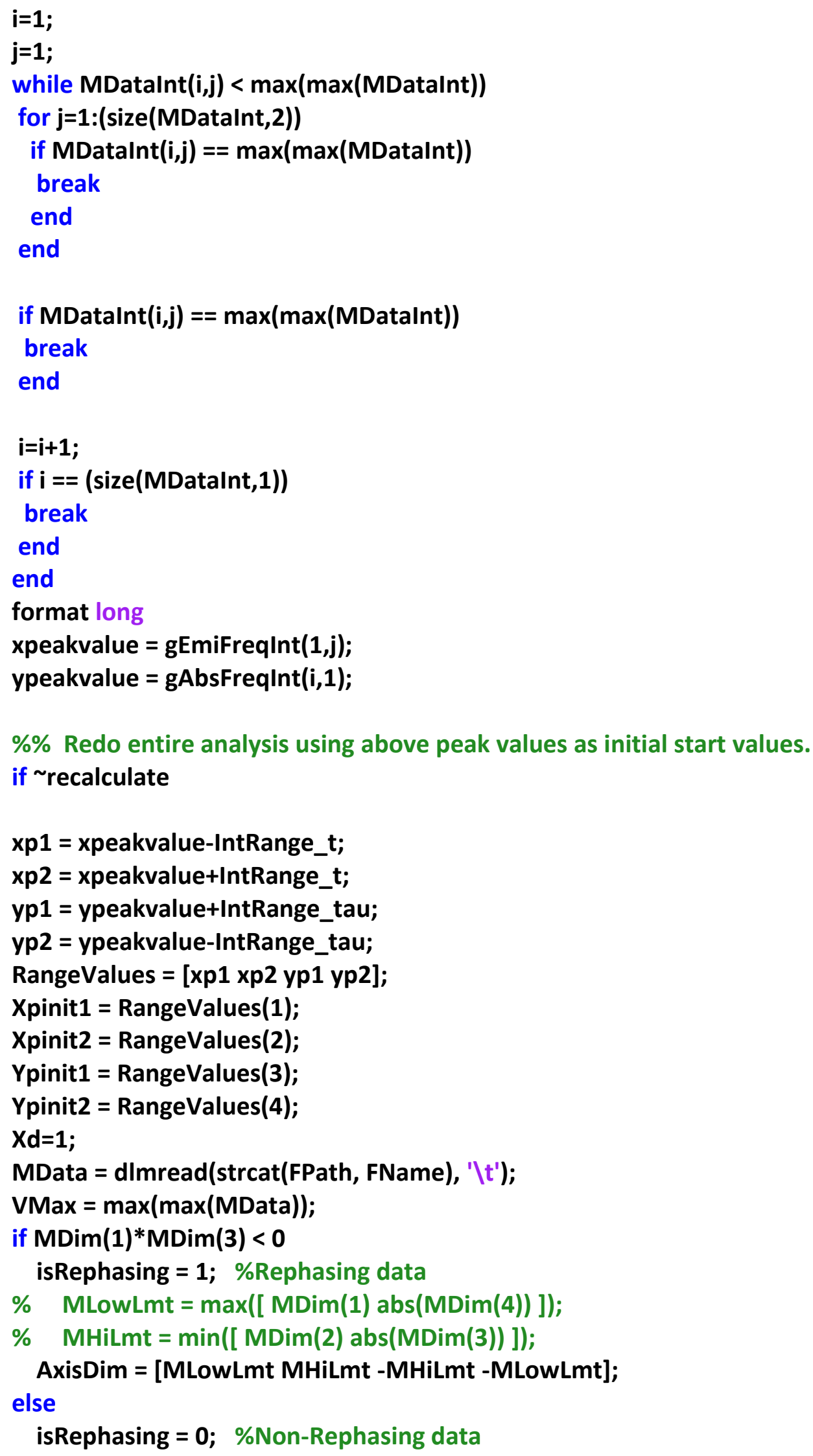




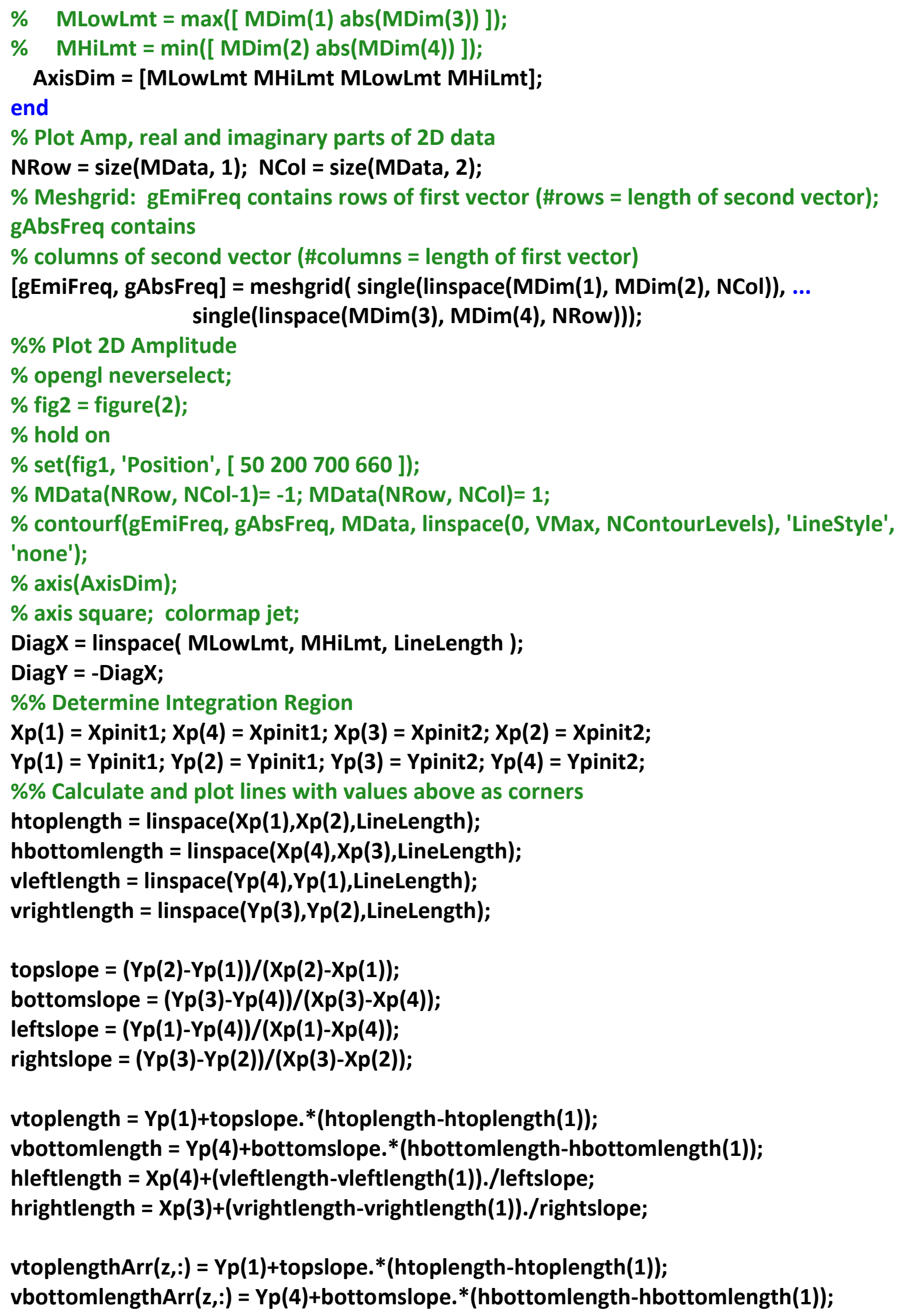


hleftlengthArr(z,:) =Xp(4)+(vleftlength-vleftlength(1))./leftslope;

hrightlength $\operatorname{Arr}(z,:)=X p(3)+($ vrightlength-vrightlength(1))./rightslope;

htoplengthArr(z,:) = linspace $(X p(1), X p(2)$,LineLength);

hbottomlengthArr(z,:) = linspace $(X p(4), X p(3)$, LineLength);

vleftlengthArr(z,:) = linspace(Yp(4),Yp(1),LineLength);

vrightlengthArr( $(2,:)=$ linspace(Yp(3),Yp(2),LineLength);

line(htoplength, vtoplength, 'LineWidth', 2, 'LineStyle', '-', 'Color', [ $\left.\begin{array}{ll}0 & 0\end{array}\right]$ );

line(hbottomlength, vbottomlength, 'LineWidth', 2, 'LineStyle', '-', 'Color', [ $\left.\left.\begin{array}{lll}0 & 0 & 0\end{array}\right]\right) ;$

line(hleftlength, vleftlength, 'LineWidth', 2, 'LineStyle', '-', 'Color', [ $\left.\left.\begin{array}{lll}0 & 0 & 0\end{array}\right]\right) ;$

line(hrightlength, vrightlength, 'LineWidth', 2, 'LineStyle', '-', 'Color', [ $\left.\left[\begin{array}{lll}0 & 0 & 0\end{array}\right]\right) ;$

$\% \%$ Determine indices in frequency and amplitude data for region within box

i=1;

while gEmiFreq(1,i) $<\mathrm{Xp}(\mathbf{1})$

$\mathbf{i}=\mathbf{i}+\mathbf{1}$; \% lower index for $\mathrm{X}$ integration

end

$\mathrm{j}=1$;

while gEmiFreq $(1, j)<X p(2)$

$\mathrm{j}=\mathrm{j}+1$; \% upper index for $\mathrm{X}$ integration

end

xindex $=\mathrm{j}-\mathrm{i}$;

$k=1$;

while gAbsFreq(k,1) $<\mathrm{Yp}(4)$

$\mathbf{k}=\mathbf{k}+1 ; \%$ lower index for $Y$ integration

end

I=1;

while gAbsFreq $(\mathrm{I}, 1)<\mathrm{Yp}(1)$

$I=I+1 ; \%$ upper index for $Y$ integration

end

yindex = I-k;

$\% \%$ Meshgrid for region within box

[gEmiFreqInt, gAbsFreqInt] $=$ meshgrid $(\operatorname{single}(\operatorname{linspace}(\mathrm{Xp}(1), \mathrm{Xp}(2)$, xindex+1)), ... single(linspace(Yp(4), Yp(1), yindex+1)));

$\%$ Isolate region of amplitude data within box chosen by indices at

$\%$ beginning and plot

MDataInt = MData(k:I,i:j);

VMaxInt $=\max (\max ($ MDataInt $))$;

figure(figlndex)

MData(NRow, NCol-1)= -1; MData(NRow, NCol)=1;

contourf(gEmiFreqInt, gAbsFreqInt, MDatalnt, linspace(0, VMaxInt, NContourLevels), 'LineStyle', 'none');

title(Peakname);

$\operatorname{axis([Xp(1)Xp(2)Yp(4)Yp(1)]);~}$

axis square; colormap jet; 
\%\% Determine average line integral value around integration region box $\%$ use this avg line integral value as the average value to subtract from all $\%$ the plots. Bottom, top,... refer to sides of the integration region box bottom = sum(MData $(k, i: j)) /(x i n d e x+1)$;

top $=\operatorname{sum}(\mathrm{MData}(\mathrm{l}, \mathrm{i}: \mathrm{j})) /(\mathrm{xindex}+1)$;

left = sum(MData(k:I,i))/(yindex+1);

right = sum(MData(k:I,j))/(yindex+1);

Bkgd $=($ bottom + top + left + right $) / 4$

$\% \%$ Sum all amplitude values within this region (Integration average part) IntegrationAverage = sum(sum(MDatalnt) $) /(\operatorname{size}($ MDatalnt,1)*size $($ MDatalnt,2) $)$;

IntegrationTotal = sum(sum(MDatalnt));

$\% \%$ Find max value and position

Max $=\max (\max ($ MDatalnt $)) ; \%$ maximum of integration region

$\mathrm{i}=1$;

$\mathrm{j}=1$;

while MDatalnt $(i, j)<\max (\max ($ MDatalnt $))$

for $\mathrm{j}=\mathbf{1}$ :(size(MDatalnt,2))

if $M D a t a I n t(i, j)==\max (\max ($ MDataInt $))$

break

end

end

if MDatalnt $(i, j)==\max (\max ($ MDatalnt $))$

break

end

$\mathrm{i}=\mathrm{i}+1$;

if $i==($ size(MDatalnt,1))

break

end

end

format long

xpeakvalue $=$ gEmiFreqInt $(1, \mathrm{j})$;

G = transpose(gAbsFreqInt);

ypeakvalue = gAbsFreqInt $(i, 1)$;

else

Bkgd $=($ bottom + top + left + right $) / 4 ; \%$ average line integral around the peak's box IntegrationAverage $=\operatorname{sum}(\operatorname{sum}($ MDatalnt $)) /(\operatorname{size}($ MDatalnt,1)*size $($ MDatalnt,2));

IntegrationTotal = sum(sum(MDataInt));

Max $=\max (\max ($ MDatalnt $))$;

xpeakvalue $=$ gEmiFreqInt $(1, \mathrm{j})$;

ypeakvalue = gAbsFreqInt(i,1);

end

vtoplength $=$ Yp(1)+topslope. ${ }^{*}$ (htoplength-htoplength(1)); 
vbottomlength $=Y p(4)+$ bottomslope. ${ }^{*}($ hbottomlength-hbottomlength(1));

hleftlength $=X p(4)+(v$ leftlength-vleftlength(1))./leftslope;

hrightlength $=\mathrm{Xp}(3)+($ vrightlength-vrightlength(1))./rightslope;

vtoplengthArr(z,:) = Yp(1)+topslope.*(htoplength-htoplength(1));

vbottomlengthArr(z,:) = Yp(4)+bottomslope.*(hbottomlength-hbottomlength(1));

hleftlengthArr(z,:) = Xp(4)+(vleftlength-vleftlength(1))./leftslope;

hrightlengthArr(z,:) =Xp(3)+(vrightlength-vrightlength(1))./rightslope;

htoplengthArr(z,:) = linspace(Xp(1),Xp(2),LineLength);

hbottomlengthArr(z,:) = linspace $(X p(4), X p(3)$, LineLength);

vleftlengthArr(z,:) = linspace(Yp(4),Yp(1), LineLength);

vrightlengthArr(z,:) = linspace(Yp(3),Yp(2),LineLength);

line(htoplength, vtoplength, 'LineWidth', 2, 'LineStyle', '-', 'Color', [ $\left.\left.\begin{array}{lll}0 & 0 & 0\end{array}\right]\right) ;$

line(hbottomlength, vbottomlength, 'LineWidth', 2, 'LineStyle', '-', 'Color', [ $\left.\left.\begin{array}{lll}0 & 0 & 0\end{array}\right]\right) ;$

line(hleftlength, vleftlength, 'LineWidth', 2, 'LineStyle', '-', 'Color', [ $\left.\left.\begin{array}{lll}0 & 0 & 0\end{array}\right]\right) ;$

line(hrightlength, vrightlength, 'LineWidth', 2, 'LineStyle', '-', 'Color', [ $\left.\begin{array}{ll}0 & 0\end{array}\right]$ );

format long

values = [xpeakvalue ypeakvalue Max IntegrationAverage IntegrationTotal Bkgd];

dlmwrite(strcat(FOutPath,FFIndex, Peakname,' values.dat'),values, 'delimiter','\t','precision',

6);

$z=z+1$;

end

figure (3)

MData(NRow, NCol-1)= -1; MData(NRow, NCol)= 1;

contourf(gEmiFreq, gAbsFreq, MData, linspace(0, VMax, NContourLevels), 'LineStyle', 'none'); axis(AxisDim);

title(strcat('Scan 2D',num2str(m)));

axis square; colormap jet;

for $n=1: 4$

hold on

line(htoplengthArr(n,:), vtoplengthArr(n,:), 'LineWidth', 2, 'LineStyle', '-', 'Color', [0 00 ]);

line(hbottomlengthArr(n,:), vbottomlengthArr(n,:), 'LineWidth', 2, 'LineStyle', '-', 'Color', [0

0 0]);

line(hleftlengthArr(n,:), vleftlengthArr(n,:), 'LineWidth', 2, 'LineStyle', '-', 'Color', [0 0 0]); line(hrightlengthArr(n,:), vrightlengthArr(n,:), 'LineWidth', 2, 'LineStyle', '-', 'Color', [0 00 0 $]$ ); hold off

end

saveas(gcf,strcat(FOutPath,FFIndex, ' Integration Boxes'), 'bmp');

$\% \%$ Determine if boxes overlap

$A=[$ hbottomlengthArr(1,1) vleftlengthArr(1,1) $\max ($ hbottomlengthArr(1,:))-

min(hbottomlengthArr(1,:))...

$\max (\operatorname{abs}($ vrightlengthArr(1,:)))-min(abs(vrightlengthArr(1,:)))]; 
$B=[$ hbottomlengthArr $(2,1)$ vleftlengthArr $(2,1) \max ($ hbottomlengthArr $(2,:))-$

min(hbottomlengthArr(2,:))...

$\max ($ abs(vrightlengthArr(2,:)))-min(abs(vrightlengthArr(2,:)))];

$\mathrm{C}=[$ hbottomlengthArr $(3,1)$ vleftlengthArr $(3,1) \max ($ hbottomlengthArr $(3,:))$ -

min(hbottomlengthArr(3,:))...

max(abs(vrightlengthArr(3,:)))-min(abs(vrightlengthArr(3,:)))];

$D=[$ hbottomlengthArr $(4,1)$ vleftlengthArr $(4,1) \max ($ hbottomlengthArr(4,:))-

min(hbottomlengthArr(4,:))...

$\max ($ abs(vrightlengthArr(4,:)))-min(abs(vrightlengthArr(4,:)))];

if $\operatorname{rectint}(A, B)|\operatorname{rectint}(A, C)| \operatorname{rectint}(A, D)|\operatorname{rectint}(B, C)| \operatorname{rectint}(B, D) \mid \operatorname{rectint}(C, D)$

strcat('Overlap on scan_',num2str(m))

break

end

end

A.17 Neutral Density Filter Correction Code (ND_Adj.m)

\%Adjusts FWM initial trace, TI FWM vs tau, and integrated peaks for ND

\%filters. It subtracts the background from FWM, and integrated $2 \mathrm{~d}$ peaks. If

$\%$ no ND filter was used it just copies the files over, less the backgrounds.

$\% \%$ Set directory for files and filenames

clear all; clc; close all

FIndexStart $=1 ; \%$ Specify start scan index

FIndexEnd $=56$; $\%$ Specify last scan index

Background = dlmread(strcat('.l','Bkgd01.dat'), '\t');

for $m=$ FIndexStart:FIndexEnd;

FFIndex = num2str(m);

RootDir = strcat('.','\2D',FFIndex, '\');

OutputDir = '.|ND Adjusted \';

\%Make target directory for each ND adjusted traces

if exist('. \ND Adjusted')

mkdir('.I','ND Adjusted');

end

$\% \%$ Read in raw data and subtract background

$\% \quad F W M=$ dlmread(strcat(RootDir,'FWM',FFIndex,'.dat'), '\t');

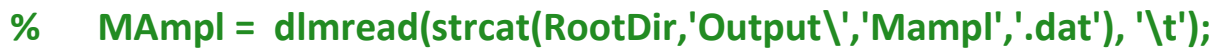

$\% \quad$ RtrFWMvsTau = dlmread(strcat(RootDir,'Output $\mid$ ','RtrFWMvsTau','.dat'), '\t');

$\% \quad$ B_Int_Pk = dlmread(strcat('. Integrated Datal',FFIndex,' B values.dat'), '\t');

$\% \quad$ BA_Int_Pk= dlmread(strcat('..Integrated Datal',FFIndex,' B-A values.dat'), '\t');

$\% \quad$ A_Int_Pk = dlmread(strcat('. Integrated Datal',FFIndex,' A values.dat'), ' 'It');

$\% \quad A B \_I n t \_P k=d l m r e a d(s t r c a t(' . \mid$ Integrated Datal',FFIndex,' A-B values.dat'), '\t'); 
if exist(strcat('. Integrated Data \',FFIndex,' Biexciton values.dat'))

Biexciton_Pk = dlmread(strcat('.।Integrated Data\',FFIndex,' Biexciton values.dat'), '\t');

else end

$\% \%$ Calculate ND

startpixel = 350;

endpixel = 724;

$\%$ startpixel $=1$;

$\%$ endpixel = 1023;

\%Read in and calculate the OD of the spectrometer filter

if exist(strcat(RootDir,'ND_Incident',FFIndex,'.dat'),'file')

ND_Incident= dlmread(strcat(RootDir,'ND_Incident',FFIndex,'.dat'), '\t');

ND_Incident1= ND_Incident(startpixel:endpixel,2)-sum(Background(:,2))/1024;

\%ND_Incident1= ND_Incident(:,2)-sum([ND_Incident(1:10,2);

ND_Incident(1015:end,2)])/20;

ND_Trans = dlmread(strcat(RootDir,'ND_Trans',FFIndex,'.dat'), '\t');

ND_Trans1= ND_Trans(startpixel:endpixel,2)-sum(Background(:,2))/1024;

\%ND_Trans1= ND_Trans(:,2)-sum([ND_Trans(1:10,2); ND_Trans(1015:end,2)])/20;

ND $=\log 10($ trapz(ND_Incident1/trapz(ND_Trans1)))

$\% \quad$ ND2 $=\log 10(($ ND_Incident1./(ND_Trans1) $))$;

end

\%Read in and calculate the OD of the FWM only filter

if exist(strcat(RootDir,'FWM_ND_Incident',FFIndex,'.dat'),'file')

FWM_ND_Incident= dlmread(strcat(RootDir,'FWM_ND_Incident',FFIndex,' .dat'), '\t');

FWM_ND_Incident1=FWM_ND_Incident(startpixel:endpixel,2)-

sum(Background(:,2))/1024;

\%ND_Incident1= ND_Incident(:,2)-sum([ND_Incident(1:10,2);

ND_Incident(1015:end,2)])/20;

FWM_ND_Trans = dlmread(strcat(RootDir,'FWM_ND_Trans',FFIndex, '.dat'), '\t');

FWM_ND_Trans1=FWM_ND_Trans(startpixel:endpixel,2)-sum(Background(:,2))/1024;

\%ND_Trans1=ND_Trans(:,2)-sum([ND_Trans(1:10,2); ND_Trans(1015:end,2)])/20;

FWM_ND = log10(trapz(FWM_ND_Incident1/trapz(FWM_ND_Trans1)))

else

FWM_ND = 0;

end

$\%$ figure(1)

$\%$ hold on

$\%$ plot(ND_Trans(startpixel:endpixel,1),ND2)

$\%$ plot(ND_Trans(startpixel:endpixel,1),FWM_ND_Trans1,'g')

$\%$ hold off

$\%$ title(FFIndex) 
$\%$ ND2 = log10(ND_Incident1./ND_Trans1);

$\% \%$

$\%$ figure(1)

$\%$

$\%$ plot(ND_Trans(startpixel:endpixel,1),FWM_ND)

$\%$ title(FFIndex)

$\% \%$ Correct Spectra for ND filters, if ND filter was present subtract background from

integrated peaks

if exist(strcat(RootDir, 'ND_Incident',FFIndex, '.dat'),'file')

$\%$ FWM_Adj(:,2)=(FWM(:,2)-

sum(Background(:,2))/1024)*10^(ND+FWM_ND);FWM_Adj(:,1)=FWM(:,1);

$\%$ Mampl_Adj=MAmpl*10^(ND+FWM_ND );

$\% \%$

RtrFWMvsTau_Adj(:,2)=RtrFWMvsTau(:,2)*10^(ND);RtrFWMvsTau_Adj(:,1)=RtrFWMvsTau(:,1

);

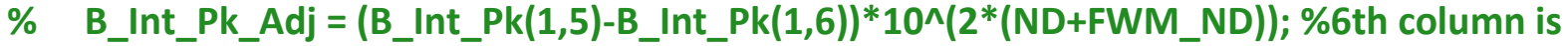
background

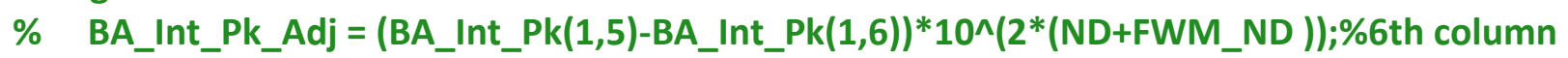
is background

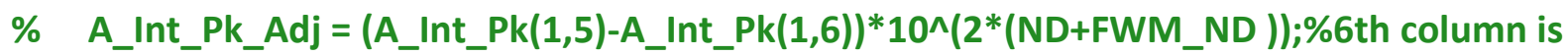
background

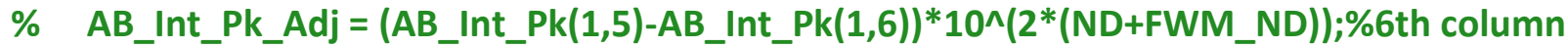
is background

if exist(strcat('. Integrated Data\',FFIndex,' Biexciton values.dat'))

Biexciton_Int_Pk_Adj $=($ Biexciton_Pk(1,5)-Biexciton_Pk $(1,6)) * 10^{\wedge}\left(2^{*}(\right.$ ND+FWM_ND $\left.)\right) ; \% 6$ th column is background else end

dImwrite(strcat(OutputDir,FFIndex,' Biexciton values NDadj.dat'),Biexciton_Int_Pk_Adj);

\% dlmwrite(strcat(OutputDir,'FWM',FFIndex,'.dat'), FWM_Adj);

\% dlmwrite(strcat(OutputDir,'Mampl',FFIndex,'.dat'), Mampl_Adj);

\% dlmwrite(strcat(OutputDir,'RtrFWMvsTau','.dat'), RtrFWMvsTau_Adj);

$\%$ dlmwrite(strcat(OutputDir,FFIndex,' B values NDadj.dat'), B_Int_Pk_Adj);

$\%$ dlmwrite(strcat(OutputDir,FFIndex,' B-A values NDadj.dat'),BA_Int_Pk_Adj);

$\%$ dlmwrite(strcat(OutputDir,FFIndex,' A values NDadj.dat'),A_Int_Pk_Adj);

\% dlmwrite(strcat(OutputDir,FFIndex,' A-B values NDadj.dat'),AB_Int_Pk_Adj);

else

$\%$ error $=$ strcat('no nd files ',FFIndex)

$\% \quad \mathrm{ND}=0$;

$\%$ FWM_Adj(:,2)=(FWM(:,2)-

$\operatorname{sum}($ Background(:,2))/1024)*10^(ND+FWM_ND);FWM_Adj(:,1)=FWM(:,1);

$\%$ Mampl_Adj=MAmpl*10^(ND+FWM_ND ); 
$\% \%$

RtrFWMvsTau_Adj(:,2)=RtrFWMvsTau(:,2)*10^(ND);RtrFWMvsTau_Adj(:,1)=RtrFWMvsTau(:,1

);

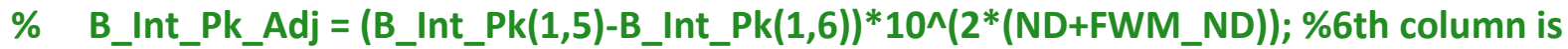
background

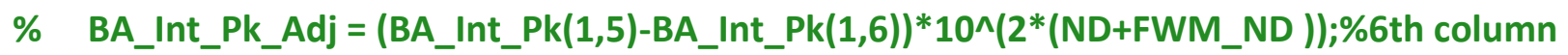
is background

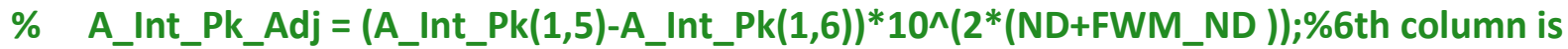
background

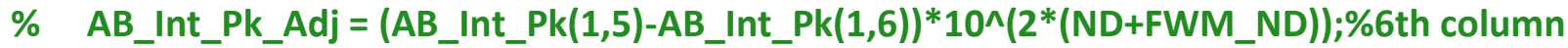
is background

$\%$

\% dlmwrite(strcat(OutputDir,'FWM',FFIndex,'.dat'), FWM_Adj);

$\%$ dlmwrite(strcat(OutputDir,'Mampl',FFIndex,'.dat'), Mampl_Adj);

\% \% dlmwrite(strcat(OutputDir,'RtrFWMvsTau','.dat'), RtrFWMvsTau_Adj);

\% dlmwrite(strcat(OutputDir,FFIndex,' B values NDadj.dat'), B_Int_Pk_Adj);

\% dlmwrite(strcat(OutputDir,FFIndex,' B-A values NDadj.dat'),BA_Int_Pk_Adj);

\% dlmwrite(strcat(OutputDir,FFIndex,' A values NDadj.dat'),A_Int_Pk_Adj);

$\%$ dlmwrite(strcat(OutputDir,FFIndex,' A-B values NDadj.dat'),AB_Int_Pk_Adj);

end

$\%$ figure(2)

$\%$ hold on

$\%$ plot(FWM_Adj(:,1),FWM_Adj(:,2));

$\%$ plot(FWM(:,1),FWM(:,2),'-r');

$\%$ hold off

$\% \%$

\% clear FWM_Adj Mampl RtrFWMvsTau_Adj B_Int_Pk1 BA_Int_Pk1 A_Int_Pk1 AB_Int_Pk1 end

\section{A.18 Collect Individual Data Files for Large Strain Data Set (aggregator.m)}

\%Aggregates data from multiple scans into matricies

clear all; clc; close all

FIndexStart $=1 ; \%$ Specify start scan index $* *$ MUST START AT 1 !**

FIndexEnd=100;\%Specify last scan index

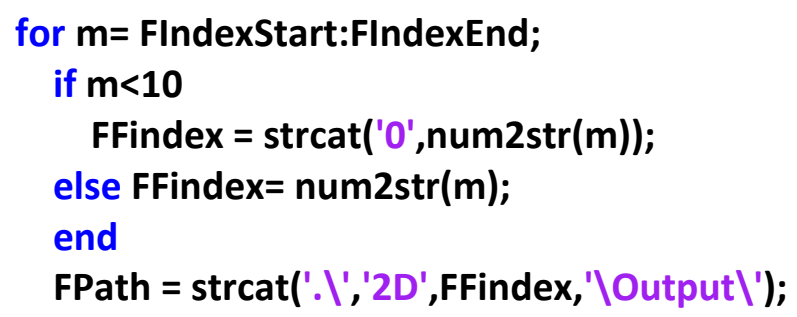




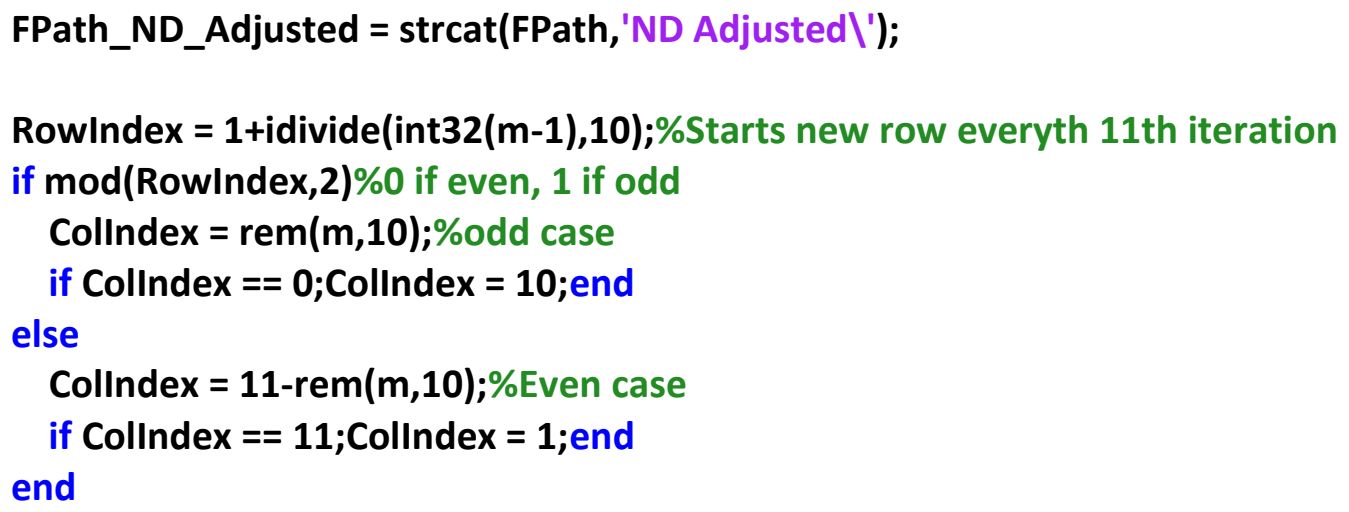

AdiagWidths(RowIndex,Collndex) = abs $($ InputA(1,3));

AdiagResidual(RowIndex,Collndex) = InputA(1,5);

ACrsdiagWidths(RowIndex,Collndex $)=$ abs $($ InputA(1,8));

ACrsResidual(RowIndex,Collndex) = InputA(1,10);

AEmEnergy(RowIndex,Collndex) = Input $A(1,12)$;

BdiagWidths(Rowlndex,Collndex $)=$ abs (InputB(1,3));

BdiagResidual (RowIndex,Collndex) = InputB(1,5);

BCrsdiagWidths (RowIndex,Collndex $)=$ abs $($ InputB $(1,8))$;

BCrsResidual(RowIndex,Collndex) = InputB $(1,10)$;

BEmEnergy(RowIndex,Collndex) = InputB(1,12);

\%\% Read in data from integrated 2D peaks

\%A B-A Peak positions in 2D

$\% A-B \quad B$

FNameB2 = 'B_Int_Pk.dat';

FNameBA2 = 'BA_Int_Pk.dat';

FNameA2 = 'A_Int_Pk.dat';

FNameAB2 = 'AB_Int_Pk.dat';

A_Integrated(RowIndex,Collndex) = dlmread(strcat(FPath_ND_Adjusted,FNameA2)); AB_Integrated(RowIndex,Collndex) = dlmread(strcat(FPath_ND_Adjusted,FNameAB2)); B_Integrated(RowIndex,Collndex) = dlmread(strcat(FPath_ND_Adjusted,FNameB2)); BA_Integrated(RowIndex,Collndex) = dlmread(strcat(FPath_ND_Adjusted,FNameBA2)); 
end

Sum_Int_spec $=$ A_Integrated+B_Integrated+AB_Integrated+BA_Integrated;

PowerAxis = [.025 .05 .1 .15 .2 .3 . . .9 1.1]; \%mW

TempAxis = [6 810121417202530 40]; \%Kelvin

$\% \%$ Save all the compiled data to dat files

dlmwrite(strcat('.।','A Diag Withs','.dat'), AdiagWidths, '\t');

dlmwrite(strcat('.l','B Diag Withs','.dat'), BdiagWidths,' $\backslash t$ ');

dlmwrite(strcat('.।','A Diag Residuals','.dat'), AdiagResidual,'ไt');

dlmwrite(strcat('.।','B Diag Residuals','.dat'), BdiagResidual,' \t');

dlmwrite(strcat('.।','A Cross Diag Withs','.dat'), ACrsdiagWidths,' \t');

dlmwrite(strcat('.।','B Cross Diag Withs','.dat'), BCrsdiagWidths, '\t');

dlmwrite(strcat('.।','A Cross Diag Residuals','.dat'), ACrsResidual,' \t');

dlmwrite(strcat('.।','B Cross Diag Residuals','.dat'), BCrsResidual,' \t');

dlmwrite(strcat('.l','A Center Emis Energys','.dat'), AEmEnergy, '\t');

dlmwrite(strcat('.।','B Center Emis Energys','.dat'), BEmEnergy, '\t');

dlmwrite(strcat('. I','A 2D Integreated Peaks','.dat'), A_Integrated, '\t');

dlmwrite(strcat('. I','B 2D Integreated Peaks','.dat'), B_Integrated,' '\t');

dlmwrite(strcat('. I','AB 2D Integreated Peaks','.dat'), AB_Integrated,' \t');

dlmwrite(strcat('.l','BA 2D Integreated Peaks','.dat'), BA_Integrated,' \t');

dlmwrite(strcat('.।','Sum 2D Integreated Peaks','.dat'), Sum_Int_spec,'ไt');

dlmwrite(strcat('.।','Power Axis K','.dat'), PowerAxis','\t');

dlmwrite(strcat('.।','Temp Axis mW','.dat'), TempAxis','\t');

\section{A.19 Render Various Plots from Strain Data Set (aggplotter.m)}

$\% \%$ Plots slices of aggregated whole data set

clear all; clc; close all

$\%$ Read in all the data files

Sum_Int_spec = dImread('.|Sum 2D Integreated Peaks.dat','’t');

BA_Integrated = dlmread('.\BA 2D Integreated Peaks.dat',' \t');

AB_Integrated = dlmread('. \AB 2D Integreated Peaks.dat',' $\backslash t$ ');

B_Integrated = dlmread('.|B 2D Integreated Peaks.dat',' \t');

A_Integrated = dlmread('.\A 2D Integreated Peaks.dat',' \t');

BEmEnergy = dlmread('.।B Center Emis Energys.dat','\t');

AEmEnergy = dlmread('.\A Center Emis Energys.dat','\t');

BCrsResidual = dlmread('. \B Cross Diag Residuals.dat','\t');

ACrsResidual = dlmread('. \A Cross Diag Residuals.dat',' $\backslash t ')$;

BCrsdiagWidths = dlmread('. \B Cross Diag Withs.dat','\t');

ACrsdiagWidths = dlmread('. \A Cross Diag Withs.dat',' $\backslash t$ ');

BdiagResidual = dlmread('.|B Diag Residuals.dat',' \t');

AdiagResidual = dlmread('. \A Diag Residuals.dat','\t'); 


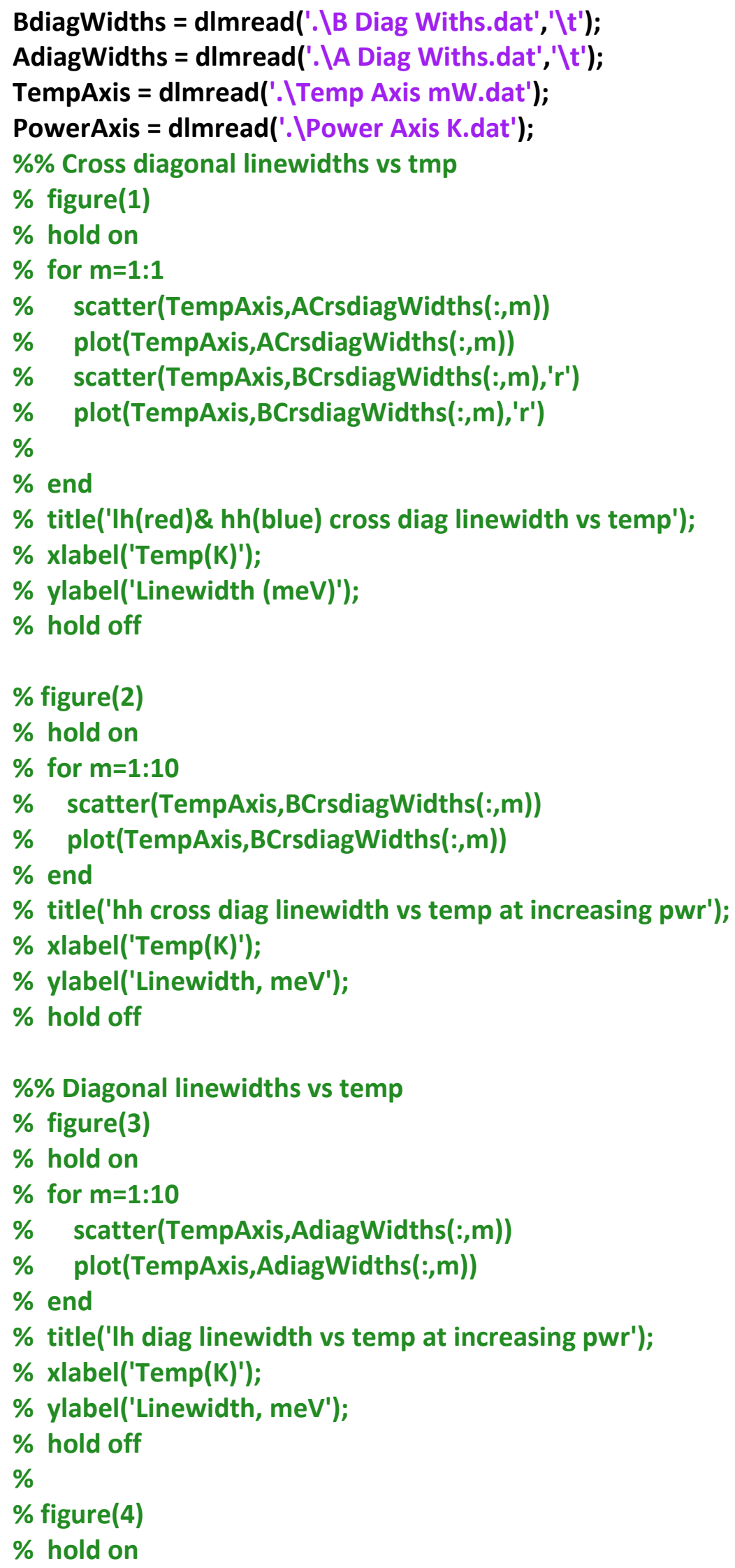


$\%$ for $m=1: 10$

\% scatter(TempAxis,BdiagWidths(:,m))

$\%$ plot(TempAxis,BdiagWidths(:,m))

$\%$ end

$\%$ title('hh diag linewidth vs temp at increasing pwr');

$\%$ xlabel('Temp(K)');

$\%$ ylabel('Linewidth, meV');

$\%$ hold off

$\% \%$ Diagonal linewidths vs power

$\%$ figure(5)

$\%$ hold on

$\%$ for $m=1: 10$

$\% \quad$ scatter(PowerAxis,AdiagWidths $(m,:))$

$\% \quad$ plot(PowerAxis,AdiagWidths(m,:))

$\%$ end

$\%$ title('Ih diag linewidth vs power at increasing temp');

\% xlabel('Power(mW)');

\% ylabel('Linewidth, meV');

$\%$ hold off

$\%$

$\%$ figure(6)

$\%$ hold on

$\%$ for $m=1: 10$

\% scatter(PowerAxis,BdiagWidths(m,:))

$\%$ plot(PowerAxis,BdiagWidths $(m,:))$

$\%$ end

$\%$ title('hh diag linewidth vs power at increasing temp');

$\%$ xlabel('Power(mW)');

$\%$ ylabel('Linewidth, meV');

$\%$ hold off

$\% \%$ Cross diagonal linewidths vs power

$\%$ figure(7)

$\%$ hold on

$\%$ for $m=1: 1$

\% scatter(PowerAxis,ACrsdiagWidths $(m,:))$

$\% \quad \% p l o t(P o w e r A x i s, A C r s d i a g W i d t h s(m,:))$

$\%$ scatter(PowerAxis,BCrsdiagWidths(m,: $),{ }^{\prime} r$ ')

$\% \%$ plot(PowerAxis,BCrsdiagWidths(m,:),'r')

$\% x \lim \left(\left[\begin{array}{ll}0 & 1.2\end{array}\right]\right)$

\% errorbar(PowerAxis,BCrsdiagWidths(m,:),BCrsResidual(m,:),'r')

$\%$ errorbar(PowerAxis,ACrsdiagWidths(m,:),BCrsResidual $(m,:))$

$\%$ end

$\%$ title('Ih(blue) \& hh(red) cross diag linewidth vs Power at 6 K');

$\%$ xlabel('Power (mW)'); 
\% ylabel('Linewidth, meV');

$\%$ hold off

$\%$

$\%$ figure(8)

$\%$ hold on

$\%$ for $m=1: 10$

\% scatter(PowerAxis,BCrsdiagWidths(m,:))

$\%$ plot(PowerAxis,BCrsdiagWidths $(m,:))$

$\%$ end

$\%$ title('hh cross diag linewidth vs Power at increasing Temp');

$\%$ xlabel('Power (mW)');

$\%$ ylabel('Linewidth, meV');

$\%$ hold off

\%\% Peak A diagonal fit residuals

$\%$ figure(9)

$\%$ hold on

$\%$ for $m=1: 10$

$\%$ scatter(TempAxis,AdiagResidual(:,m))

$\%$ plot(TempAxis,AdiagResidual(:,m))

$\%$ end

$\%$ title('Ih cross diag residuals vs temp at increasing power');

$\%$ xlabel('Power (mW)');

$\%$ ylabel('Residual, meV');

$\%$ hold off

\%\% Integrate peak A vs power and temp \%\%\%\%\%\%\%\%\%\%\%\%\%\%\%\%\%\%\%\%\%\%\%

$\%$ figure(10)

$\%$

$\%$ for $m=1: 10$

\% \%scatter(PowerAxis,log10(A_Integrated(m,:)))

$\%$ semilogy(PowerAxis, $A$ _Integrated $(m,:))$

$\%$ hold on

$\%$ end

$\%$ title('Peak "A" integrated vs Power at increasing Temp');

$\%$ xlabel('Power (mW)');

$\%$ ylabel('Integrated intensity');

$\%$ hold off

$\%$ figure(11)

$\%$ hold on 
$\%$ for $m=1: 10$

\% scatter(TempAxis,log10(A_Integrated(:,m)))

$\%$ plot(TempAxis,log10(A_Integrated(:,m)))

$\%$ end

$\%$ title('Peak "A" integrated vs Temp at increasing pwr');

$\%$ xlabel('Temp(k)');

$\%$ ylabel('Integrated intensity');

$\%$ hold off

$\% \%$ Integrate peak B vs power and temp \%\%\%\%\%\%\%\%\%\%\%\%\%\%\%\%\%\%\%\%\%\%\%

$\%$ figure(12)

$\%$ hold on

$\%$ for $m=1: 10$

\% scatter(PowerAxis,log10(B_Integrated(m,:)))

$\%$ plot(PowerAxis,log10(B_Integrated $(\mathrm{m},:)))$

$\%$

$\%$ end

$\%$ title('Peak "B" integrated vs Power at increasing Temp');

$\%$ xlabel('Power (mW)');

$\%$ ylabel('Integrated intensity');

$\%$ hold off

$\%$

$\%$ figure(13)

$\%$ hold on

$\%$ for $m=1: 10$

$\%$ scatter(TempAxis,log10(B_Integrated(:,m)))

$\%$ plot(TempAxis,log10(B_Integrated(:,m)))

$\%$ end

$\%$ title('Peak "B" integrated vs Temp at increasing pwr');

$\%$ xlabel('Temp(k)');

$\%$ ylabel('Integrated intensity');

$\%$ hold off

$\% \%$ Integrated peak A-B vs power and temp \%\%\%\%\%\%\%\%\%\%\%\%\%\%\%\%\%\%\%\%\%\%\%

$\%$ figure(14)

$\%$ hold on

$\%$ for $m=1: 10$

\% scatter(PowerAxis,log10(AB_Integrated $(\mathrm{m},:)))$

$\%$ plot(PowerAxis,log10(AB_Integrated(m,: $))$ )

$\%$ end

$\%$ title('Peak "A-B" integrated vs Power at increasing Temp');

$\%$ xlabel('Power (mW)');

$\%$ ylabel('Integrated intensity');

$\%$ hold off

$\%$

$\%$ figure(15) 
$\%$ hold on

$\%$ for $m=1: 10$

$\%$ scatter(TempAxis,log10(AB_Integrated(:,m)))

$\%$ plot(TempAxis,log10(AB_Integrated(:,m)))

$\%$ end

$\%$ title('Peak "A-B" integrated vs Temp at increasing pwr');

$\%$ xlabel('Temp(k)');

$\%$ ylabel('Integrated intensity');

$\%$ hold off

$\% \%$ Integrate peak B-A vs power and temp \%\%\%\%\%\%\%\%\%\%\%\%\%\%\%\%\%\%\%\%\%\%\%

$\%$ figure(16)

$\%$ hold on

$\%$ for $m=1: 10$

$\%$ scatter(PowerAxis,log10(BA_Integrated $(m,:)))$

$\%$ plot(PowerAxis,log10(BA_Integrated $(m,:))$ )

$\%$ end

$\%$ title('Peak "B-A" integrated vs Power at increasing Temp');

$\%$ xlabel('Power (mW)');

$\%$ ylabel('Integrated intensity');

$\%$ hold off

$\%$

$\%$ figure(17)

$\%$ hold on

$\%$ for $m=1: 10$

\% scatter(TempAxis,log10(BA_Integrated(:,m)))

$\%$ plot(TempAxis,log10(BA_Integrated(:,m)))

$\%$ end

$\%$ title('Peak "B-A" integrated vs Temp at increasing pwr');

\% xlabel('Temp(k)');

$\%$ ylabel('Integrated intensity');

$\%$ hold off

$\% \%$ Light hole energy

$\%$ figure(18)

$\%$ hold on

$\%$ for $m=1: 10$

$\%$ scatter(PowerAxis,AEmEnergy $(m,:))$

$\%$ plot(PowerAxis,AEmEnergy $(m,:))$

$\%$ end

\% title('Ih Engy vs Power at increasing Temp');

$\%$ xlabel('Power (mW)');

$\%$ ylabel('Integrated intensity');

$\%$ hold off

$\% \%$

$\%$ figure(19) 
$\%$ hold on

$\%$ for $m=1: 10$

\% scatter(TempAxis,AEmEnergy $(:, m))$

$\%$ plot(TempAxis,AEmEnergy $(:, m))$

$\%$ end

\% title('Ih Engy vs Temp at increasing Power');

$\%$ xlabel('Temp(K)');

\% ylabel('Integrated intensity');

$\%$ hold off

\%\% Heavy hole energy

$\%$ figure(20)

$\%$ hold on

$\%$ for $m=1: 10$

$\%$

\% scatter(PowerAxis,BEmEnergy $(m,:))$

$\%$ plot(PowerAxis,BEmEnergy $(m,:))$

$\%$ end

\% title('hh Engy vs Power at increasing Temp');

\% xlabel('Power (mW)');

$\%$ ylabel('Integrated intensity');

$\%$ hold off

$\%$

\% figure(21)

$\%$ hold on

$\%$ for $m=1: 10$

$\%$

\% scatter(TempAxis,BEmEnergy(:,m))

$\%$ plot(TempAxis,BEmEnergy $(:, \mathrm{m}))$

$\%$ end

$\%$ title('hh Engy vs Temp at increasing Power');

\% xlabel('Temp(K)');

$\%$ ylabel('Integrated intensity');

$\%$ hold off

\% Integrate peaks A, B vs power at 6 K \%\%\%\%\%\%\%\%\%\%\%\%\%\%\%\%\%\%\%\%\%

\section{figure(22)}

hold on

for $m=1: 1$

scatter(PowerAxis,log10(A_Integrated $(m,:)))$

plot(PowerAxis,log10(A_Integrated(m,:)))

scatter(PowerAxis,log10(B_Integrated(m,:)))

plot(PowerAxis,log10(B_Integrated(m,:)),'-r')

end

title('Ih(blue) and hh(red) integrated vs Power at 6K');

xlabel('Power (mW)'); 


\section{ylabel('Integrated intensity'); hold off}

\%\% Integrated Peaks A, B at $40 \mathrm{~K}$

$\%$ figure(23)

$\%$ hold on

$\%$ for $m=10: 10$

\% scatter(PowerAxis,log10(A_Integrated $(m,:))$ )

$\%$ plot(PowerAxis,log10(A_Integrated $(m,:)))$

$\%$ scatter(PowerAxis,log10(B_Integrated $(m,:))$ )

$\%$ plot(PowerAxis,log10(B_Integrated(m,:)),'-r')

$\%$ end

$\%$ title('Ih(blue) and $\mathrm{HH}(\mathrm{red})$ integrated vs Power at $40 \mathrm{~K}$ ');

$\%$ xlabel('Power (mW)');

$\%$ ylabel('Integrated intensity');

$\%$ hold off

\% Integrate peaks A,B vs temp at low power \%\%\%\%\%\%\%\%\%\%\%\%\%\%\%\%\%\%\%\%\%\%\%

$\%$ figure(24)

$\%$ hold on

$\%$ for $m=1: 1$

$\%$ scatter(TempAxis,log10(A_Integrated(:,m)))

$\% \quad$ plot(TempAxis,log10(A_Integrated $(:, m)))$

$\%$ scatter(TempAxis,log10(B_Integrated(:,m)))

$\%$ plot(TempAxis,log10(B_Integrated(:,m)),'-r')

$\%$ end

$\%$ title('Ih(blue) and $\mathrm{hh}$ (red) integrated vs temp at .025mW pb');

$\%$ xlabel('Temp(K)');

$\%$ ylabel('Integrated intensity');

$\%$ hold off

$\%$

$\% \% \%$ Integrated Peaks A, B at vs temp at high power

$\%$ figure(25)

$\%$ hold on

$\%$ for $m=10: 10$

\% scatter(TempAxis,log10(A_Integrated(:,m)))

$\% \quad$ plot(TempAxis,log10(A_Integrated(:,m)))

$\%$ scatter(TempAxis,log10(B_Integrated(:,m)))

$\%$ plot(TempAxis,log10(B_Integrated(:,m)),'-r')

$\%$ end

$\%$ title('Ih(blue) and $\mathrm{HH}(\mathrm{red})$ integrated vs temp at $1.1 \mathrm{~mW} \mathrm{pb}$ ');

$\%$ xlabel('Temp(K)');

$\%$ ylabel('Integrated intensity');

$\%$ hold off 
$\% \quad \% \%$ Integrate peaks A-B and B-A vs power at low temp

\%\%\%\%\%\%\%\%\%\%\%\%\%\%\%\%\%\%\%\%\%\%\%

$\%$ figure(26)

$\%$ hold on

$\%$ for $m=1: 1$

\% scatter(PowerAxis,(AB_Integrated $(m,:)))$

$\% \quad \operatorname{plot}($ PowerAxis,(AB_Integrated $(m,:)))$

$\%$ scatter(PowerAxis,(BA_Integrated(m,: $))$ )

$\%$ plot(PowerAxis,(BA_Integrated(m,:)),'-r')

$\%$ end

$\%$ title('Ih-hh(blue) and hh-lh(red) integrated vs power at 6K');

$\%$ xlabel('Power (mW)');

$\%$ ylabel('Integrated intensity');

$\%$ hold off

$\%$

$\% \%$ Integrated Peaks A-B and B-A at vs power at high temp

$\%$ figure(27)

$\%$ hold on

$\%$ for $m=10: 10$

$\%$ scatter(PowerAxis,(AB_Integrated $(m,:)))$

$\%$ plot(PowerAxis,(AB_Integrated $(m,:)))$

$\%$ scatter(PowerAxis,(BA_Integrated(m,:)))

$\% \operatorname{plot}($ PowerAxis,(BA_Integrated(m,:)),'-r')

$\%$ end

$\%$ title('Ih-hh(blue) and $\mathrm{HH}-\mathrm{Ih}($ red) integrated vs power at $40 \mathrm{K'}$ );

$\%$ xlabel('Power (mW)');

$\%$ ylabel('Integrated intensity');

$\%$ hold off

$\% \quad \% \%$ Integrate peaks A-B and B-A vs twmp at low power

\%\%\%\%\%\%\%\%\%\%\%\%\%\%\%\%\%\%\%\%\%\%\%

$\%$ figure(26)

$\%$ hold on

$\%$ for $m=1: 1$

$\%$ scatter(TempAxis,(AB_Integrated(:,m)))

$\%$ plot(TempAxis,(AB_Integrated $(:, m)))$

$\%$ scatter(TempAxis,(BA_Integrated(:,m)))

$\%$ plot(TempAxis,(BA_Integrated(:,m)),'-r')

$\%$ end

$\%$ title('Ih-hh(blue) and hh-lh(red) integrated vs Temp at .025mw PB');

$\%$ xlabel('Temp (K)');

\% ylabel('Integrated intensity');

$\%$ hold off

$\%$

$\% \%$ Integrated Peaks A-B and B-A at vs power at high temp 


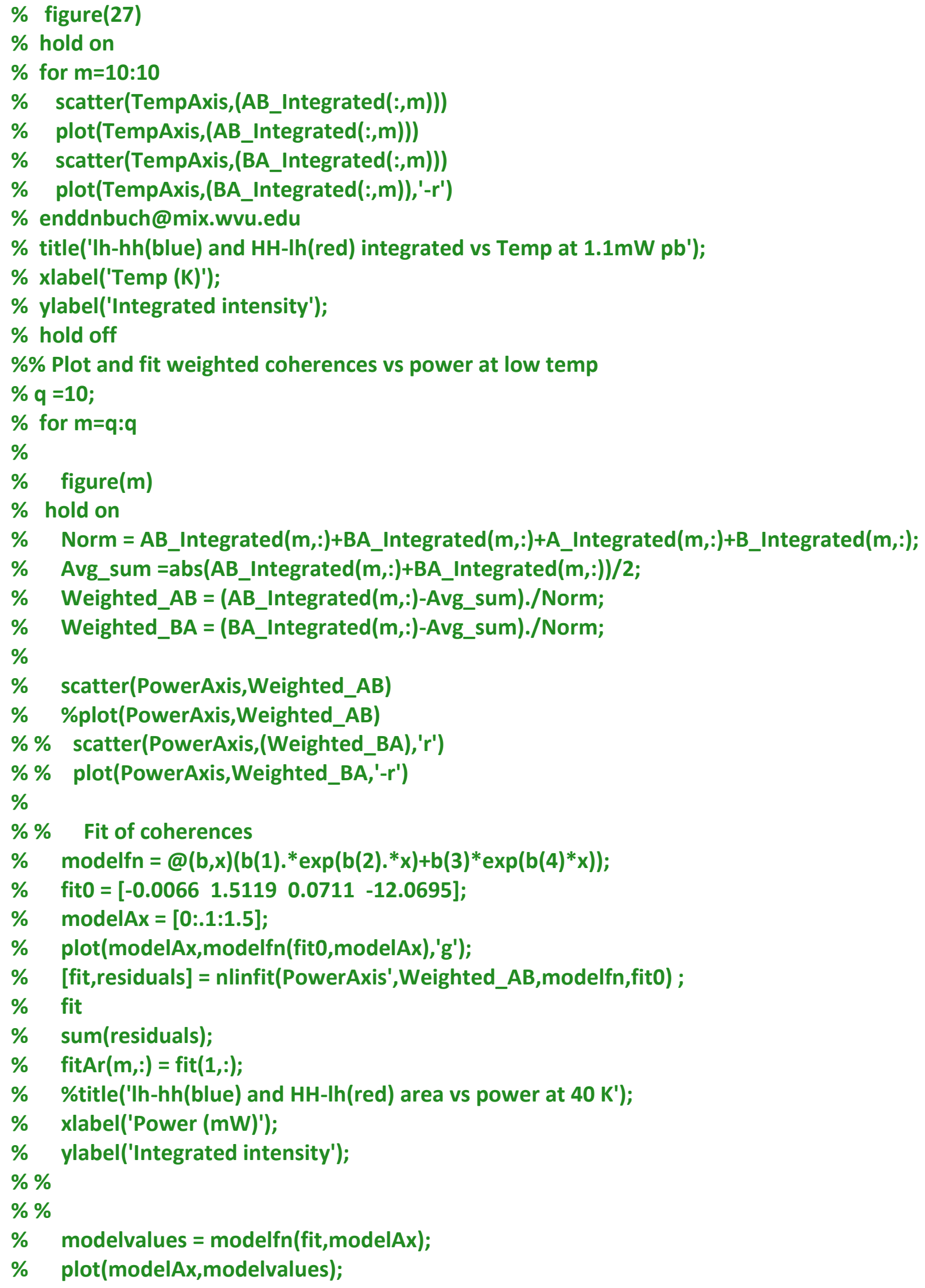




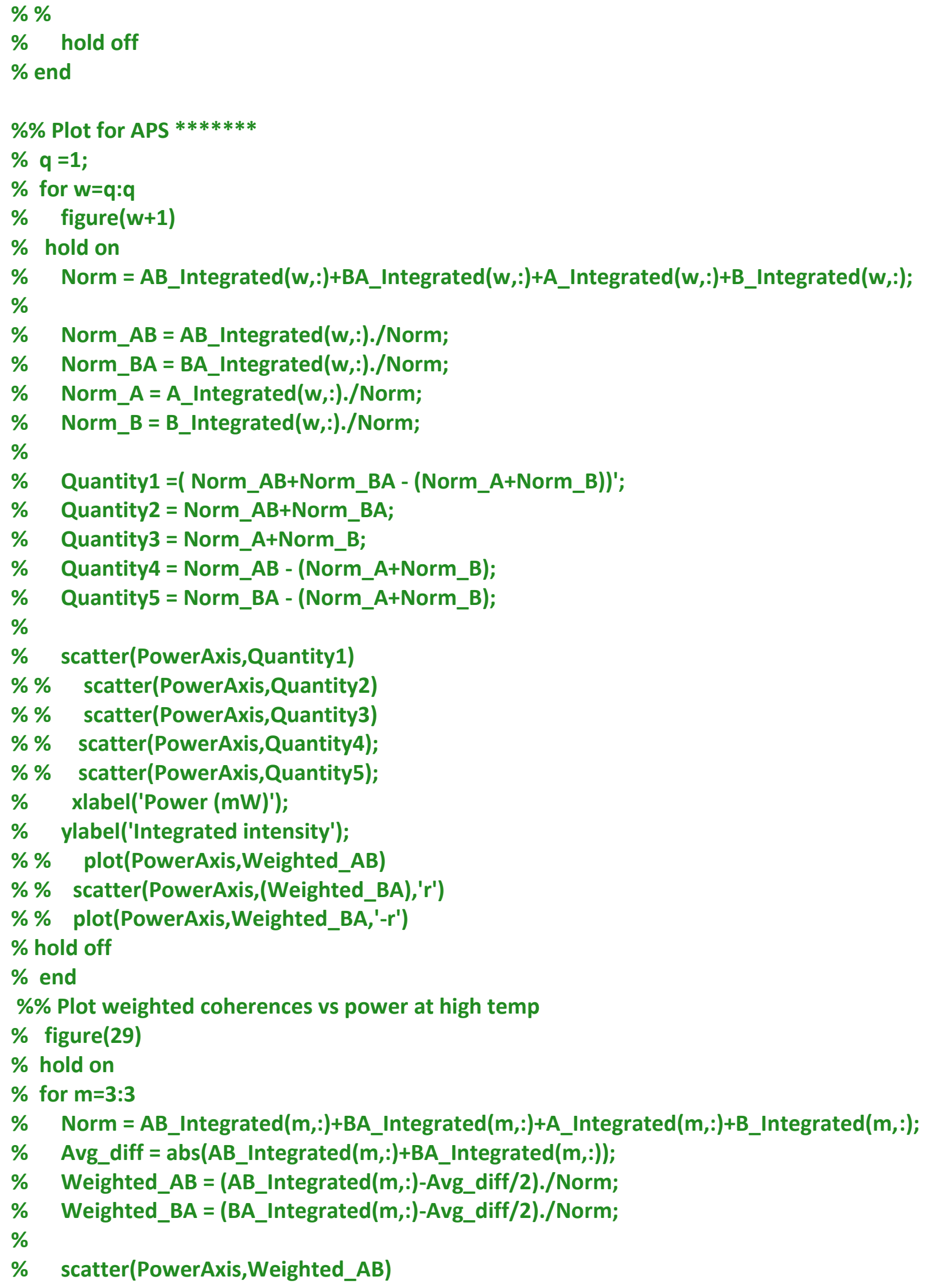


$\%$ scatter(PowerAxis,(Weighted_BA))

$\%$ plot(PowerAxis,Weighted_BA,'-r')

$\%$ end

$\%$ title('Ih-hh(blue) and $\mathrm{HH}-\mathrm{Ih}($ red) area vs power at increasig $10 \mathrm{K'}$ );

$\%$ xlabel('Power $(\mathrm{mW})$ ');

$\%$ ylabel('Integrated intensity');

$\%$ errorbar([.15 .2],[Weighted_AB(4) Weighted_AB(5)],[Weighted_AB(4)-.09219

Weighted_AB(5)-.08363]);

$\%$ errorbar([.15 .2],[-Weighted_AB(4) -Weighted_AB(5)],[Weighted_AB(4)-.09219

Weighted_AB(5)-.08363],'r');

$\%$ hold off

$\% \%$ Plot weighted coherences vs temp at low power

$\%$ figure(30)

$\%$ hold on

$\%$ for $m=1: 1$

$\% \quad$ Norm $=A B \_I n t e g r a t e d(:, m)+B A \_I n t e g r a t e d(:, m)+A \_I n t e g r a t e d(:, m)+B \_I n t e g r a t e d(:, m)$;

$\% \quad$ Avg_diff =abs(AB_Integrated(:,m)+BA_Integrated $(:, m))$;

$\% \quad$ Weighted_AB $=\left(A B \_I n t e g r a t e d(:, m)-A v g \_d i f f / 2\right) . / N o r m ;$

$\%$ Weighted_BA $=\left(\right.$ BA_Integrated $\left.(:, m)-A v g \_d i f f / 2\right) . /$ Norm;

$\%$

$\% \quad$ scatter(TempAxis,Weighted_AB)

$\%$ plot(TempAxis,Weighted_AB)

$\%$ scatter(TempAxis,(Weighted_BA))

$\%$ plot(TempAxis,Weighted_BA,'-r')

$\%$ end

$\%$ title('Ih-hh(blue) and $\mathrm{HH}-\mathrm{Ih}(\mathrm{red})$ area vs twmp at $.025 \mathrm{~mW}$ pb');

$\%$ xlabel('Temp (K)');

$\%$ ylabel('Integrated intensity');

$\%$ hold off

$\% \%$ Plot weighted coherences vs temp at high power

$\%$ figure(31)

$\%$ hold on

$\%$ for $m=10: 10$

$\% \quad$ Norm $=$ AB_Integrated $(:, m)+B A \_I n t e g r a t e d(:, m)+A \_I n t e g r a t e d(:, m)+B \_I n t e g r a t e d(:, m)$;

$\% \quad$ Avg_diff $=$ abs(AB_Integrated $\left.(:, m)+B A \_I n t e g r a t e d(:, m)\right)$;

$\% \quad$ Weighted_AB $=\left(A B \_I n t e g r a t e d(:, m)-A v g \_d i f f / 2\right) . /$ Norm;

$\%$ Weighted_BA $=($ BA_Integrated(:,m)-Avg_diff/2)./Norm;

$\%$

$\%$ scatter(TempAxis,Weighted_AB)

$\%$ plot(TempAxis,Weighted_AB)

$\%$ scatter(TempAxis,(Weighted_BA))

$\%$ plot(TempAxis,Weighted_BA,'-r')

$\%$ end 
$\%$ title('Ih-hh(blue) and $\mathrm{HH}-\mathrm{Ih}($ red) area vs temp at $1.1 \mathrm{~mW}$ pb');

\% xlabel('Temp (K)');

$\%$ ylabel('Integrated intensity');

$\%$ hold off

$\%$

$\% \% \%$ Heavy and light energy overly vs power

$\%$ figure(31)

$\%$ hold on

$\%$ for $m=1: 1$

$\%$ scatter(PowerAxis,AEmEnergy $(m,:))$

$\%$ plot(PowerAxis,AEmEnergy $(m,:))$

\% scatter(PowerAxis,BEmEnergy(m,:),'r')

$\%$ plot(PowerAxis,BEmEnergy(m,:),'r')

$\% \quad y \lim ([1502$ 1511] )

$\%$ end

$\%$ title('Ih(blue) hh(red) Engy vs Power at 6k');

$\%$ xlabel('Power (mW)');

\% ylabel('Emission energy (meV)');

$\%$ hold off

$\% \% \%$ Heavy and light energy overly vs temp

$\%$ figure(32)

$\%$ hold on

$\%$ for $m=1: 1$

\% scatter(TempAxis,AEmEnergy(:,m))

$\%$ plot(TempAxis,AEmEnergy(:,m))

\% scatter(TempAxis,BEmEnergy(:,m),'r')

$\%$ plot(TempAxis,BEmEnergy(:,m),'r')

$\%$ end

$\%$ title('lh(blue) hh(red) Engy vs Temp at .025 mW pb');

$\%$ xlabel('Temp (K)')

$\%$ ylabel('Emission energy (meV)');

$\%$ hold off 